ATMOSPHERIC INTERACTIONS WITH

GULF STREAM RINGS

by

William K. Dewar

B.S. the Ohio State University

(1977)

S.M. the Massachusetts Institute of Technology

(1980)

\title{
S.M. the Massachusetts Institute of Technology
}

\begin{abstract}
SUBMITTED IN PARTIAL FULFILLMENT
OF THE REQUIREMENTS FOR THE DEGREE OF

DOCTOR OF PHILOSOPHY
\end{abstract}

\author{
at the \\ MASSACHUSETTS INSTITUTE OF TECHNOLOGY \\ and the \\ WOODS HOLE OCEANOGRAPHIC INSTITUTION \\ October 1982
}
Signature of Author
Department of Meteorology and Physical Oceanography, Massachusetts Institute of Technology and the Joint Program in Oceanography, Massachusetts Institute of Technology/Woods Hole Oceanographic Institution, October, 1982.

Certified by

Thesis Supervisor

Accepted by

Chairman, Joint Committee for Physical Oceanography, Massachusetts Institute of Technology/Woods Hole Oceanographic Institution. 
by

William K. Dewar

Submitted to the MIT-WHOI Joint Program

in Physical Oceanography on 8 October, 1982

in partial fulfillment of the requirements for the degree of Doctor of Philosophy

\section{ABSTRACT}

Four different problems concerning Gulf Stream Rings are considered. The first deals with the particle trajectories of, and advection-diffusion by, a dynamic model of a Ring. It is found that the streaklines computed from the assumptions that the Ring is a steadily propagating and permanent form structure accurately describe its Lagrangian trajectories. The dispersion field of the Ring produces east-west asymmetries in the streaklines, not contained in earlier kinematic studies, which are consistent with observed surface patterns. In the second problem, we compute the core mixed layer evolution of both warm and cold Rings, and compare them to the background SST, in an effort to explain observed SST cycles of Rings. We demonstrate that warm Rings retain their anomalous surface identity, while cold Rings do not, because of differences in both the local atmospheric states of the Sargasso and the Slope and the typical mixed layer structures appropriate to each. The third and fourth problems concern the forced evolution of Gulf Stream Rings as effected by atmospheric interactions. First, we compute the forced spin down of a Gulf Stream Ring. The variations in surface stress across the Ring necessary to spin it down are caused by the variations in relative air-sea velocity, of which the stress is a quadratric function. From numerical simulations, we find the forced decay rates are comparable to those inferred from Ring observations. In the final problem, it is suggested that a substantial fraction of meridional Ring migration is a forced response, caused by Ring SST and the temperature dependence of stress. The warm central waters of anticyclonic Rings are regions of enhanced stress, producing upwelling to the north, and downwelling to the south, which shifts the Ring to the south. A similar, southward shift is computed for cyclonic Rings with cold centers, which tends to reconcile their numerically computed propagation with observations. 
TABLE OF CONTENTS

Title Page $\ldots \ldots \ldots \ldots \ldots \ldots \ldots \ldots \ldots \ldots \ldots \ldots \ldots \ldots \ldots \ldots$

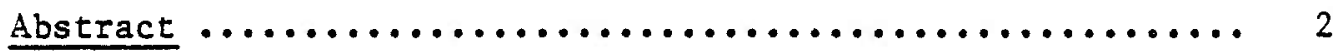

Table of Contents $\ldots \ldots \ldots \ldots \ldots \ldots \ldots \ldots \ldots \ldots \ldots \ldots \ldots$

Chapter I. Introduction $\ldots \ldots \ldots \ldots \ldots \ldots \ldots \ldots \ldots \ldots \ldots$. 7

Ring Observations and Description ........... 8

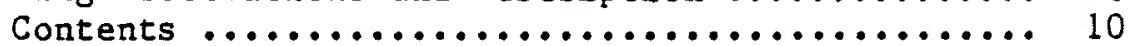

Chapter II. Preliminaries $\ldots \ldots \ldots \ldots \ldots \ldots \ldots \ldots \ldots \ldots \ldots$

a. Introduction .......................... 15

b. The Quasi-Geostrophic Horizontal Structure

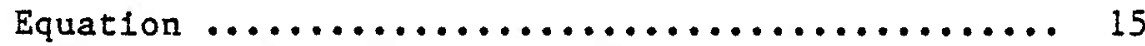

The Equivalent Barotropic Equation ........... 25

A Discussion of Baroclintc Instability ........ 26

c. Advection-Diffusion of a Passive Scalar ......... 27

d. The Mixed Layer ........................ 28

Conservation of Mass and Thermodynamic Energy ... 29

Momentum Equations ..................... 31

Quasi-Geostrophic Scaling ................ 32

Ekman Pumping ......................... 34

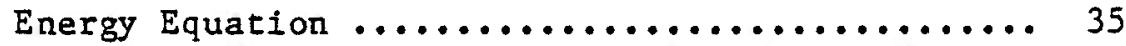

Wind Wave Breaking and Penetrative Convection ... 38

The Froude Number and Its Value ............ 39

e. Numerical Techniques ..................... 40

Chapter III. Particle Trajectories in Numerical Gulf

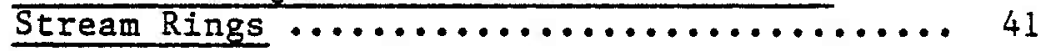

a. Introduction $\ldots \ldots \ldots \ldots \ldots \ldots \ldots \ldots \ldots \ldots \ldots \ldots \ldots, 41$

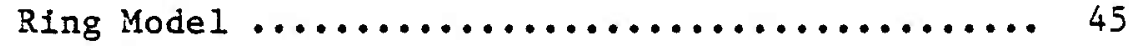

b. Kinematic Models ....................... 46

Tracer Diffusion in Kinematic Models .......... 49

Tracer Homogenization on Closed Streamlines ..... 49

c. Advection and Diffusion in a Dynamic Ring Model .. 62

Dynamic 'Streaklines' ....................... 64

The Importance of the Dispersion Fleld ........ 70

Advection-Diffusion Using Dynamical Advection

Flelds ............................. 72

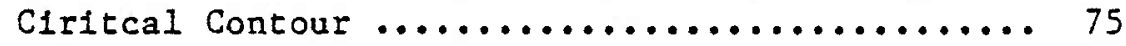

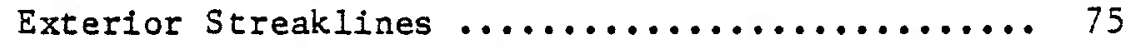

d. Potential Vorticity Considerations ............ 80

Ring Exterior ........................ 83

e. Implications .......................... 84

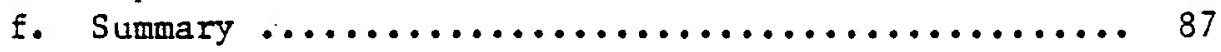


Chapter IV. An Annual Mixed Layer Model with Application to Gulf Stream Rings .......... 90

a. Introduction $\ldots \ldots \ldots \ldots \ldots \ldots \ldots \ldots \ldots \ldots \ldots \ldots \ldots$

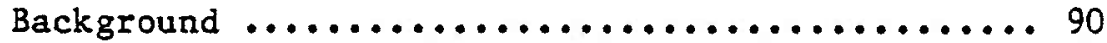

b. An Annual Mixed Layer Model ................. 95

c. Limit Cycle Calculations ...................101

'Typical' Mixed Layers ......................103

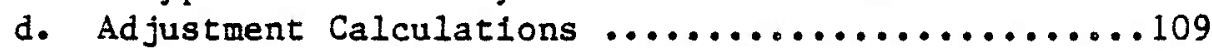

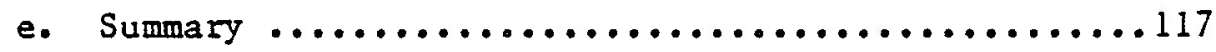

Appendix A.IV. A Bulk Mixed Layer Model $\ldots \ldots \ldots \ldots \ldots 121$

a. The Equations and the Forcing Functions ........121

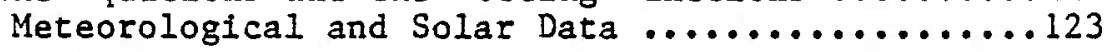

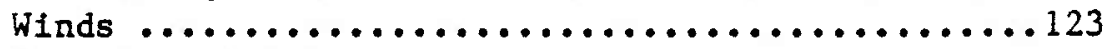

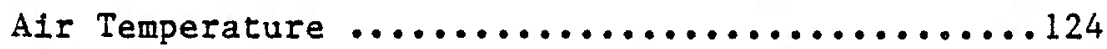

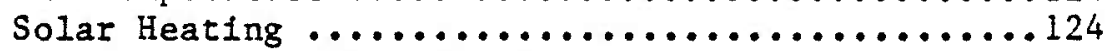

b. Initial Experiments with the Thompson Model .....127

Appendix B.IV. The Sensitivity of Mixed Layer Development to Buoyancy Flux ...........131

The Reformation of the Thermocline ...........131

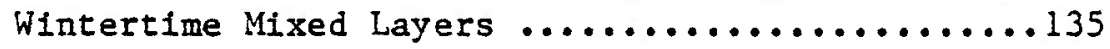

Appendix C.IV. Verification of the Annual Mixed Layer

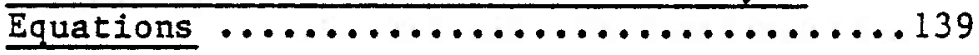

a. Choice of $\mathrm{h}$

b. Validation $0 . \ldots \ldots \ldots \ldots \ldots \ldots \ldots \ldots \ldots \ldots \ldots \ldots 139$ 
Chapter V. The Wind Forced Spin Down of Gulf Stream

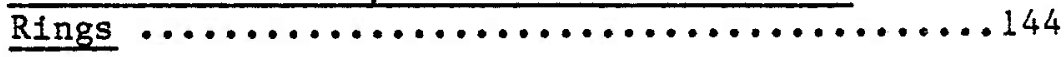

a. Introduction ............................... 144

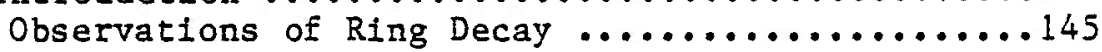

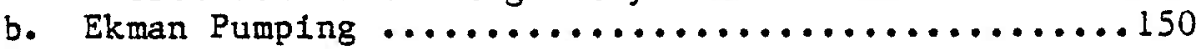

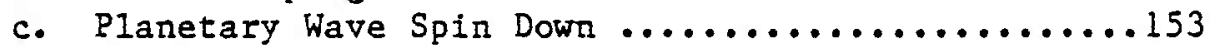

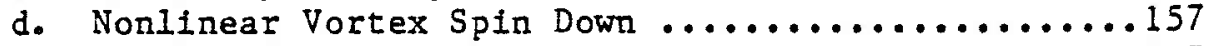

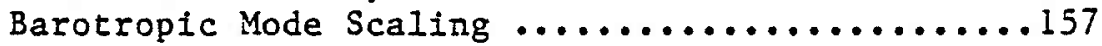

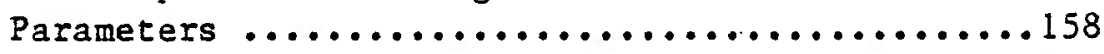

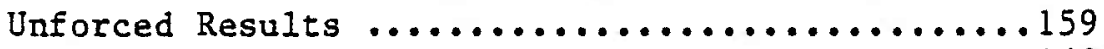

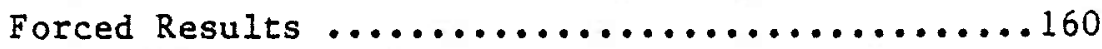

The Relative Importance of Forcing ............167

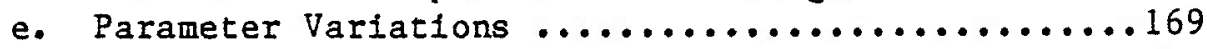

f. The Spin Down Mechanism .....................171

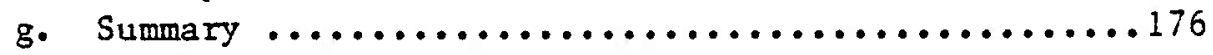

Appendix A.V. Wind Stress in the Presence of Surface

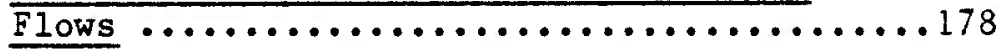

Chapter VI. Southward Ring Propagation as a Consequence of Surface Temperature Anomalies ............184

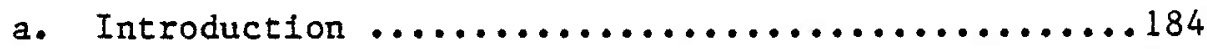

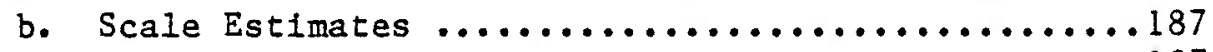

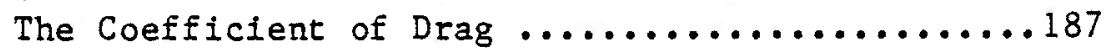

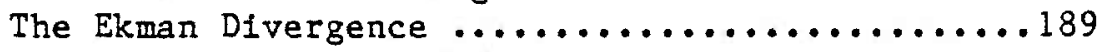

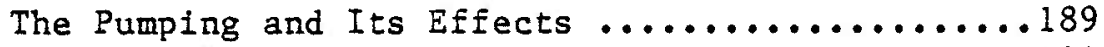

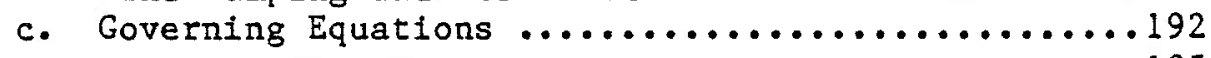

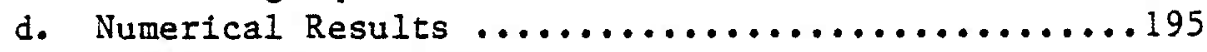

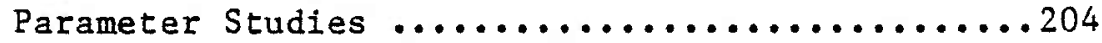

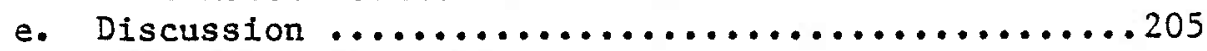

Integral Constraints ...................206

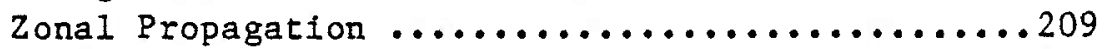

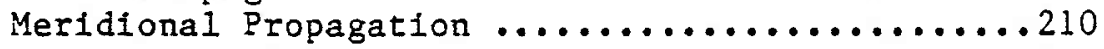

f. Potential Vorticity Budgets ................. 11

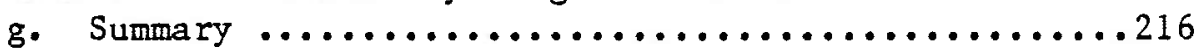




\section{Page -6-}

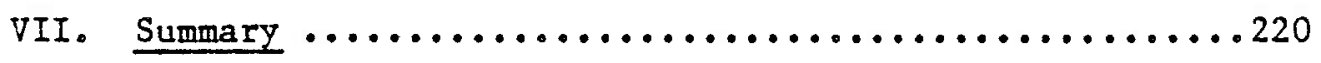

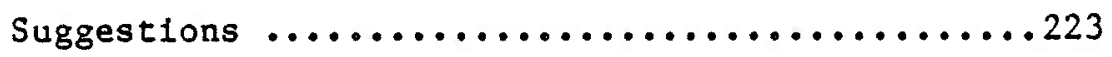

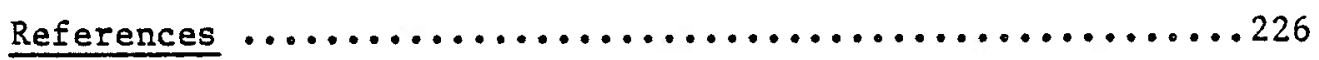

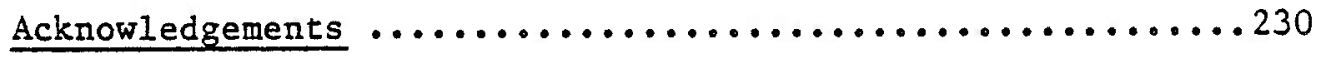


CHAPTER I. INTRODUCTION

Gulf Stream Rings are intense vortices shed by the Gulf Stream, characterized by velocities up to $150 \mathrm{~cm} / \mathrm{sec}$ and diameters of about 100 km. They are commonly found in the Slope Water and the Sargasso, and as such constitute the most energetic time dependent phenomena in either reglon. Rings transport water between the Slope Water and the Sargasso, which has led scientists to suggest that they are a dominant component in the heat and energy budgets of both regions. For example, the potential vorticity flux to the Sargasso caused by Rings has been estimated at $1 \mathrm{~m}^{2} / \mathrm{sec}^{2}$, which is the same magnitude as that due to the atmosphere (the Ring Group, 1981). Similar statements apply to Ringinduced salt and heat flux. In addition, the powerful velocities of a Ring can strain existing tracer gradients, enhancing their diffusive transport. Thus, it is very likely that Rings are important to the large scale picture of the oceans. Whether Rings produce effects as large as these estimates, or alter their environment in a significant way, is the focus of current observational and theoretical effort (Richardson, 1980).

The areas essential to addressing these questions, in which our knowledge is incomplete, include Ring decay, propagation, and transport. Recent modeling efforts (McWilliams and Flierl, 1979; Mied and Lindemann, 1979; Ikeda, 1981; Nof, 1980; Flierl, 1982) have centered on the evolution of freely evolving structures imbedded in a resting body of water. Although several of these studies have mentioned the 
potential importance of mean state advection and external forcing, there have been only a few attempts at including shear (Flierl, 1979) and wind stress (Stern, 1965) in eddy calculations. In the present thesis, we will consider how atmospheric forcing affects the evolution of Rings, and demonstrate that several of their oceanographically important properties are significantly influenced by air-sea exchange. In particular, we shall see that Ring decay and propagation are affected by wind forcing, and that the evolution of Ring surface waters is sensitive to diabatic heating. Also, because many of the processes involved in these problems are more naturally discussed in a Lagrangian frame, and because of the importance of Ring advection on their surroundings, we have computed the particle trajectories of a Gulf Stream Ring.

Ring Observations and Description-

Rings are distinguished from the mesoscale variability of the North Atlantic primarily in two ways. First, they undergo a unique formation process: Gulf Stream meanders grow to finite amplitude, close, and subsequently separate from the current. Second, Rings carry with them a sizeable volume of distinctive water. Ring production has been observed to occur on both sides of the stream, producing vortices of positive (cyclonic) rotation to the south and negative (anticyclonic) rotation to the north. Similar structures are found in the vicinity of most major current systems (Hamon, 1960; Nilsson, Andrews, and Scully-Power, 1977; Kawai, 1979), although presently, the literature is most complete for the North Atlantic. 


$$
\text { Page -9- }
$$

The first well documented long term observation of a single Ring (cyclonic) is due to Fuglister (1977), who was able to track the same Ring for six months. To date, several Rings have been tracked (RIchardson, 1980) and many of their common physical properties catalogued. Rings persist as recognizable coherent structures, literally as closed loops of flow, for years at a time (Parker, 1971). They translate toward the west-southwest at speeds of about $5 \mathrm{~cm} / \mathrm{sec}$ but can exhibit rapid eastward motion when interacting with the Gulf Stream. As many as 10 cyclonic and 6 anti-cyclonic Rings have been observed to coexist. They are formed at a rate of about 7 Rings per year and are frequently removed from the general circulation by reabsorption into the Gulf Stream (Richardson, 1980). For a more complete descriptive review of Rings, see Lai and Richardson (1977).

During formation, large pieces of water are trapped within the closing meanders which results in Rings having a peculiar water mass composition. For example, a cyclonic Ring in the Sargasso Sea will have an interfor consisting of Slope Water. The strong temperature contrasts between the Slope water and the Sargasso have led to the now standard labels of 'cold core Ring' for those found in the Sargasso, and 'warm core Ring' for those in the Slope. The formation process also suggests some other terms which will be used in the present manuscript. The region into which the newly formed Ring moves will be referred to as the 'host region', and the area from which the core waters originated will be called the 'parent region'. As an example, the Sargasso Sea is the host region of a cold core Ring and the Slope Water the parent region. 


\section{Contents-}

The following brief summaries of each chapter will serve as a guide to the new results in this thesis.

\section{Chapter III}

Flierl (1981) computed the particle trajectorles of a steadilypropagating, axisymmetric pressure pattern with closed streamlines. By applying this model to Rings, he was able to make many useful statements with regards to the structure of particle tracks, trapped zone size, and averaged Lagrangian velocities. This study was purely kinematic, and employed a velocity field which turned out to be dynamically inconsistent, although it did come from an analysis of Ring data (OIson, 1980). In Chapter III, we conduct a Lagrangian analysis of a dynamically evolving Ring, the equivalent barotropic Ring model originally proposed by McWilliams and Flierl (1979). Comparisons between the dynamical model streaklines and those of the kinematic study are made which point out where the earlier calculations adequately describe particle motion and where improvements are needed. The particle trajectories of the dynamic Ring are investigated in terms of potential vorticity, and the importance of the dispersion field is discussed. We conclude Chapter III with an example of Ring interaction with tracer boundaries, performed with a view towards modeling Ring-thermal front interactions. 


\section{Chapter IV}

In satellite infra-red images, Rings generally show up as we11defined pools of anomalously warm or cold water. Thus, one of the first cycles to be observed by the remote sensing program was that of the annual Ring sea surface temperature. It is now documented that cold core Ring surface waters do not survive beyond their first summer as an identifiable cold pool (the Ring Group, 1981); however, warm core Rings, with the possible exception of summertime, remain visible throughout their lifetime in satellite infra-red 1mages. From XBT data, we find evidences of strong air-sea exchange and deep mixed layers in warm core Rings, and a curious lack of unusual surface water development in cold core Rings. In Chapter IV, we consider mixed layer evolution on the annual time scale, with particular emphasis on explaining the features of Ring SST cycles. Using a one dimensional model, we compare the forced response of the core surface layer of a Ring to that of its flank, demonstrating what aspects of the observed surface temperature field can be attributed to local air-sea exchange. This view differs from the pervading idea that it is the Ring dynamics which are responsible for the sea surface temperature (SST) behavior. We also apply the results of this study to the interpretation of satellite infra-red images. The model, within the restrictions of one-dimensionality, suggests how to objectively interpret SST anomalies. 
Rings persist for years at a time (Lai and Richardson, 1977), although they do experience a recognizable aging process (Richardson, Maillard, and Sanford, 1981). Various estimates of decay rates have been made using observed subsidence of isotherms (Parker, 1971) or loss of potential energy (Cheney and Richardson, 1976) and suggest lifetimes of roughly two-three years. One of the classic problems of Ring evolution concerns the method by which Rings lose their energy. It was concluded by McWilliams and Flierl (1979), as well as by Meid and Lindemann (1979), that vortex decay in their numerical experiments was strongly influenced by viscosity and that the usually dominant dispersive decay mechanism was in large prevented by the strength of the flow. The lack of a well-founded closure theory prevented them from making any definitive statements with regards to decay beyond a recognition of the importance of the weak non-conservative processes. In Chapter $V$, we investigate the possibility that Ring spin down is a result of Ekman divergence driven by local variations of momentum transfer at the sea surface. The bulk formula for stress is a quadratic function of the relative air-sea velocity; therefore, the presence of intense surface velocities can induce local, non-negligible, gradients of stress. The dissipative nature of the forcing, similar to bottom friction, emerges from the calculation of the Ekman pumping; one of the more useful results is the analytical expression for what corresponds to the coefficient of viscosity multiplying the frictional operator. A serles of numerical experiments, including the pumping, are performed and the results compared to oceanic observations of Ring decay. 


\section{Chapter VI}

In Chapter VI, we consider the effects of the local variations in stress on a Ring caused by its surface temperature field. The dependence of the bulk aerodynamic coefficient of drag on the temperature difference between the air and water has been documented by Deardorff (1968), and produces $0(50 \%)$ variations in stress for temperature contrasts on the order of a few degrees Centigrade. Ring surface temperature anomalies are such that both Ekman suction and pumping are produced, forcing the Ring to the south. We present numerical experiments, which include surface temperature anomalies, to demonstrate this effect and discuss the dynamical balances which account for the meridional propagation. McWilliams and Flierl (1979) point out that according to quasi-geostrophic dynamics, freely evolving cyclonic vortices (cold core Rings) move northward; a result which is counter to most observations. One of the interesting results of Chapter VI is that both warm and cold core Rings are compelled towards southward motion, which brings the predicted propagation of cold Rings more into accord with observations.

\section{Chapters II and VII}

The relevant equations are derived and catalogued in Chapter II. First, we discuss a two degree of freedom quasigeostrophic model in both layered and continuously stratified modal forms (Flierl, 1978) and review the validity of the equivalent barotropic equation. This is followed by derivations of the advection-diffusion equation and the 
basic mixed layer equations. Chapter VII contains a summary together with a discussion of future research topics suggested by this work. 


\section{II.a Introduction-}

The purpose of this chapter is to derive and catalog the fundamental equations which we will frequently use. Necessarily, some of the content of the next few pages will not be new; for example, we review the derivation of the quasi-geostrophic modal equations as originally formulated by Filerl (1978). On the other hand, a rather original derivation of Ekman pumping as the upper boundary condition on the mesoscale will be presented in the section dealing with the mixed layer. In all sections, we will point out the relevant physics contained within each equation. The reader already familiar with general areas of quasi-geostrophy, advection-diffusion, and mixed layers can skip directly to Chapter III. It should be noted however that the notation employed in this chapter will become standard, thus reference to the tables and sections contained herein should resolve any questions with respect to symbol definition.

\section{II. b The Quasi-Geostrophic Horizontal Structure Equation-}

The basic equation describing the dynamics of the mesoscale is the quas1-geostrophic psuedo-potential vorticity conservation equation. In dimensional form, this equation is:

$$
\frac{d}{d t}\left[o^{2} p+\frac{j}{j z}\left(\frac{f_{o}^{2}}{N^{2}} \frac{j}{j z} P\right)+\beta f_{0} y\right]=0, \quad \text { Eq. II.L }
$$


Table II. 1 Symbols and Definitions

\begin{tabular}{|c|c|c|}
\hline & mbol & Meaning \\
\hline \multicolumn{3}{|c|}{ Environmental Symbols } \\
\hline 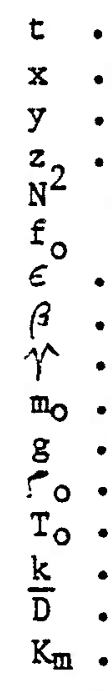 & 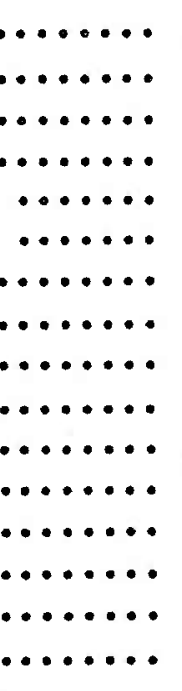 & $\begin{array}{l}\text { Time } \\
\text { Zonal coordinate } \\
\text { Meridional coordinate } \\
\text { Depth } \\
\text { Buoyancy frequency } \\
\text { Coriolis parameter } \\
\text { Mixed layer dissipation } \\
\text { Meridional Gradient of f } \\
\text { Coefficient of seawater thermal expansion } \\
\text { Energy equation coefficient } \\
\text { Gravity } \\
\text { Reference density } \\
\text { Reference temperature } \\
\text { Unit vertical vector } \\
\text { Passive scalar Decay rate } \\
\text { Passive scalar coefficient of diffusion }\end{array}$ \\
\hline \multicolumn{3}{|c|}{ Scales and Nondimensional Parameters } \\
\hline 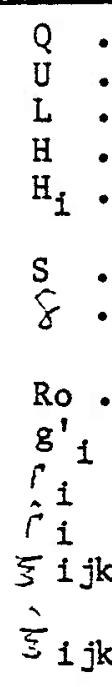 & 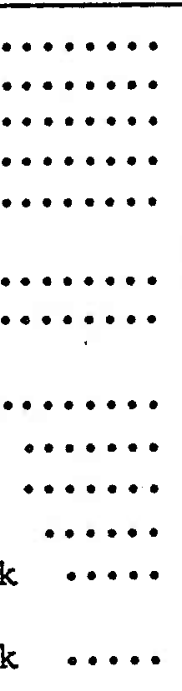 & $\begin{array}{l}\text { Steepness }=\mathrm{U} /\left(\mathrm{RL}^{2}\right) \\
\text { Velocity scale } \\
\text { Horizontal length scale } \\
\text { Depth scale } \\
\text { Average layer thickness } \\
\text { Burger number }\left(=(\mathrm{NH}) 2 /\left(\mathrm{f}_{\mathrm{OL}}\right)^{2}\right) \\
\text { Depth ratio }\left(=\mathrm{H}_{1} / \mathrm{H}_{2}\right) \\
\text { Rossby number }\left(=\mathrm{U} /\left(\mathrm{f}_{\mathrm{OL}}\right)\right) \\
\text { Density step } \\
\text { Continuous separation constant } \\
\text { Layered separation constant } \\
\text { Continuous modal interaction parameter } \\
\text { Layered modal interaction parameter }\end{array}$ \\
\hline \multicolumn{3}{|c|}{ Variables } \\
\hline $\begin{array}{l}\mathrm{u} \\
\mathrm{v} \\
\mathrm{w} \\
\mathrm{u}_{i} \\
\mathrm{v}_{\mathrm{i}} \\
\mathrm{P} \\
\mathrm{P}_{i} \\
\mathrm{~F}_{i}\end{array}$ & 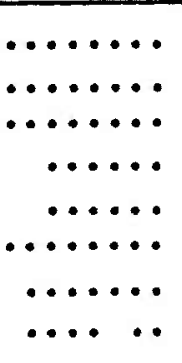 & $\begin{array}{l}\text { Zonal velocity } \\
\text { Meridional velocity } \\
\text { Vertical velocity } \\
\text { Intermediate Layer zonal Velocity } \\
\text { Intermediate Layer meridional velocity } \\
\text { Pressure } \\
\text { Layer pressure } \\
\text { Continuous modal structure }\end{array}$ \\
\hline
\end{tabular}




$$
\text { Page -17- }
$$

Table II. 1 Symbols and Definitions (continued)

\begin{tabular}{|c|c|}
\hline Symbol & Meaning \\
\hline \multicolumn{2}{|c|}{ Variables (continued) } \\
\hline 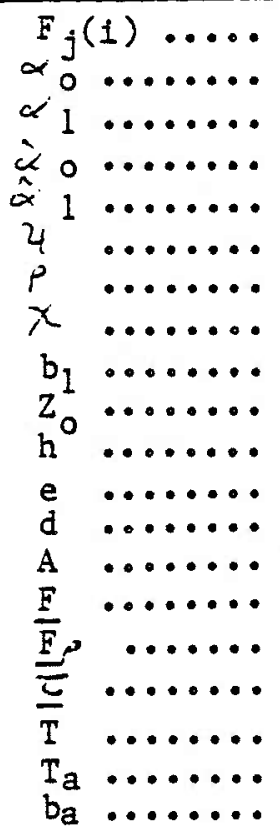 & $\begin{array}{l}\text { Layered modal structure } \\
\text { Continuous barotropic horizontal structure } \\
\text { Continuous baroclinic horizontal structure } \\
\text { Layered barotropic horizontal structure } \\
\text { Layered baroclinic horizontal structure } \\
\text { Rescaled barotropic horizontal structure } \\
\text { Density } \\
\text { Rescaled baroclinic horizontal structure } \\
\text { Intermediate layer buoyancy } \\
\text { Level depth under intermediate layer } \\
\text { Mixed layer depth } \\
\text { Entrainment rate } \\
\text { Isopycnal displacement } \\
\text { Passive scalar concentration function } \\
\text { Internal wave radiation stress } \\
\text { Turbulent density flux } \\
\text { Wind stress } \\
\text { Temperature of seawater } \\
\text { Temperature of air } \\
\text { buoyancy of air }\end{array}$ \\
\hline \multicolumn{2}{|r|}{ Mathematical Operators and Symbols } \\
\hline Symbol & Meaning \\
\hline $\begin{array}{l}\nabla_{J(A, B)} \ldots \\
\operatorname{curl(A)} \ldots \\
\delta_{i j} \ldots \\
\end{array}$ & $\begin{array}{l}\partial^{2} /(\partial x)^{2}+\partial^{2} /(\partial y)^{2} \\
A_{x^{B}}-B_{x} A_{y} \\
\left(A_{y}\right)_{x}-\left(A_{x}\right)_{y} \\
\text { Kronecker delta }(=0 \text { if } i=j, 1 \text { if } i=j)\end{array}$ \\
\hline
\end{tabular}


where $d / d t$, the substantial time derivative, is defined by:

$$
\frac{d}{d t}=\frac{\partial}{\partial t}+\frac{1}{f_{0}} J(P, .)=\frac{\partial}{\partial t}+\frac{1}{f_{0}}\left(\frac{\partial}{d x} P \frac{\partial}{\partial y}-\frac{\partial}{\partial y} P \frac{\partial}{\partial x}\right) . \quad E q \cdot I I_{0} 2
$$

Eqs. II.1 and 2 describe exchanges between relative vorticity, vortex stretching, and planetary vorticlty, along the horizontal projection of a particle trajectory, such that those exchanges conserve potential vorticity. For a complete derivation of this equation, see Pedlosky (1979). The proper vertical boundary conditions for Eq. II.l are on the vertical velocity of the flow:

$$
\mathrm{d} / \mathrm{dt}\left(\mathrm{P}_{z}\right)=-\mathrm{N}^{2} \mathrm{w}
$$

at $z=0$ and $-H$. We will generally assume a flat bottom ( $w=0$ at $z=-H$ ), but allow for a surface divergence. For horizontal boundary conditions, we shall assume for numerical purposes a doubly periodic domain:

$$
P\left(x+L_{x}, y+L_{y}\right)=P(x, y) \text {. }
$$

Non-dimensionalizing $x$ and $y$ by $L, t$ by $\left(\beta_{L}\right)^{-1}, u$ and $v$ by $U$, $P$ by $f_{O U L}, w$ by $U 2 H /\left(f_{O L} 2\right)$, and $z$ by $H$ (see Table II. 1 ) returns:

$$
\left[\frac{d}{d t}+Q J(P, .)\right]\left[v^{2} P+\frac{j}{j z} \frac{1}{S} \frac{\partial}{J z} P\right]+P_{x}=0, \quad \text { Eq. II.3 }
$$

where $Q=U /\left(\beta_{L} 2\right)$, and $S$ is the Burger number, defined by $S=$ $(\mathrm{NH})^{2} /\left(\mathrm{f}_{\mathrm{OL}}\right)^{2}$. The vertical boundary conditions become:

$$
\left[\frac{\partial}{\partial t}+Q J(P, .)\right] \frac{\partial}{\partial z} P=-S w \quad E q \cdot I I .4
$$

at $z=0$ and -1 . 
If the upper and lower boundary conditions are homogeneous, the linear form of Eq. II.l becomes mathematically separable, and the vertical structure equation takes the Sturm-Liouville form:

$$
\frac{\partial}{\partial z}\left(\frac{1}{S} \frac{\partial}{j z} F_{1}\right)+\Gamma_{1}^{2} F_{1}=0 \quad \text { Eq. II.5 }
$$

with:

$$
\left(F_{1}\right)_{z}=0 \text { at } z=0,-1
$$

where the separation constant, $\Gamma_{i}^{-1}$, is the non-dimensional Rossby Deformation Radius corresponding to the ith mode. We normalize the Fi according to:

$$
\int_{-1}^{c} F_{1 F j d z}=\delta_{i j}
$$

From Sturm-Liouville theory, we know the set of functions [Fi] is complete, and therefore, we can write the pressure $P$ as:

$$
P(x, y, z, t)=\sum \alpha_{1}(x, y, t) F_{1}(z) \quad \text { Eq. II. } 6
$$

where

$$
\alpha_{i}=\int_{-i}^{0} \text { PFidz. }
$$

In general we cannot differentiate with respect to $z$ under the summation sign in Eq. II.6, for in the case of non-homogeneous top and bottom boundary conditions, the series will be non-uniformly convergent over the interval $(-1,0)$. To obtain equations for the horizontal structure functions $\mathcal{\alpha}_{i}$, we employ a Galerkin approach (Finlayson, 1972), 1.e. we operate on Eq. II.3 with:

$$
\left.\int_{-i}^{0} F_{i(E q} \cdot \operatorname{II} \cdot 3\right) d z
$$


The resulting equation for the ith modal amplitude is:

$$
\begin{array}{rlrl}
\left(\nabla^{2}-j^{2}\right)\left(\alpha_{i}\right)_{t} & +\sum_{i=\sum_{i j} Q J\left(\alpha_{j},\left(\sigma^{2}-i_{k} 2 \alpha_{k}\right)+\left(\alpha_{i}\right)_{x}=\right.} \\
& =F_{i}(0) Q w_{e} & \text { Eq. II.7 }
\end{array}
$$

where:

$$
\xi_{i j k}=\sum_{-}^{0} F_{i} F_{j} F k d z
$$

is a coefficient representing the non-linear production of mode $i$ from interactions of modes $j$ and $k$. In later discussions, the evolution equations of Eqs. II.7 will be referred to as the continuous equations.

It is useful to examine the results of a similar procedure on the quasi-geostrophic equations appropriate to a layered model. The nondimensional equation for the pressure in the ith layer may be written as:

$$
\begin{gathered}
{\left[\frac{\partial}{\partial t}+Q J\left(P_{i}, .\right)\right]\left[\nabla^{2} P_{i}+\frac{f_{0}^{2} L^{2}}{g_{i-1}^{\prime} H_{i}}\left(P_{i-1}-P_{i}\right)+\right.} \\
\left.\left.-\frac{f_{0}^{2} L^{2}}{g_{i}^{2} H_{1}}\left(P_{i}-P_{i+1}\right)\right]+\frac{\partial}{d x} P_{i}=\text { (forcing }\right)_{i}
\end{gathered}
$$

where $Q=U /(\beta L 2)$ as before, $\mathrm{H}_{1}$ is the average thickness of layer $i$, and $g^{\prime}=\left(P_{i}-P_{i-1}\right) / !_{0}^{0}$ (see Fig. II.1). As in the continuous equations, we attempt a separable solution to the linearized form of Eq. II.8:

$$
P_{i}=\sum_{j} \hat{\alpha}_{j}(x, y, t) \hat{F}_{j}(i) \text {, }
$$




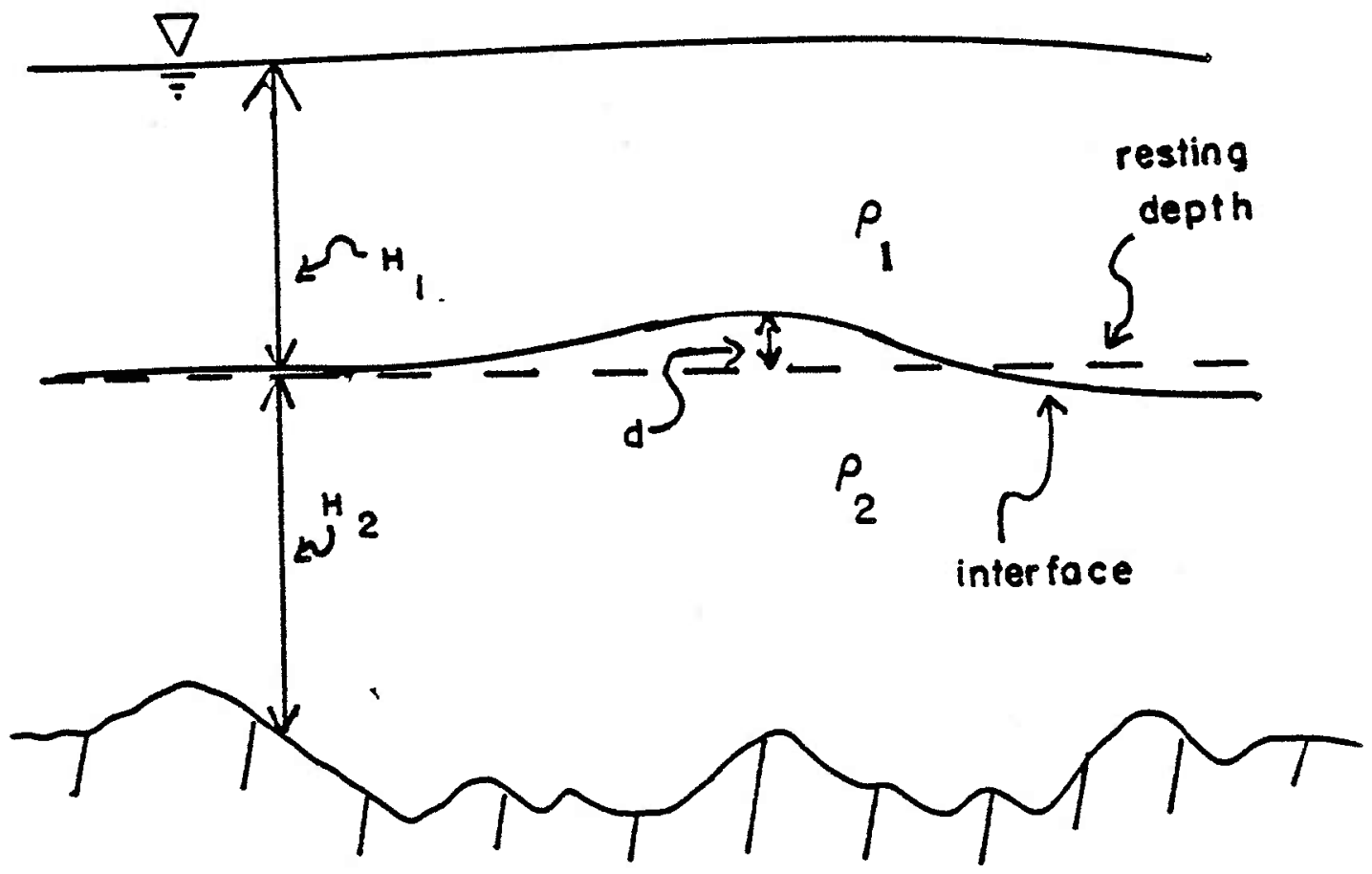

Figure II.1. Schematic Diagram of a Two Layer Ocean

The dashed line represents the configuration of the interface for the resting state and ' $d(x, y, t)$ ' describes displacements of the interface related to geostrophic motion. Also shown are the average layer thicknesses, $\mathrm{H}_{1}$ and $\mathrm{H}_{2}$, and the layer densities $\rho_{1}$ and $\rho_{2} \cdot$ For a continuously stratified ocean, the density is described by the buoyancy frequency $N^{2}(z)$, and ' $d$ ' designates the fluctuations of 1 sopycnals away from the mean state. 
where $\hat{\alpha}_{i}$ represents a horizontal structure function, and $\hat{F}_{j(i)}$ the jth eigenmode in the ith layer, which returns a separability condition in the form of a homogeneous tridiagonal matrix equation for the vector ^ $F_{j}(i):$

$$
\begin{array}{ccc}
\frac{f_{0}^{2} L^{2}}{g_{i}^{1} H_{i}} \hat{F}_{j}(i+1) & -\left[\frac{f_{0}^{2}}{g_{i}^{T} H_{i}}+\frac{f_{0}^{2}}{g_{i-1}^{H_{i}}}\right] \hat{F}_{j}(i)+\frac{f_{0}^{2}}{g_{i-1}^{1} H_{i}} \hat{F}_{j}(i-1)+ \\
& +\hat{\Gamma}_{j}^{2} \hat{F}_{j}(i)=0 . & \text { Eq. II.9 }
\end{array}
$$

We normalize the $\overline{[F} j(i)]$ by:

$$
\sum_{i} \hat{F}_{j(i) \hat{F k}(i) H i}=\delta_{j k} \text { Eq. II. } 10
$$

The horizontal structure equations, governing the $\alpha_{i}$, are:

$$
\begin{aligned}
& \left.\left.\hat{\sigma}^{2}-\hat{r}_{k} 2 \hat{\alpha}_{k}+\sum_{i_{i}} \hat{\xi}_{i j k J} \hat{\alpha}_{i,(\nabla 2}-\hat{r}_{j} 2\right) \hat{\alpha}_{j}\right)+\left(\hat{\alpha}_{i}\right) x= \\
& =\hat{F k}(1) Q w e ;
\end{aligned}
$$

we shall refer to Eqs. II. 11 as the layered equations. In form, Eqs. II. 1 are identical to Eqs. II.7, however there are important, subtle differences between the two involving the modal parameters, $\hat{\xi}_{i j k}, \hat{\Gamma}_{k}$, and $\hat{F}_{k}(1)$ for the layered case, and $\xi_{i j k}, \Gamma_{k}$, and $F_{k}(0)$ for the continuous equations (see Table II.2). Consider the baroclinic mode of a two layer model; all the baroclinic parameters, $\bar{\xi}_{111}, \Gamma_{1}$, and $F_{1}(1)$, 
Table II.3 Layer and Continuous Modal Parameters

After Flierl (1978)

Continuous Two Layer

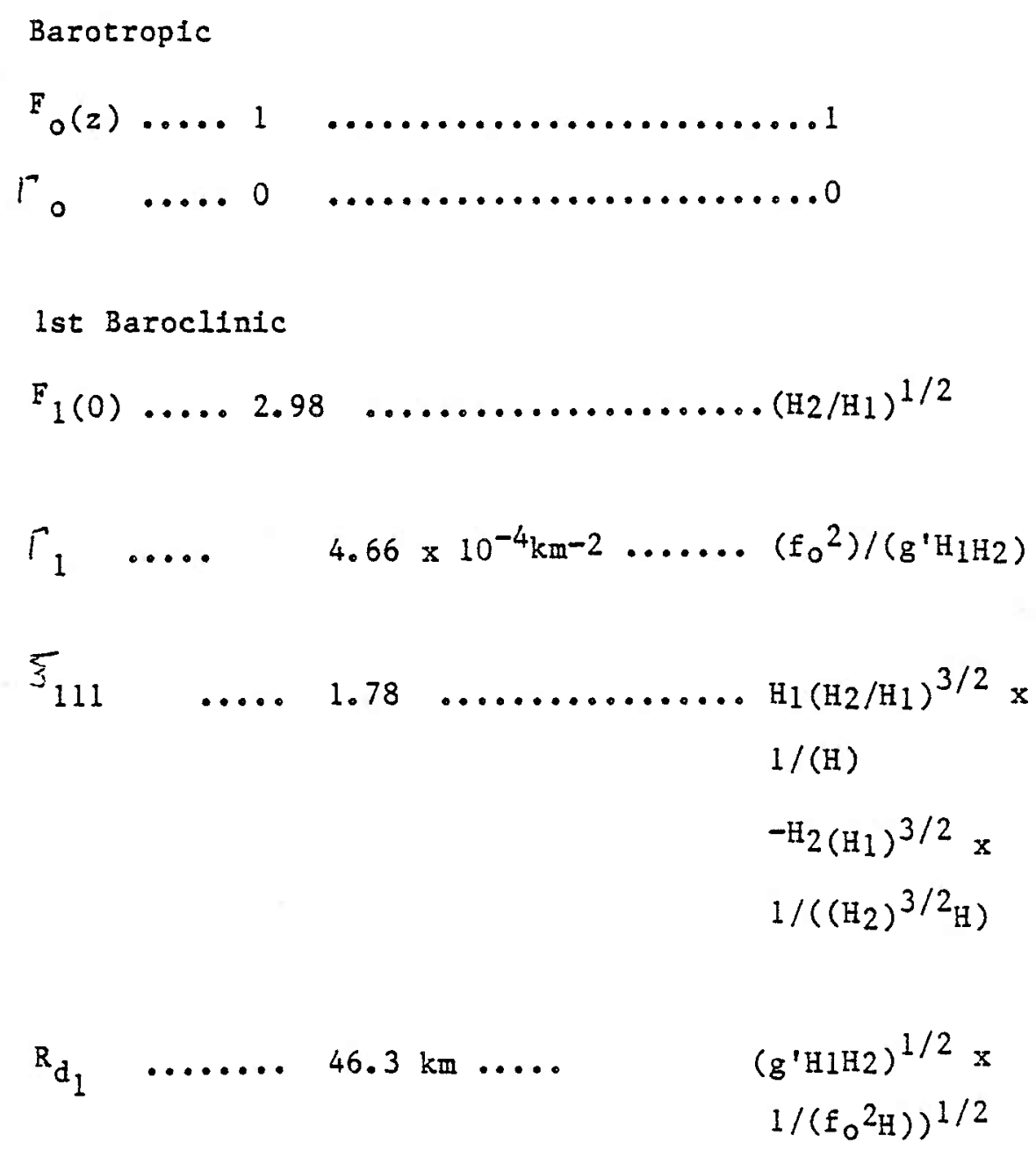


are specified by a choice of one density step, $g^{\prime} l$, and one layer depth, Hl, assuming a value for the total depth $H$. That is, only two of the three parameters are independent. In the analogous two modal case, $\bar{\xi}_{111}, \Gamma_{1}$, and $F_{1}(0)$ are independent, reflecting a greater information content in the continuous equations. Flierl (1978) has shown that the continuous equations are automatically 'calibrated' because all of the information about the mean stratification (in N2) is used to compute the modal structures, and hence the system parameters. In the presence of surface forcing, Eqs. II.7 are more accurate than the layered equations, and will be used in Chapters $V$ and VI which are concerned with forced motion.

Note that if $w_{e}=0$, the number of continous modal parameters in Eq. II. 7 is reduced to two $\left(\xi_{i j k}\right.$ and $\left.\Gamma_{k}\right)$, the same number as in the unforced layered equations. In this case, the layered system is isomorphic to the continuous system and we are free to interpret the calculations in either frame. Generally, the two layered system is more intuitive, so 1 t is this system in which we will interpret the advection diffusion calculations of Chapter III. The conversions between modal amplitudes and layer pressures for the two layer unforced case are given by:

$$
\hat{\alpha}_{0}+\delta^{1 / 2} \hat{\alpha}_{1}=p_{1}
$$

and:

$$
\hat{\alpha}_{0}-j 1 / 2 \dot{\alpha}_{1}=P_{2},
$$

where $\delta=\mathrm{H}_{1 / \mathrm{H}}$. 
In Chapter V, we will need to calculate $d$, the deviation of an isopycnal from its resting depth (see Fig. II.1). The formula we will use is:

$$
d=-P_{z} / N 2=-\left(\sum \alpha_{i}\left(F_{i}\right)_{z}\right) / N^{2}, \quad \text { Eq. II. } 13
$$

which may be obtained by operating on the hydrostatic equation with:

$$
\int_{1}^{\infty}\left[\cdot f_{0}^{2}\left(F_{z}\right) / N^{2}\right] d z \text {, }
$$

and using the quasi-geostrophic equation:

$$
-\mathrm{N}^{2} \mathrm{~d}=\mathrm{b} \text {. }
$$

The Equivalent Barotropic Equation-

We obtain a two degree-of-freedom model if we retain only the two lowest modes in Eqs. II.7. Such a model has been used to study a varlety of oceanic problems (F1ierl, 1978). Under certain circumstances, we may simplify the equations further to a single formula known as the equivalent barotropic equation.

$$
\begin{aligned}
& \left(\nabla 2-\nabla^{2}\right) \alpha_{t}+Q \xi 111 J\left(\alpha,\left(\nabla^{2}-p^{2}\right) \alpha\right)+\alpha_{x}=\quad \text { Eq. II. } 14 \\
& \text { = forcing - dissipation. }
\end{aligned}
$$

In this subsection, we will discuss the unforced $\left(w_{e}=0\right)$ layered equations (recall the isomorphism) to 1llustrate the physical system that Eq. II. 14 describes.

From Eq. II.12, we see that the condition for a resting lower layer, $\mathrm{P}_{2}=0$, is:

$$
\alpha_{0}=S^{1 / 2} \alpha_{1}
$$

However, if $P_{2}=0, E q$. II. 8 with $i=2$ becomes:

$$
\left(P_{1}\right) t=0 \text {. }
$$


Obviously, it is essential for the existence of time dependent flow that the lower layer not be strictly at rest. A proper interpretation of Eq. II.15 is that for weak lower layer flows, the ratio $\alpha_{0 / \alpha_{1}}$ is $O\left(\xi^{1 / 2}\right)$, as occurred in the numerical experiments of McWilliams and Flierl (1979), where the lower layer developed as an incoherent $O(\bar{\delta} 1 / 2)$ wave field. If $P_{1}=0(1)$, we see $\alpha_{1}=0(\delta 1 / 2)$, and therefore, $\alpha_{0}=0(\delta)$. Introducing the rescaled modal amplitudes:

$$
\alpha_{0}=8^{2} \text {, }
$$

and

$$
\alpha_{1}=\varepsilon^{1 / 2} \chi
$$

Into the layer equations, the lowest order in $\delta^{1 / 2}(<<1)$ is:

$$
\begin{array}{ll}
\left(\nabla^{2}-r^{2}\right) \chi_{t}+Q J\left(\chi,\left(\nabla^{2}-r^{2}\right) \chi\right)+\chi_{\mathbf{x}}=0, \text { and } & \text { a. Eq. II. } 16 \\
\left(\nabla^{2} \psi\right) t+Q J\left(X,\left(\nabla^{2}-r^{2}\right) \chi\right)+\psi_{x}=0 . & \text { b. }
\end{array}
$$

We shall be primarily concerned with Eq. II.16.a, the equivalent barotropic equation, which we see, if $w_{e}=0$, is the governing equation for the first baroclinic mode of a two layer fluid with a thin upper layer. Extensions of the present scaling arguments to the case we $\neq 0$ will be made later.

A Discussion of Baroclinic Instability-

The lack of mode-mode transfers excludes baroclinic instability from Eq. II.14. This may be seen by multiplying Eq. II.7, with $i=1$, by $\alpha_{1}$ and area averaging, assuming either no flow at $\infty$ or periodic boundary conditions. We obtain:

$$
\left[\iint_{j}\left(\left(\left(\nabla \alpha_{1}\right)^{2}+\Gamma_{1} 2 \alpha_{1} 2\right) / 2\right) \mathrm{dA}\right]_{t}=\iint \alpha_{1} J\left(\alpha_{0},\left(\nabla 2-\vec{r}_{1}\right)^{\alpha_{1}}\right) \mathrm{dA}, \quad \text { Eq. II. } 17
$$
the baroclinic energy equation, the right hand side of which represents 


\section{Page -27-}

energy conversion between the barotropic and baroclinic modes, or baroclinic instability processes. By previous scaling, the right hand side is $O(F)$ compared to the left hand side, and therefore negligible. While for some applications the lack of baroclinic instability might represent a shortcoming, the problems under consideration in the present thesis are not likely to be strongly affected.

II.c Advection-Diffusion of a Passive Scalar-

If a fluid parcel is convecting a passive tracer, $A$, the evolution of $A$ is governed by:

$$
\mathrm{At}+\underline{\underline{u}} \cdot \nabla \mathrm{A}=\mathrm{D}+\mathrm{K}_{\mathrm{m}} \nabla^{2 \mathrm{~A}} \quad \mathrm{Eq} \cdot \mathrm{II} \cdot 18
$$

where $D$ symbolizes decay and $K_{m}$ molecular diffusivity. If we average $(<\rangle)$ Eq. II.18 in some suitable way, we obtain:

$$
\langle A\rangle_{t}+\langle\underline{u}\rangle \cdot \nabla\langle A\rangle=-\nabla \cdot\left\langle u^{\prime} A^{\prime}\right\rangle+\langle D\rangle+R 2\langle A\rangle,
$$

with coherent small scale transport providing a source for the mean fields. We will employ Fickian diffusion as a turbulent closure:

$$
F_{1}=\left\langle u^{\prime} i A^{\prime}\right\rangle=-K_{i j A x_{j}} ; \quad \text { Eq. II. } 19
$$

therefore, the equation for $A$ becomes:

$$
\langle A\rangle_{t}+\left\langle u_{j}\right\rangle\langle A\rangle_{x_{j}}=\langle D\rangle+\left(K_{i j}\langle A\rangle_{x_{j}}\right)_{x_{i}}, \quad \text { Eq. } \cdot \text { II. } 20
$$

where we have neglected molecular processes.

From field measurements and laboratory work, it is known that turbulent mixing in the ocean is highly anisotropic, due to stratification, and that tracer transport occurs principally along density surfaces. The diffusivity tensor we will use, the only non-zero elements of which are on the main diagonal, models this anisotropy by 
assigning a value to the vertical mixing coefficient which is orders of magnitude smaller than those of the horizontal coefficlents. Hence, on the mesoscale, A is governed by (dropping brackets):

$$
A t+\underline{u_{0}} \nabla A=K(A x x+A y y)+D, \quad E q \cdot I I .21
$$

where we have ignored vertical (across isopycnal) mixing relative to horizontal (along isopycnal) and assumed $\mathrm{Kij}$ to be horizontally isotropic. We shall use only stable tracers, so D will be set to zero.

II. d The Mixed Layer-

The atmosphere forces the ocean via a layer in which small scale turbulent processes are important, and computation of their effect has become an area of much effort. One method consists of explicit computation of the turbulent fluctuations. These so-called deterministic models have proved to be very enlightening, although the required computational effort is large. A second approach is based on the observation that the upper layer is 'well mixed', which allows vertical derivatives to be neglected. Bulk models, as the latter are called, have proved to be reasonably accurate in their prediction of sea surface temperature, and appear to be simple enough to be included in large scale ocean models (Adamec, Elsberry, Garwood, and Haney, 1981).

In this thesis, we shall use a bulk mixed layer model and so will briefly outline the derivation of the bulk equations. Other discussions of the mixed layer can be found in Stevenson (1980) and Muller (1981). 
Conservation of Mass and Thermodynamic Energy-

We model the ocean as a Boussinesq fluid:

$$
\begin{array}{cr}
\nabla \cdot \underline{u}=0, & \text { Eq. II.22 } \\
T_{t}+u T_{x}+v T_{y}+w T_{z}=K_{T} v^{2} T+Q_{z} & E q \cdot I I .23
\end{array}
$$

where $\underline{u}$ represents velocity, $T$ temperature, $K_{T}$ the coefficient of thermal diffusivity, and $Q_{z}$ internal heat sources. Averaging Eq. II.23 and using Eq. II.22 returns:

$$
\left.\overline{\mathrm{T}}_{t}+\overline{\mathrm{u}} \overline{\mathrm{T}}_{\mathrm{x}}+\overline{\mathrm{v}} \overline{\mathrm{T}}_{\mathrm{y}}+\overline{\mathrm{w}}_{\mathrm{T}}=\mathrm{K}_{\mathrm{T}} \overline{\mathrm{v}}^{2 \bar{T}}+\overline{\mathrm{Q}}_{z}-\overline{\left(\mathrm{w}^{\prime} \mathrm{T}^{\prime}\right)}\right)_{z} \cdot \quad \text { Eq. II. } 24
$$

Here we have made the standard assumption that turbulent transfers are greater vertically than horizontally, or that $\left(\overline{\left.u^{\prime} q^{\prime}\right) x}, \overline{\left(v^{\prime} q^{\prime}\right) y}<<\right.$ $\left(w^{\prime} q^{\prime}\right)_{z}$ where $q$ is an arbitrary variable: A similar scaling will occur in all mixed layer equations.

We take the equation of state for seawater to be:

$$
\rho=\Gamma_{0}\left(1-\hat{\gamma}\left(T-T_{0}\right)\right), \quad \text { Eq. II. } 25
$$

where $P_{0}$ is a reference density, To a reference temperature, if the coefficient of thermal expansion for seawater, and we have ignored salinity. Eq. II.25 allows us to convert Eq. II.24 to an equation governing buoyancy:

$$
\begin{gathered}
b=-g\left(v-\Gamma_{0}\right) / P_{0} \cdot \\
\bar{b}_{t}+\bar{u} \bar{b}_{x}+\bar{v} \bar{b}_{y}+\bar{w} \bar{b}_{z}=-\overline{\left(w^{\prime} b^{\prime}\right)_{z}}+k \cdot \overline{2 b}+\overline{B_{O z}} \text {, Eq. II. } 26
\end{gathered}
$$

where $\overline{\left(B_{0}\right) z}$ represents internal buoyancy sources. We suppose that the turbulent fluxes well-mix the upper layer, so that due to the lack of $z$ dependence in the mean state variables, the vertical integration of Eq. II. 26 over the mixed layer depth, h, is trivial:

$$
\left.h(b t+u b x+v b y)=\overline{-w^{\prime} b^{\prime}}\right]_{0}+\overline{\left.w^{\prime} b^{\prime}\right]}-h+B(0)-B(-h), \quad \text { Eq. II. } 27
$$


where we have neglected free surface variations and diffusion, and dropped the overbars on $u, v$, and $b$.

To close this equation in terms of mean variables, boundary conditions on the turbulent buoyancy flux need to be specified. The mechanisms of heat removal from the ocean surface include latent heat loss, sensible heat loss, and black body radiation, all of which may be evaluated using bulk empirical formulae and encapsulated in the form:

$$
\left.\overline{w^{\prime} b^{\prime}}\right]_{0}=r g \tilde{B}(T-T a)+c \quad \text { Eq. II. } 28
$$

where $\widetilde{\beta}$ is an empirical coefficient, Ta the atmospheric temperature, and $c$ a bias of the heat flux deriving from the fact that evaporation can only cool the sea surface. From a least squares regression of air-sea temperature difference and measured surface heat flux, Frankignoul found a value of $\bar{\beta}=10-3 \mathrm{~cm} / \mathrm{sec}$ (personal communication). Also, from analysis of bulk meteorological formulae for surface heat fluxes (such as in Thompson, 1974), $\widetilde{\beta}$ is found to be $1.5 \times 10^{-3} \mathrm{~cm} / \mathrm{sec}$. The calculations performed in this thesis all used $\widetilde{\beta}=10-3 \mathrm{~cm} / \mathrm{sec}$. At $z=-h$, the mixed layer, if $i t$ is deepening, entrains cold water:

$$
\left.\overline{w^{\prime} b^{\prime}}\right]_{-h}=\left(b_{i}-b\right) e, \quad \text { Eq. II. } 29
$$

where $b_{i}$ is the buoyancy beneath the mixed layer and:

$$
e=h_{t}+u h_{x}+v h_{y}+w l_{-h} \text {. }
$$

If the mixed layer is not deepening, there is no heat flux at the interface, so:

$$
\overline{\left.w^{\prime} b^{\prime}\right]}-h=0
$$

Eq. II. 30 .

Using Eqs. II. 28 and 29 in 27 returns:

$$
h\left(b_{t}+u b_{x}+v b_{y}\right)=-\tilde{\beta}\left(b-b_{a}\right)+B_{0}(0)-B_{0}(-h)+\left(b_{i}-b\right) e, E q \text {. II. } 31
$$


where $b_{a}$ is the buoyancy appropriate to the temperature of the air:

$$
b_{a}=+g r\left(T_{a}-T_{0}\right)
$$

Finally, an accurate mixed layer model requires the computation of the density field in the so-called intermediate layer, i.e. the layer extending to a depth of deepest wintertime mixed layer penetration, but which feels direct atmospheric contact for only a fraction of the year. The intermediate layer buoyancy is governed by:

$$
\begin{gathered}
\frac{\partial}{\partial t} b_{i}+u \frac{\partial}{\partial x} b_{i}+v \frac{\partial}{\partial y} b_{i}+w \frac{\partial}{\partial z} b_{i}=-\frac{\partial}{\partial z} \overline{\left(w^{\prime} b^{\prime}\right)}+\frac{\partial}{\partial z} B_{0} \\
\text { Eq. II. } 32
\end{gathered}
$$

Turbulent transport in the intermediate layer is generally weak compared to those in the mixed layer and to other heat transport processes in the intermediate layer (Stevenson, 1980); we shall neglect them. A more interesting comparison is to be made between the strength of the vertical convection of heat, $w\left(b_{i}\right)_{z}$, and radiative heating, $\left(B_{0}\right)$. Evaluating a typical formula for penetrative radiation (Thompson, 1974) at a depth of $50 \mathrm{~m}$, estimating bz by the $\mathrm{N} 2$ value $10^{-6}$ $\mathrm{sec}-2$, and $w$ by $10^{-4} \mathrm{~cm} / \mathrm{sec}$ (see Chapter $\mathrm{V}$ ), we see:

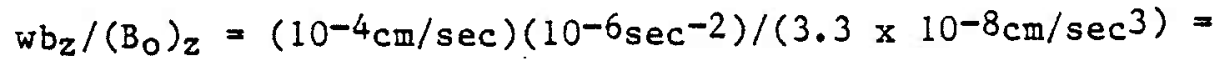

$$
\begin{aligned}
& =3 \times 10^{-3}<1 \text {, }
\end{aligned}
$$
in the intermediate layer.

Momentum Equations-

The averaged momentum equations are (dropping overbars where convenient and neglecting viscosity):

$$
\underline{u}_{t}+\underline{u} \cdot \nabla \underline{u}+\underline{f x u}=-\nabla p-\left(\overline{w^{\prime} \underline{u}}\right) z+b k \cdot \quad \text { Eq. II. } 33
$$


The upper boundary condition on the vertical momentum flux is given by the wind stress:

$$
\left.\overline{-w^{\prime} \underline{u}}\right]_{0}=\left[1 S_{0}^{\prime}\right.
$$

Eq. II. 34

while the stress at the base of the mixed layer consists of both the entrainment of intermediate layer momentum, and the radiation of internal waves:

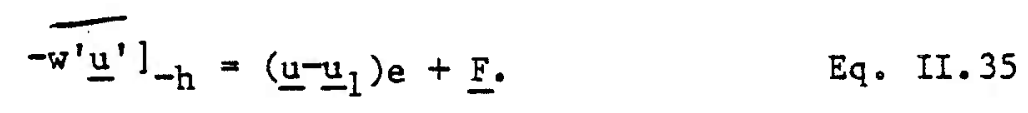

In most mixed layer models, the momentum flux by internal wave radiation is neglected (Nifler and Kraus, 1975) although it is potentially important in determining the amount of energy available for mixing (Kantha, 1975). Bell (1979) estimates that, because of $E$, inertial oscillations are damped out in roughly a week; however, it appears that for low frequencies, $\omega \ll$ fo, momentum loss to $E$ is unimportant (Pollard, 1970). Therefore, we take $E=0$. The vertically integrated horizontal momentum equations are:

$$
h\left(u t+u u_{x}+v u y+w u z-f v\right)=-h P x+\tau_{(x)}+(u 1-u) e,
$$

and Eq. II. 36

$$
h\left(v_{t}+u v x+v v y+w v_{z}+f u\right)=-h P y+\tau_{j}+(v 1-v) e,
$$

and the vertical momentum equation is the hydrostatic balance:

$$
P_{z}=b_{0}
$$

Quasi-Geostrophic Scaling-

For large-scale, low-frequency flows, the inertial momentum terms in Eq. II.33 are O(Rossby number, hereafter Ro) with respect to the Coriolis acceleration, and can therefore at lowest order be neglected. Similarly, a scale estimate of the turbulent momentum transport based on 
the wind stress, when compared to the Coriolis acceleration, is small, leaving a. lowest order geostrophic balance in the upper layer:

$$
\begin{aligned}
& f_{\text {ov }}=P_{X}, \\
& f_{\text {ou }}=-P_{y} \cdot
\end{aligned}
$$

A vertical integration of the hydrostatic balance relates the pressures at any two depths.

$$
\mathrm{P}(z)=\mathrm{P}\left(\mathrm{z}_{0}\right)+\int_{z_{0}}^{\bar{\epsilon}} \mathrm{bd} z .
$$

Eq. II. 37

We shall choose $z_{0}$ to correspond to a depth just below the deepest mixed layer penetration, and therefore a depth governed by quasi-geostrophic dynamics. Roughly speaking, $z_{0}=0(200 \mathrm{~m})$. ' $z$ ' will correspond to a depth within the mixed layer. Substituting for $P$ in the zonal geostrophic balance returns:

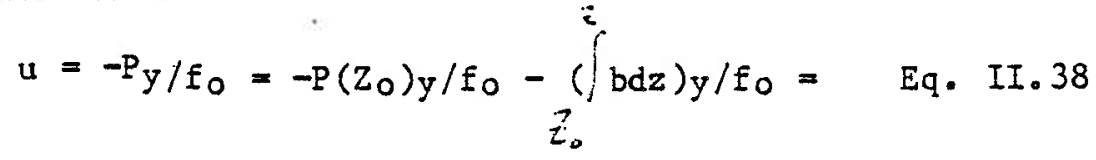

$$
\begin{aligned}
& =u\left(z_{0}\right)-(j \mathrm{bdz}) \mathrm{y} / \mathrm{f}_{0} \text {. }
\end{aligned}
$$

The ratio of the two terms on the right hand side of Eq. II.38 is:

$$
(\Delta b) z_{o} /\left(f_{0} U L\right)=(\Delta b) z_{0} /\left(f_{0} 2 L 2 R_{0}\right), \quad \text { Eq. II. } 39
$$

which will be small if $\Delta b \ll<R_{O} f_{O} 2_{L} 2 / Z_{0}$. A typical Rossby number for $a$ swift, large-scale flow is:

$$
R_{0}=O\left(30 \mathrm{~cm} \mathrm{sec}-1 /\left(10^{-4} \mathrm{sec}^{-1} 6 \times 106 \mathrm{~cm}\right)\right)=.05 ;
$$

therefore, for the ratio in Eq. II. 39 to be small:

$$
\Delta \mathrm{b} \ll((.05) 36 \times 1012) /(10810-4)=1.8 \text {. }
$$

Note that for a Ring, $u=0(100 \mathrm{~cm} / \mathrm{sec})$, and the allowable $\Delta b$ is even larger. In any case, restricting our attention to sea surface buoyancy differences less than $1.8 \mathrm{~cm} / \mathrm{sec}^{2}\left(\delta \mathrm{T}<9{ }^{\circ} \mathrm{C}\right)$, the lowest order, mixed layer, geostrophic balance reduces to:

$$
\underline{u}(z)=\underline{u}\left(Z_{0}\right) . \quad \text { Eq. II. } 40
$$


The surface can support thermal gradients which, because of the thinness of the upper layer, are incapable of seriously perturbing the shallow pressure field.

Ekman Pumping-

The potential vorticity equation obtained from Eq. II.33, which is valid in the mixed layer, is:

$$
\zeta_{t}+u \zeta_{x}+v \zeta_{y}-f_{o w z}+\beta v=\left(\tau_{y}\right) x z-\left(\tau_{x}\right)_{y z} \quad \text { Eq. II.41 }
$$

where $\zeta=v x-u y$, and $\Sigma=\widehat{w^{\prime} \underline{u}^{\prime}}$. From Eq. II.40, we can substitute $\zeta\left(z_{0}\right)$ for the upper layer vorticity and $v\left(z_{0}\right)$ for the upper layer meridional velocity. At $Z_{0}$, the vorticity balance is that of quasigeostrophic dynamics;

$$
\left[\zeta_{t}+u \zeta_{x}+v \zeta_{y}+\beta v\right]_{z_{0}}=f_{0} w_{z}\left(z_{0}\right)
$$

which allows us to rewrite Eq. II. 41 as:

$$
-f_{0 w z}+f_{0 w z}\left(Z_{0}\right)=\operatorname{curl}(\underline{\bar{C}}) z \cdot \quad \text { Eq. II.42 }
$$

Integrating from $Z=0$ to the level surface $z=Z_{0}$ returns:

$$
\begin{aligned}
& -f_{0}\left(w(0)-w\left(Z_{0}\right)\right)+f_{0} w_{z}\left(Z_{0}\right) Z_{0}=\operatorname{curl}(\underline{\tau}(0))-\operatorname{curl}\left(\underline{\tau}\left(Z_{0}\right)\right) \text {. Eq. II. } 43 \\
& \begin{array}{lllll}
1 & 2 & 3 & 4 & 5
\end{array}
\end{aligned}
$$

At depth, turbulent stresses are weak, and we are fgnoring internal wave radiation, hence we can neglect term 5. Applying the boundary condition $w(0)=0$ leaves us with terms 2 and 3 on the left hand side of Eq. II.43. Term 3 represents a correction to the vertical velocity at depth $z_{0}$ due to the quasi-geostrophic divergences in the fluid above it; however, comparing terms 2 and 3 shows:

$$
w_{z}\left(Z_{0}\right) Z_{0} / w\left(Z_{0}\right)=O\left(Z_{0} / H\right)<<1,
$$


and we obtain the classical Ekman pumping upper boundary condition on the interior flow:

$$
w\left(Z_{0}\right)=w(0)+O\left(Z_{0} / H\right)=\underline{k} \cdot \operatorname{curl}(\underline{I}(0)) /\left(f_{0}\right) \cdot \quad \text { Eq. II. } 44
$$

Energy Equation-

As the mixed layer equations now stand, we have 5 equations ( 2 momentum, hydrostatic balance, thermodynamic energy, and mass conservation) in six unknowns $(u, v, p, b, e, h)$. The classical technique for closing this system of equations uses the overall energy budget of the mixed layer, careful derivations of which have been presented in Niller and Krauss (1977) and Stevenson (1980). Here, we shall simply write down the energy equation, and discuss the relative importance of its several components.

Neglecting local storage of turbulent kinetic energy, the bulk energy equation is:

$$
\begin{array}{cccc}
e\left(\left(b-b_{i}\right) h-\left(\underline{u}-\underline{u}_{i}\right)^{2}\right)= & 2 m_{o}|\underline{\tau}|^{3 / 2}+B_{o} h-\int_{-h}^{o} \epsilon d z, & \text { Eq. II. } 45
\end{array}
$$

where $u_{i}$ is the momentum of the intermediate layer, $B_{0}$ the surface heat flux, and $\epsilon$ the dissipation. Term 'a' represents a measure of the energy needed to entrain and mix cold, heavy fluid over the layer's full vertical extent. Term ' $b$ ' is the amount of energy available in the shear at the naviface. Term ' $c$ ' represents direct turbulence generation at the surface by the wind, generally thought of as breaking waves, and 
term ' $d$ ' the flux of potential energy through the surface due to heating and cooling. Finally, the last term represents the dissipation of turbulent energy within the mixed layer, a term whose Importance in turbulent erosion models has been pointed out by Stevenson (1979).

The sum of terms $a$ and $b$ represents the energetic stability of the mixed layer. Consider a simple gravitationally stable two layer system, with the upper layer characterized by velocity $u$ and density $b$, and the lower layer by $u_{i}$ and $b_{i}$. The bulk potential energy of the system, to a depth of $h+\delta h$, is given by:

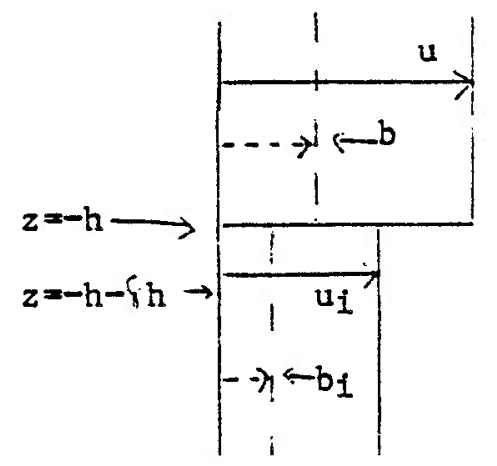

$$
\begin{aligned}
& P E_{i}=-\int_{-(h+5 h)}^{0} z b d z=-\int_{-(h+5 h)}^{-h} b_{1} z d z+ \\
& -\int_{-i n}^{0} b z d z=\left(b-b_{1}\right) h 2 / 2-b i(h+5 h) 2 / 2,
\end{aligned}
$$

and the total kinetic energy by:

$$
\mathrm{KE}_{i}=\mathrm{u}^{2} \mathrm{~h} / 2+\mathrm{u}_{i} 2(\delta \mathrm{h}) / 2 \text {. }
$$

Now suppose that the system mixes itself (?!) to a depth h+ih, and that the new layer is characterized by buoyancy $b^{\prime}$ and velocity $u^{\prime} \cdot b^{\prime}$ and $u^{\prime}$ can be computed from the conservation of heat and momentum:

$$
b^{\prime}=(b h+b i(\delta h)) /\left(h+s^{\prime} h\right) \text {, }
$$

and:

$$
u^{\prime}=(h u+(\delta h) u i) /(h+\delta h) \text {. }
$$

The new bulk potential energy is given by:

$$
\operatorname{PEf}=-\int_{-h \cdot h} b^{\prime} z d z=b^{\prime}(h+\delta h) 2 / 2=
$$

$=(b h+b i(\hat{h} h))(h+\delta h) / 2$, 
and the new kinetic energy by:

$$
K E_{f}=(h+\delta h) u^{\prime} 2 / 2=\left(h u+\delta h u_{1}\right)^{2} /(2(h+\delta h)) .
$$

Note that the change in potential energy:

$$
P E_{f}-P E_{i}=\left(b-b_{i}\right) h \delta h / 2+o\left(\delta_{h}\right)^{2},
$$

is positive; the potential energy has increased because cold fluid has mixed up and warm fluid down. The change in kinetic energy is negative:

$$
\mathrm{KE}_{\mathrm{f}}-\mathrm{KE}_{i}=-\left(\mathrm{u}-\mathrm{u}_{1}\right) \delta_{\mathrm{h}} / 2+O\left(\delta_{\mathrm{h}}\right)^{2},
$$

in agreement with decreasing the shear in the flow. The change in the total energy of the layer is given by:

$$
\delta E_{t}=\left(K E_{f}+P E_{f}\right)-\left(K E_{i}+P E_{i}\right)=\left(\left(b-b_{l}\right) h-\left(u-u_{1}\right) 2\right) \dot{\delta} h / 2 \text {. }
$$

Clearly, if $E_{t}$ is negative, more kinetic energy has been released than potential energy gained. Hence, in a system where:

$$
\left(b-b_{i}\right) h-\left(u-u_{i}\right)^{2}
$$

is negative, a perturbation can draw energy from the shear, grow, and 'mix'. This is the basic dynamic erosion mechanism originally proposed by Pollard, Rhines, and Thompson (1973), in which the shear at the base of the mixed layer is due to the presence of wind driven inertial oscillations.

The time rate of change of total energy is:

$$
\begin{aligned}
& d / d t(E)=\delta E / \delta t=\left(\left(b-b_{i}\right) h-\left(u-u_{i}\right) 2\right) \delta h /(2 \delta t)= \\
& \left(\left(b-b_{i}\right) h-(u-u i)^{2}\right) e / 2, \quad \text { Eq. II. } 46
\end{aligned}
$$

which is a one-dimensional version of the right hand side of Eq. II.45. The only effect on Eq. II.46 of two dimensionality would be the inclusion of a $\left(v-v_{i}\right) 2$ term. Thus, if Eq. II. 46 is negative, we expect mixing to occur, and drive the system back to a state of dynamic stability. If it is positive, 1.e. if there is insufficlent kinetic energy in the shear to generate a mixing event, mixing will occur only 
if energy is transported into the region of the mixed layer base. The terms on the right hand side of Eq. II.45 describe this transport and identify the sources as wind wave breaking and thermal convection, both of which we will neglect. There currently is a difference in opinion amongst mixed layer modelers as to whether it is appropriate to ignore these effects, so we now marshal our relevant arguments.

Wind Wave Breaking and Penetrative Convection-

Recently, direct observations of upper layer turbulent dissipation have been ade and numerical experiments which resolve turbulence have been performed, and some insight into the balance of the dissipation and energy generating mechanisms has been gained. For example, Klein and Coantic (1981) found that the surface wave turbulent field was largely dissipated in the upper few meters, and for mixed layers deeper than about $10 \mathrm{~m}$, inclusion of wave breaking made no noticeable difference in the evolution of the system. Similarly, Gargett, Sanford, and Osborn (1980) noted an increased dissipation in the upper $10 \mathrm{~m}$ of the ocean, which they interpreted as a loss of wave driven energy. Thompson (1981) demonstrated that the energy in the upper layer caused by a random field of whitecaps is strongly surface trapped, and conjectured that the most important property of breaking waves might well lie in their ability to mix wind momentum downwards. Hence, we shall equate term ' $c$ ' of Eq. II.45 to a fraction of the total energy dissipation.

As to penetrative convection, Gargett, Sanford, and Osborn observed that the energy of descending cold water plumes is dissipated prior to 
reaching the mixed layer base, and thus does not assist in deepening. The experiments of Klein and Coantic also exhibit a tendency for buoyant energy production to be balanced by dissipation, although under weak winds and strong cooling, an additional few meter deepening in a thirty meter layer was noted. Similar small increases in numerical mixed layer depths, due to penetrative convection, have been noticed by Mellor and Durbin (1975). Finally, comparisons of model predicted and observed sea surface temperature are generally better when using models without penetrative convection (Gill and Turner, 1976). Therefore, we shall assume that the surface potential energy flux is balanced by dissipation.

The Froude Number Closure and Its Value-

The remaining terms in the energy equation are:

$$
e\left(\left(b-b_{i}\right) h-(\underline{u}-\underline{u} i) 2\right)=\int_{-h}^{\epsilon^{\prime} d z} \quad \text { Eq. II. } 47
$$

where $\epsilon^{\prime}$ is the dissipation left after the above balances have been removed. For $\epsilon^{\prime}=0$, Eq. II. 47 reduces to either the Pollard, Rhines, and Thompson mixing closure:

$$
F=\left(\left(u-u_{i}\right)^{2}+\left(v-v_{i}\right)^{2}\right) /((b-b i) h)=1, \text { a. Eq. II. } 48
$$

or:

$$
\text { e }=0 \text {, b. }
$$

and is the energetic closure used in this thesis. We implement Eq. II. 48 by using ' $b$ ' if $F<1$, and ' $a$ ' otherwise. Note, Price, Mooers, and $\operatorname{Van}$ Leer (1978) suggest that $F=.6$. We have opted to use $F=1$ on the basis of Thompson (1976), who tested a mixed layer model based on 


\section{Page $-40-$}

Eqs. II.48 against various other models and found it returned the highest coherence between predicted and observed SST.

II.e Numerical Techniques-

We have employed double Fourier expansions and spectral methods (Gottlieb and Orszag, 1977) when necessary to perform numerical solutions to the quasi-geostrophic equations. Time stepping was carried out using a leap frog scheme with impliclt formulation of the viscous terms; the computational mode was suppressed by substituting a modified Euler time step at every 50th iteration (Roache, 1977). The remaining numerical calculations are referenced within the text. Finally, a fraction of the numerical calculations reported in this thesis are essentially repeats of some earlier numerical studies conducted by McWilliams and Flierl, the only difference belng that they employed a finite difference technique. In Chapters III, $V$, and VI, we have referred to these calculations as McWilliams and Flierl's calculations, although, technically speaking, they have been performed by the author. 
CHAPTER III. PARTICLE TRAJECTORIES IN

NUMERICAL GULF STREAM RINGS

\section{III.a Introduction-}

There is abundant chemical, blological, and physical evidence that Gulf Stream Rings produce a sizeable net Lagrangian transport (the Ring Group, 1981), in which an individual Ring carries a volume of water. Given the contrast in most oceanographically interesting quantities across the Northern Atlantic Gulf Stream, Ring transport is a potentially important component in the maintenance of North Atlantic tracer distributions.

Outside of the Ring 'trapped' fluid, we have evidence from satellite photographs of the sea surface temperature fields that particles undergo sizeable excursions. Warm core Rings apparently pull filaments of warm and cold water into the slope water during their Interactions with the Gulf Stream or the Shelf-Slope Water front. A fraction of these filaments, or 'streamers', are observed to extend fully across the Slope water, and directly connect the Gulf Stream to the Shelf. The implications with respect to the heat and chemical budgets of the Slope are obvious, although to date no quantitative streamer-flux estimates have appeared in the literature. Cold Rings are observed to interact with the surface temperature expression of the Gulf Stream in a similar manner, pulling filaments of warm water into the Sargasso. 
Table III. 1 Symbols and Definitions

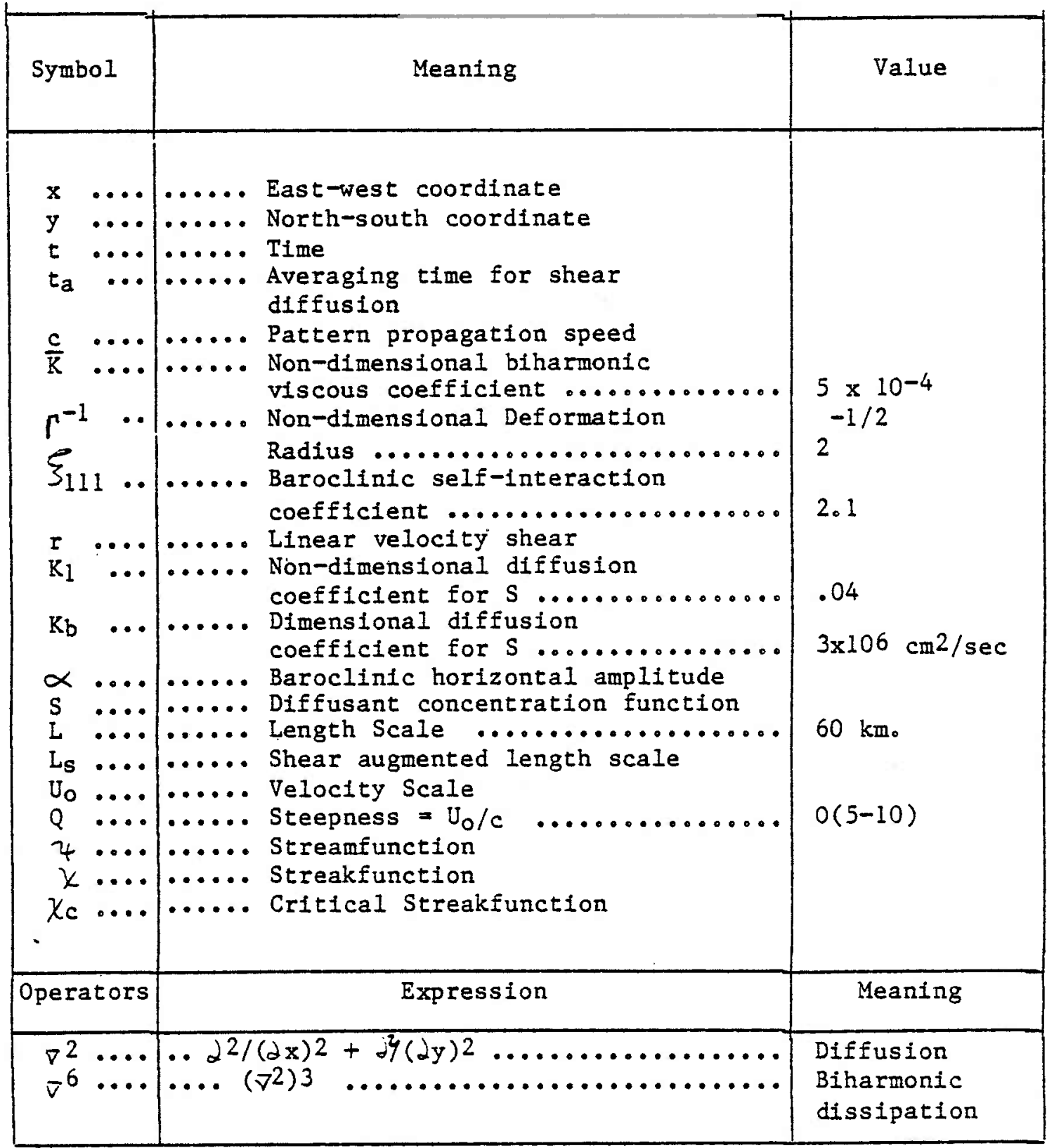


In order to properly account for the effects of Rings on the various budgets, we must first understand their mass transport properties, and in the present chapter, we will compute the particle trajectories associated with a numerical Gulf Stream Ring. In addition, we will discuss a series of advection-diffusion experiments with a view towards understanding how fluid may be exchanged between the trapped zone and the exterior.

The first theoretical pictures of Ring particle trajectories were obtained by Flierl (1981), in which he computed the streaklines associated with a steadily-propagating, axisymmetric pressure pattern. Trapped zones of fluid, propagating with the Ring, arose in this calculation as a consequence of the strong nonlinearity of the flow. For Rings, the 'steepness', $U / C$ where $U$ is a scale for the particle velocities and $c$ the pattern propagation speed, is of order 10 . Outside of the trapped zone, particle trajectories were characterized by meridional excursions on the scale of the Ring (see Fig. III.1). A weakness of Flierl's calculation derives from the fact that his study was purely kinematic. For example, the velocity field he employed, although suggested by data (01son, 1980), is not a solution to the equations of motion; even though it kinematically resembles a Ring, one must question on dynamical grounds the particle trajectories so computed. Also, the shape of those particle trajectories do not agree well with those suggested by satellite surface temperature observations. The numerical Ring model we will employ will evolve subject to the conservation of potential vorticity, and therefore will be dynamically consistent. We shall also see that its particle 


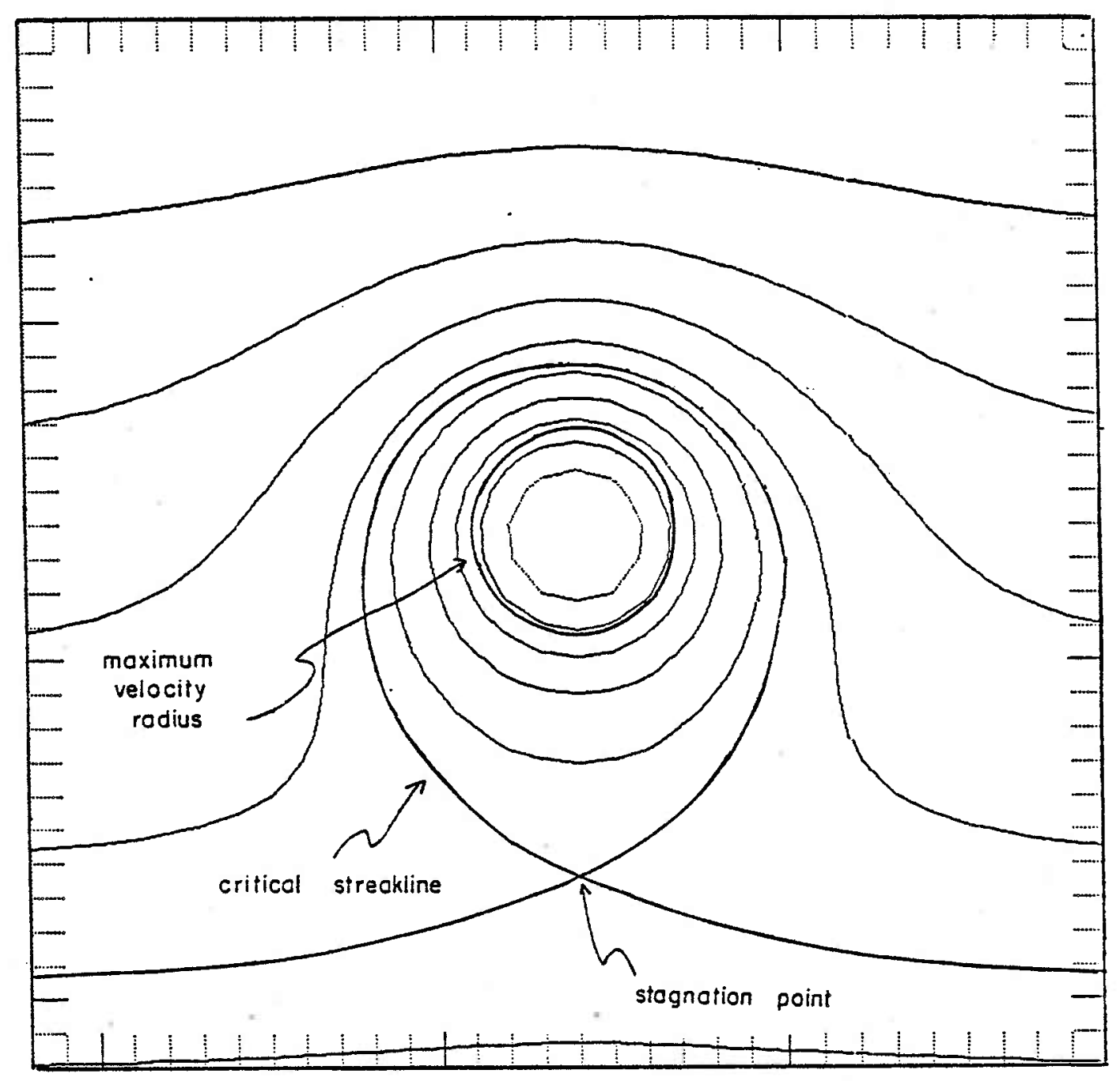

\section{Figure III.1. Streaklines}

Here we have plotted the streakfunction, $x=4+c y$, appropriate to 01son's model streamfunction. Note the critical streakilne, stagnation point, and trapped zone. In this figure, the steepness, $Q$, equals 10; if $Q$ were less than one, all three features would disappear. 
trajectories are in better agreement with observations. Still, most of the interesting features of Flierl's analysis will appear in the dynamical Ring, reflecting that his assumptions of steady-propagation and permanent form are apt.

Ring Mode1-

Many quasi-geostrophic models of Ring structure have been proposed (Flierl, Larichev, McWilliams, and Reznik, 1980) although perhaps the most successful Ring simulations were performed by McWilliams and Flierl (1979). The appealing feature of their model (numerical) is that the pressure field evolves as a 'monopole', which is in agreement with field observations of the baroclinic structure of a Ring (whether the barotropic component of a Ring also has a monopole character is presently unknown). We will employ their equivalent barotropic Ring model, which was governed by Eq. II.14:

$$
\left(\nabla^{2}-\Gamma^{2}\right) \alpha_{t}+Q \xi 111 J\left(\alpha,\left(\nabla^{2}-\Gamma^{2}\right) \alpha\right)+\alpha_{x}=\mathrm{K} \nabla 6 \alpha \text {. Eq. II. } 14
$$

In the next section, after a brief review of Flierl (1981), we will extend his results to include diffusion. In section c, we will discuss a series of numerical experiments involving the advection-diffusion of a passive tracer by McWilliams and Flierl's dynamic Ring and make comparisons with the previous kinematic results. Finally, we will present some simulations of the often observed Ring/Shelf-Slope Water front interactions. 


\section{III.b Kinematic Models-}

Given a steadily propagating streamfunction of the form:

$$
\theta(x, y, t)=\psi(x-c t, y), \quad \text { Eq. III. } 1
$$

Flierl demonstrated that the streaklines, $X$, of particle motion are given by:

$$
x=\psi+c y . \quad \text { Eq. III. } 2
$$

The axisymmetric function:

$$
\begin{array}{lrl}
\Psi=U_{O L}(1-\exp (-3(r-L) / L))+U_{O L} / 2 & r>L \\
\Psi=U_{0}\left(x^{2}+y^{2}\right) /(2 L) & r<L, & \text { Eq. III. } 5
\end{array}
$$

where $L$ denotes the radius of maximum swirl speed, was found by 01son (1980) to accurately describe the streamfunction of Ring Bob, observed during the cyclonic Ring experiment, 1977. Using this function (following Flierl, we will slightly modify Eq. III.5 by ignoring the '3' in the exponential) in the definition of $X$ returns:

$$
\begin{aligned}
& \chi=U_{O L}(1-\exp (-(r-L) / L))+U_{0} L / 2+c b \quad r>L \quad \text { a. } \\
& \text { Eq. III.\$ } \\
& \chi=U_{0}\left(a^{2}+b^{2}\right) /(2 L)+c b=\quad r<L \\
& =U_{0}\left(a^{2}+\left(b+c L / U_{0}\right)^{2}\right) /(2 L)-c^{2 L} /\left(2 U_{0}\right) \quad b .
\end{aligned}
$$

Eq. III.4.b is an equation for a circle centered at position $\left(0,-c L / U_{0}\right)=\left(0, Q^{-1} L\right)$ relative to the center of the Ring. Clearly, if this point lies within the radius of the maximum velocity, $L$ (i.e. $Q-1<1)$, those circles close upon themselves, and regions of trapped fluid will result. The parameter $Q=U_{O} / c$, controlling the existence of closed contours, measures flow steepness and the condition that there be closed contours, $Q>1$, demonstrates that particle trapping is a kinematic consequence of strongly nonlinear, coherent flow. The finite volume of 
trapped fluid is delimited by a critical streakline, $\chi_{c}$, outside of which the streaklines no longer close. At the apex of $\chi_{c}$ is a 'stagnation point' where, in a frame moving with the Ring at speed $c$, $\underline{u}=0$. In a fixed frame, where we perceive the Ring as moving west at speed $c$, the stagnation point occurs where the fluid velocity identically matches the pattern velocity, $\underline{u}_{\text {fixed }}=(c, 0)$.

In Fig. III.1, we plot the streakline contours associated with Eq. III.4 for a steepness value, $Q$, of 10 , corresponding to a warm Ring propagating westward at a speed of $5 \mathrm{~cm} / \mathrm{sec}$ with anti-cyclonic swirl speeds of $50 \mathrm{~cm} / \mathrm{sec}$. The volume of fluid associated with the trapped zone is roughly three times that of the Ring as defined by the radius of maximum velocity, and the trapped zone shape is asymmetric to the north and south. The equivalent picture for westward propagating cold Rings may be abstracted from Fig. III.1 by switching north for south.

From Fig. III.1, we see that particles just north of the critical streakline are displaced strongly to the north as they move around the Ring, while those near to the northern edge of the Ring undergo much less dramatic meridional excursions. Thus, the fluid develops strong shears near the northern edge of the Ring, which acts to distort material lines of fluid. An example of this is shown in Fig. III.2, where we plot a history of several material lines as affected by the streakline field in Fig. III.1. 


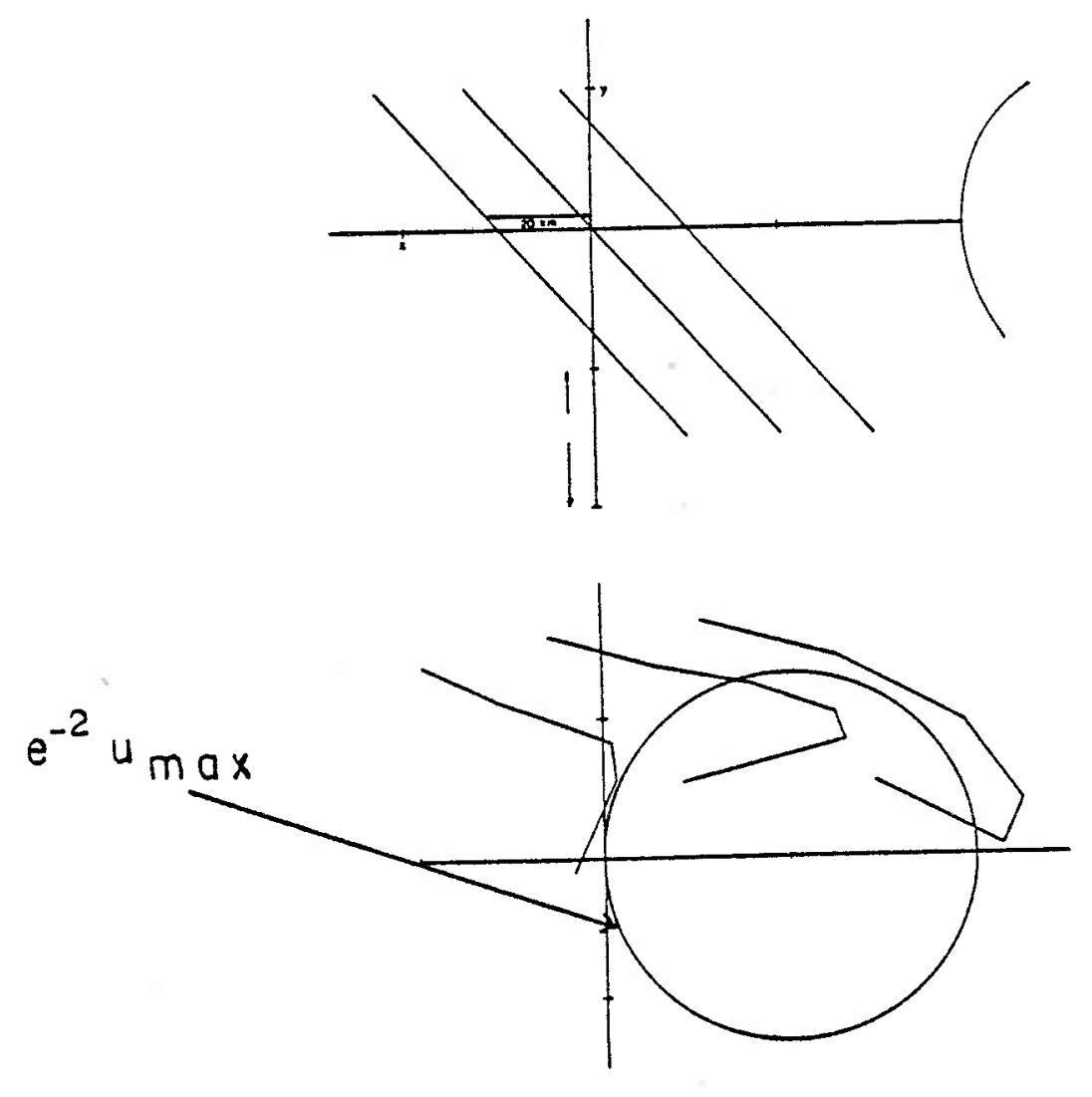

(a)

Ring

Center

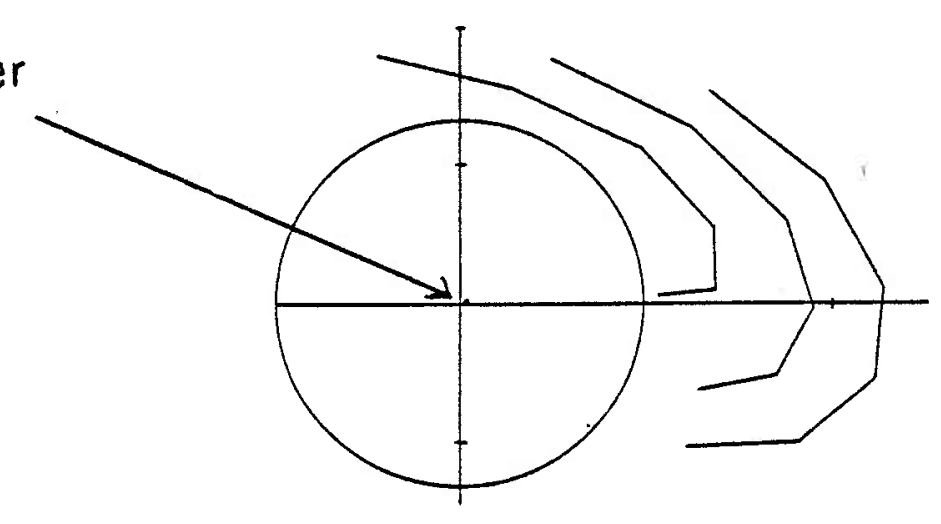

(b)

(c)

Figure III.2. The Effect of a Ring on Material Lines

Here we demonstrate that, in the vicinity of a Ring, the fluid develops strong shears. We have plotted the relative orientation of three lines at various stages of Ring interaction. In (a), the Ring is far away, in (b), the lines are in the midst of the Ring, and in (c), the Ring has passed. Marked are the Ring center, and the radius where the velocities are $e^{-2}$ of their maximum. 
Tracer Diffusion in Kinematic Models-

Consider now the problem of advection-diffusion of a tracer $S$, with the advection provided by the kinematic Ring model of Eq. III.3. We shall model diffusion according to Fick's law:

$$
\mathrm{F} \operatorname{lux}=-\mathrm{K}_{\mathrm{b}} \nabla_{\mathrm{S}}
$$

where $K_{b}$ is the exchange coefficient pertaining to $s$. Under these circumstances, the appropriate equation to solve, in the frame of the Ring, is Eq. II.22, with the advection field given by $\chi_{\text {, }}$

$$
S_{t}+J(X, s)=k_{b} \nabla^{2} s . \quad \text { Eq. II. } 22
$$

Scaling time by $L / C, X$ by $U_{0} L$, and $x$ and $y$ by $L$, the non-dimensional form of Eq. II. 22 becomes:

$$
S_{t}+Q J(X, s)=K_{1} \nabla^{2} s \quad \text { Eq. III.7 }
$$

where the steepness number, $Q=U_{0} / c$, is of order 10 , and $K_{1}^{-1}=$ $\mathrm{CL} / \mathrm{K}_{\mathrm{b}}$ is a Peclet number. For $\mathrm{K}_{\mathrm{b}}=0\left(10^{6} \mathrm{~cm}^{2} / \mathrm{sec}\right.$ ) (Needler and Heath, 1975), $\mathrm{K}_{1}=(.04)$, thus the oceanographically interesting parameter range corresponds to a weak diffusion/strong advection limit.

Tracer Homogenization on Closed Streamlines-

There are some interesting ramifications of Eqs. II.22 and III.7 when applied to regions of closed $X$, with regards to the short time evolution of an arbitrary initial condition. An initial point of dispersant imbedded in a linear shear flow, $u(x)=r x \underline{1}$, will spread horizontally as:

$$
\mathrm{L}_{s} \simeq\left(\mathrm{K}_{b} \mathrm{r}^{2} \mathrm{t}^{3}\right)^{1 / 2}
$$


(Csanady, 1975; Young, 1981). The mechanism involved is shear dispersion, i.e. diffusion spreading the material across the flow, allowing the advection field to enhance the downstream transport. If we suppose that this same shear-augmented diffusion model applies in a local sense, we see that diffusion will tend to force arbitrary initial conditions towards uniformity on closed streaklines. Scaling the shear in the closed $\mathcal{X}$ regions by $U_{0} / L$, the spread, $L_{s}$, of the initial point source, will become of order $L$, and therefore nearly uniform over the closed contour, at a time:

$$
t_{a}=\left(L_{0} / K_{b}\right)^{1 / 3} \mathrm{~L} / U_{0}
$$

For scales appropriate to Rings, this time is:

$$
t_{\mathrm{a}}=[(6 \times 10650) /(3 \times 106)]^{1 / 3}(6 \times 106 / 50)=6.1 \text { days. }
$$

An exception to this rule occurs for solid body rotation, in which case the time scales are controlled by diffusion:

$$
t_{d}=L 2 / K
$$

In Fig. III.3, we present a numerical example where both processes are occurring. In this simulation, velocity shear is concentrated near the edges of the trapped zone, while the center is characterized by a constant rotation rate. Note that by day $14,0\left(2 t_{a}\right)$, the dispersant has homogenized near the edge of the trapped zone, but not in the center. Thus, within the trapped zone, we understand the processes which will act on the dispersant, both of which spread it over the closed contours, and in the presence of shears, force it towards homogeneity along $\mathcal{X}$. Therefore, with little loss of generality, we have chosen inftial conditions like $S(x, y, t=0)=f(X)$ for those advection-diffusion experiments in which the initial blob was located inside the trapped zone. 


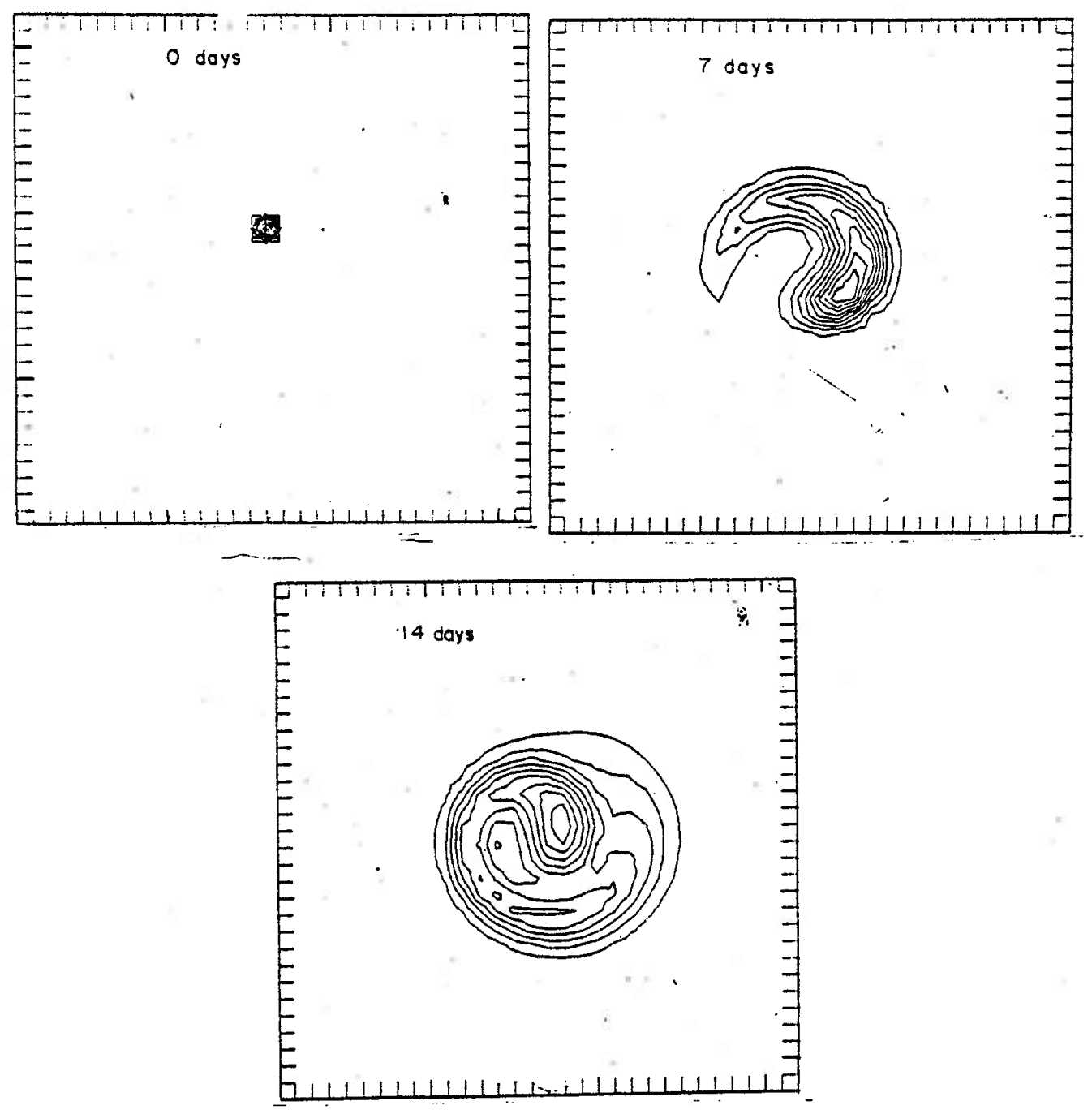

Figure III.3. An Example of Tracer Homogenization

Here are the results of a numerical integration of Eq. III.7, using $X$ as shown in Fig. III.1. Note that this velocity field is composed of solid body rotation out to the maximum velocity, followed by an exponential decrease. All of the shear in the velocity field is located near the critical streakline, and it is there that the tracer has homogenized. In the region of solid body rotation, the time scale for homogenization is the diffusive time scale, which is a much slower process. 
of course, $S$ at the critical contour must be consistent with the external concentration of dispersant, as reference to Figs. III.4 demonstrates. In this experiment, $S$ is assigned the value 1 inside the trapped zone, and zero elsewhere. As time progresses, a strong non $X$-dependent component of $S$ develops on the time scale $t a$, which forces the $s$ on $\chi_{c}$ towards zero in accord with the external conditions. Note as well that the shape of the evolving $S$ contours indicates that the dominant in-flux of $S$-free water occurs near the western edge of the stagnation point. A similar statement applies to the trapped zone-to-exterior fluxes. For example, in Fig. III.5, we exhibit the results of a numerical integration of Eq. III.7 subject to the initial condition:

$$
S(x, y, t=0)=\exp (-(r / 60 \mathrm{~km}) 2)
$$

where the radial distance $r$ is measured relative to the center of the trapped zone. As time progresses, $S$ appears to exit the Ring near the stagnation point, and is left behind in a thin tail. This experiment was repeated with different values for $Q$ and $K$, with the result that the larger (smaller) the $K(Q)$, the broader the tail with respect to the trapped zone. Smaller (larger) K (Q) produced thinner tails. In all cases, exterior $S$ was located dominantly to the south of the trapped zone.

Although both out and in-flux of material becomes apparent near the stagnation point, it is important to note that exchange is occurring all along the extent of the critical contour. We notice the anomalous $S$ values near the stagnation point, because it is there that the streaklines diverge, allowing the exterior dispersant to advect away 

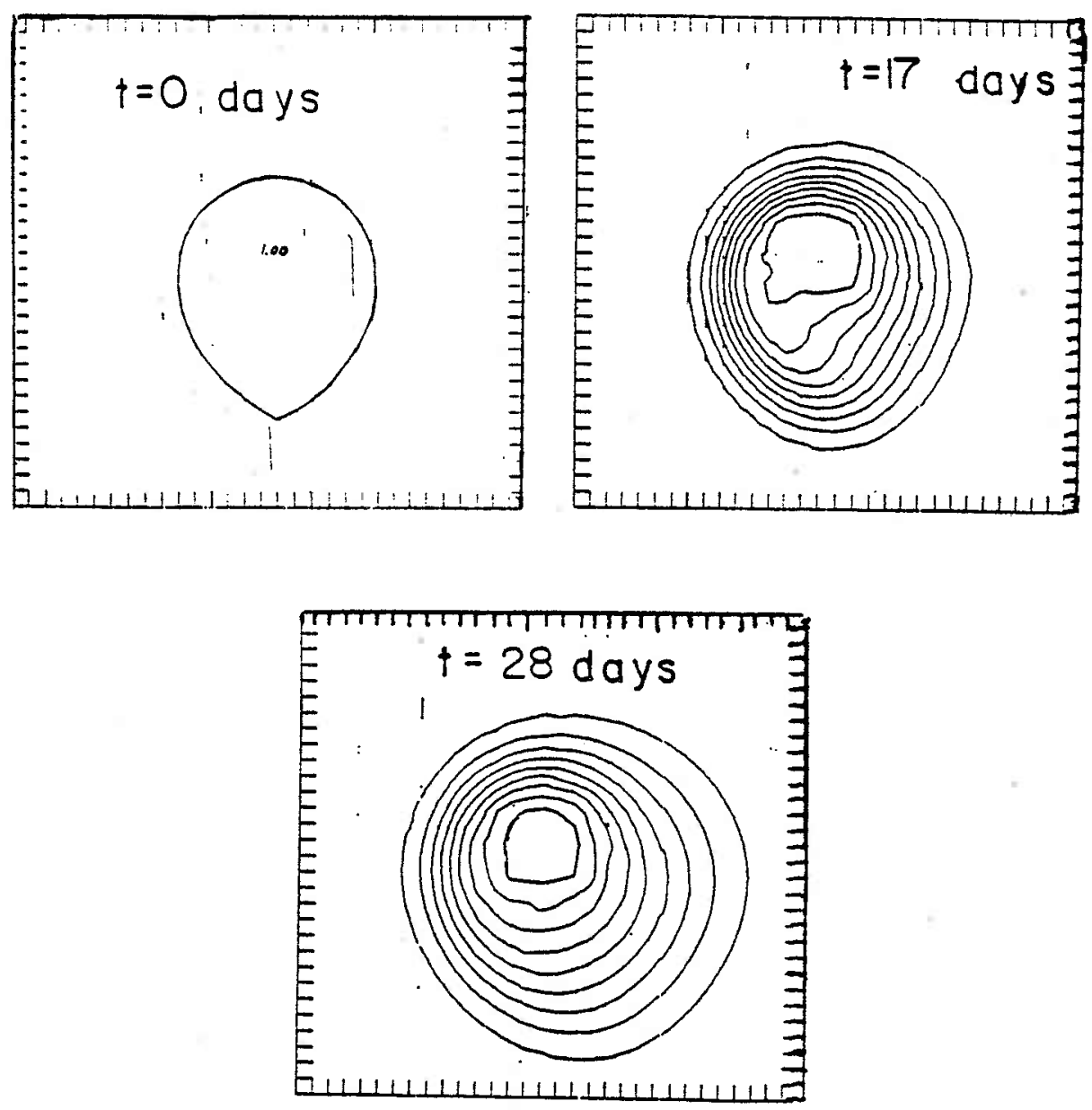

Figure III.4 Boundary Effects on Tracer Homogenization

The contours in these plots are of dispersant concentration, $S$. The initial condition for this series consisted of uniform material $(S=1)$ Inside the trapped zone and $S=0$ outside. As the pattern evolves, note the region of weak $S$ developing just to the north of the stagnation point, an indication of an influx of 's-free' water. Note as well that although the initial condition of the present simulation was a function only of streakline, $S(x, y, 0)=f(X)$, the field develops a strongly non-streakline component on a time scale of ta. The exterior 'S-free' water is forcing the dispersant on the critical streakline to zero, and in turn, interior $S$ is bought by shear dispersion to a functional dependence on more in accord with the exterior. The result about shear dispersion, quoted in the text, and its applications imply that the initial condition must meet the exterior condition at the critical contour. 


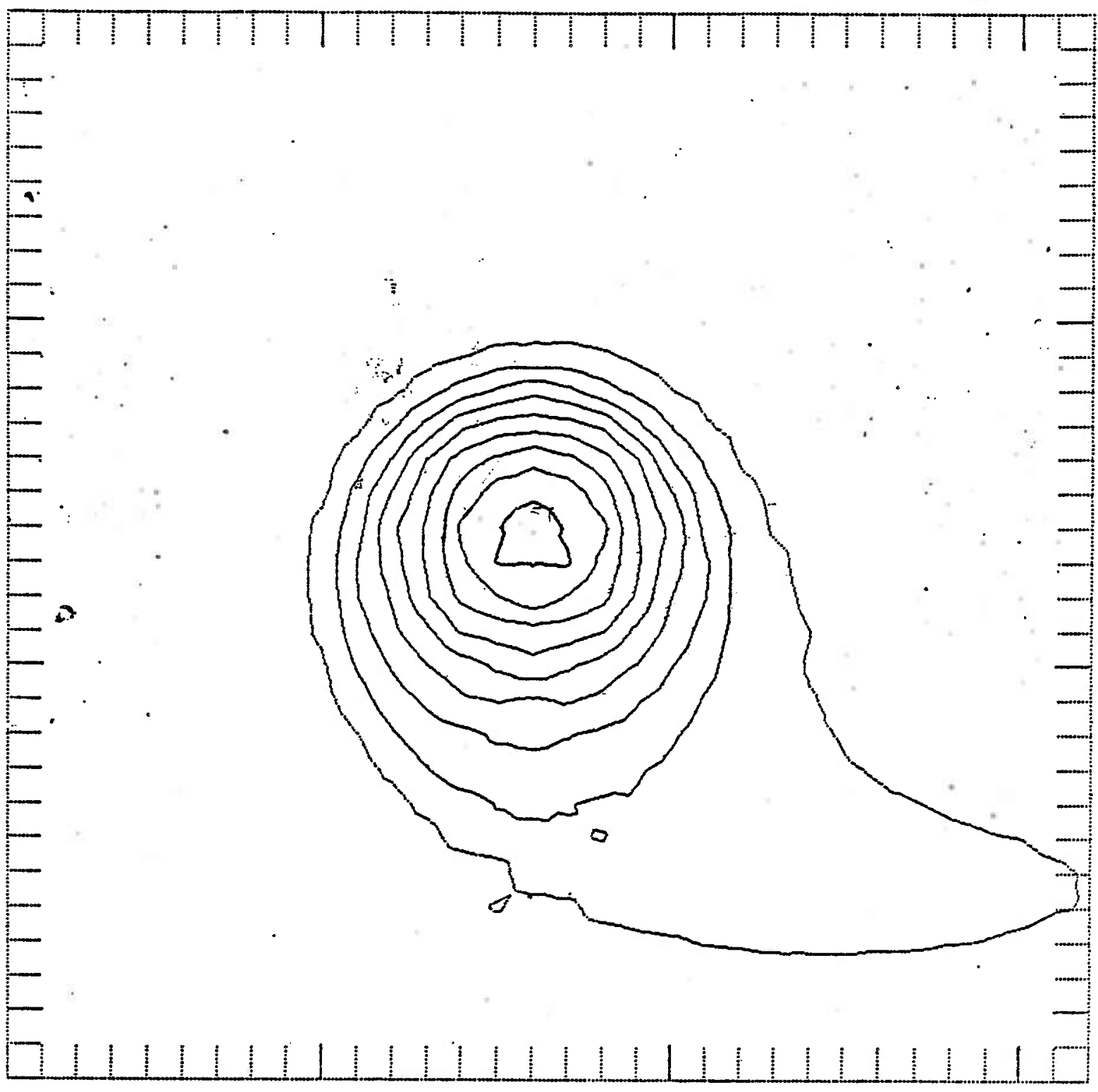

Figure III.5. Advection-Diffusion using the Kinematic Model

Plotted are the results of an advection-diffusion experiment using the streakfunction of Fig. III.1. This experiment began with a Gaussian initial condition of scale $60 \mathrm{~km}$, and shown is $\mathrm{S}$ at day 113 . Here $\mathrm{K}=.04$ and $Q=10$. Note that the dispersant fills out the trapped volume, and exits the trapped zone in a thin tail centered on the critical contour. 
from the Ring, and the S-free waters to invade the trapped zone (see F1g. III.1).

From these simulations, we see that observations of Ring-exterior exchange will largely be confined to the area of the stagnation point, which for a warm (cold) Ring occurs to the south (north) of the radius of maximum velocity. It is interesting that this behavior, particularly tail formation, is often observed in satellite pictures of warm core Rings (see Fig. III.6). Specifically, three of the four satellite warm core images in Fig. III.6 have tail-like features. Also, we see that the cold tongues of water from the Shelf generally end near the southern edges of the Rings.

By this simple model, we have successfully modeled the observed Ring characteristics of a trapped zone and tail formation. A less successful aspect of this model comes from its east-west symmetry. The streaklines suggest that the fluid which ends up at the southeastern side of the stagnation point originates near the critical contour, or to the southwest of the Ring. Satellite photographs of the so-called 'streamers' of cold water, which emanate from the Shelf-slope Water front, suggest that this same fluid originates more to the west of the Ring (see Fig. III.6.d). Finally, note these simulations imply that the fluid which is most likely to exchange with the trapped zone is that to the southwest near the critical contour. The shape and size of the probability distribution for exchange is dependent on $k$, which we do not know, therefore we have not attempted to quantify this statement. However, it will be a useful qualitative remark to compare with results in the coming section. 
Figure III.6. Satellite Observations of Rings

Here we show several satellite photographs of SST in the vicinity of warm core Rings. The Rings in $a, b$, and $c$ have tail-like warm water streams following behind them, as suggested by our model. There is also evidence of asymmetric particle trajectories. Note that the temperature patterns to the left of the Ring are generally broader than those to the right, indicative of a slower northward motion ahead of the Ring. Compare the patterns with the dynamic streaklines of Fig. III.9. 


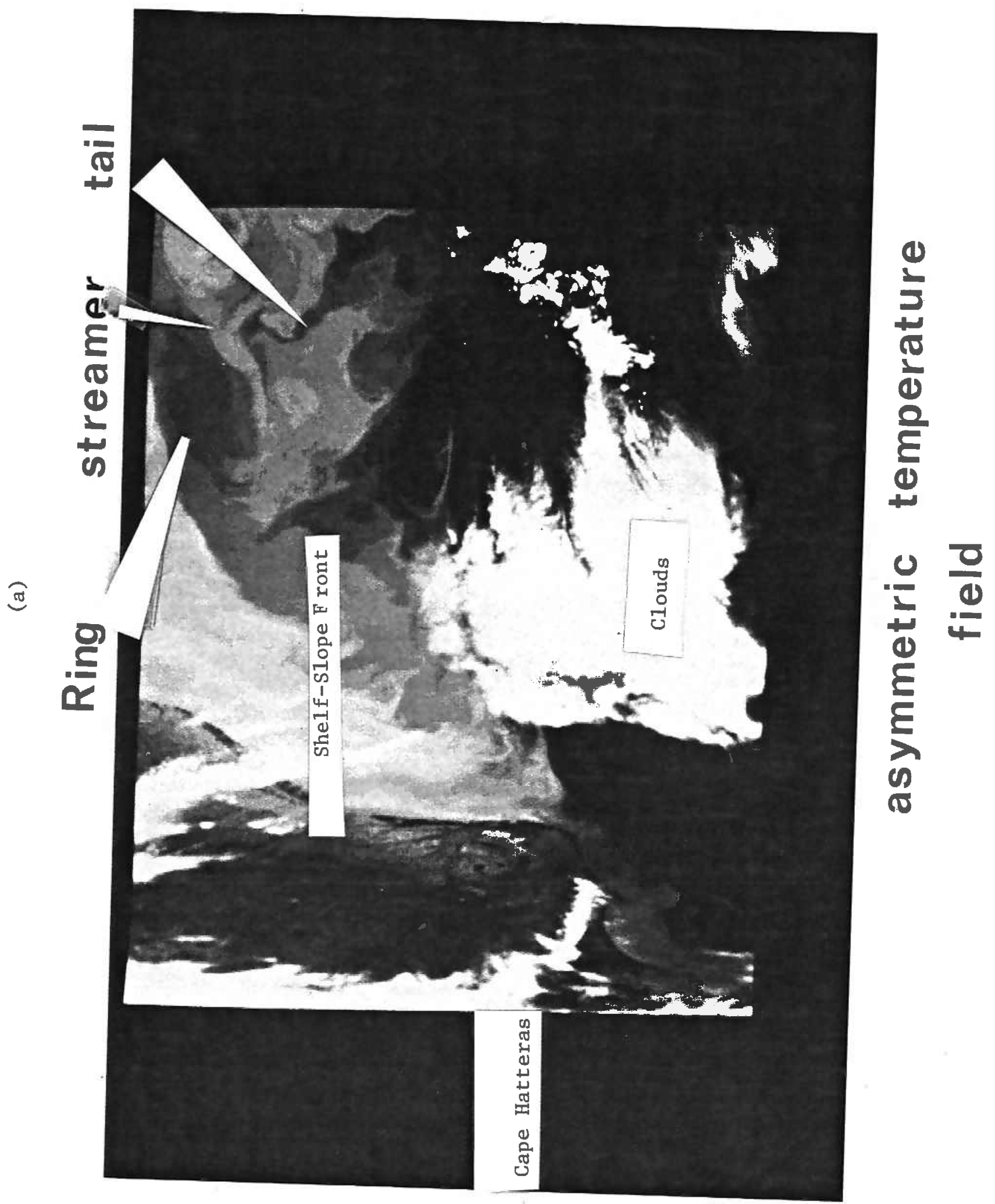




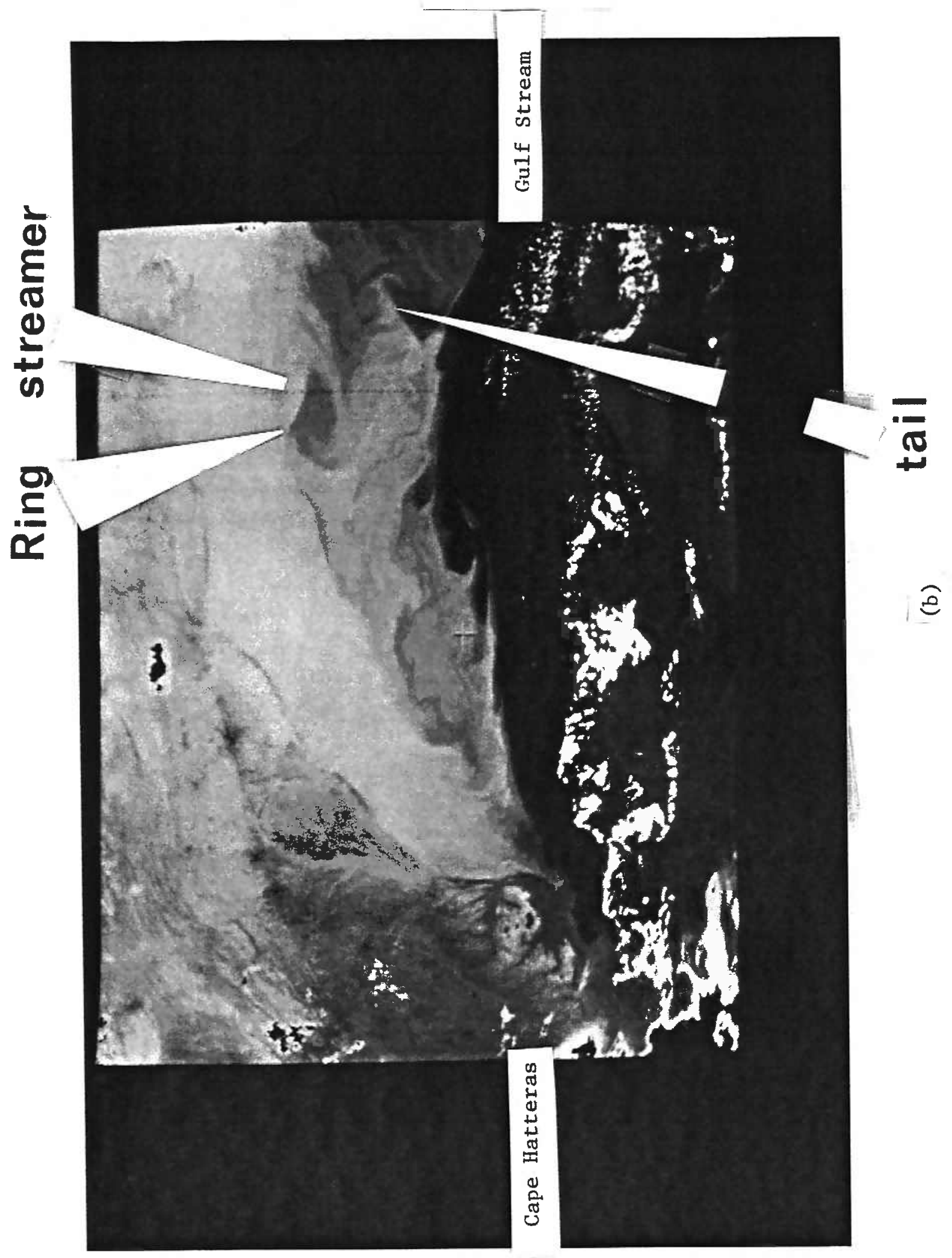




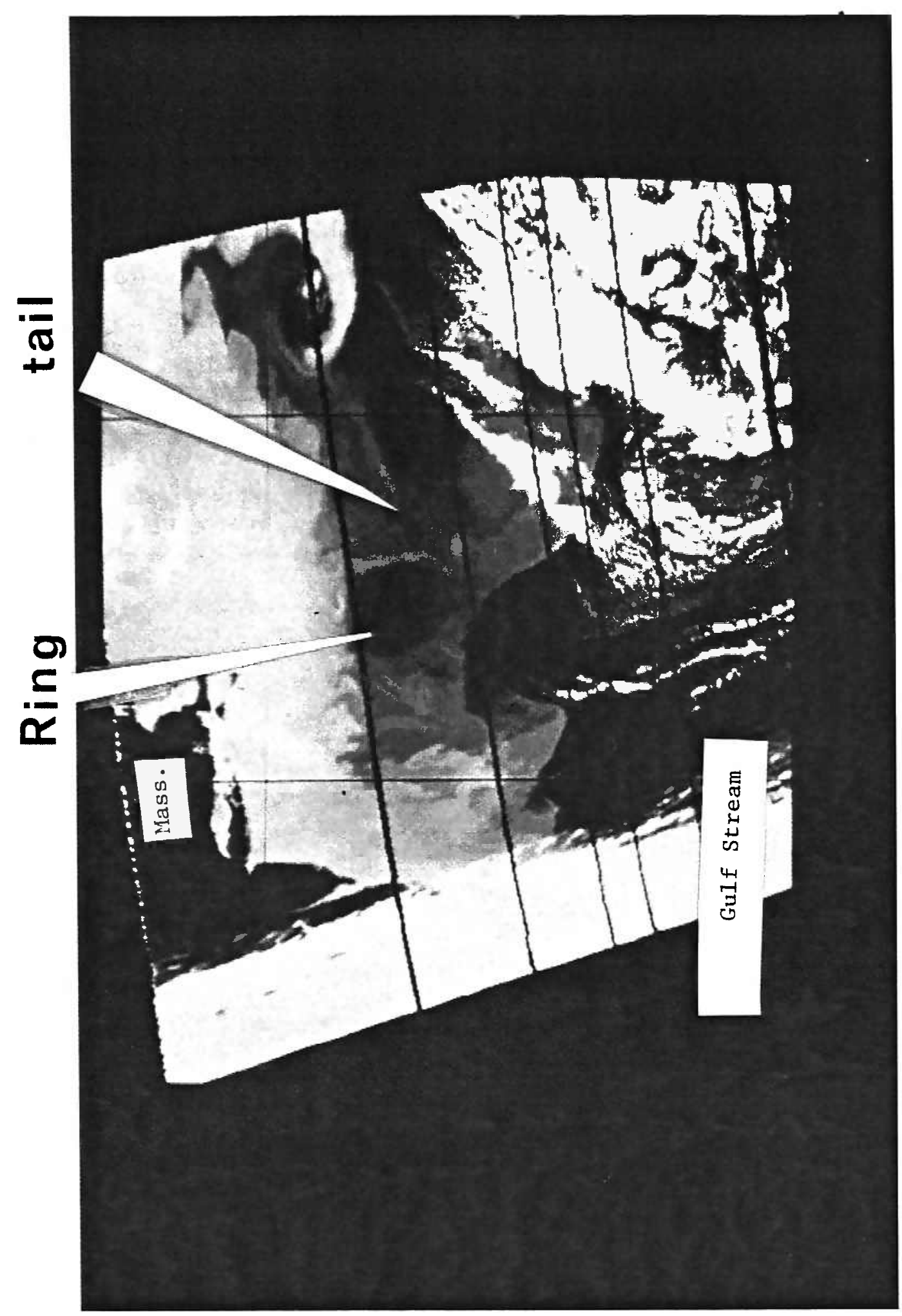


In $d$, we see evidence of a trailing low pressure center behind the Ring, where the streamer of Shelf Water has wound into a cyclonic swirl. Also, there is a suggestion of a broad northward flow ahead of the Ring. 


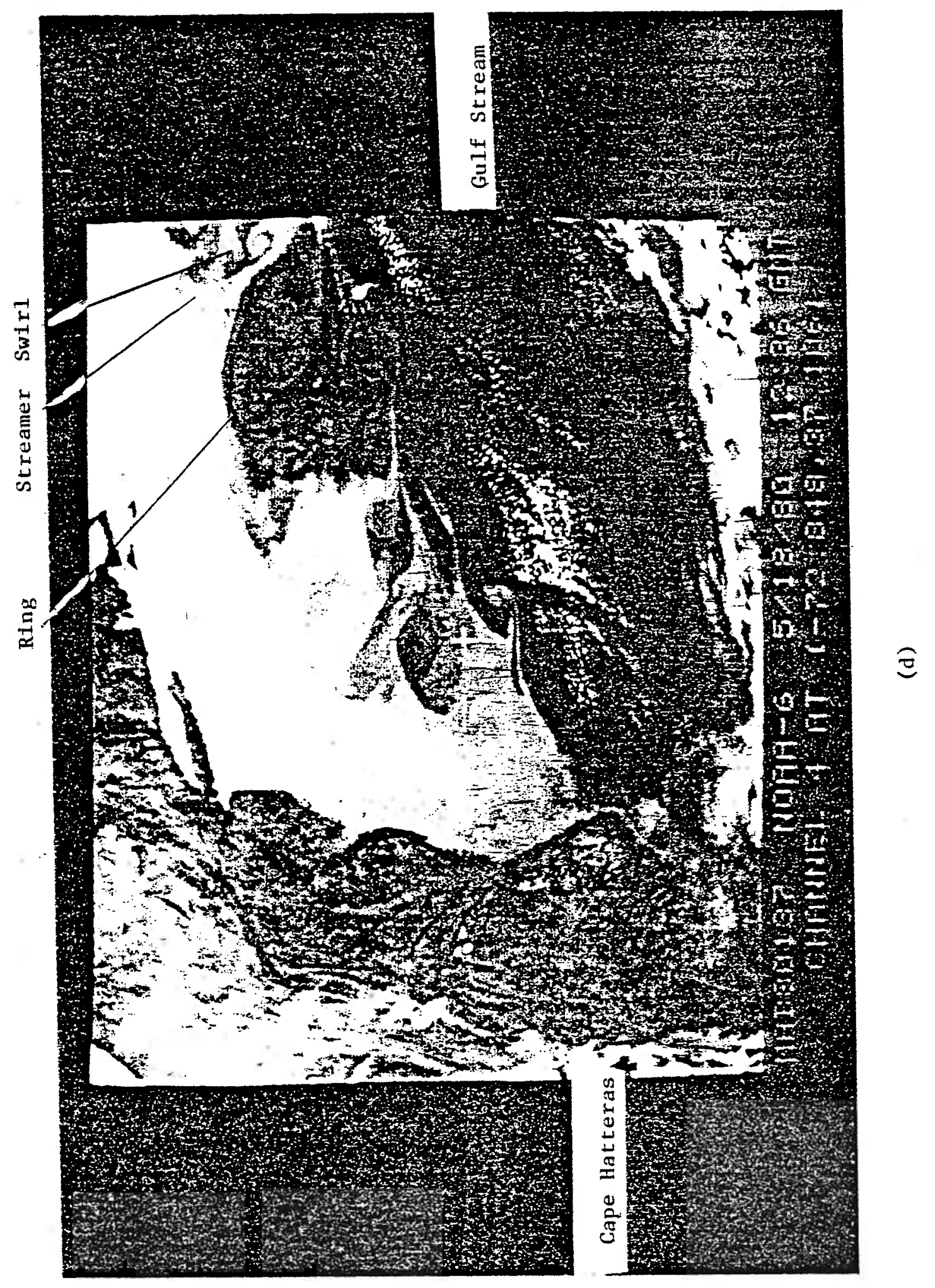


III.c Advection and Diffusion in a Dynamic Ring Model-

The results of the previous section immediately come into question when we start to investigate the dynamic consistency of the advection field. If $\psi$ is the horizontal structure function of a large scale flow, Its evolution should be governed by quasi-geostrophy, which requires that $\chi$ also be contours of potential vorticity. In Fig. III.7, we have included a plot of the potential vorticity, q, from McWilliams and Flierl's equivalent barotropic Gulf Stream Ring simulations. In this case, $q$ is defined by:

$$
q=\left(\nabla^{2}-r^{2}\right) \alpha+y /\left(Q \xi_{111}\right) \quad \text { Eq. III. } 8
$$

where $\alpha$ is the non-dimensional version of $\mathcal{Z}$. It is obvious that $q$ does not particularly resemble $\chi$, so it seems unlikely from a dynamic point of view that particles will flow along the streaklines of Fig. III.I, and therefore, it is not clear that we may employ the intuition gained from the model of the previous section. 1 The mismatch of $\mathcal{X}$ and $q$ is due to the fact that $\psi$ in Eq. III.5 is not a solution to the equations of motion. We will now infer the particle trajectories from McWilliams and Flierl's model Ring, which is dynamically consistent, and compare them with the kinematic model, as well as analyze particle motion in

1 There are several examples of dynamically consistent, steadily propagating, permanent-form solutions, for which the dual conservation by a particle of streakline and potential vorticity is automatically satisfied. For example, the equation:

$$
q=\left(\nabla 2-\gamma^{2}\right) \alpha+y /\left(Q S_{111}\right)=f(X)
$$

is recognized as the governing equation of the modon, or solitary eddy solution, of Flierl, Larichev, McWilliams, and Reznik (1980). These have very different streakline and potential vorticity patterns from those in Figs. III. 1 and 7. 


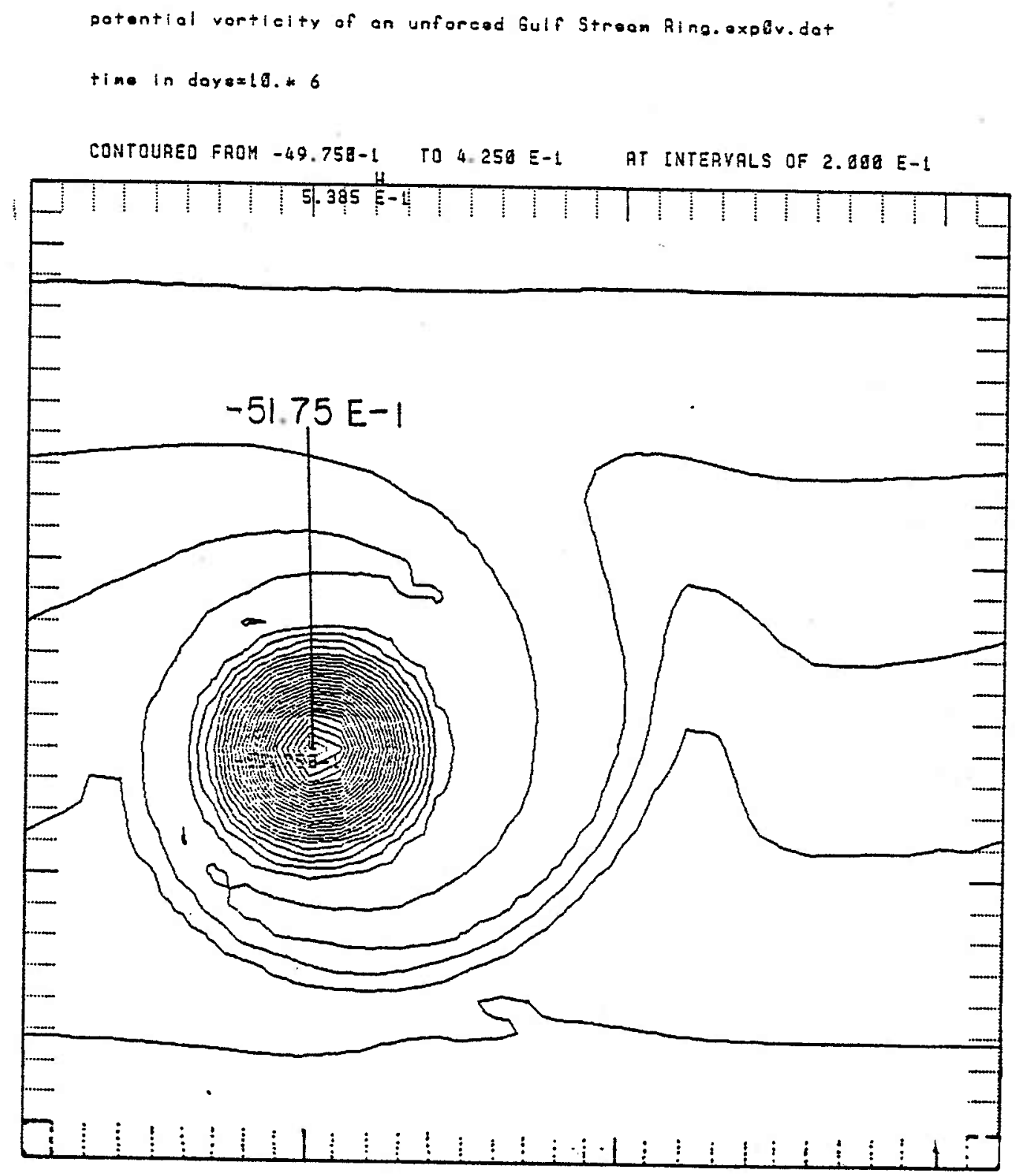

Figure III.7. Potential Vorticity

Here we plot $q$, the potential vorticity, defined by:

$$
q=\left(\nabla^{2}-r^{2}\right) \alpha+y /(Q \xi 111) \text {, }
$$

at day 60 , from McWilliams and Flierl's equivalent barotropic Ring. This q configuration was largely retained by the Ring throughout the experiment ( 160 days). 
terms of dynamics. The principle result will be that the general Lagrangian character as described in the previous section will be retained, but some of the details will be altered. Also, the importance of friction and wave dispersion will become apparent.

Dynamic 'Streaklines'-

In Fig. III.8, we have plotted $\alpha$ at various stages of evolution from McWilliams and Flierl's model Ring. Notice the azimuthally asymmetric field, consisting primarily of a single trailing low pressure and a single leading high pressure, which surrounds the coherent, large amplitude Ring. The quasi-steady propagation of the Ring (see Table III.2) and its nearly permanent form shape suggests that we compute streakfunctions for the Ring according to Eq. III.4, using the average propagation speed of Table III.2 for 'c'. In Fig. III.9, we plot such a $X$ at days 40,60 , and 80 from the streamfunctions in Fig. III.8. Note the persistent shape of $\chi$ over this period, even though 80 days represents roughly 8 eddy turn-over times. For reference, the corresponding streakfunction, as computed using 01son's empirical streamfunction, but with $\subseteq$ as determined by Table III.2, is also shown in Fig. III.9. For convenience, we shall refer to the streaklines as computed from Fig. III.8 as the dynamic streaklines, while those computed from Olson's streamfunction will be called the kinematic streaklines. A comparison of the two reveals both many gross features in common, e.g. the existence of a trapped zone, and several marked contrasts, e.g. the location of the stagnation point. 
(a)

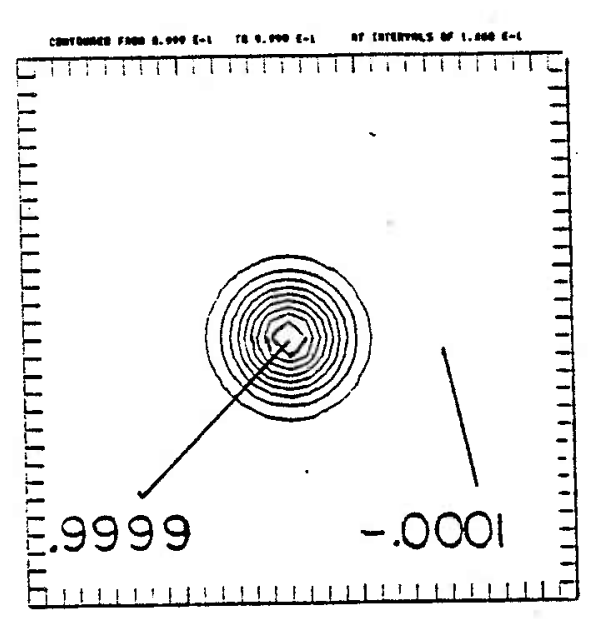

(b)

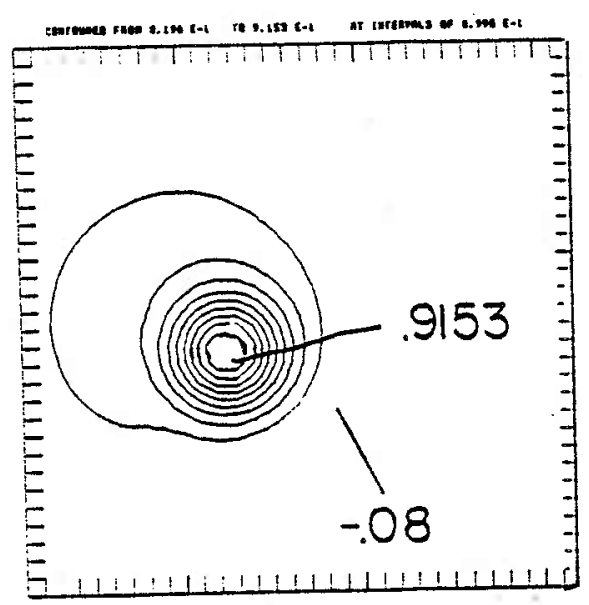

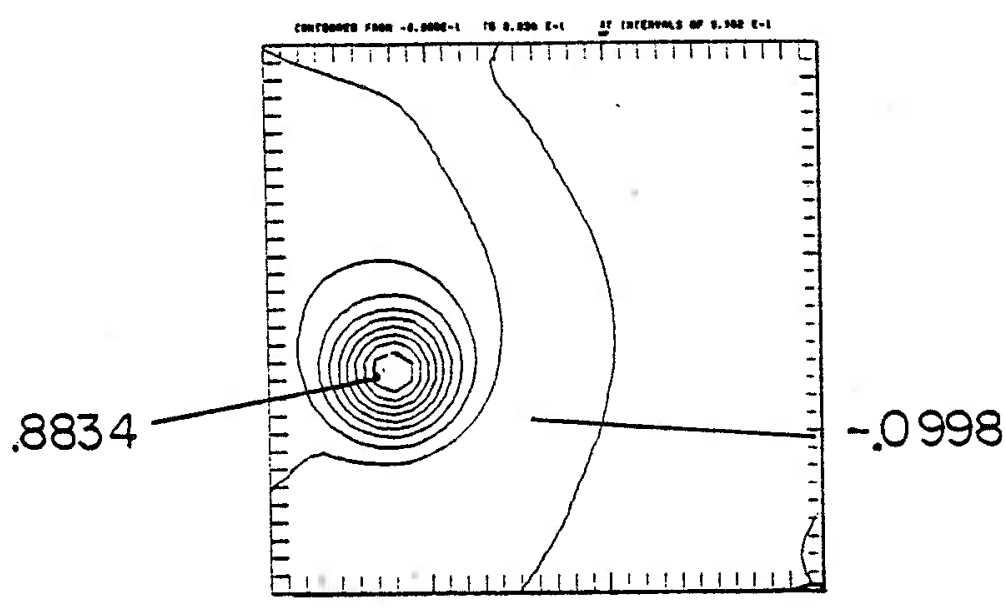

(c)

Figure III.8. Dynamic Ring Evolution

Here we plot the horizontal structure of the dynamically evolving Ring at days (a) 0 , (b) 40 , and (c) 80 . Note the development of the azimuthally dependent field, the Ring propagation, and the nearly permanent form evolution. The parameters in this experiment were, $K=5 \times 10^{-4}, \Gamma^{2=2}, \xi_{111=2.1}$, and $Q=4.76$. 
Measured Pattern Propagation Speeds

From McWilliams and Flierl's Equivalent Barotropic Model

\begin{tabular}{|c|c|c|c|c|}
\hline Day & & x Location & y Location & Velocity \\
\hline $\begin{array}{l}40 \\
45 \\
50 \\
55 \\
60 \\
65 \\
70 \\
75 \\
80 \\
85 \\
90 \\
95 \\
100\end{array}$ & 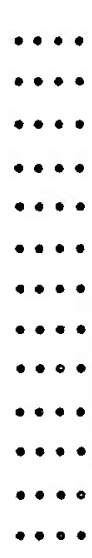 & 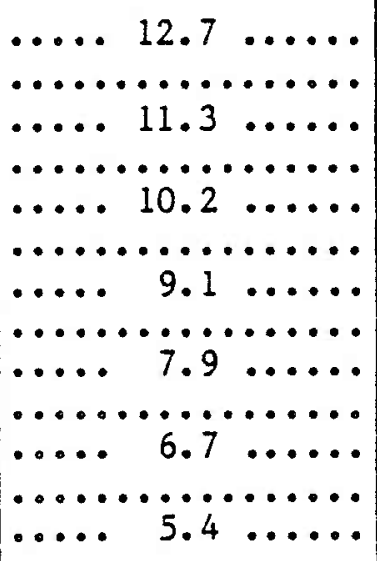 & 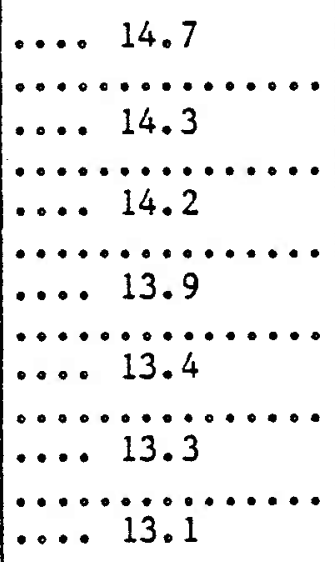 & $\begin{array}{l}(-2.7,-.7) \\
(-2.2,-.2) \\
(-2.2,-.7) \\
(-2.4,-.9) \\
(-2.4,-.2) \\
(-2.2,-.4)\end{array}$ \\
\hline
\end{tabular}

All locations are expressed in grid units $\left(\delta_{x}=\delta_{y}=20 \mathrm{~km}\right.$ dimensionally), and are from a $32 \times 32$ numerical experiment. The velocities are expressed in $\mathrm{km} /$ day. The error on position estimates is .2 of a grid, which translates to an error for the pattern speed of .4 $\mathrm{km} /$ day. The average velocity for the whole interval is $(-2.4,-.5)$ $\mathrm{km} /$ day, with an error of $.04 \mathrm{~km} /$ day, which is within the error associated with all of the above velocities. 
(a)
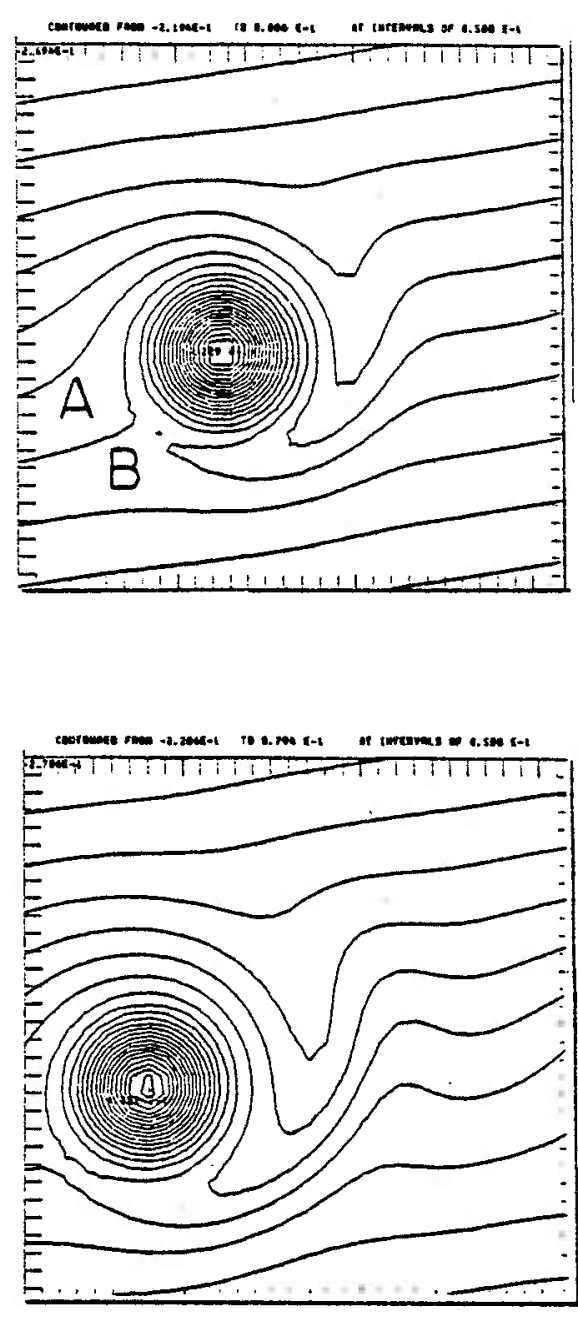

(c) (b)
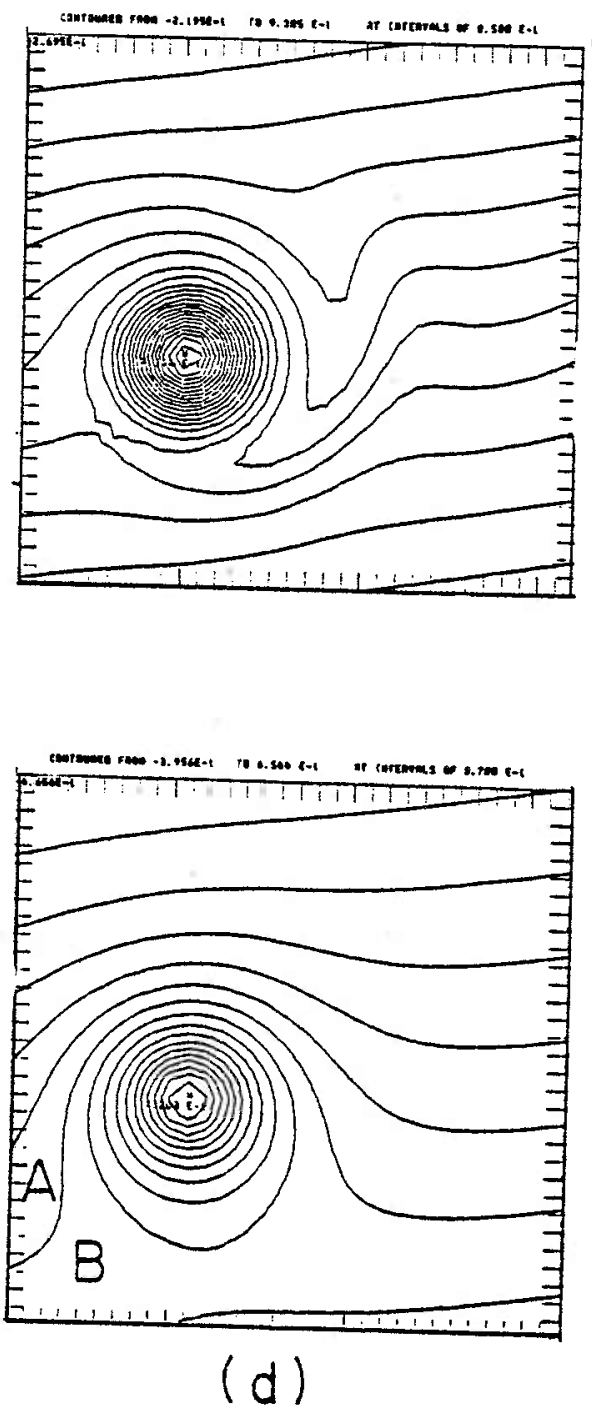

Figure III.9. Dynamic Streakfunctions

Here we have plotted the dynamic streakfunctions appropriate to days (a) 40 , (b) 60 , and (c) 80 , as computed by an application of the kinematic formula to the dynamic Ring, and using $c=(-2.4 \mathrm{~km} / \mathrm{day},-.5$ $\mathrm{km} /$ day). Notice the nearly permanent form of the Ring. For comparison, in (d) we have included the kinematic streakfunction as computed from olson's streamfunction, with $c$ as above. 
Perhaps the most apparent difference between the sets of contours in Fig. III.9 is that for the dynamic streakfunctions, the contours ahead of the Ring are no longer mirror images of those behind it. Notice, for example, that the stagnation point in Figs. III.9.a, b, and $c$ has been rotated clockwise with respect to the location of the same in Fig. III.9.d. Also, the dynamic streaklines north of the Ring, are pulled abruptly to the south upon crossing to the east of the Ring, which is in contrast to their rather gradual rise over the Ring from the west. Secondly, we notice that the critical streakline originates to the west of the Ring, rather than to the southwest, as in Fig. III.9.d. Therefore, a particle initially at point ' $A$ ' in the dynamic streaklines would follow a slightly warped version of that path it would take in the kinematic model, while a particle initially at 'B' would circumnavigate the Ring to the south, in contrast to that path it would take in the kinematic Ring.

The accuracy of these streaklines will be addressed shortly. Taken as correct, they imply that the fluid which ultimately reaches the southeastern side of the stagnation point originates to the west, which is a feature observed in satellite photographs. Also, note the asymmetry of the streaklines, which implies that more of the fluid passing anticyclonically around the Ring is bought closer to the trapped zone than in the kinematic model. Proximity to the Ring allows for fluid to alter the trapped zone water properties, therefore, relative to the kinematic model, a larger area of exterior fluid in the dynamic model can exchange with the trapped zone (see Fig. III.10). To test this idea, we performed a pair of advection-diffusion experiments in 

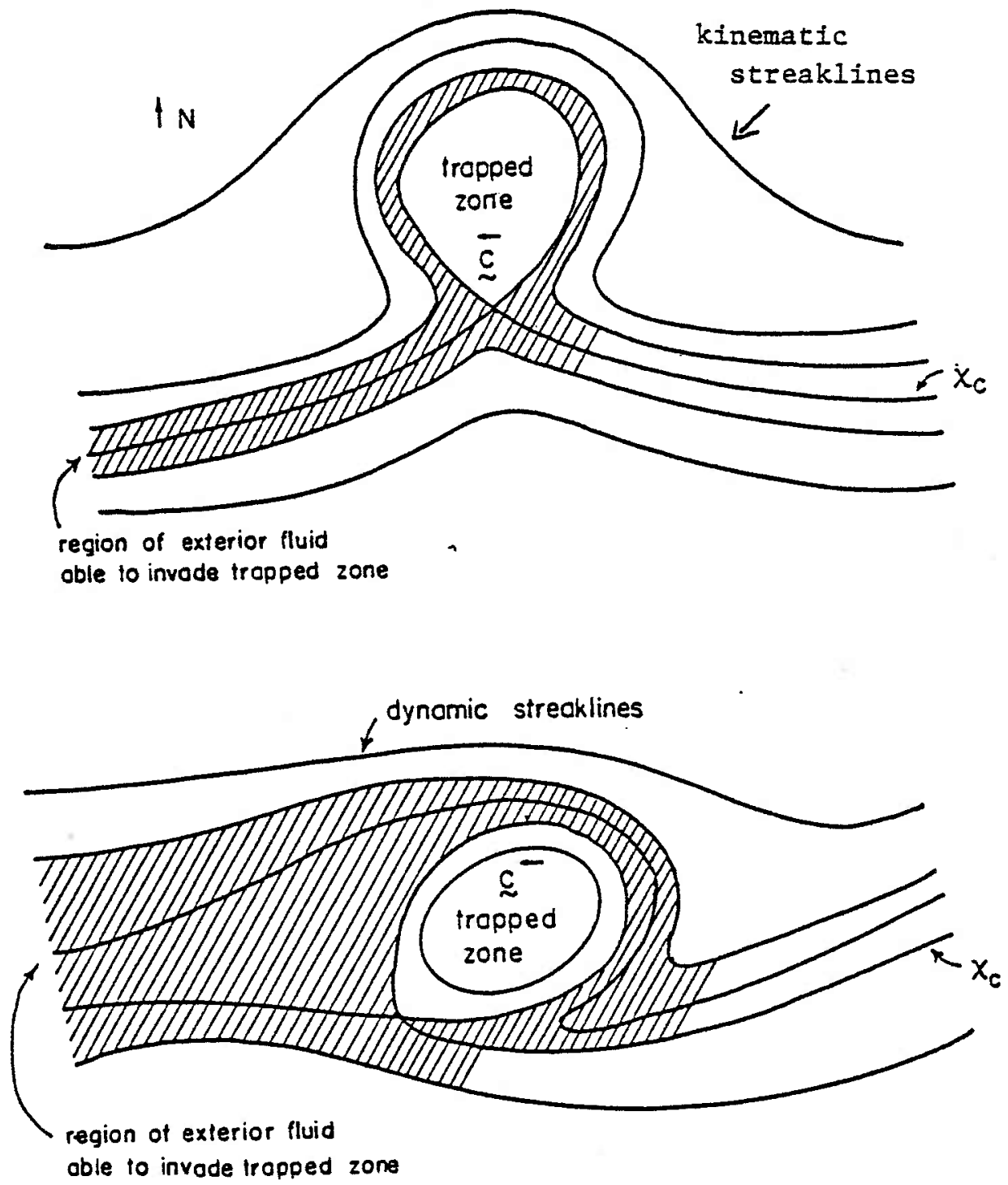

Figure III.10. An Implication of Asymmetric Streaklines

The area of fluid able to invade the trapped zone in the dynamic Ring is increased relative to the same for the kinematic Ring due to the asymetry of the streaklines. 
which an initial spike of marked fluid was situated to the west of the Ring, near the northern latitude of maximum Ring velocities. After 70 days, it was found that a greater amount of the marked fluid had entered the dynamic Ring trapped zone, compared to that for the kinematic Ring: While this simulation corroborated the basic idea about the larger area for the origins of entrained fluid, recall that 1 is a result which depends on $\mathrm{K}$ and on the Fickian closure.

Finally, the dynamic Ring velocity field is oriented so that the area of the trapped zone is roughly $75 \%( \pm 5 \%)$ of that in the kinematic model. Although the radil of maximum velocities are matched in these two Xfields, the exterior velocity field of the dynamic Ring decays much more rapidly away from the Ring.

The Importance of the Dispersion Field-

As pointed out by McWilliams and Flierl, the pressure field neighboring the Ring in Fig. III.8 consists principally of leading high and trailing low centers, which are initially set up by dispersion. Consider the kinematic effect of a trailing low on an otherwise purely westward moving, radially symmetric pattern (see Fig. III.11). The trailing low has a cyclonic flow, which augments the azimuthal velocity between the Ring and the low center, and weakens it otherwise. In the steadily-propagating frame of the Ring is a stagnation point, where:

$$
\begin{array}{ll}
c=u_{\text {ring }}+u_{\text {low }} & \text { Eq. III.9 } \\
0=v_{\text {ring }}+v_{\text {low }} & \text { Eq. III. } 10
\end{array}
$$

If $\underline{u}_{10 w}=0$, the stagnation point will occur on the line $x=0$, 


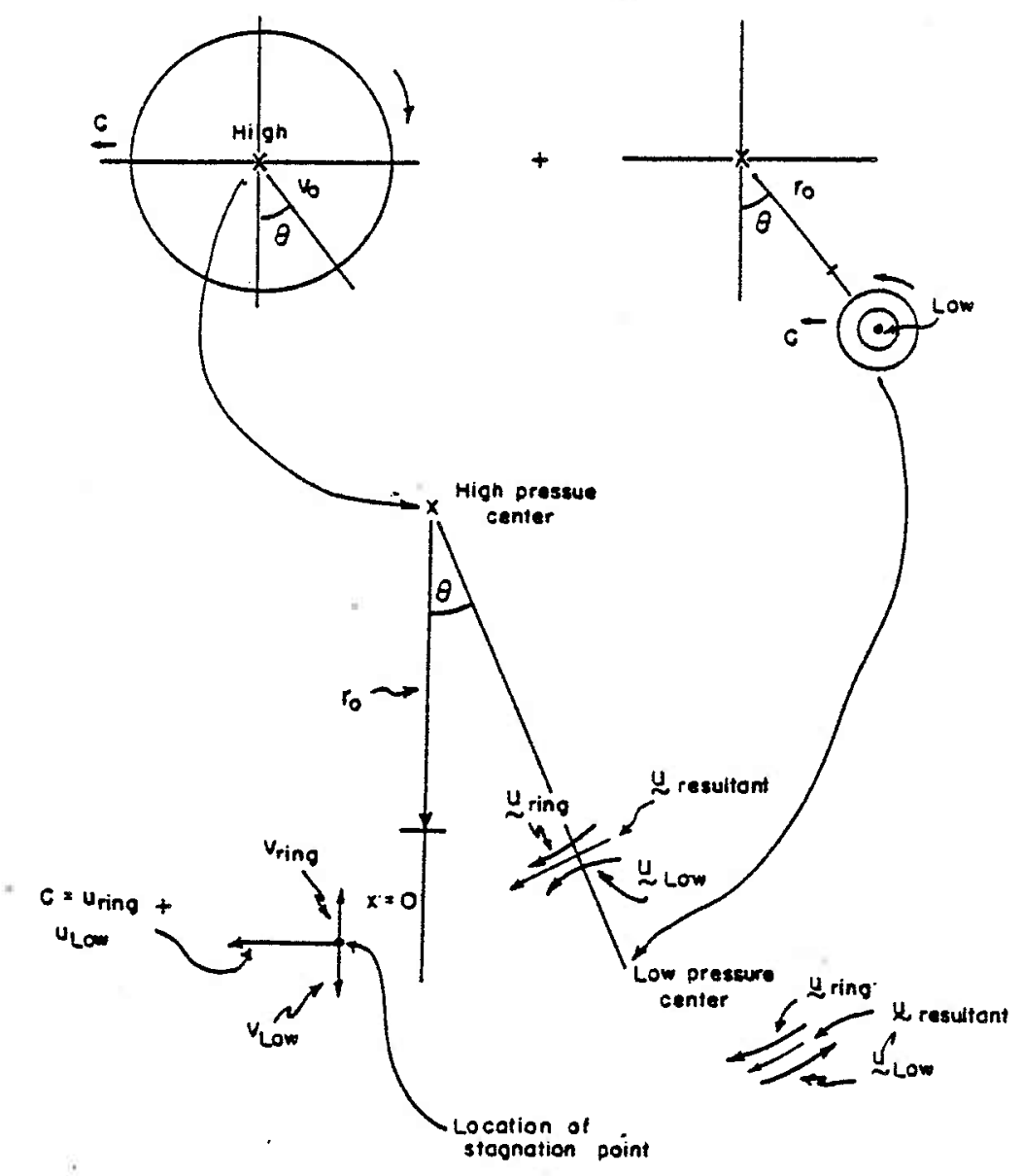

Figure III.11. The Advective Effect of a Trailing Low Pressure

Near to the Ring, the trailing low intensifies the anticyclonic Ring velocities, which effectively 'pushes' the stagnation point to the west. 
which intersects Ring center. If, however, $\underline{u}_{10 w}=0$, west of the low, $v_{\text {low }}$ is negative, requiring the stagnation point, by Eq. III.10, to be west of the line $x=0$. The advection by the low pushes the stagnation point in the direction of Ring motion, which explains the position of the stagnation point in Fig. III.9.

Now consider the analogous effects of the leading high pressure center. West of the Ring, the center produces an anticyclonic flow, which weakens the northward directed azimuthal flow near the Ring, but strengthens it farther away. Fluid parcels west of the Ring encounter the leading high before the trapped zone arrives, and they gain a slow northward motion, resulting in the gentle rise of the streaklines from the west. The fact that the Ring is subcritical with respect to the fastest avallable Rossby wave speeds allows the front to precede the Ring and produce a non-negligible 'upstream influence'.

Hence, the lowest order shape of the streakline field is strongly influenced by the advective effects of the neighboring high and low pressure centers. The existence of these centers and their motion relative to the Ring are the consequences of the governing dynamical equation, and are the structures missing from the kinematic model necessary to make its particle paths dynamically consistent.

Advection-Diffusion Using Dynamical Advection Fields-

All results regarding the streakline field assume that it accurately describes particle trajectories, although strictly speaking 
streaklines are only appropriate for a steady state structure and the Ring is evolving in time. Now we will verify the Lagrangian field through a series of advection-diffusion experiments.

In the kinematic models, there were two very different regions of Lagrangian flow; a trapped zone associated with the high velocity core, and an exterior, excluded from the trapped zone by a closed streakline. Presently, we shall examine the dynamic Ring for a trapped zone, and then the exterior for its particle trajectories.

In Fig. III.12, we plot tracer concentration as determined by Eq. III.7, where the advection field was provided by the streamfunction in Fig. III.8. In this experiment, the initial tracer distribution was Gaussian with a length scale of $60 \mathrm{~km}$, and was situated in the Ring. The initial tendency for the tracer is to spread radially; however, upon filling a certain area, which propagates with the Ring, the radial spread slows to a halt. Subsequently, the tracer exits via a rather thin tail attached to the Ring southern edge. The fluid velocities in the vicinity of point 'A' of Fig. III.12 are roughly the same as the the pattern propagation velocity. This was checked by computing the Eulerian velocity at ' $A$ ' and comparing it to the propagation velocity associated with the motion of the maximum amplitude in the streamfunction; both were $0(-2.4 \mathrm{~cm} / \mathrm{sec},-.5 \mathrm{~cm} / \mathrm{sec})$. On the basis of this simulation, we infer that the trapped zone predicted by Fig. III. 9 exists. Note also that the area of the fluid moving with the Ring in Fig. III. 12 agrees with the trapped zone area of Fig. III.9. Hence, the interior streaklines of Fig. III.9 provide a reasonable picture of 
(a)
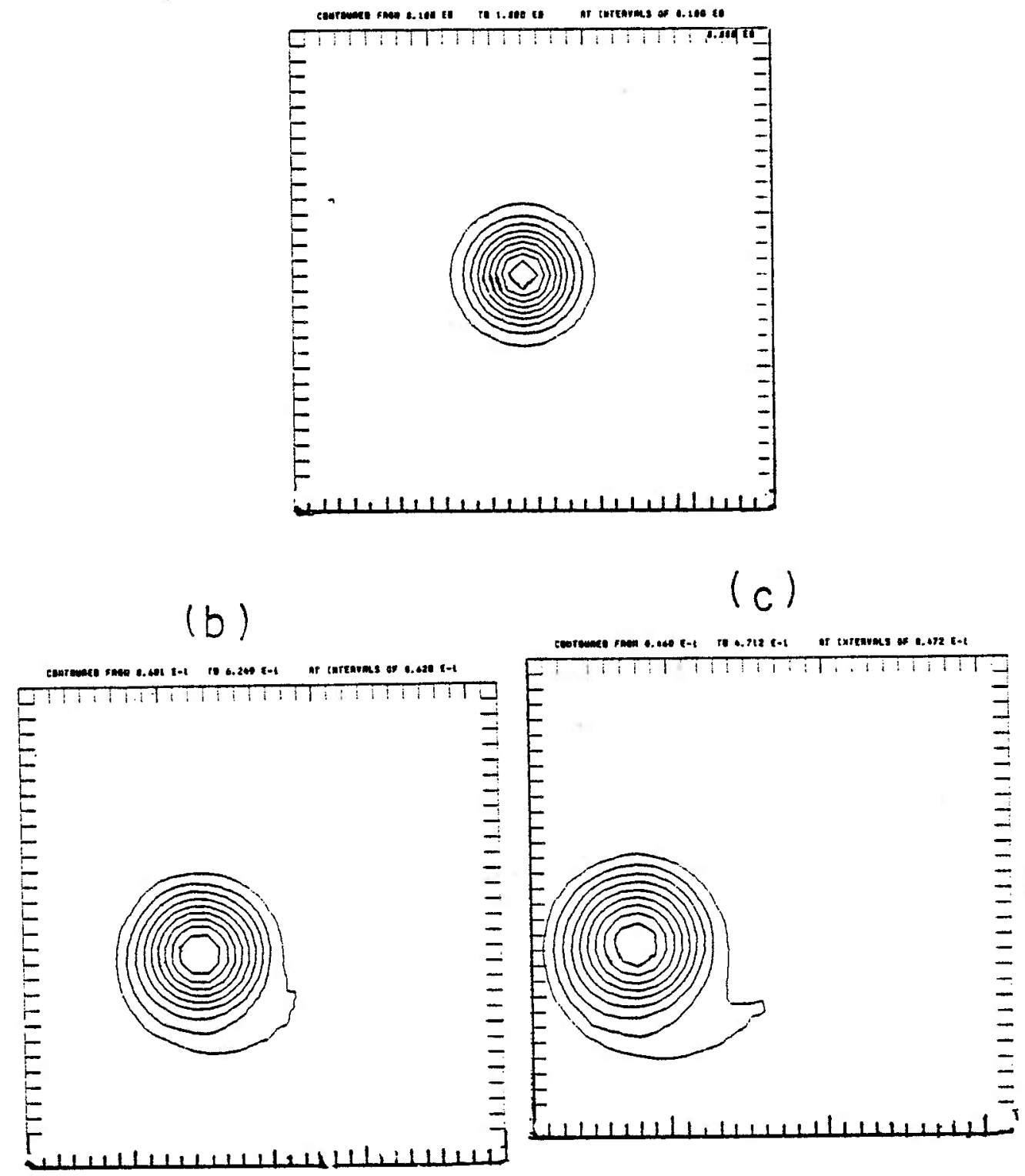

Figure III.12. Advection-Diffusion by the Dynamic Ring

Here we plot days (a) 0 , (b) 40 , and (c) 80 , from a numerical solution to Eq. III.7 using the dynamic Ring velocity field. Note the tail formation and the trapped zone of fluid. 
particle motion in that region. As an aside, note that the fluid velocities of the area where the interior fluid exits the trapped zone match the propagation velocity of the Ring, as in the kinematic model results. Therefore, the dynamic Ring model also predicts that we should observe tracer exchange in Rings to occur near the stagnation point, or for warm (cold) Rings, to the south (north) of the hydrographic Ring signature.

Critical Contour-

Recall that the critical contour, $\chi_{c}$, divides the exterior fluid into two regons, one of which circumvents the trapped zone anticyclonically, and the other cyclonically. In Fig. III.13, we present the results of an experiment designed to test the location of $\chi_{c}$. An initial Gaussian of grid point scale was placed at a point on the critical streakline predicted by Fig. III.9 (grid point location $(5,9)$ ) and its subsequent interaction with the Ring was computed. It is clear in these figures that about half of the marked fluid moves north about the Ring, and about half south, which supports the location of $\chi_{c}$ in Fig. III.9.

Exterior Streaklines-

We can accurately compute solutions to Eq. III.7; therefore, to test the exterior streaklines, we have conducted pairs of advection- 

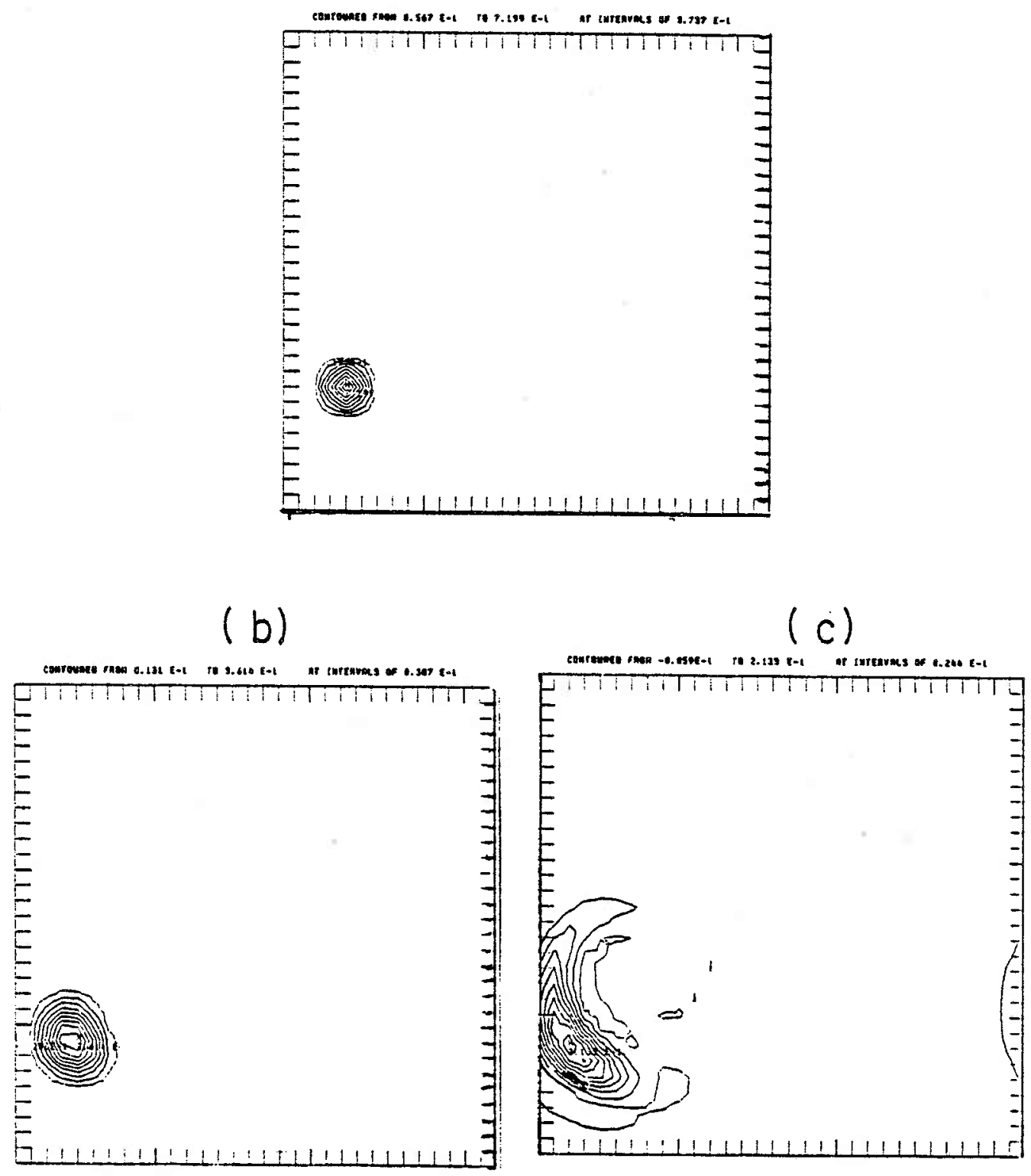

Figure III.13. Critical Contour

In this experiment, we introduced a blob of fluid on the predicted critical contour. Notice that the fluid splits, with roughly one half moving around the trapped zone in either direction indicative of behavior near critical contours. Shown are days (a) 0, (b) 20, and (c) 50. 
diffusion experiments and inferred particle trajectories from them. 2 Of the two members in each experiment set, one member employed the time-dependent velocity field of the dynamic Ring, and the other used the steadily propagating velocity field contained in Fig. III.9.a, 1.e.:

$$
\alpha\left(x-c_{x}(t-40 \text { days }), y-c y(t-40 \text { days }), 40 \text { days }\right) .
$$

In each experiment set, both members of the pair used the same initial condition, which was a grid-scale Gaussian spike of marked fluid. Different experiment sets were characterized by different locations of the initial condition, and all the sets together tested the fifth column of grid points from rows 7 to 15 .

In Fig. III.14, we compare the results of the pair with initial conditions at $(5,15)$. This location put the marked fluid in a region of strong shear (recall Fig. III.2). In Fig. III.14.a and b, we plot the S field at day 40 from which we note that the fields compare visually quite well. (This degree of similarity was typical for all experiment pairs, and for the duration of each.) In $c$, we show the result of a similar experiment using the symmetric field proposed by 01son. From this comparison, it is clear that the asymmetric streaklines are better approximations to the flow field than the symmetric field. Measurements from $a$ and $b$ were also encouraging. The maximum value of the tracer and

2 Haidvogel (personal communication) has demonstrated that the particle trajectories computed using Eqs. II. 2 are such that the constraint of $q$ conservation is not satisfied; therefore, we have opted not to employ that technique to compute particle trajectories. 

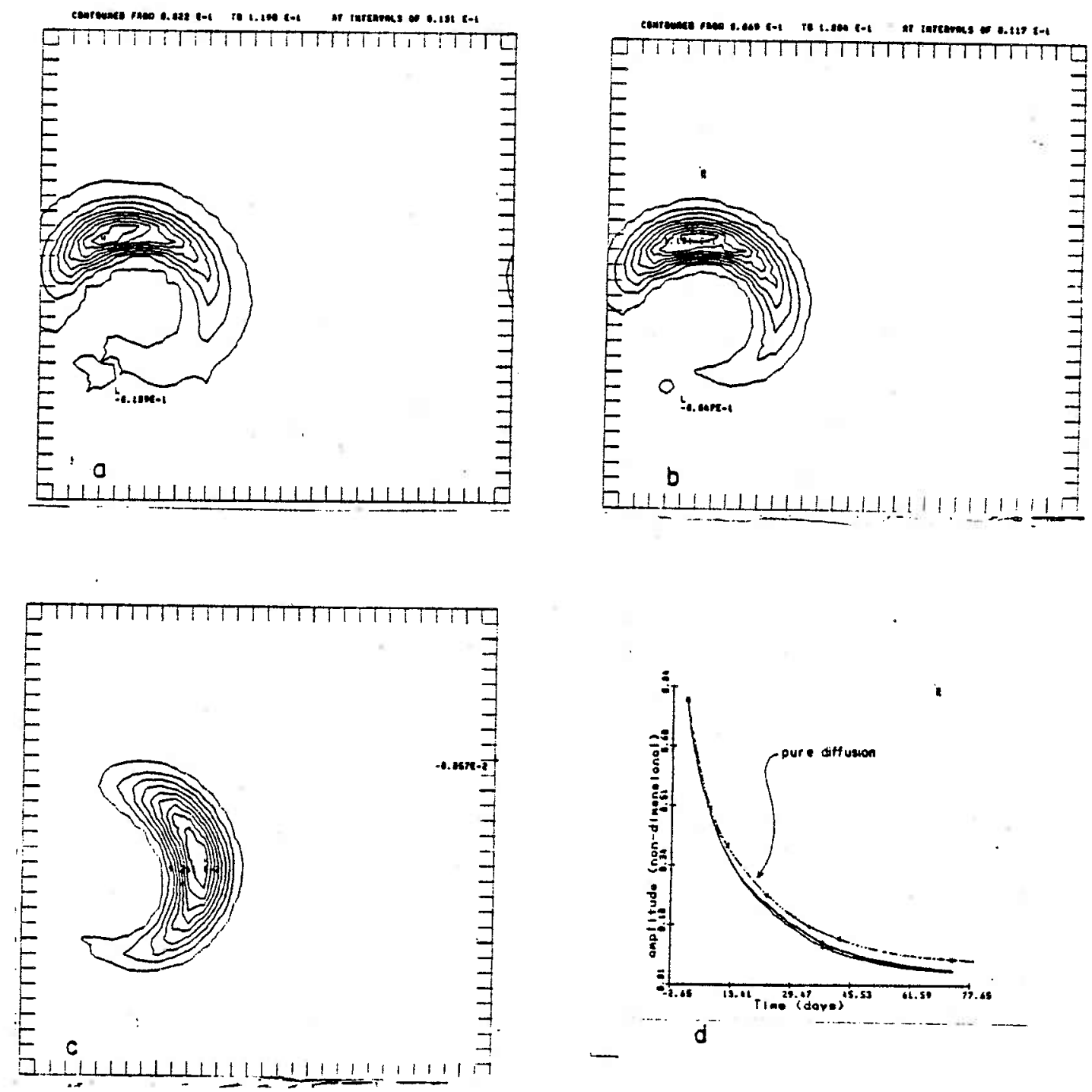

Figure III. 14. The Exterior Streaklines

We compare the results of a pair of experiments initialized with a marked blob at grid point location $(5,15)$, which is located in a region of high shear. The field in (a) used the evolving Ring, and (b) the dynamic streaklines. Visually, the fields agree well. In (c), we show the results of the same experiment using (d) from Fig. III.9. Obviously, the dynamic streaklines are a better representation of the flow of the Ring. In (d), we compare the history of the amplitude maximum from the experiment pair. Also, we plot Smax as determined by pure diffusion. It is evident that the solutions are quickly affected by shear. 
1ts motion are dependent on velocity and shear, 3 and were used to test the agreement more of $a$ and $b$ more quantitatively. In $d$, we compare the values of $S_{\max }$ for the experiment in $a$ and $b$. Note we have included $a$ history of Smax as determined by simple diffusion, which indicates that the Ring solutions are affected by the shear. The agreement in this diagram is representative of all the pairs. Finally, with respect to the motion of $S_{\max }$, this experiment turned out to have the worst comparison between the pairs. Even so, the average error in velocity was roughly $.3 \mathrm{~cm} / \mathrm{sec}$, as compared to the average velocity of $3 \mathrm{~cm} / \mathrm{sec}$. All other comparisons of the motion of Smax were better than this.

On the basis of these comparisons, we conclude that the steadily propagating field accurately estimates the shear and velocity of the evolving Ring outside of the trapped zone. Therefore, we conclude that the streaklines associated with the steadily-propagating field are an accurate representation of the particle trajectories of the dynamic Ring.

In summary, having tested the critical contour, trapped zone, and exterior streaklines of Fig. III.9, and shown them to accurately describe the Ring particle flowfield, we conclude that although the Ring is evolving in time, its variability does not strongly alter its Lagrangian patterns from those of a steadily-propagating structure.

3

The maximum concentration of dispersant is sensitive to strong shear (recall Fig. III.2). For example, a Gaussian in a linear velocity profile eventually decays as $t^{-3 / 2}$, as compared to $t^{-1 / 2}$ for simple diffusion. Using the scales appropriate to a Ring, we estimate that the effects of the velocity field will dominate diffusion after 10 days. 


\section{III.d Potential Vorticity Considerations-}

In contrast to the assumed pressure field of section $b$, $\alpha$ in Fig. III.8 was determined by integrations of an equation of motion, Eq. II.14 (the present form of the equivalent barotropic equation):

$$
\left(J^{2}-r^{2}\right) \alpha_{t}+Q \sum_{111} J\left(\alpha,\left(v^{2}-r^{2}\right) \alpha\right)+\alpha x=K_{v} 6_{\alpha}, \quad \text { Eq. II. } 14
$$

which states that along a fluid path, potential vorticity, defined by Eq. III.8, is altered only by non-conservative processes. Consider now the comparisons of $q$ and the dynamic $\chi$.

In Fig. III.7, we show a contour plot of potential vorticity at day 60 of the dynamic Ring calculations, the most noticeable feature of which is the large negative potential vorticity zone located at Ring center. For a Ring, this pool would find its origins to the south of the Gulf Stream, having moved northward during formation. The second thing to note in Fig. III.7 is the strongly perturbed q contours located just outside the pool, which look as if they have been wrapped around the Ring. Once set up, this shape of the exterior potential vorticity field was maintained for the duration of the experiments.

Comparing the potential vorticity (q) of the Ring interior with the tracer experiments in Fig. III.12, we see that the trapped region of tracer corresponds closely with the region of anomalously negative potential vorticity. Recall, the only mechanism which will alter the potential vorticity of a fluid parcel is dissipation, which in these experiments is very weak although it does largely account for the change in trapped zone q. Given the slow evolution of the $q$ distribution, we 
conclude that the fluid within the Ring core largely retains its original composition, there being no sources for potential vorticity of sufficient strength to allow for much exchange between trapped zone and exterior.

In the kinematic models of section $b$, we demonstrated that particle trapping was a consequence of large Q. Strong nonlinearity, in conjunction with coherent flow, has the same effect dynamically. To see this, consider perturbation expansions of the potential vorticity, $q$, and the streamfunction, $\chi$, in powers of $1 / Q$. To lowest order, the potential vorticity becomes:

$$
q_{0}=\left(\nu^{2}-1+2\right) \alpha_{0}+O(1 / Q) \quad \text { Eq. III. } 11
$$

which states that within the Ring, the contributions to the potential vorticity by beta are negligible. Similarly, the lowest order dynamical balance from Eq. II. 14 is:

$$
J\left(\alpha_{0},\left(\sigma^{2}-r^{2}\right) \alpha_{0}\right)=0(1 / Q), \quad \text { Eq. III. } 12
$$

demonstrating a functional relationship between the lowest order potential vorticity and $\alpha_{0}$. However, $\chi$ to the lowest order in $1 / Q$ is:

$$
X_{0}=\alpha_{0}+c y / Q=\alpha_{0}+0(1 / Q), \quad \text { Eq. III. } 13
$$

which states that for large $Q$ the streaklines match the pressure contours; Eq. III. 12 is the condition that fluid parcels conserve potential vorticlty along streaklines. Hence, we can interpret particle trapping as a dynamical consequence of strongly nonlinear, coherent flow. In Fig. III.15, we plot the potential vorticity from Fig. III.8 against the baroclinic amplitude to demonstrate that Eq. III.11, valid 
Figure III.15. The Functional Relationship of $q$ and Streamfunction

Potential vorticity is plotted against streamfunction as determined by the dynamical Ring at day 40 . The range $.2<<1$ characterizes the Ring. The dashed line is a plot of the initial condition. Note that $q$ at small has been randomized, and there is some evidence of $q$ dissipation inside the Ring.

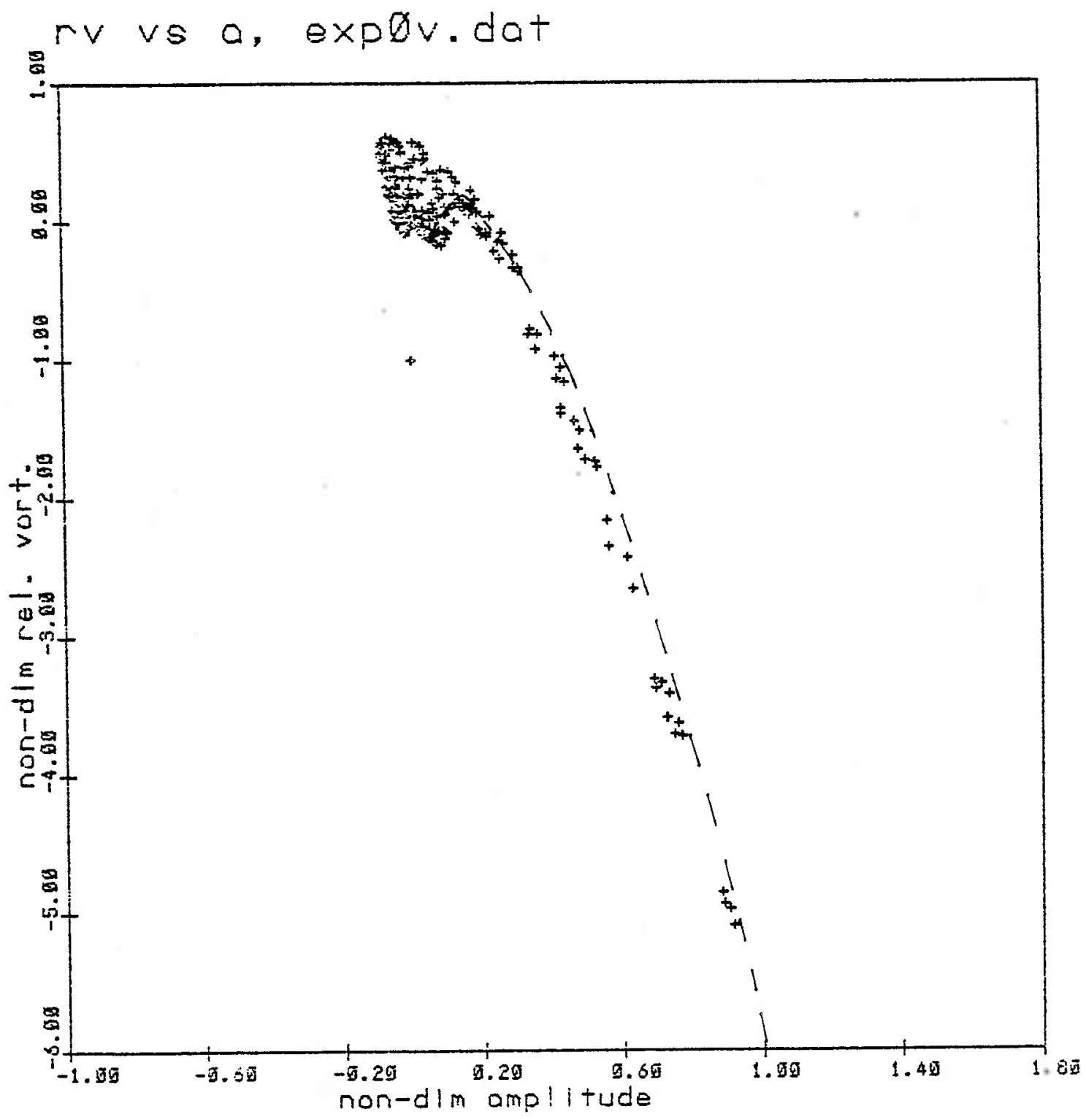


asymptotically in the limit of large steepness, applies to the present calculations where $Q \approx 0(5)$. In the range $.2<\alpha<1$, characteristic of the 'Ring' (cf. Fig. III.8), the fluid has a strong correlation between $q$ and $\alpha_{0}$

Ring Exterior-

Outside of the Ring, the velocities are no longer properly scaled by the maximum Ring velocities; therefore, the asymptotic expansion in the inverse of the steepness breaks down, and Eq. III.23 no Ionger is the lowest order dynamical statement. An inspection of Figs. III. 7 and 9.b shows that outside of the trapped zone, the streamfunction and potential vorticity are not functionally related. The place where the disagreement between these two fields is most obvious is near the trapped zone, where the potential vorticity contours look as if they have been wrapped around the Ring. In the far field of the Ring, $q$ contours are essentially determined by beta and oriented east-west and also disagree with the dynamic $X$, which have a slight southward slope. As the Ring is approached from either the east or west, the $q$ contours are warped by the dispersion centers, and align well with $X$.

The discrepancy between $q$ and $\chi$ in the far field is due to the limited lateral influence of the Ring. In the far field, the fluid parcels are not yet affected by the Ring, therefore the $\chi$ do not apply, and the particles move along $q$ contours. This is in contrast to very near the trapped zone, where the particles are under the influence of the Ring and move on the dynamic streaklines. It is clear from the 
potential vorticity contours at the southwestern corner of the Ring that fluid parcels are crossing lines of constant $q$, for as the Ring is approached from the west, those contours first turn north, and then back to the south. The Ring velocities, and therefore the fluid trajectories, are directed northward at that spot, from which we conclude that the fluid parcels are altering their potential vorticity. This can only be a result of dissipation. Indeed the potential vorticity structure near the trapped zone is near to the grid point scale, and thus a region of enhanced viscosity. Still, viscosity is relatively weak in its effects in comparison to advection, or to planetary vorticity, and the fluid reflects this in the particle paths circumnavigating the Ring. The gradient of $q$ about the $R$ ing is minimized azimuthally; therefore the streaklines depicted in Fig. III.9, which effectively predict particle flow in the direction of minimum $q$ gradients, are consistent with strong advection and weak potential vorticity loss.

Even though dissipation is having some effect, the overall potential vorticity configuration is not sensitive to $k$. In a run with smaller $k$, the only change in the $q$ contours was confined to within $a$ few grid points of the trapped zone boundary, where the contours were observed to 'wrap' further around the trapped zone than in Fig. III.7.

\section{III.e Implications-}

One of the more oceanographically interesting properties of Rings is that they transport water from the Slope Water to the Sargasso Sea. 
From this chapter, we have a dynamically sound understanding of this phenomenon, and a believable picture of the way fluid moves about the Ring. The important question with respect to the effects of Rings on their surroundings concern the magnitude of the exchange between the trapped zone and the exterior.

With regards to this problem, the results of this chapter suggest that tracer flux rates are small, because the constraint of $q$ conservation largely prevents fluid from entering or leaving the Ring. We have declined to make quantitative estimates of the flux, due to the crude parametrization of viscosity and diffusion. This chapter does suggest that an important effect of Rings is to act as a moving source of tracer, distributing quantities like potential vorticity, salt, and heat to the external fluid along a path which can extend far into the host regions. A separate Ring-induced tracer flux which we only begun to investigate here is that due to the production of streamers.

Finally, we have demonstrated that the dynamical particle trajectories are asymmetric with respect to east-west. For example, compare the critical streaklines in Figs. III.9. $X_{c}$ in the dynamic Ring model is located to the north of the same in the kinematic model. The immediate application of this result is to the interpretation of the streamers which are noted in thermal images of the sea surface in the Slope Water. According to the dynamic streaklines, the water which gets to the southeastern corner of the Ring, as streamers do, is located west of the Ring, rather than to the south as in the kinematic streaklines; hence, the dynamic streaklines are in better agreement with the 


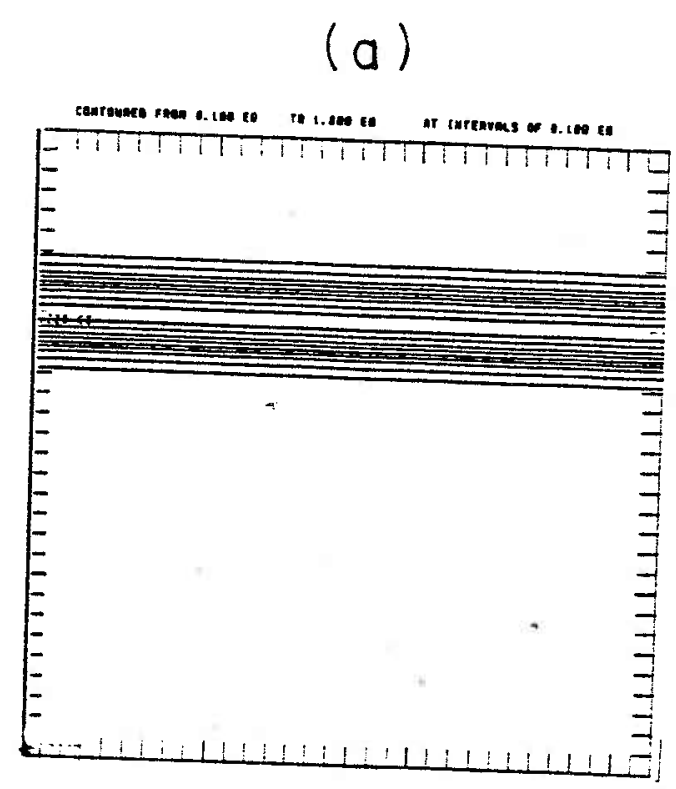

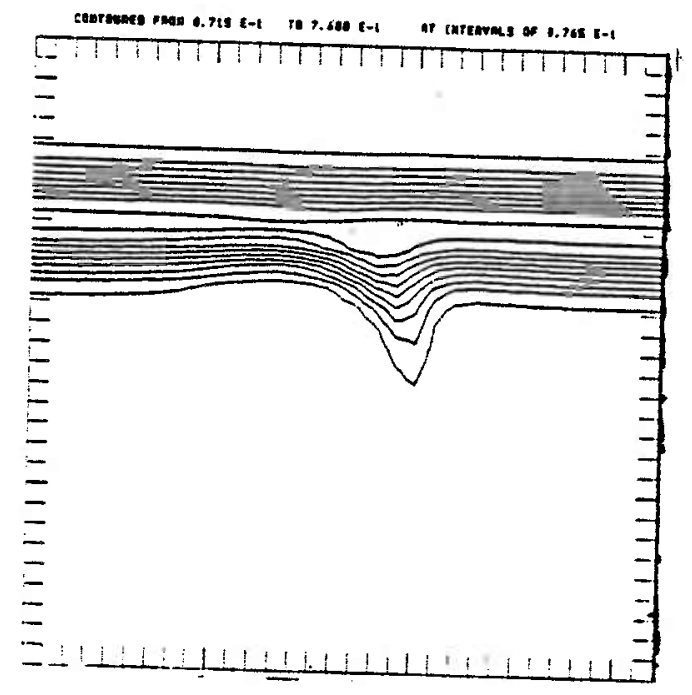

(b)

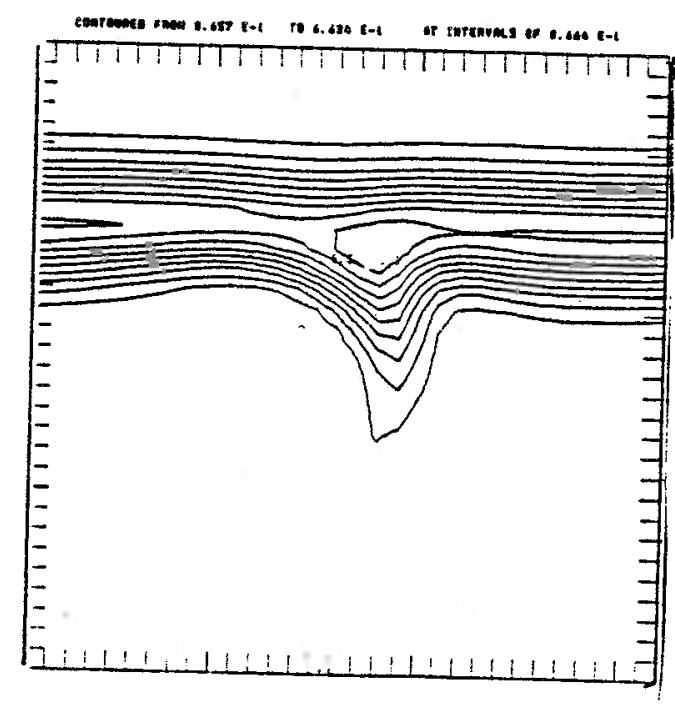

(c)

Figure III.16. Ring/Shelf-Slope Front Interaction

P lotted are days (a) 0 , (b) 20 , and (c) 40 from an experiment designed to test 'streamer' formation. The results are suggestive until $O$ (40 days), after which the numerical streamers become too broad to match with observations. 
satellite observations. To clarify the degree to which our model can predict the structure of streamers, we show an advection-diffusion experiment in which the dynamic Ring was released in the vicinity of an east-west oriented tracer field. The results of this experiment are presented in Fig. III.16, which shows the growth of a streamer. For short times, the pictures strongly resemble those from satellites, Indicating that Rings are capable of the streamer production by advection. After about 30 days, however, the numerical streamers thicken and the pictures lose their resemblence to the oceanic streamers, which are observed to remain thin.

\section{III.f Summary-}

In this chapter, we have investigated the Lagrangian flows associated with dynamical models of Gulf Stream Rings. The governing equation was the equivalent barotropic equation, which expresses the quasi-conservation of potential vorticity along fluid paths; the only non-conservative force in operation was viscosity, which was compelled to be weak. It was found that the evolution of the Ring model was slow enough to allow accurate predictions of particle trajectories to be made by using Flierl's (1981) formula for streaklines. The shapes of the particle trajectories were somewhat different than those of the radially symmetric pattern used in earlier kinematic studies. The present study predicts that the fluid west of a Ring is most likely to mix into the trapped zone, and that the Lagrangian fields are east-west asymmetric. The cause of the asymmetry was shown to be the high and low pressure centers created by the evolving Ring, and it was argued from a dynamical 
point of view that their presence represented the corrections necessary to improve earlier kinematic models.

Particle trapping and the exterior particle trajectories were investigated from the standpoint of potential vorticity, and it was found that dissipation was important to the shape of the $q$ contours near the trapped zone. The results suggested trapped zone-exterior exchange is weak.

Finally, we discussed experiments which demonstrate the ability of Rings to account for certain satellite observed sea surface temperature patterns. The tendency for warm core Rings to develop 'tails' was successfully replicated, as was the production of 'streamers', or cold water advection from the Shelf to the Slope.

Although we were able to duplicate streamer production, there are still some unanswered questions about their long term evolution. Basically, streamers are observed to remain thin; that is, after the cold water is advected south in a narrow tongue, they do not spread laterally, as in Fig. III.16. Rather there appears to be some mechanism at work which keeps the anomalous temperatures associated with the streamers confined to a narrow tongue. One possible explanation for this phenomenon takes into account air-sea interaction; if cold surface water is exposed to a warm atmospheric state, the surface temperature anomaly is removed (see Chapter IV). A hypothetical scenario for the Shelf waters would have them advecting into the Slope via streamer production, where the warm air would erase their cold sea surface 
signature. The Shelf waters, with all of their unique properties, would then be injected into the Slope, but thermally modified in the process, so that they become invisible to satellite infra-red sensors. This is, of course, speculation; whether we are discussing a viable physical mechanism will be the subject of later study. 
CHAPTER IV. AN ANNUAL MIXED LAYER MODEL WITH APPLICATION TO GULF STREAM RINGS

IV.a. Introduction-

Just after formation, the sea surface temperatures (SST's) of Gulf Stream Rings are different by several degrees from those of the surrounding water, with cyclonic Rings distinguished by cold SST and anticyclonic Rings by warm. From satellite sea surface imagery, we have discovered that the subsequent evolutions of warm and cold Ring SST's are different. Quite simply, cold core Rings lose their cold signatures while warm core Rings do not (the Ring Group, 1981). Other features of warm Ring SST behavior include a tendency for their temperature anomaly to weaken in the summer, but reemerge in the fall (Friedlander, personal communication). Whether cold Rings do the same is less clear. In any case, the 'survivability' of warm anomalies is apparently much greater than that of cold, and in this chapter, we will attempt to understand why this is so.

Background-

Rings are capable of particle trapping and the residence times for trapped fluid are is estimated to be long (Chapter III and Flierl and Dewar, 1981). Therefore, for most of its lifetime, the upper layer core 
waters of a Gulf Stream Ring are primarily those of its parent region. Repeated hydrographic surveying of individual Rings has verified this. Still, Rings have been observed to intermittently interact with the Gulf Stream (Richardson, 1980), resulting the injection of different water types into the core. There currently is some question as to whether such interactions are normal, but in any case they leave the general cross-Ring 1sothermal displacements unaltered.

Consider warm core Ring formation and propagation from the reference frame of a fluid column, inftially in the Sargasso, which ends up in the trapped zone. Prior to formation, the fluid is subjected to Sargasso Sea air-temperatures. As the Ring is formed, the atmosphere becomes less Sargasso-like until at separation, and from then on, it is Slope Water in nature. A comparison of local monthly mean temperatures (Marine Climatic Atlas) peculiar to the Sargasso Sea and Slope Water shows them to be very different (See Fig. IV.1.a). The air above the Sargasso is warmer $\left(\overline{\mathrm{T}}_{\text {sarg }}=20^{\circ} \mathrm{C}\right)$ than that over the slope $\left(\overline{\mathrm{T}}_{\text {slope }}=\right.$ $10^{\circ} \mathrm{C}$ ) and is comparatively moderate in annual variation ( 8 Tsarg $=10^{\circ} \mathrm{C}$, $\left.{ }^{r} T_{s l o p e}=20{ }^{\circ} \mathrm{C}\right)$. This contrast is characteristic of these two regions (see Fig. IV.1.b and $c$ ) and a plausible explanation for it is that the air is warmed by the Gulf Stream. To the column of water, Ring formation is manifested as a transition from warm to cold airtemperature, and the evolution of its sea surface temperature should reflect the change. Similarly, a cold to warm transition will be experienced by the surface layer of a cold core Ring. We will compute the response of 'typical' Slope Water and Sargasso mixed layers to Sargasso and Slope Water forcing, respectively, in an effort to explain the SST evolution of warm and cold Rings. 


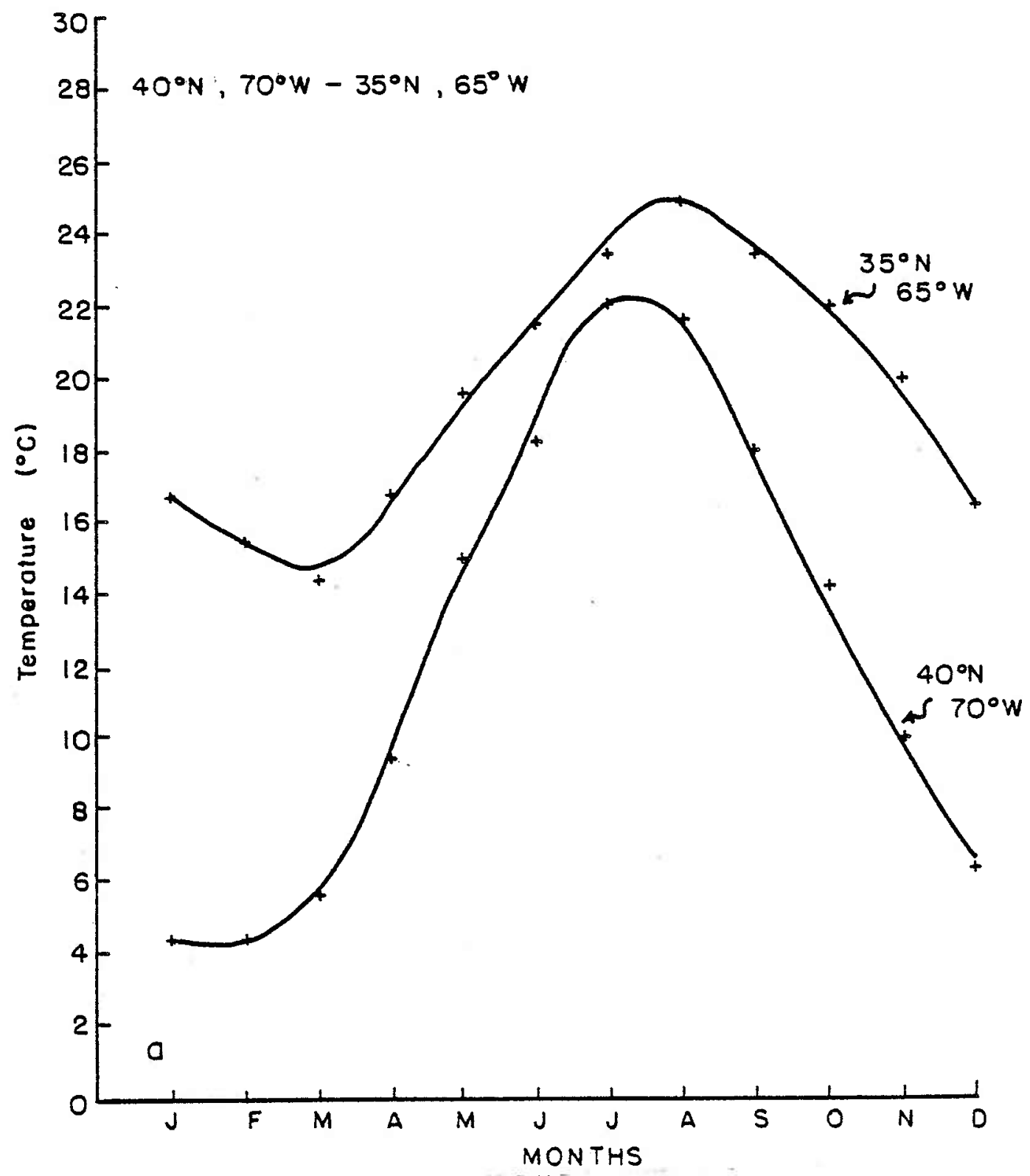

Figure IV.1. Comparison of Air-Temperature Cycles

In (a), we compare monthly mean air-temperatures from the Slope and the Sargasso. Note that for the entire year, the Sargasso air is warmer than the Slope, while the Slope is characterized by much greater variation. Both the Slope are Sargasso station locations were chosen because they correspond to points of frequent Ring observation. This difference in air temperature is characteristic for most of the slope Water and Sargasso, as demonstrated in (b) and (c), where we display average air temperatures in January and July. In the winter, the contrasts are great, and in the summer, weak. 


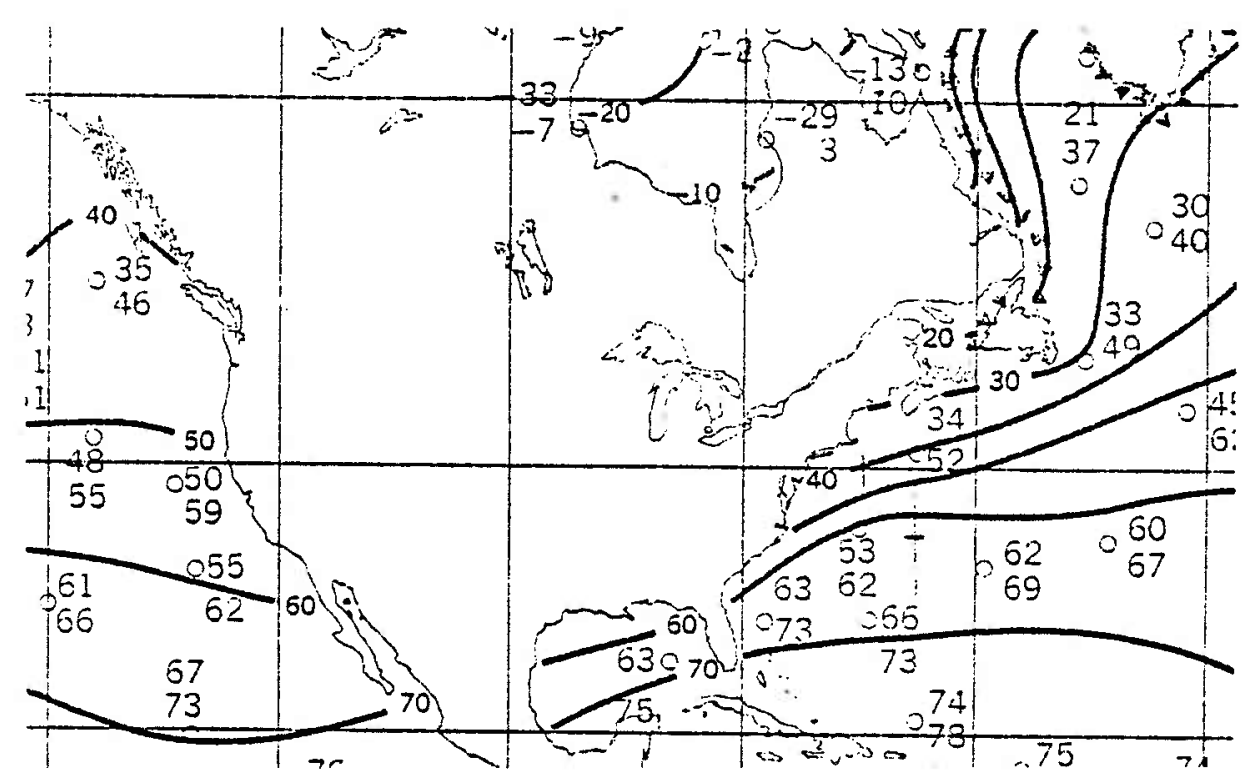

b

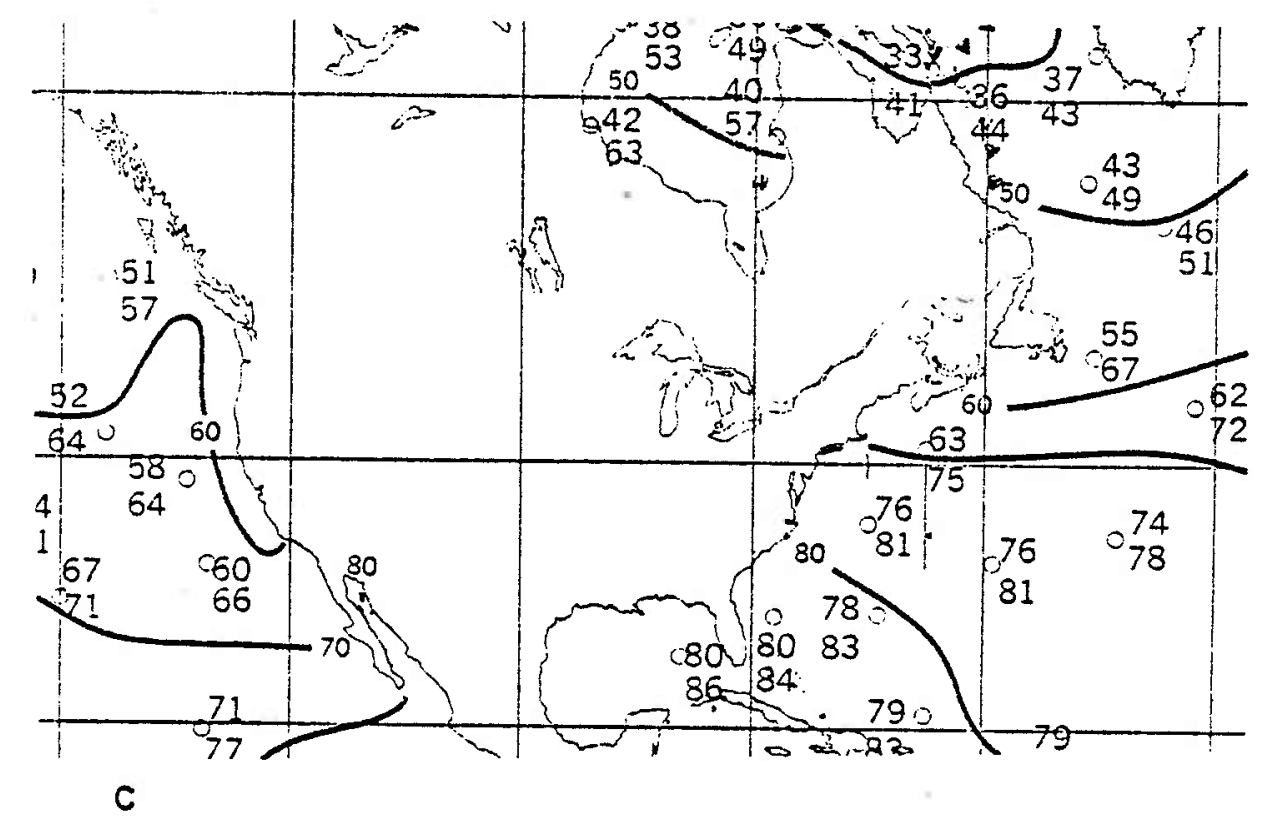


Note that, in this model, the heat budget of a fluid column is determined by the flux through its surface, so we will employ a one-dimensional mixed layer model. A good question to ask is whether we can expect $1-d$ models to be adequate, especially in view of their poor performance in other Ring problems (Schmitt, personal communication). One possible error comes from Ring decay, in which there is a mass transport into the trapped zone and with it a heat flux. We consider the size of this flux in comparison to the surface fluxes. Approximating the Ring by a cylinder, in which case the inflow, $u_{r}$, is related to the downwelling, w, by:

$$
u_{r}=w_{o} / 2 h_{o},
$$

where ro is the radius of the trapped zone, and ho the mixed layer depth, the ratio of the radially directed heat flux to the surface flux is:

$$
u_{r b_{r}} /\left(B f l u x / h_{0}\right)=w \gamma b b /(2 \text { Bflux }) \text {. }
$$

Using an average $w$ of $10^{-4} \mathrm{~cm} / \mathrm{sec}, \zeta b$ of $1 \mathrm{~cm} / \mathrm{sec}^{2}$, and $a$ Bflux of $10^{-3} \mathrm{~cm} 2 / \sec ^{3}$, this ratio is:

$$
\mathrm{urbr} /\left(\mathrm{Bflux} / \mathrm{ho}_{\mathrm{O}}\right)=.05 \ll 1 \text {, }
$$

and may therefore be neglected. It is with less justification that we ignore the heat flux due to the intermittent interactions with the Stream. In those applications where $1-d$ models have failed, the occurrence of such events have upset the budgets. In support of the aptness of $1-d$ models for the problem at hand, we appeal to the fact that we are trying to explain an annual, and therefore most likely deterministic, cycle in SST. Also, the results are most dependent on the cross-Ring isopycnal structure, which is relatively unaffected by interactions with the Gulf Stream or the Shelf Water. The solutions 
will suggest that one dimensional,models include the processes necessary to explain the relative 'robustness' of warm Ring SST, as well as the summertime loss and fall reemergence of Ring thermal structure.

IV.b An Annual Mixed Layer Mode1-

Mixed layers are regions of very complicated, turbulent activity, and exhibit variability on the time scales of seconds to years. Many successful mixed layer models, known as 'bulk models', consist of a surface layer overlying an 'Intermediate layer' (see Fig. IV.2), and exploit the 'well mixedness' of the surface layer to compute the turbulent Reynolds stresses in terms of the boundary conditions. An example of the mixed layer development computed by a bulk model (Pollard, Rhines, and Thompson, 1973) is included in Fig. IV.3 (see Appendix A.IV for details). These figures each contain 10 buoyancy profiles at 10 day intervals, and range from fall mixed layers (a) to summer mixed layers (c). Perhaps the most noticeable occurrence in these graphs is the sudden reorganization of the thermocline at a shallow depth in early spring, followed by the development of a thin, warm layer. Notice that the depth of this layer remains almost constant ( $b$ and $c$ ). Proceeding downward from the mixed layer base in $b$, the rate at which the fluid is being heated rapidly decreases. The mixed layer maintains a density discontinuity with respect to the intermediate layer, and the intermediate layer itself develops strong gradients with depth. In Fig. IV.3.a are typical model generated buoyancy-depth profiles from fall/winter. The interesting behavior here concerns the mixed layer depth and the buoyancy discontinuity at its 


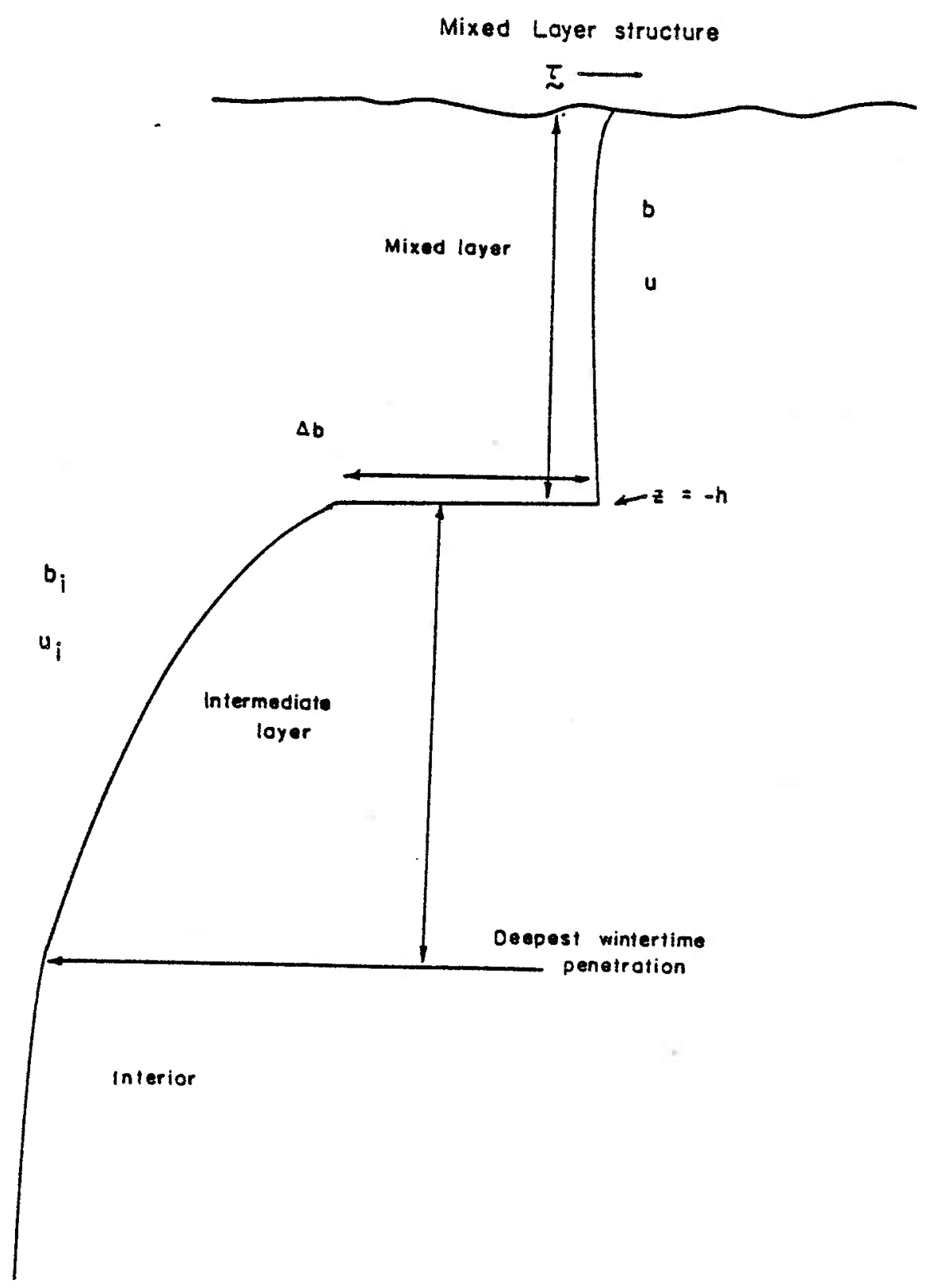

Figure IV.2. Structure of Bulk Mixed Layer Models

The upper waters are divided into a well-mixed layer, in direct contact with the atmosphere, and an intermediate layer. The vertical extent of the intermediate layer is defined by the deepest penetration of the wintertime mixed layer. 


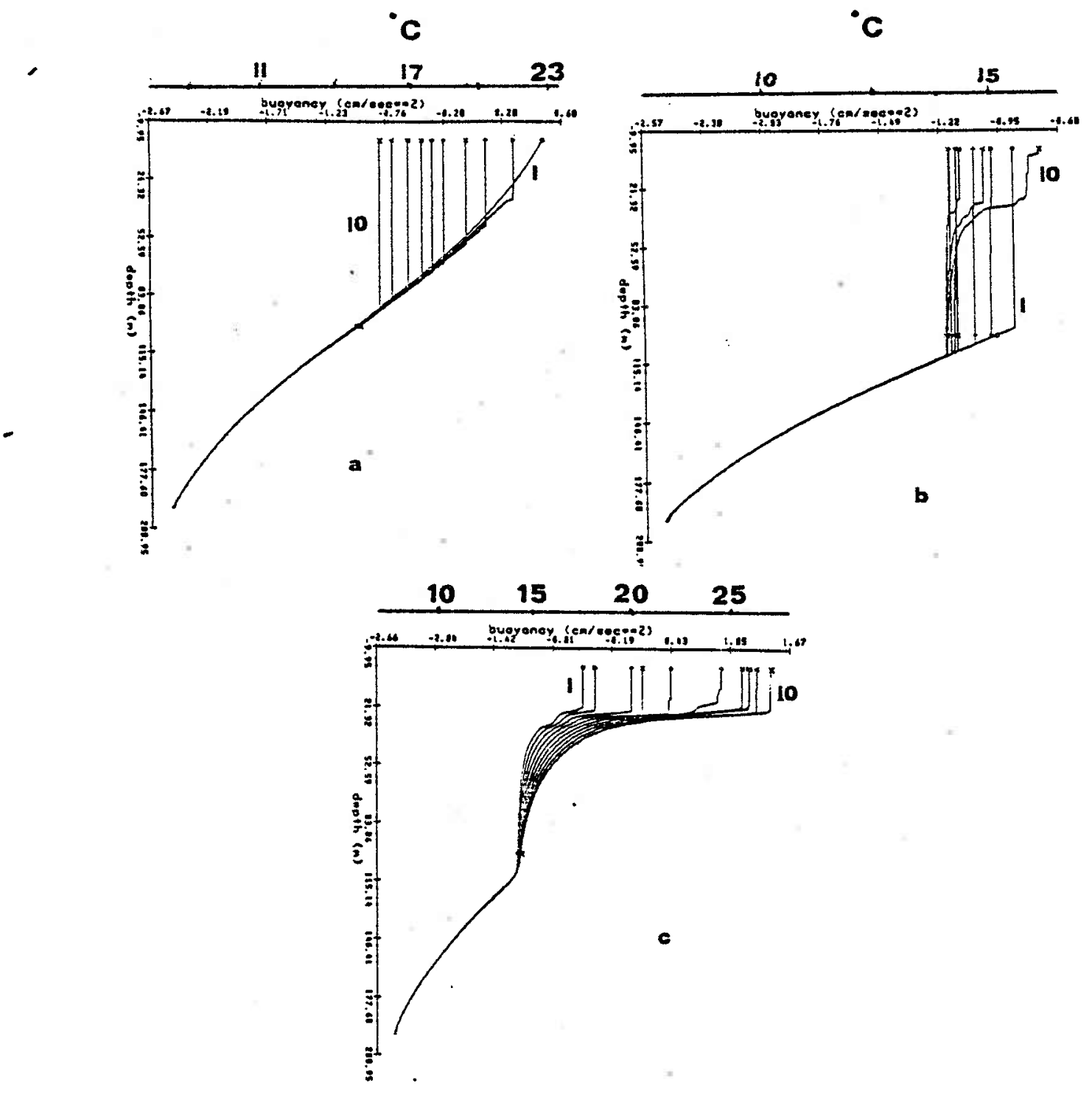

Figure IV.3. Temperature Traces from the PRT Bulk Model

Each plot contains 10 traces at intervals of 10 days, and all three together cover 300 days worth of mixed layer evolution. Of interest is the summertime mixed layer, which develops nearly as a constant depth layer, and the fall degradation of the seasonal thermocline. Note also the change in imtermediate layer structure from (a) to (c), showing that over one year, this layer has lost heat. Compared to (a), wintertime cooling must erode a much stronger seasonal thermocline in (c) prior to very deep layer formation, and therefore the upcoming winter will extract less heat from the intermediate layer than the previous winter. 
base, which quickly fades with increasing h. From our point of view, this is equivalent to determining mixed layer depth by matching the intermediate layer buoyancy to that of the mixed zone, suggesting a dependence of wintertime SST on deep buoyancy structure.

In this model, the mixed layer depth, $h$, is governed by the direction of the heat flux. In the summer, the surface waters become warm and buoyant, and the requirement of mixing light water downward effectively isolates the surface layer from the deep. The well-mixed zone subsequently evolves almost as a constant depth layer, underneath of which develops a very strong buoyancy gradient. In the wintertime, buoyant convection, driven by the production of cold, heavy surface water, aids in the mixing process. The seasonal thermocline is eroded, and the mixed layer penetrates into the deep buoyancy structure. The great depths of the wintertime mixed layer preclude either a sizeable heat flux or buoyancy jump at the mixed layer base. As air-sea heat exchange switches sign in the spring, buoyant convection halts and a thin mixed layer develops anew. We also note that the intermediate layer is heated year round by penetrative radiation (see Appendix A.IV).

A set of equations describing the seasonal character of the mixed layer/intermediate layer is:

$$
h b_{t}=\tilde{f}(b-b a)+B_{a s}-B_{a}(-h), \quad \text { Eq. IV.1.a }
$$

where $h$ is the mixed layer depth, $b$ the buoyancy, $b_{a}$ the atmospheric 
buoyancy, defined by:

$$
b_{a}(t)=g \Re\left(T_{a}-T_{0}\right)
$$

$B_{a s}$ the average radiative surface heat $f$ lux, and $B_{a}(-h)$ the average irradiant heat flux which enters the deep ocean through the mixed layer base (see Table IV.I). The intermediate layer buoyancy, $b_{1}$, is governed by:

$$
\mathrm{b}_{\text {it }}=\mathrm{Baz}_{\mathrm{az}} \quad \text { Eq. IV.1.b }
$$

where $B_{a z}$ is penetrative radiation. The mixed layer equation is closed by a specification for $h$, namely:

$$
h=h_{0} \quad \text { Eq. IV.1.C }
$$

(a constant) during 'spring' and 'summer', or:

$$
b=b_{1}(-h) \quad E q . I V \cdot 1 . d
$$

during 'fall' and 'winter'. 'Seasons' are delimited by the sign of:

$$
F=-\tilde{f}\left(b-b_{a}\right)+B_{a s}-B_{a}(-h) \text {, }
$$

i.e. spring/summer if $F$ is positive, and fall/winter if negative. In the present calculations, we have ignored the seasonal dependence of penetrative radiation, approximating these terms by a constant (see Table IV.1).

Similar equations were used by Warren (1973), although in his model, there was no 'mixed' layer. Incoming heat was distributed Iinearly with depth. We have chosen the present heat distribution from an examination of the bulk model, so we believe it is more dynamically consistent. Analytical justification for the time evolution of $h$ is contained in Appendix B.IV. The accuracy of Eqs. IV.I is the subject of Appendix C.IV, with the results that they reproduce the bulk model averaged SST and mixed layer depth surprisingly well. 
Table IV. 1

Symbol Definitions and Scales

\begin{tabular}{|c|c|c|c|c|c|}
\hline Symbol & Definition & Value & Symbol & Definition & Value \\
\hline \multicolumn{2}{|c|}{ Environmental } & \multirow[b]{2}{*}{$\begin{array}{l}10^{-4} \sec ^{-1} \\
2 \times 10^{-4} \\
{ }^{\circ} \mathrm{C}^{-1}\end{array}$} & \multirow{3}{*}{$\begin{array}{c}g . . . \\
P o \\
\ldots \ldots\end{array}$} & \multirow{3}{*}{$\begin{array}{l}\text { gravity..... } \\
\text { reference } \\
\text { water } \\
\text { density..... } \\
\text { time....... }\end{array}$} & \multirow{3}{*}{$\begin{array}{c}10^{3} \mathrm{~cm} / \mathrm{sec}^{2} \\
1 \mathrm{gm} / \mathrm{cm}^{3}\end{array}$} \\
\hline $\begin{array}{c}f_{0} \ldots \\
r \\
z \\
\ldots\end{array}$ & $\begin{array}{l}\text { Coriolis } \\
\text { coefficient.. } \\
\text { coefficient } \\
\text { of thermal } \\
\text { expansion... } \\
\text { vertical } \\
\text { coordinate... }\end{array}$ & & & & \\
\hline \multicolumn{2}{|c|}{ Atmospheric } & & & & \\
\hline 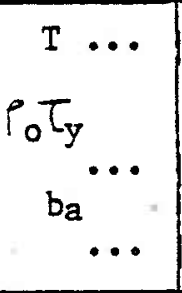 & $\begin{array}{l}\text { temperature.. } \\
\mathrm{N}-\mathrm{S} \text { wind } \\
\text { stress....... } \\
\text { atmopheric } \\
\text { buoyancy..... }\end{array}$ & $\begin{array}{l}\text { variable } \\
\text { variable } \\
\text { variable }\end{array}$ & $\begin{array}{l}\mathrm{u}_{\mathrm{a}} . \\
\mathrm{P}_{\mathrm{a}} \\
\ldots\end{array}$ & $\begin{array}{l}\text { E-w wind.... } \\
\text { stress } \\
\text { wind........ } \\
\text { velocity } \\
\text { air } \\
\text { density }\end{array}$ & $\begin{array}{l}\text { variable } \\
\text { varlable } \\
10-3 \mathrm{gm} / \mathrm{cm}^{3}\end{array}$ \\
\hline \multicolumn{6}{|c|}{ Mixed Layer } \\
\hline $\begin{array}{l}\mathrm{h} \\
\ldots \\
\frac{\mathrm{u}}{\mathrm{h}_{0}} \\
\cdots\end{array}$ & $\begin{array}{l}\text { mixed layer } \\
\text { depth......... } \\
\text { velocity } \\
\text { summer time } \\
\text { depth......... }\end{array}$ & $\begin{array}{l}\text { variable } \\
\text { variable } \\
30 \mathrm{~m}\end{array}$ & $\begin{array}{l}\text { b... } \\
\text { T... } \\
\mathrm{h}_{1}\end{array}$ & $\begin{array}{l}\text { buoyancy.... } \\
\text { temperature. } \\
\text { depth for } \\
\text { wintertime } \\
\text { sample } \\
\text { problem.... }\end{array}$ & $\begin{array}{l}\text { variable } \\
\text { variable }\end{array}$ \\
\hline \multicolumn{6}{|c|}{ Interior } \\
\hline$\Gamma \ldots$ & $\begin{array}{l}\text { buoyancy..... } \\
\text { buoyancy } \\
\text { gradient ..... }\end{array}$ & $\frac{10^{-4}}{\sec ^{2}}$ & $\begin{array}{c}u_{1} . . \\
\mathrm{N}^{2} \\
\ldots\end{array}$ & $\begin{array}{l}\text { velocity.... } \\
\text { buoyancy } \\
\text { frequency... }\end{array}$ & variable \\
\hline
\end{tabular}


Table IV.I Symbols and Definitions (continued)

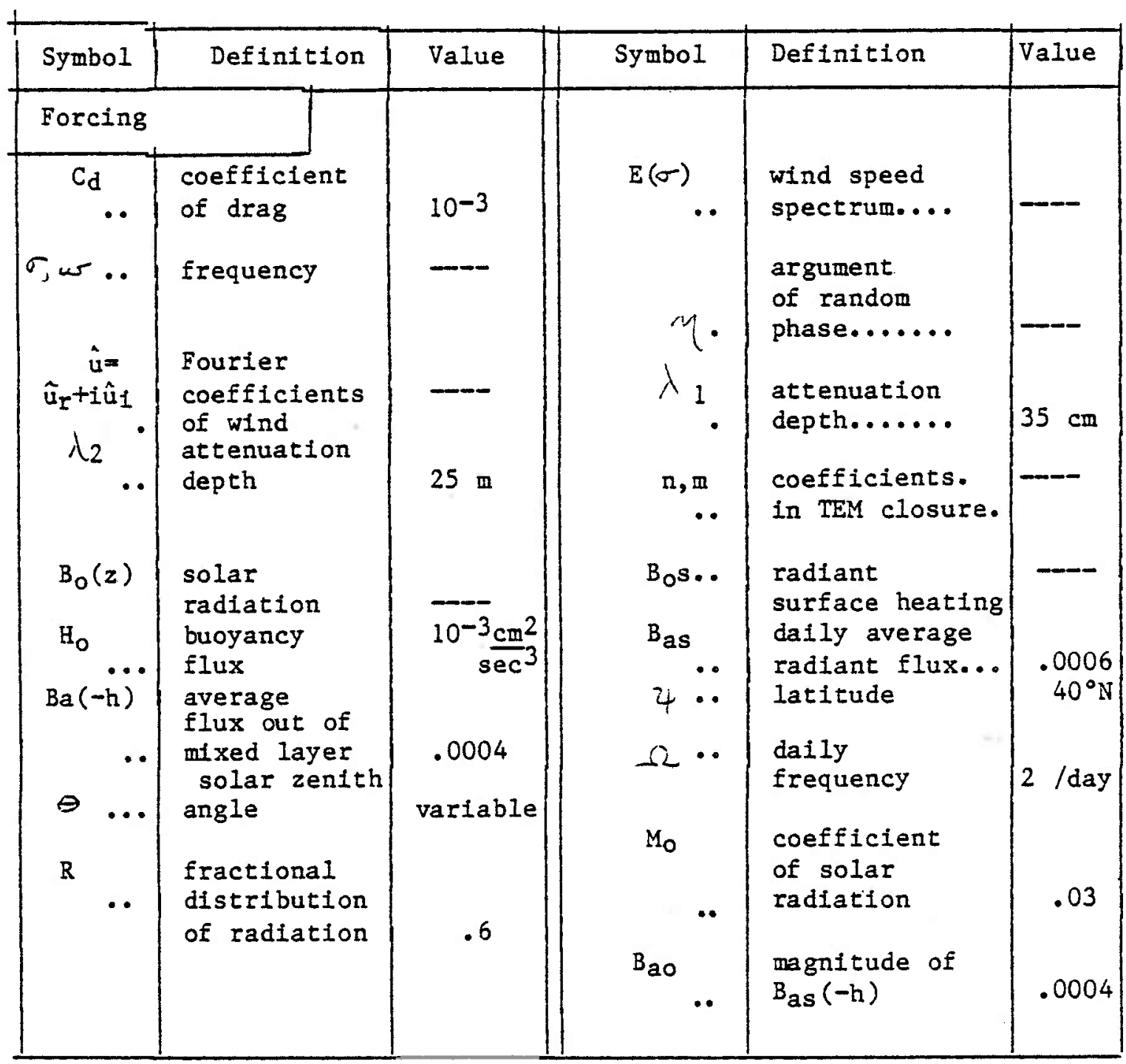


In the experiments to be discussed, the atmospheric annual temperature cycle, $b_{a}(t)$, will take the general form:

$$
b a(t)=a_{2}+a_{1} \cos (\omega t+\pi) \quad E q \cdot I V \cdot 2
$$

with the maximum temperatures occurring at an annual phase of $\pi . B_{a}$ is taken as:

$$
B_{a}=B_{a 0} \exp \left(z / \lambda_{2}\right)
$$

(see Appendix A.IV). Note, there is no explicit reference to wind stress. The effects of the wind, however, enter through both the value and maintenance of the sumertime mixed layer depth, $h_{0}$, and the production of the seasonal thermocline with the onset of spring. $h_{0}$ was chosen to be $30 \mathrm{~m}$; the rationale behind this choice is contained in Appendix C.IV.

IV.c Limit Cycle Calculations-

The first step to understanding Ring SST is to determine the average mixed layer characteristics of the parent region. An exact definition of 'average' does not exist; however, we have found that, subject to a choice of ba, Eqs. IV.l possess limit cycle solutions. If we assume that the average residence time of fluld in each region is long, we can interpret the limit cycles as 'average' mixed layers. This definition turns out to be impractical, for the limit cycles of Eq. IV.I represent mixed layers which deepen to infinity at the end of winter. This is an undesirable feature, but it turns out to be inimportant. We can st1ll obtain accurate estimates for 'average' mixed layers in terms of 'quasi'-limit cycle solutions as follows. 
The infinite deepening of the late winter part of the limit cycle is a consequence of the form of the penetrative radiation, because the heat balance at any level is:

$$
b e=-\overline{\left(w^{\prime} b^{\prime}\right) z}+B a z \quad \text { Eq. IV. } 3
$$

(see Eq. II.26), i.e. between turbulent heat fluxes and penetrative radiation. For any depth to be cycling in a limit state;

$$
T^{-1} \int_{2}^{2 \pi / \omega_{0}} b_{t} d t=0
$$

At every level, there is an annual balance between turbulent heat flux and radiation. The form we have chosen for the penetrative radiation is non-zero at all depths; therefore, at all depths turbulent heat flux (deep winter time mixing) is required.

In the real ocean, radiation is probably not significant beyond the first few hundred meters, after which the present parameterization of radiation is suspect (Simpson and Dickey, 1981). In an effort to correct this fault, a series of experiments were performed in which radiation was expunged at some ad-hoc depth, hc. Notice that according to the previous arguments, whatever depth is chosen as the radiation cut-off also becomes the depth of deepest winter time mixing. Several cut-off depths were tested, giving the result that sea surface temperature was unaffected so long as hc was much greater than the e-folding scale of the radiation. In the present set of calculations, we returned to the original formulation, $h c=\infty$. The 'typical' mixed layers, referred to earlier as 'quasi'-limit cycle mixed layers, were obtained by integrating Eqs. IV.1 until the numerical cycle was repeating itself to within a few parts in several decimal places (see FIg IV.4). 
Two properties of limit cycles which are important to the problem at hand are existence and uniqueness. Both are difficult to prove, but apparently apply to the limit cycles of the present problem. For example, in all numerical experiments with the same atmospheric forcing, $b_{a}(t)$, the solutions, regardless of initial condition, converged towards the same (and therefore apparently unique) limit cycle. With regards to existence, we note that the effect of the forcing is to adjust the deep buoyancy profile towards limit cycle behavior. This may be seen from Eq. IV.3. Consider a non-limit cycle intermediate layer, in which therefore at some depths there are net annual imbalances of heat. The effect of solar radiation is always to warm the water, and that of wintertime mixing to cool it. Thus, if at some depth there is a net loss of buoyancy, that depth was 'in' the wintertime mixed layer for too long a time. Note, however, that the resulting colder profile prevents the upcoming wintertime mixed layer from deepening as efficiently as the previous winter (see Fig. IV.3). Hence in the winter to follow the intermediate layer depths will be cooled less. A net heat gain in the intermediate layer produces a buoyancy profile which allows deep mixing to occur earlier during winter, and therefore extract greater amounts of heat from the intermediate layer in the next year. Either shift is closer to an annual heat balance, which is the characteristic of a limit cycle.

'Typical' Mixed Layers-

In Figs. IV.4, we graph mixed layer buoyancy, b, against mixed layer depth, $h$, as a measure of the limit cycle behavior from both the Sargasso and the. Slope. The air-temperature cycles for each region were 
taken to be:

$$
\operatorname{Ta}(t)=10^{\circ} \mathrm{C}+10^{\circ} \mathrm{C} \cos (\omega t+\pi)
$$

for the Slope, and:

$$
\mathrm{Ta}(t)=20{ }^{\circ} \mathrm{C}+5{ }^{\circ} \mathrm{C} \cos (i \omega t+\pi)
$$

for the Sargasso (see Figs. IV.1). Notice that Figs. IV.4 actually contain four years worth of model data; the cycle is repeating to at least the width of the plotting line (however, observe the point of deepest penetration). Finally in Fig. IV.5, we have included several model generated lower layer (i.e. depths greater than $30 \mathrm{~m}$ ) temperature profiles. The Slope Water is characterized by a much greater range of SST than the Sargasso, while the Sargasso profiles are much warmer. Compare the model profiles with actual winter and summer XBT traces (Fig. IV.6) taken at locations within the Slope Water and Sargasso where Rings are frequently observed.

Finally, we note that the SST extremes as predicted by the limit cycles match well with observations. The coldest Slope Water SST is typically $5{ }^{\circ} \mathrm{C}$, as compared to $6^{\circ} \mathrm{C}$ in the model, and the warmest is 19 ${ }^{\circ} \mathrm{C}$, compared to the model value of $20^{\circ} \mathrm{C}$ (Colton and Stoddard, 1972). In the Sargasso south of the Gulf Stream, the range is observed to be from $20{ }^{\circ} \mathrm{C}$ to $26{ }^{\circ} \mathrm{C}$ (Fuglister, 1947), and the model predicts $20{ }^{\circ} \mathrm{C}$ to $27{ }^{\circ} \mathrm{C}$.

IV.d Adjustment Calculations-

Given the parent region mixed layer structures, we can compute their evolution when subjected to host region forcing. Recall that the sudden change of atmospheric state is meant to model Ring formation. 


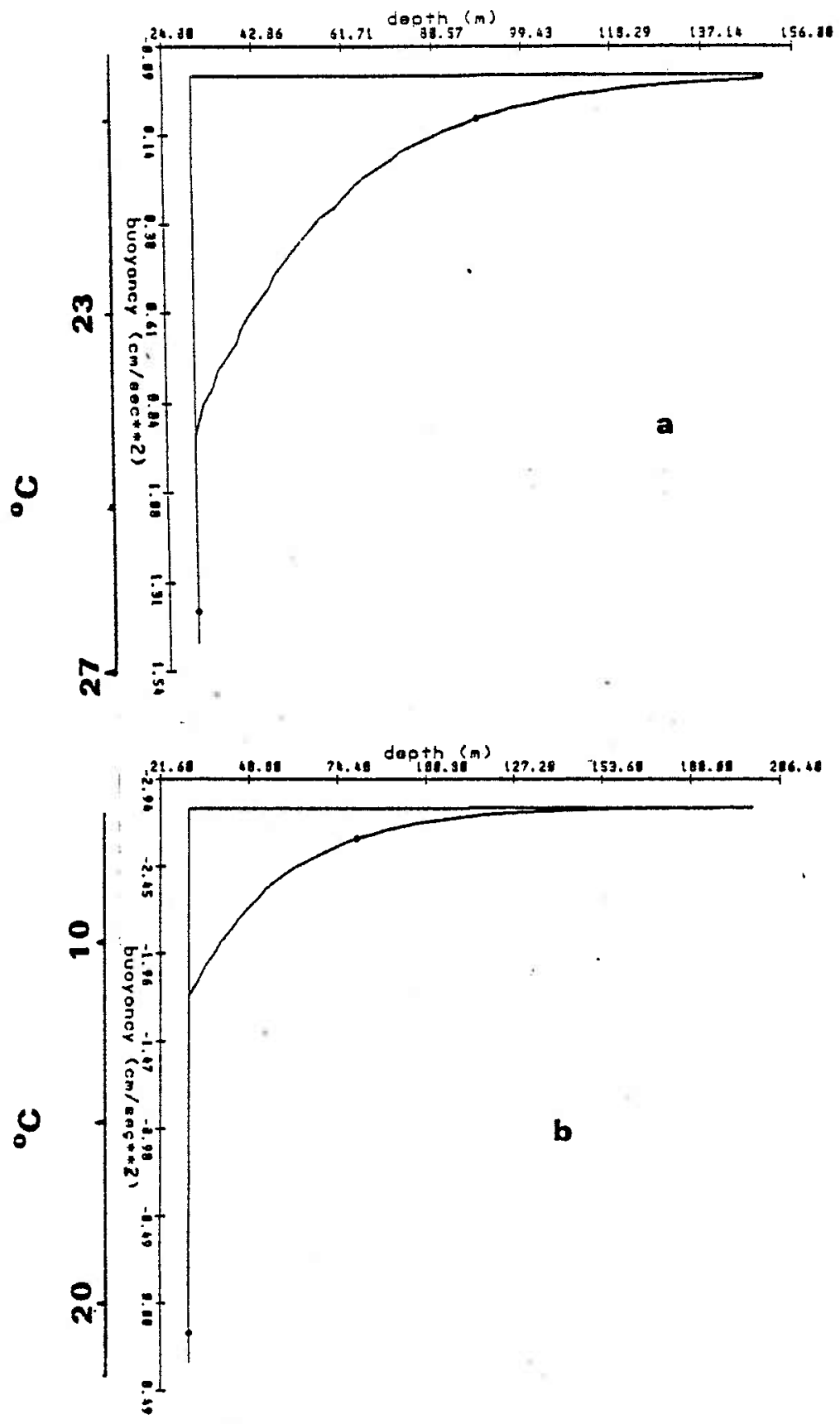

Figure IV.4. b Versus h Limit Cycles

Here we plot the limit cycle solutions for the (a) Sargasso and the (b) Slope Water. Each graph contains four consecutive years of data, from which it is apparent that, for the most part, the cycle is repeating to a few parts in several decimal places. In (a), the cycle is still weakly evolving at the depth of deepest wintertime penetration. Note the greater range of the Slope water mixed layer temperature in comparison to the warmer Sargasso. 
Page $-106 \%$

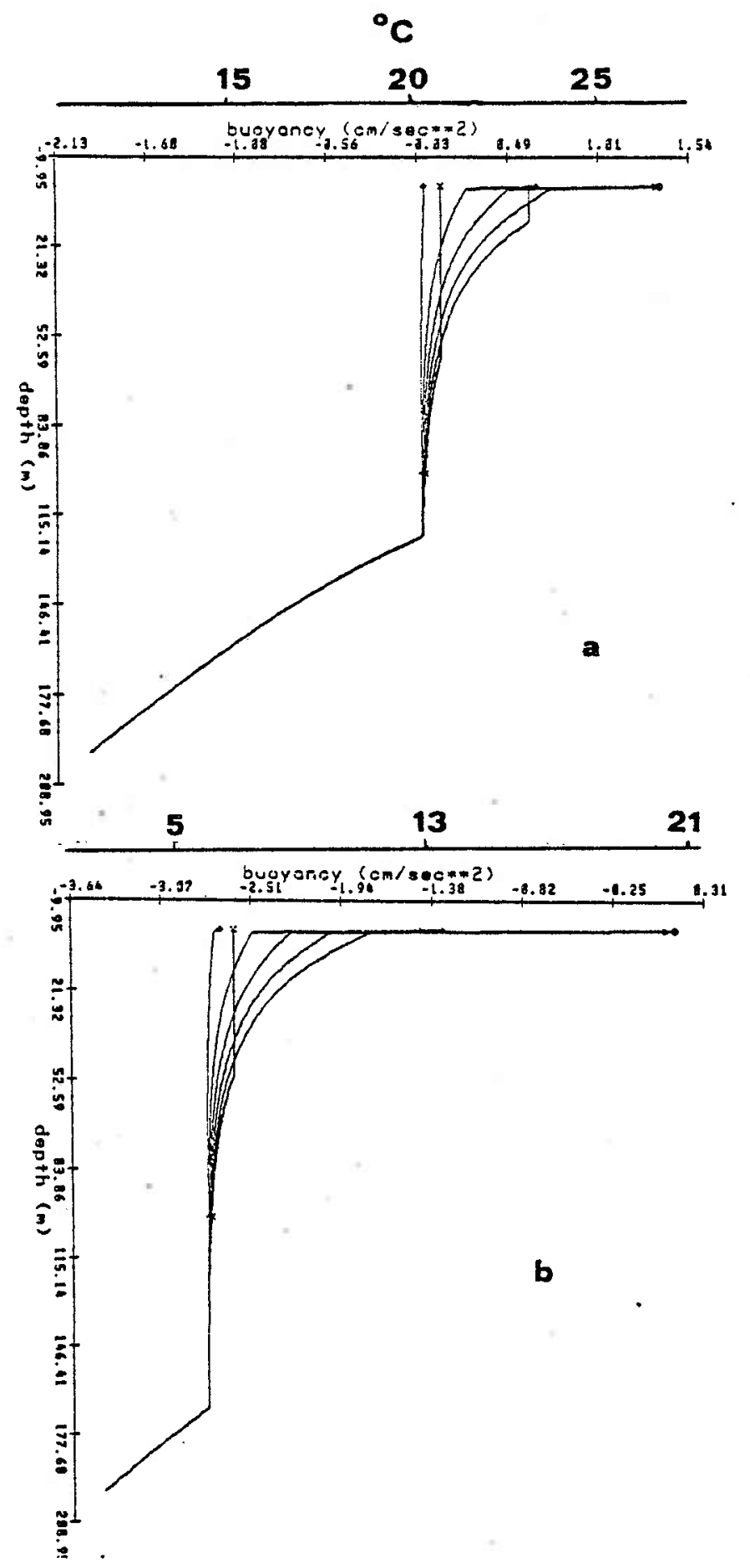

Figure IV.5. Lower Layer Buoyancy Structure

Here we have plotted, in (a) and (b), the buoyancy traces for depths greater that $30 \mathrm{~m}$ from limit cycles (a) and (b), respectively, of Fig. IV.4. In the present model, the mixed layer is never shallower than $30 \mathrm{~m}$, so this portion of the column was left out. The time interval between traces is two months. 

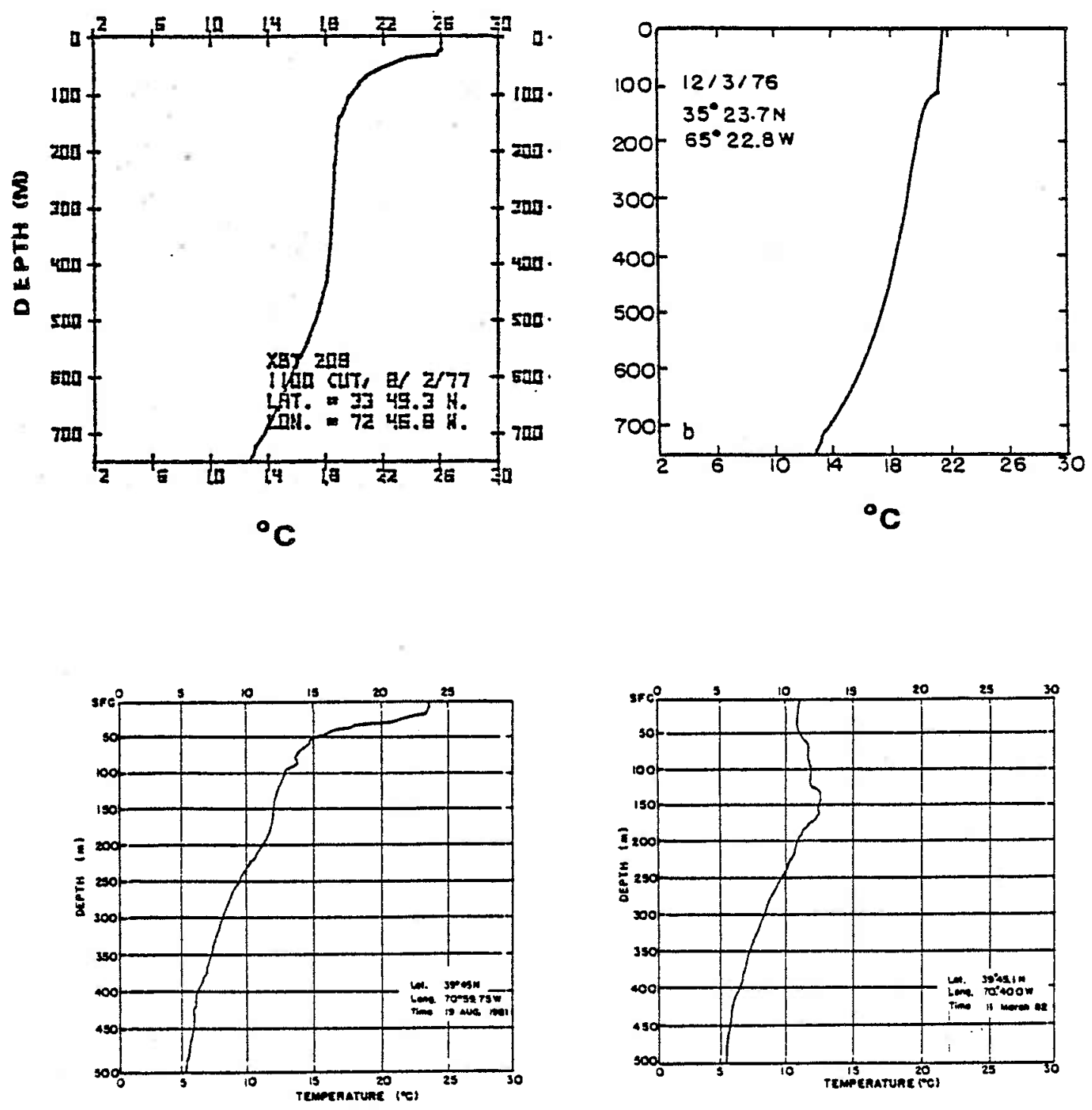

Figure IV.6. Summer and Winter Slope and Sargasso XBT Measurements

Here are typical upper layer temperature traces from both the Sargasso and the Slope. In agreement with the limit cycle buoyancy traces, the range of the Slope SST is much greater than that of the Sargasso, but the Sargasso is much warmer. 
In Fig. IV.7, we compare the annual cycles of SST for a warm Ring central mixed layer and a 'typical' Slope Water mixed layer. Included are six years worth of Slope Water SST, which is compared to two years worth of typical Sargasso SST and four years worth of warm core response. Note however that Rings are generally only in the host region for 1-2 years, so the first few years of response are the most applicable. In this experiment the Ring was formed at the warmest point in the atmospheric cycle. Notice the temperature contrast is greatest in winter, and is roughly $6{ }^{\circ} \mathrm{C}$. As the year progresses, this contrast first weakens, disappears, and then reemerges. The same information is plotted in Fig IV. 8 for the analogous case of a cold Ring. Note that the cold Ring wintertime temperature contrast $\left(O\left(1^{\circ} \mathrm{C}\right)\right)$ is much weaker than that of the warm core Ring. $1^{\circ} \mathrm{C}$ is roughly the error of satellite measured SST, so these results suggest that in infra-red images, warm Rings would be much more evident than cold. As before, summertime erases the cold Ring temperature contrasts, and the onset of the fall rejuvenates them. Recall that the diabatic forcing parameters were chosen to agree with data; therefore, we should attach significance to the values which the model generates.

The reasons for the difference in the SST contrasts are essentially contained in the buoyancy profiles from each experiment (Figs. IV.9 and 10). Exposing a 'typical' Sargasso Sea mixed layer to the cold Slope Water wintertime results in unusually deep surface layers, produced mostly by convective mixing. Hence, warm core mixed layers respond slowly in temperature; equal extractions of heat produce lesser decrements in SST owing to the thickening layer from which the heat is 


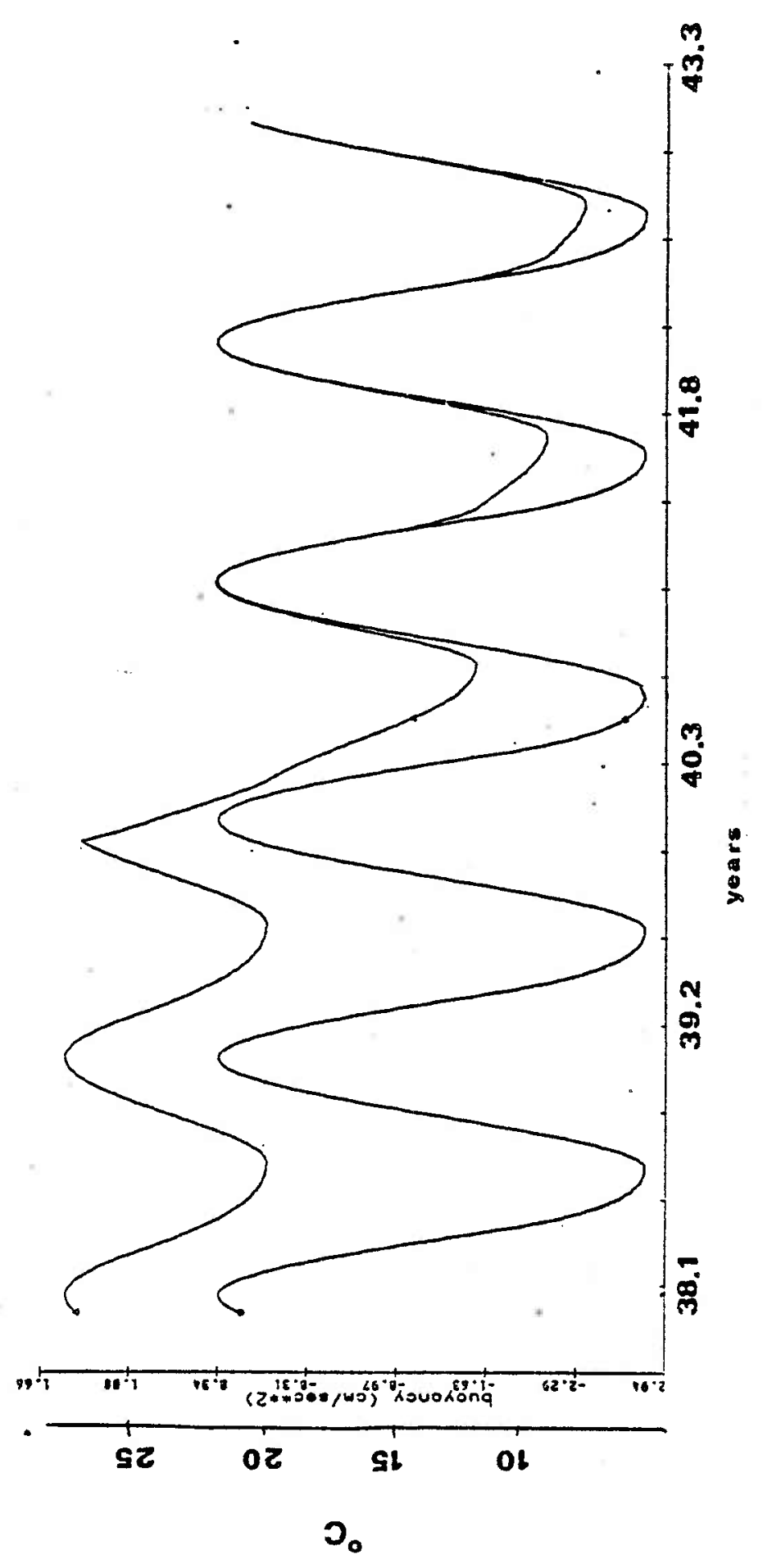

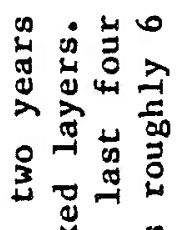

車 常

車幽

崖出出

- 48 宛各 os os 0 능 记

\& $0 \underbrace{\infty}$

C 0 ह

(⿻)

$\infty$

○菲出

ङ

떵

-

की

可每

峞刍

ठำ

$\infty$ फ记

훙 ⿷匚⿱乛耳

ㄷ. 040

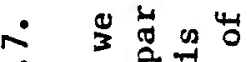

¿

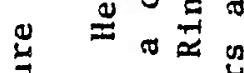

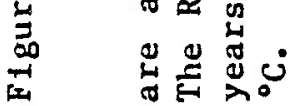




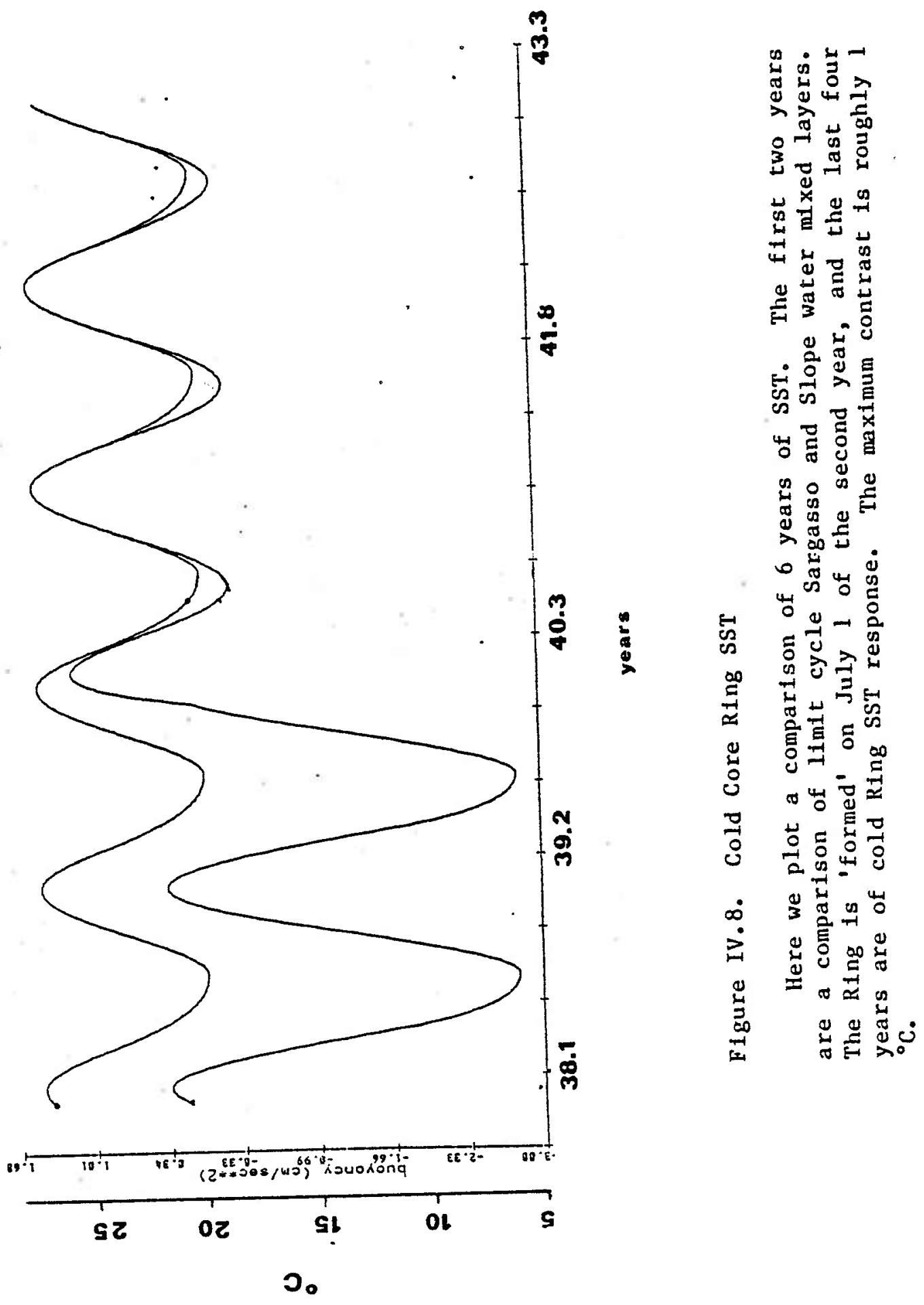


removed. Estimates of warm. core mixed layer relaxation times are generally longer than the duration of winter; therefore, being initially warm, the Sargasso mixed layers simply maintain their warm identity throughout the cooling season. As winter gives way to summer, thin layers of depth $h_{0}$ develop and shortly thereafter the mixed layers lose memory of their early spring-time buoyancy. Note that although surface contrasts are covered up in roughly a month, the deep thermal structure retains its warm identity. As the layer again progresses into winter, it exposes its interfor buoyancy structure to the atmosphere, and warm central waters reemerge (Figs. IV.7 and 9)。 In Fig. IV.11, we plot a springtime XBT section taken across a warm core Ring which shows evidences of deep central wintertime mixing and weak surface temperature gradients.

Conversely, a Slope Water mixed layer moving into the Sargasso Sea is heated and develops a summertime thermocline. Subsequent evolution of the mixed zone is largely confined to a layer of thickness $h_{0}$ (see Fig. IV.10). In Fig. IV.12, we plot an XBT section taken across cold core Ring Bob on which has been drawn a subjective estimate of the mixed layer depth. While there is a suggestion that the mixed layer is shallower at Ring center, one questions whether the difference is significant. Still, in contrast to the warm Ring, cold Ring mixed layers are no deeper than those of the Sargasso. After being lost in the summer, the contrasts in SST across a cold Ring do not reappear because the surface heat flux mixes into layers of similar depth. Only at the end of winter does the unique structure of the core mixed layers appear. Note that the observations of winter mixed layer depth are 


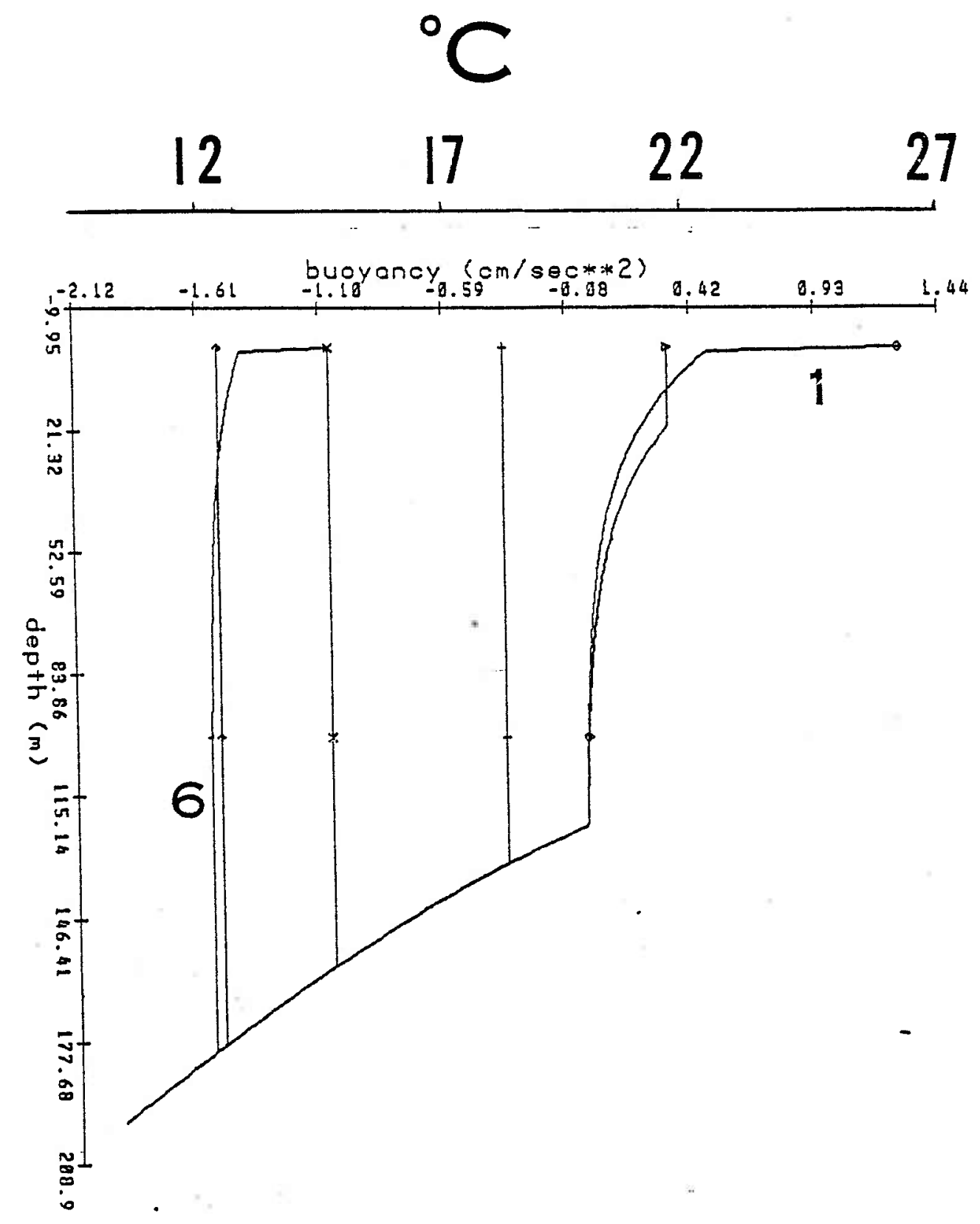

Figure IV.9. Lower Layer buoyancy Traces from a Warm Ring

Here we graph buoyancy against depth for depths greater than $30 \mathrm{~m}$ from an adjusting warm core Ring. The time interval between traces is two months and the first trace corresponds to July 1 . The relevant feature of the mixed layer buoyancy evolution concerns the deep, convectively driven mixing, which forms unusually thick layers thoughout the year. Also note the deep radiative heating. 


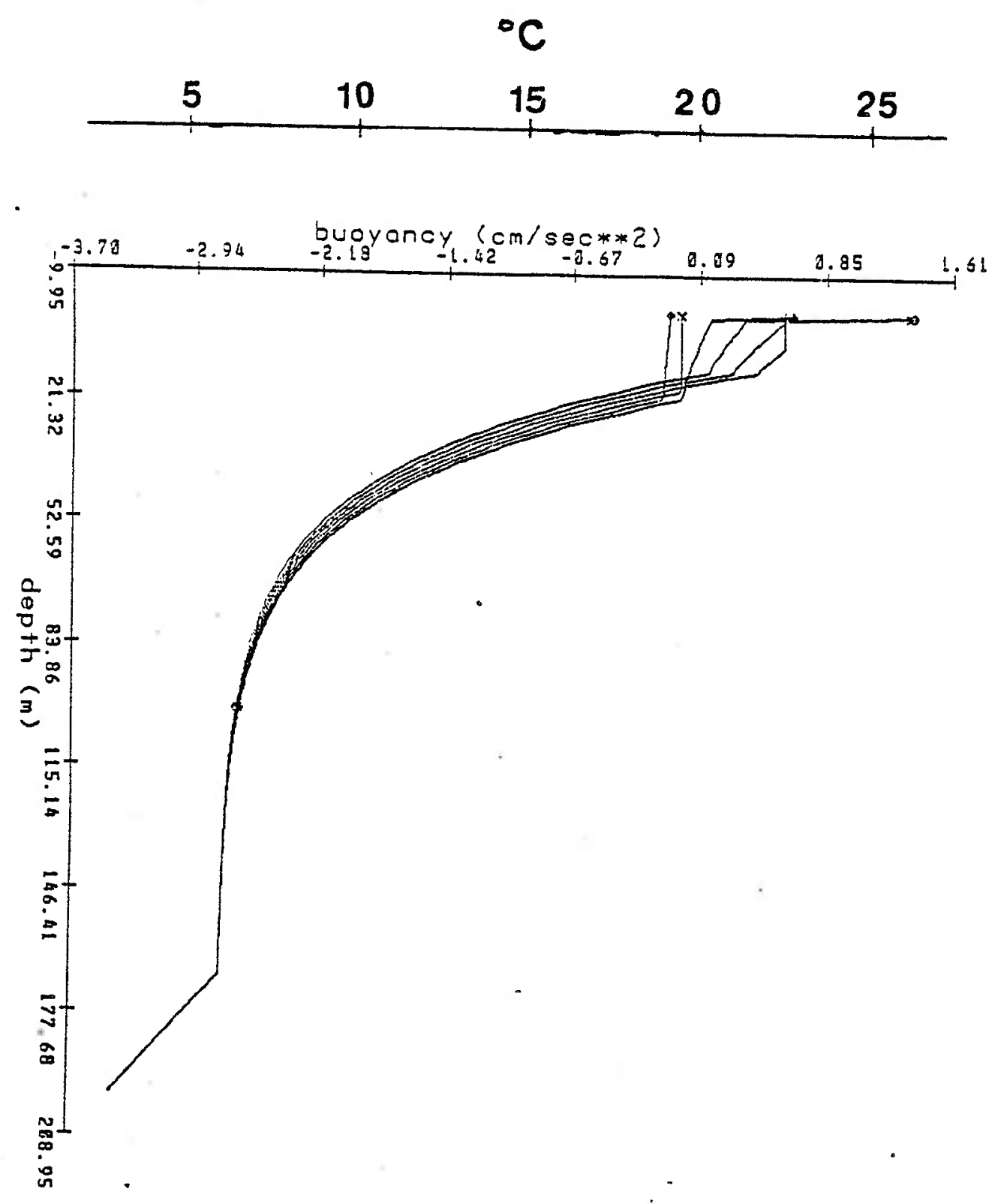

Figure IV. 10. Lower Layer Buoyancy Traces from a Cold Core Ring Here we graph buoyancy against depth for depths greater than $30 \mathrm{~m}$
from an evolving cold core surface layer. The time interval between
each corresponds to two each corresponds to two months, and the first corresponds to July 1 . because the Sargasso atmosphere 


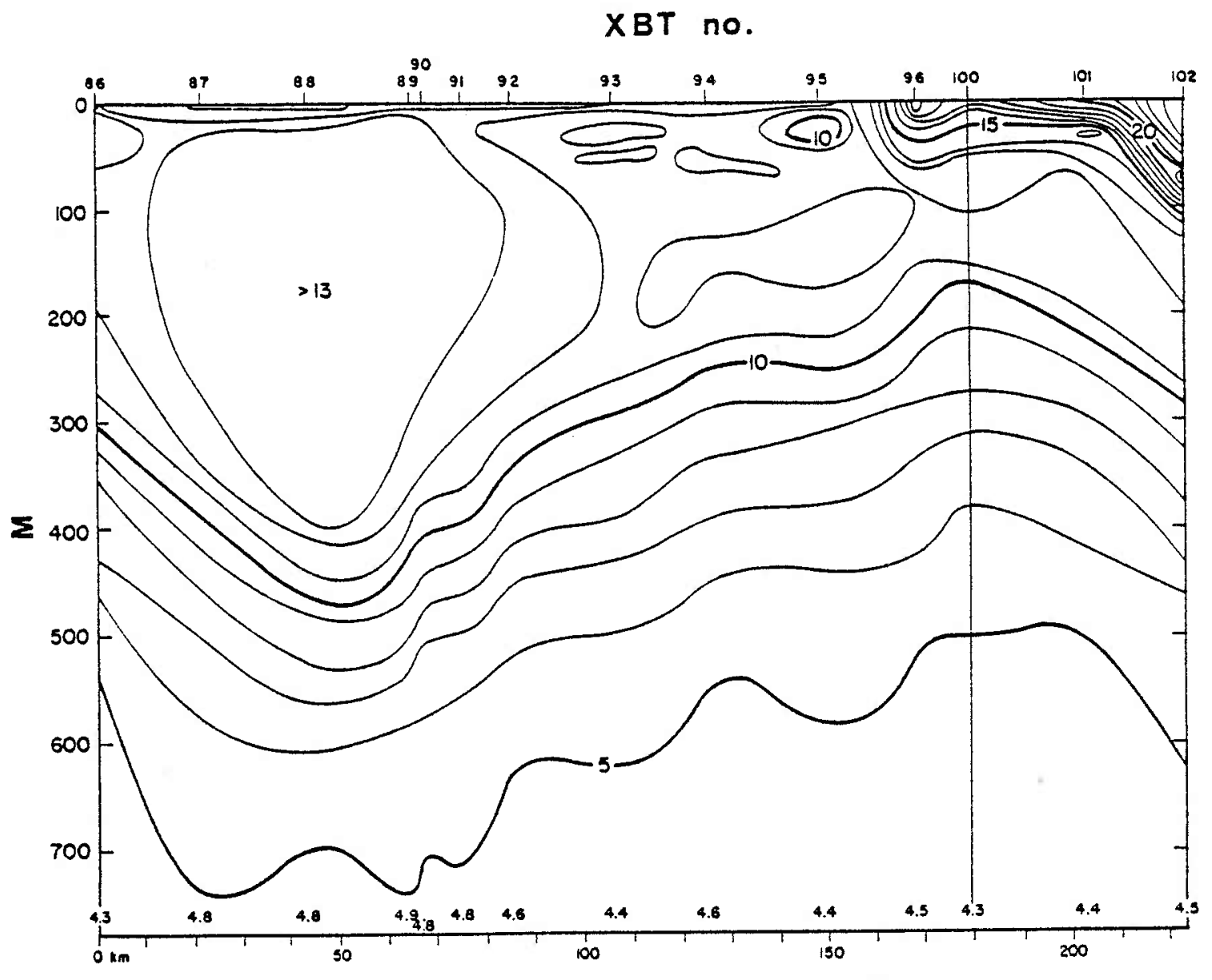

Figure IV.11. An XBT Transect of a Warm Core Ring

This transect, taken in the spring, shows many of the characteristics of the solutions we have been computing. Note that the core waters are well mixed to depths of $O(300 \mathrm{~m})$, and have been capped over by early springtime thermocline reformation. This data was obtained by Dr. Terrence Joyce, and is reproduced here by his kind permission. 


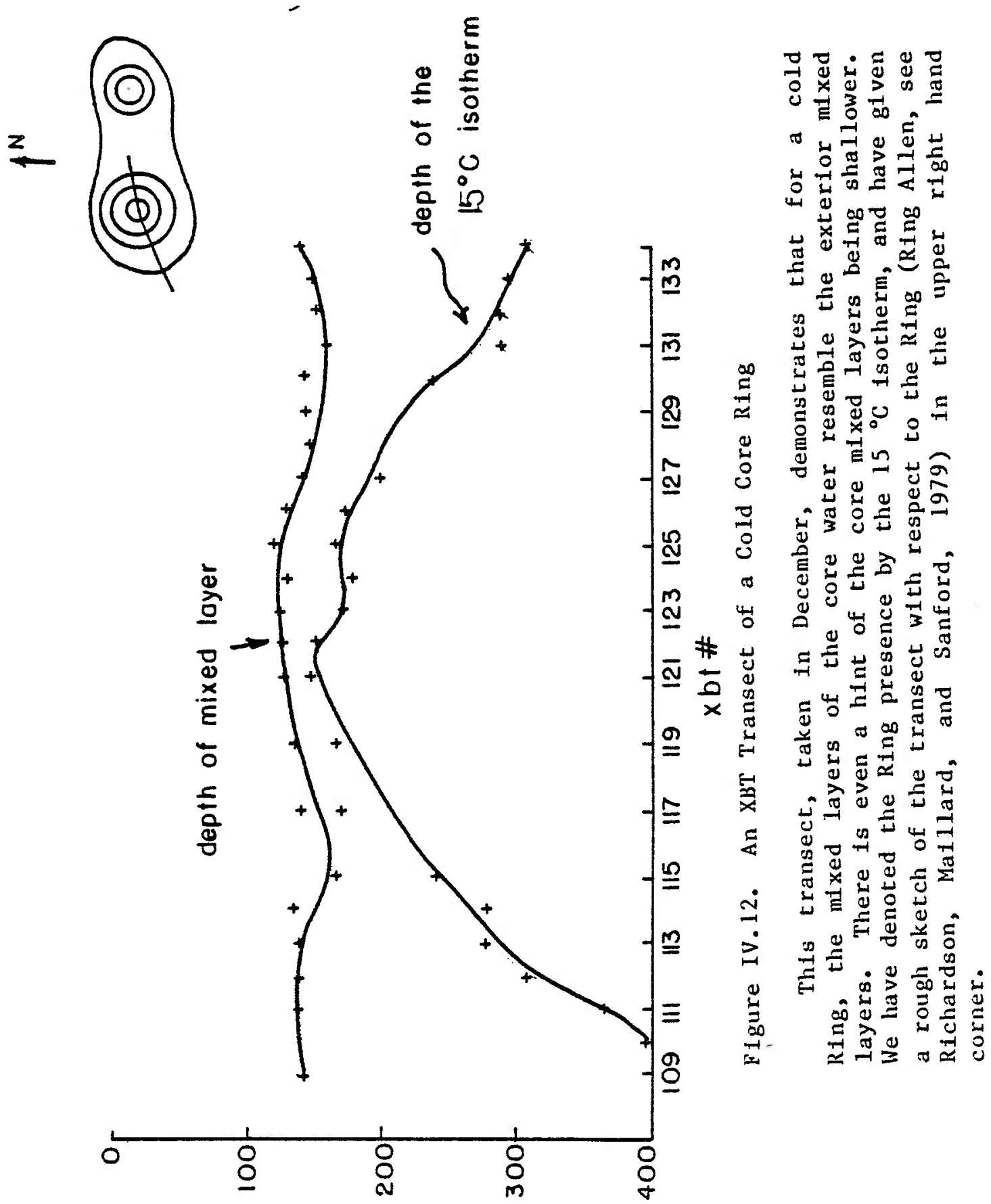

(U) $41 d \partial p$ 
roughly three times those of the model, which neglects intermediate layer mixing. Although $h$ is not well represented in late winter, the agreement improves in other seasons and therefore the SST behavior we have computed is probably insensitive to this.

With regards to the effects of differing formation dates, a series of experiments were conducted in which the Ring was 'formed' in different seasons. After a short adaptation, SST developed in the manner just described. This is in agreement with the above arguments which stress the contrasts in the air temperatures of the host and parent regions. Regardless of formation dates, within a year warm Ring cores will cool into very deep mixed layers and cold Ring cores will be heated and develop mixed layer depths of thickness $h_{0}$.

Finally note that there is a difference in the time scale of approach to the limit cycle states; warm Rings adjust much more quickly to the atmospheric forcing (on times of $O(2$ years)) than do the cold Rings (which take $O(10$ years $))$. The reason for this is that the intermediate layer is an integral part of the structure of the limit cycle mixed layer, and the mechanism of approach to its final state is governed by the loss or gain of heat. If the adjusting layer is being heated, the buoyancy at depth is increasing towards its limit cycle value. The excess heat necessary to do this comes from radiation, which at depths of, say, $150 \mathrm{~m}$ is a comparatively weak heat source and the layer requires several years to adjust (see Fig. IV.10). If the intermediate layer is being cooled, the loss of heat at depth is due to wintertime mixed layer cooling. Thus, the buoyancy structure is altered 
by turbulent heat transport, which is stronger than radiation, and the time scales of the evolution are the order of a few years. It is for this reason that we see a more rapid adjustment to the external forcing by warm pools than cold in Figs. IV.7 and 8, where the initially greater wintertime anomily of the anti-cyclonic eddy disappears at a faster rate than the cold anomaly. Thus, cold anomalies are more stable and long-lived than their warm counterparts. Ring lifetimes are $O(1 \mathrm{yr})$ owing to their interactions with the Gulf Stream, and therefore this is not an important process to Ring-SST evolution. We mention it because of possible applications to longer-lived SST anomalies in the general circulation.

IV.e Summary-

The evolution of the annual mixed layer was captured in a set of simple one-dimensional equations which were used to investigate the sea surface response of warm and cold Rings. We hypothesized that the important effect of the Ring was to transport water between the Slope and the Sargasso. The heat budgets of the core mixed layers were computed in terms of local air-sea exchange, and compared to the background SST cycles of the host Region. The resulting buoyancy-depth profiles and temperature contrasts compared well with data.

For warm core Rings, the important facet of the slope diabatic forcing was that it was on the average cooler than the temperatures which had built the structure of the core. As the Sargasso layers evolved, they developed unusually deep layers which had the effect of 
impeding the decrease in sea surface temperature. For cold core Rings, it was noted that the core mixed layers remained shallow, and therefore the temperature contrasts across the Ring were decreased in magnitude, because the Sargasso air temperatures were warm.

From Fig. IV.11 and these results, it is apparent that wintertime mixed layer development in a warm core Ring is a jor event. In the present calculation, we have neglected the dynamic effects of the adjusting buoyancy field on the Ring, and while the results are suggestive of Ring thermodynamics, it is clear that further modeling will require an active Ring. For example, the buoyancy traces contained in Figs. IV. 4 and 9 indicate a loss of dynamic height. With respect to 200 decibars, the pressure head of the core over the flank decreases by roughly 20 dynamic centimeters from July to February, resulting in a decrease of $30 \mathrm{~cm} / \mathrm{sec}$ in geostrophic velocity. Wintertime mixing carries out a thermodynamic spin-down of the Ring because the act of cooling the intermediate layer removes the depression in the upper layer 1sotherms. While the overall annual SST cycle will probably not change because of the inclusion of an evolving Ring, we can expect the Ring life cycle to be modified by the inclusion of the mixed layer.

The simplicity of the annual model suggests that the results may extend beyond the scope of the present problem. Consider the implications for the interpretation of satellite infra-red images. On the basis of the present chapter, we would interpret a warm pool, regardless of whether it is associated with a Ring, as indicating the presence of a deep, warm buoyancy profile. With historical information 
about geographical variations in density structure, we could even make some intelligent guesses as to its origin. Cold anomalies are not indicative of deep structure, although from an observational point of view, they are short-lived. Therefore, any observed cold pools are probably the result of a recent event. From large scale infra-red maps, we can search for similar temperatures and make an objective statement about the origin of the cold pool.

Also, we agree with Simpson and Dickey (1981) about the importance of penetrative radiation in the upper layers. In the present model, most of the intermediate layer restratification occurs by radiation, rather than by any process resembling detrainment (as occurs in turbulent erosion models). Also, solar irradiance is observed to operate in the intermediate zones of the real ocean in much the same manner as in the present model (see Appendix A.IV). Given the dependence of SST on intermediate layer buoyancy structure, we recommend that future models properly account for the vertical distribution of radiation.

Note, we have not considered geographical variations in optical properties, such as the attenuation coefficients, of the water in Rings. Richardson (1980) has reported that upon entering cold core Ring 'Bob', the water color changed and even the odor in the air developed a Slope Water character. With respect to Sargasso water, Slope water is 'dirty' and full of aquatic microorganisms and therefore the solar irradiation does not penetrate as deep in Slope water as in Sargasso water. Cold Rings are frequently observed in the sumertime to be a few 
tenths of a degree $C$ warmer than the surrounding Sargasso Water (Vastano, Schmitz, and Hagan, 1980); in other words, cold core Rings temporarily become warm core Rings. It is interesting to speculate that this is a manifestation of a more efficient absorption of radiant energy by the murky Slope Water mixed layers; whether this is a valid explanation remains to be seen. 


\section{Appendix A.IV A Bulk Mixed Layer Mode1-}

\section{A.IV.a The Equations and the Forcing Functions-}

The one-dimensional mixed layer model we shall employ is that originally discussed by Pollard, Rhines, and Thompson (1973, hereafter PRT) and cast into operational form by Thompson (1974, 1976). The three dimensional momentum and heat equations were derived in Chapter II; their one-dimensional forms are:

$$
\begin{array}{cl}
h b_{t}=\left(b_{i}-b\right) h_{t}-\tilde{\beta}\left(b-b_{a}\right)+B_{o s}-B_{0}(-h), & \text { a. } \\
(h u)_{t}-f_{o v h}=\tau_{x}, & \text { b. Eq. A.IV.1 } \\
(h v)_{t}+f_{o u h}=\tau_{y}, & \text { c. } \\
\left(b-b_{1}\right) h=(u 2+v 2), \text { and } & \text { d. } \\
\text { bit }=\text { Boz, } & \text { e. }
\end{array}
$$

where the meaning of each symbol is listed in Table IV.1. Eqs. A.IV.1.a-e allow the mixed layer to entrain in two different ways, one wind driven and one buoyantly driven. First, inertial oscillations, caused by fluctuations in $\tau$, produce large shears at the base of the mixed layer, and Eq. A.IV.d is the condition for the flow at the interface to be marginally stable. Further acceleration of the flow produces turbulence, which mixes the fluid under the interface up into the layer. Second, wintertime cooling at the surface causes convection which cools the layer and deepens the interface to cooler, denser depths. Note Eqs. A.IV.1 do not allow for penetrative convection, so that a jump in density at the mixed layer base can be maintained only through the dynamic instability of the shear flow. Little evidence of penetrative convection has been observed in the mid ocean and 
model-data agreement improves if it is neglected (Gill and Turner, 1976).

Deszoeke and Rhines (1976) discuss the solution to the mixed layer equations using an energy closure of the form:

$$
1 / 2 \mathrm{e}\left(n u_{*} 2+\mathrm{N} 2 \mathrm{~h}^{2} / 2-(\underline{\mathrm{u}}-\underline{\underline{u}} i)^{2}\right)=\mathrm{mu}_{*}{ }^{3} \quad \text { Eq. A.IV.2 }
$$

a so-called 'turbulent erosion model' closure. They assumed a constant wind stress, chose $h=0$ inftially, and neglected diabatic effects, and found that, as the mixed layer evolved, Eq. A.IV.2 described four different balances between the possible energy sources and entrainment. The first two were associated with the rapid deepening of an initially unmixed surface layer, the third with deepening due to shear instability such as is governed by Eq. A.IV.1.d, and the fourth with a balance between wind wave breaking and entrainment. Although the entrainment rate of fluid in the last stage was small compared with that of the first three stages, they suggested that this balance described the long-term deepening of the mixed layer. Here our view will be that heating and cooling, along with shear induced Kelvin-Helmholtz instabilities dominate the energy balance, which is consistent in a layer which never completely restratifies $(h \neq 0)$ and is subjected to a variable wind stress.

Finally, a test of the present model using data from Ocean Weather Station ' $N$ ' returned a $98 \%$ correlation between predicted and observed sea surface temperature (Thompson, 1976). 
Meteorological and Solar Data-

For the Thompson model to operate properly, detailed information about the meteorological forcing is required. In this section, we discuss the data which we used.

Winds-

Spectra of wind velocity from various ocean weather stations have been reviewed recently by Muller (1981) who arrived at model spectra for both zonal and meridional wind speed. In a manner similar to Thompson (1973) and Liu and Thompson (1976), we converted those spectra into time series of wind speed. First, the model spectra were sampled at a finite number of frequencies. Next the square roots of the spectral values were multiplied by randomly generated phases:

$$
\begin{aligned}
\hat{u}_{I}(\sigma)+ & \dot{i} \hat{u}(\sigma)=\hat{u}(\sigma)=|E(\sigma)|^{1 / 2} e^{i \eta}, \text { where } \\
\langle e i \hat{\eta}\rangle & =0,
\end{aligned}
$$

thus composing a complex vector of Fourier amplitudes. The vector was subsequently fast Fourier transformed to physical space, producing a record of wind speed. By varying the interval and the domain with which we sampled the spectra, time series of wind speed differing in duration and frequency of observation were obtained. The data so generated, however, suffers from the defect that the wind speeds are not governed by a Gaussian parent population, because the magnitudes of the Fourier coefficients have not been randomized. Thus, it is doubtful if our artificial data represents data which would ever be realized in nature (Wunsch, personal communication). On the other hand, we did obtain a gap free data set, which contained the proper amount of energy. 
To the stochastic series determined by the spectra, we added mean winds, tides, and seasonal signals. Mean wind speed was taken to be 7 $\mathrm{m} / \mathrm{sec}$ to the east. The tides and annual cycle were changed from experiment to experiment; their variation seemed not to effect the mixed layer and in the results here, the only retained deterministic component was the mean wind. Finally, stress was computed according to:

$$
\underline{\tau}=\left.\hat{\mathrm{C}}_{\mathrm{d}}\right|_{\underline{u}_{\mathrm{a}} \mid \underline{u}_{\mathrm{a}} \text {, }}
$$

a sample time series of which is contained in F1g. A.IV.1.a.

\section{Air Temperature-}

At low frequencies, the fluctuations in temperature were made coherent with the meridional wind, i.e. winds from the south were assumed to convect warm air and those from the north, cold (Muller, 1981). The magnitudes of the temperature fluctuations were made linearly proportional to the wind speed. At higher frequencies, the coherence with north-south winds was decreased and a purely white noise component was added. To the random temperature series we added a yearly mean, an annual cycle, and a weaker $\left(\approx .5^{\circ} \mathrm{C}\right)$ daily signal. An example of a model temperature series is included in Fig. A.IV.1.b; note that there are energetic fluctuations but that the record is dominated by the annual variation.

Solar Heating-

In character, solar radiation is different from surface heat exchange in that the radiant energy can penetrate into the water 

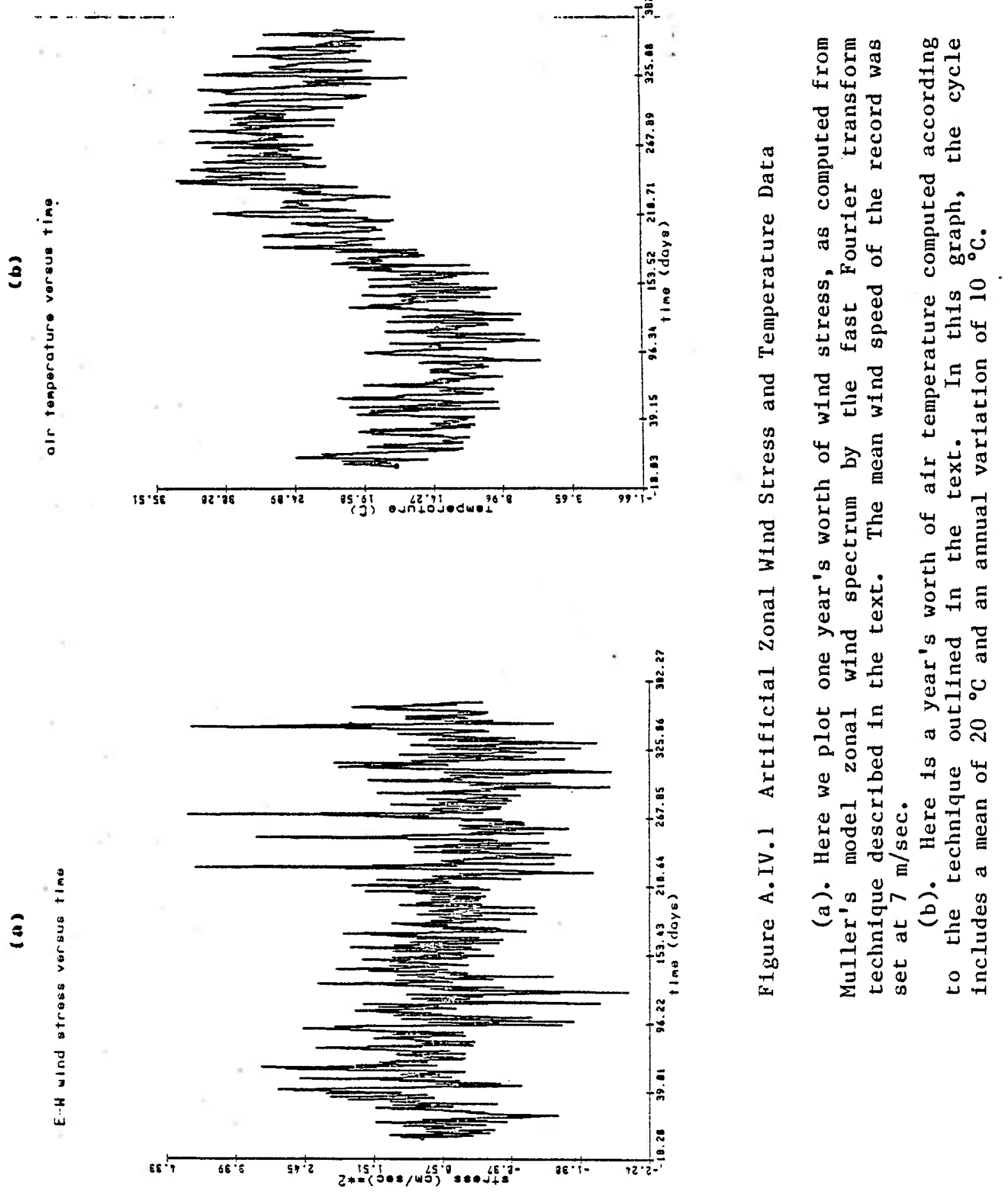

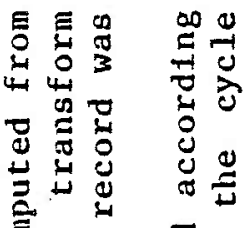

预导

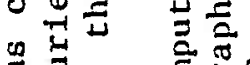

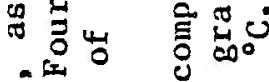

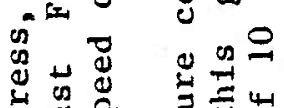

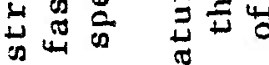

モ

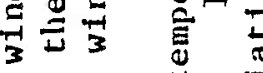

茨各政

$=$ 至

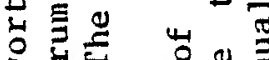

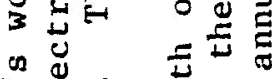

on

प्रें 这 $z=$

उन

तण क

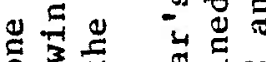

궁

o

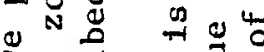

$\rightarrow$ 讨

4 o 0

㞹

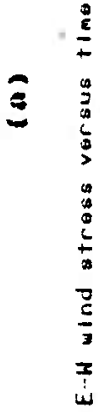

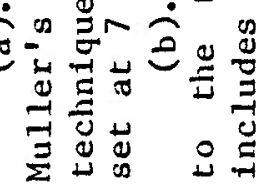


column and act as an internal heat source. As a parametrization of solar irradiance, we employed:

$$
B_{O}(z)=M_{0} V(t)\left(\operatorname{Re}^{z / \lambda} 1+(1-R) e^{z / \lambda 2}\right)
$$

(Krauss, 1972; Paulson and Simpson, 1977). The values assigned to the constants are listed in Table IV.1. Note that the e-folding depth $\lambda_{1}$ is very shallow $(35 \mathrm{~cm})$. Operationally, the radiation from this exponential was obsorbed within the first meter. The e-folding depth $\lambda_{2}$ is typically much greater; we used 25 mo $\lambda_{2}$ is, however, a function of the biological and sediment content of the water and can vary from 10 m In coastal water to $33 \mathrm{~m}$ in the open ocean (Niiler, 1977). The magnitude of solar radiation is set by both the zenith angle and the hour angle of the sun, and in the present model was computed according to:

$$
\begin{aligned}
& V(t)=e^{-.13 / \cos (\phi)} \text {; if } \cos (\phi)>0 \\
& V(t)=0 \text { otherwise, }
\end{aligned}
$$

where:

$$
\cos (\phi)=-\cos (\theta) \cos (\psi) \cos (\Omega t)+\sin (\dot{\theta}) \sin (4),
$$

with 4 the latitude, $\Omega=2 \pi /(1$ day $)$, and $\theta$ the zenith angle. $\theta$ was computed according to:

$$
\theta=23^{\circ} \cos (2 \pi *(355-(\text { julian day })) / 365)
$$

(Thompson, 1974). Note that the above formula extinguishes the solar radiation for roughly half of every day.

The amount of energy which enters the intermediate layer by penetrative radiation is small; however, it is the exclusive (positive) heat flux in that layer for periods up to 11 months. The importance of solar irradiance in the annual mixed layer structure was demonstrated in the main body of the chapter. 


\section{A.IV.b Initial Experiments with the Thompson Model-}

We conducted a series of mixed layer simulations using the numerical technique documented in Thompson (1976) in order to illustrate the processes which govern the annual mixed layer cycle. In all experiments reported here, we used a time step of 80 minutes and a vertical grid spacing of one meter.

In Fig. A.IV.2. a we display SST and atmospheric temperature against time from a four year mixed layer simulation. This graph demonstrates the cyclical ability of the model. In Fig. A.IV.1.b, we show a similar graph as determined by the Marine Climatic Atlas. Note that several features of the mixed layer in the data are well reproduced in the model results. For example, SST extrema lag atmospheric temperature extrema by about 30 days. Secondly, we note that at almost all times of the year, sea surface temperature is actually greater than atmospheric temperature, which indicates that the heat flux due to sensible and evaporative exchange is directed out of the mixed layer. This indicates the importance of the solar radiation, for it provides the heat necessary to warm the mixed layer. Finally we remark on the asymmetric annual sea surface temperature signal, first discussed by Warren (1973) and Gill and Turner (1976).

In Fig. IV.3, shown in the main body of the chapter, we plotted buoyancy profiles as computed by this model. They correspond to the SST graph of Fig. A.IV.2.a, and should be compared to Fig. A.IV.3, where we include some XBT traces from the Slope Water (Dr. Peter Wiebe, personal 

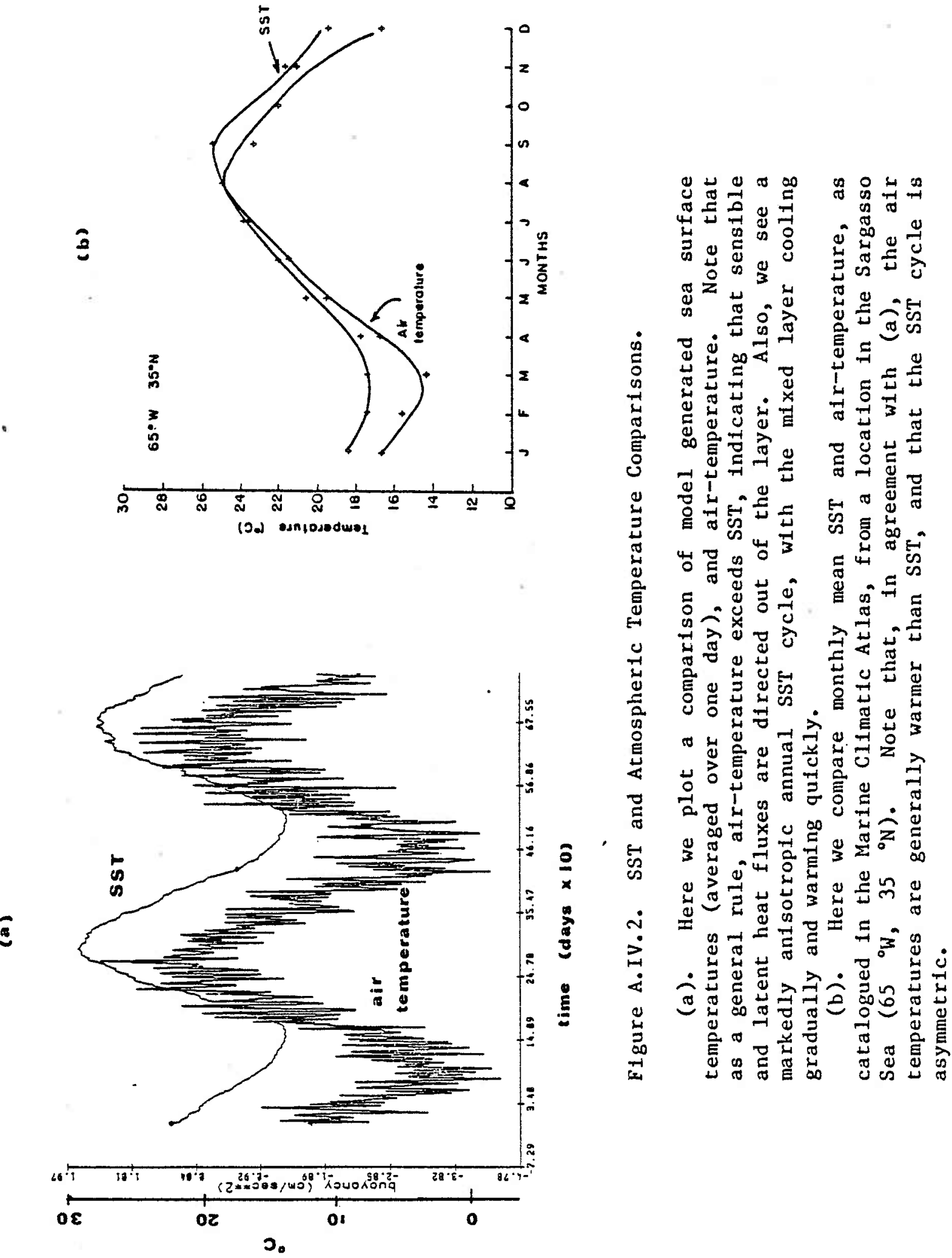

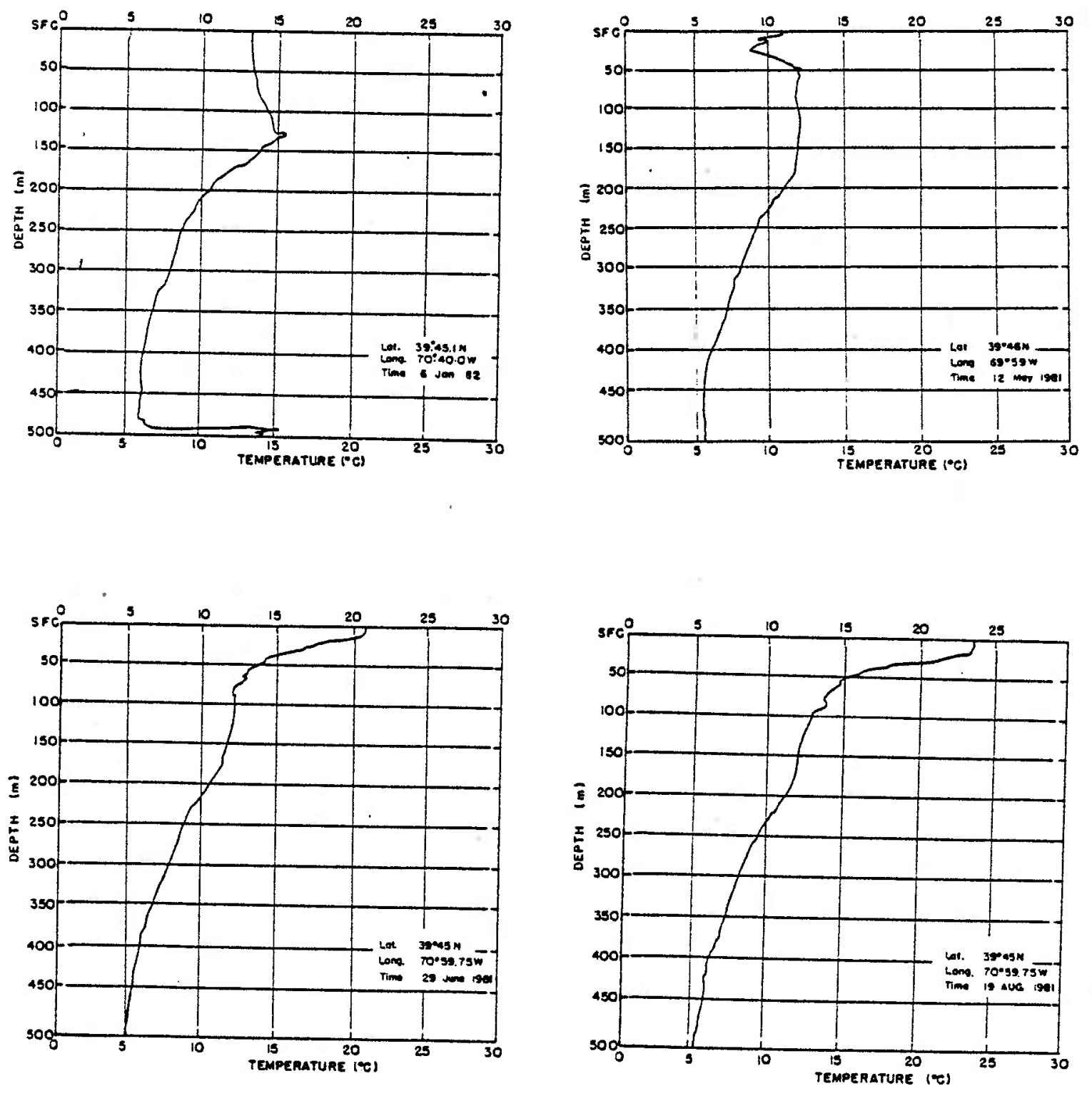

Figure A.IV.3. Temperature Traces from the Slope water

Here are several temperature traces, taken at various times of the year, from the Slope water. In agreement with the model output in Fig. IV.3, we see shallow summer mixed layers, and evidences of both deep winter-time mixing and penetrative radiation. This data was obtained by Dr. P. Wiebe and is reproduced here by his kind permission. 
communication) obtained at various times of the year. Clearly a strong vertical gradient of density develops in Fig. A.IV.3 in the upper $50 \mathrm{~m}$ as the layer progresses through the summer. We also see evidences of penetrative radiation. Comparing the 50 meter depths in Fig. A.IV.4.b and $d$ shows an increase in temperature of $3{ }^{\circ} \mathrm{C}$. In view of the almost perfect match of the very deep $(0(500 \mathrm{~m}))$ thermal structure of these traces, it is unlikely that the difference is due to advection. Because of the strong density gradient, it is equally unlikely that we can account for the additional heat at $50 \mathrm{~m}$ in terms of surface exchange, leaving radiation as the most probable explanation. Finally, the shape of the intermediate layer temperature profile in the data is similar to that of the model. 
Appendix B.IV The Sensitivity of Mixed Layer Development to

\section{Buoyancy Flux-}

The behavior of the mixed layer depends critically on the sense of the heat flux, or the season. In the present appendix, we construct simple analytic models with a view towards understanding the distinctions between spring and fall mixed layers.

The Reformation of the Thermocline-

The depth over which incoming heat is mixed changes abruptly in early spring (see Thompson, 1974). The first problem concerns the manner by which the new thermocline is established.

Note from the traces in Fig. IV. 3 that the late-winter/early-spring surface waters are characterized by a deep uniform layer. During perlods of calm $(\underline{\tau}=0)$ in the early spring, surface exchange inserts buoyancy into the upper few meters, which is subsequently mixed by the resident turbulence (due to wave breaking, and/or Langmuir circulation, see Fig. B.IV,1). With the onset of a wind event, inertial oscillations are generated and the layer commences shear-induced deepening, as in the problem of Pollard, Rhines, and Thompson (1973, hereafter PRT). We can solve the momentum equations, Eq. A.IV.1.a and b, to obtain:

$$
u^{2}+v^{2}=\frac{2 \tau_{x}^{2}}{f_{0}^{2} h^{2}}\left(1-\cos \left(f_{0} t\right)\right) \cdot \quad E q \cdot B \cdot I V \cdot 1
$$




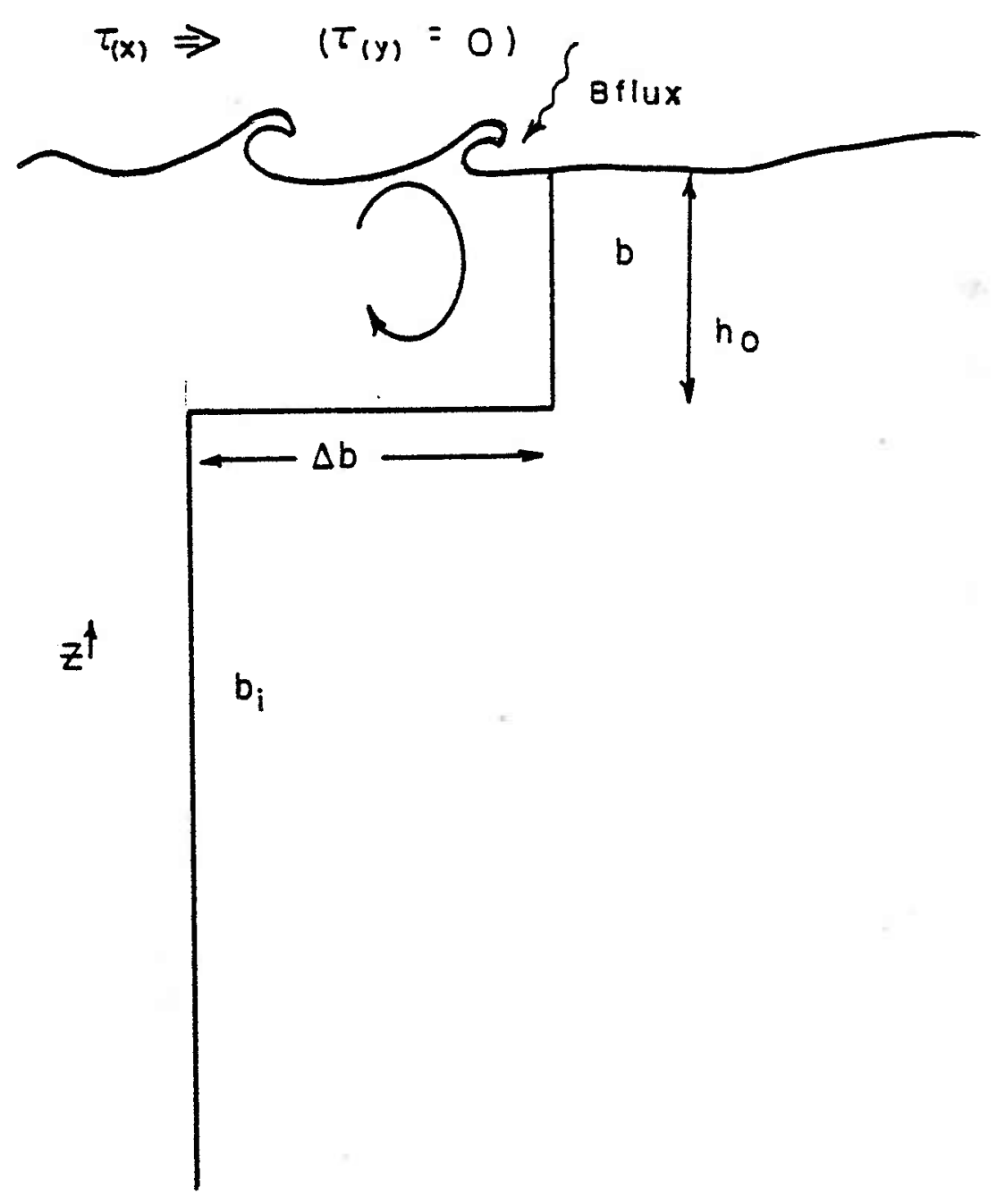

Figure B.IV.1. Schematic of Thermocline Reformation

In early spring, the intermediate layer has been well-mixed by winter time convection. During periods of calm, heat is injected and mixed in the upper few meters. With the onset of a wind event, shear-induced deepening mixes the heat downward and creates a new thermocline. 
Neglecting further buoyancy flux (equivalent to the assumption that the adjustment to the wind will be rapid), the heat equation yields:

$$
(\Delta b) h_{0}=\left(b-b_{i}\right) h \quad \text { Eq. B.IV.2 }
$$

where $\Delta b$ and ho are the inftal buoyancy jump and mixed layer depth respectively. From Eq. A.IV.1.d:

$$
h^{2}=2 \tau_{x}^{2}\left(1-\cos \left(f_{0} t\right)\right) /\left(f_{0}^{2}(\Delta b) h_{0}\right) \text {. Eq. B.IV. } 3
$$

Eq. B.IV. 3 is valid so long as $h_{t}>0$, which is true up to time;

$$
f_{o t}=T \text {, }
$$

after which the inertial oscillations decelerate and the mixed layer remains at its maximum depth, given by:

$$
h_{m}=2 E_{x} /\left(f_{0}\left((\Delta b) h_{0}\right)^{1 / 2}\right) . \quad \quad E q \cdot \text { B.IV. } 4
$$

$(\Delta b) h_{0}$ is proportional to the anomaly of heat in the mixed layer prior to the onset of the wind. A heat flux due to an $0\left(5^{\circ} \mathrm{C}\right)$ air-sea temperature difference, operating for 10 hours, produces $a(\Delta b) h_{0}$ of 36 $\mathrm{cm}^{2} / \mathrm{sec}^{2}$. Subsequently, a 1 dyne $/ \mathrm{cm}^{2}$ wind stress will produce a 30 meter mixed layer. The interesting point of this result is that it is the heat stored in the upper few meters by air-sea exchange which determines the final depth of the mixed layer. In PRT, the slope of the interfor buoyancy profile played the same role. Clearly, the energy in the inertial oscillations is used to mix the buoyant water down into the wintertime profile. Several such events produce a warm layer which covers over the deep, well-mixed buoyancy; deeper mixing is then energetically resisted by the gravitational stability of the stratification. From Eq. A.IV.1.d, a $1 \mathrm{~cm} / \mathrm{sec}^{2}$ buoyancy jump at a depth of 30 meters requires a wind stress of $O\left(6 \mathrm{dynes} / \mathrm{cm}^{2}\right)$ to reach criticality and entrain, hence the large density gradient of summertime acts as a barrier to shear-induced entrainment, and the fall 


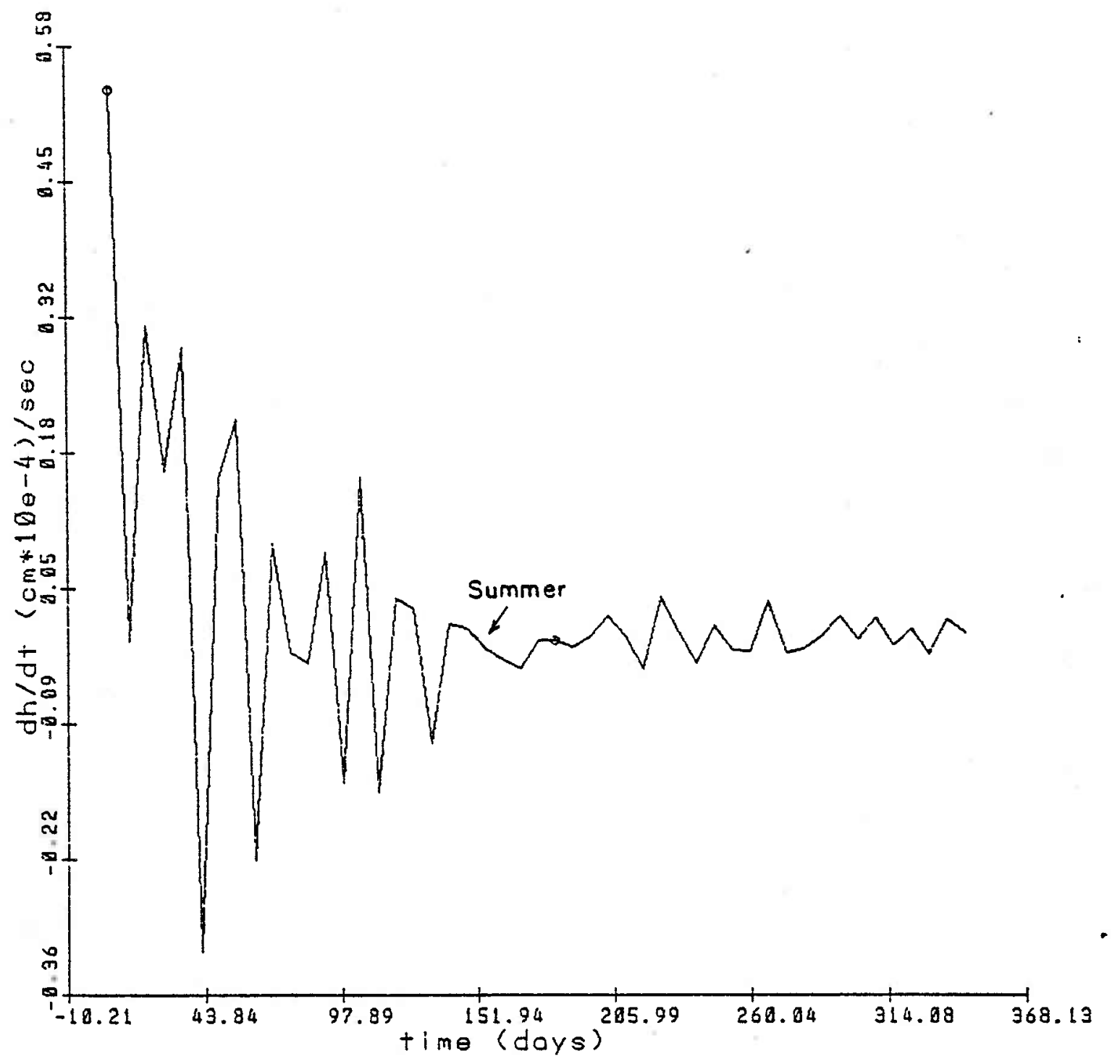

Figure B.IV.2. $h_{t}$ Versus Time.

Here we plot numerical measures of ht (seven day averages) as determined by Thompson's model. Note that with the onset of summer, the magnitude of $h_{t}$ decreases markedly, Indicating that the seasonal thermocline acts as a barrier to mixing. 
cooling season season must erode it before the mixed layer can extend far into the fluid. This is further confirmed by Fig. B.IV.2, which shows a numerical measure of $h_{t}$, where $h$ is a several day average of the mixed layer depth, from a simulation using Thompson's model. Notice that the magnitude of the fluctuations decreases markedly during the summer.

Wintertime Mixed Layers-

If the sea surface is cooled, the mixed layer can deepen efficiently. We note from Fig. IV.3 that a deep mixed layer joins without a discontinuity, $b=b i$, onto the intermediate layer buoyancy, which indicates that the heat budget of the layer is dominated by the surface fluxes (see Eq. IV.1.C). In the following problem, we shall investigate these features of the wintertime mixed layer.

The lack of a density step may be explained from an energetic point of view. From Eq. IV.I.d, the difference in buoyancy at the mixed layer interface is given by:

$$
\left(b-b_{i}\right)=\left(u^{2}+v^{2}\right) / h=2 \tau_{x}^{2}\left(1-\cos \left(f_{o t}\right)\right) / f_{o h^{3}} \text {. Eq. B.IV. } 5
$$

For a two dyne $/ \mathrm{cm}^{2}$ wind stress acting on a $25 \mathrm{~m}$ layer, the buoyancy jump, $b-b_{i}$, is $.1 \mathrm{~cm} / \mathrm{sec}^{2}$ and falls off as $h^{3}$. It is very difficult for thick layers to maintain sizeable density steps in buoyancy structure, due to the depth over which the momentum is mixed. 
Now consider the relative importance of entrainment heat flux with respect to air-sea exchange. Suppose the layer is subject to a constant negative buoyancy flux $\mathrm{H}_{\mathrm{O}}$ and the density structure of Fig. B.IV.3. In the same manner as the thermocline reformation problem, we arrive at:

$$
\begin{aligned}
2 h^{2}= & \left(\left(h_{0}^{2}-\frac{2 \mathrm{H}_{0} t}{\Gamma}\right)+\left[\left(h_{0}^{2}-\frac{2 \mathrm{H}_{0} t}{\Gamma}\right)^{2}+\right.\right. \\
& \left.+\frac{16 \frac{2}{\tau_{x}^{2}\left(1-\cos \left(f_{0} t\right)\right)}}{\left(\Gamma \mathrm{f}_{0}^{2}\right)}\right]^{1 / 2},
\end{aligned}
$$

where ho is the initial mixed layer depth. Again, this solution remains valid so long as $h_{t}>0$, or until time:

$$
\text { fotc }=\pi
$$

Up to that time, we see:

$$
2 \mathrm{H}_{\mathrm{ot}} /\left(\mathrm{ho}_{\mathrm{O}}^{2} \mathrm{\Gamma}\right)=20 \times 10^{4} /\left(25 \times 10^{6}\right)=.02 \ll 1 \text {, }
$$

and:

$$
16 \tau_{\mathrm{x}}{ }^{2} /\left(\mathrm{rf}_{\mathrm{O}}{ }^{2} \mathrm{ho}_{\mathrm{o}} 4\right)=32 / 625=.05<1
$$

where we have used $\tau_{x}=2$ dynes, ho $=50 \mathrm{~m}, \Gamma^{\top}=10^{-4} \mathrm{sec}^{-2}, t=\pi / \mathrm{f}_{0}$, and $H_{0}=10^{-3} \mathrm{~cm}^{2} / \mathrm{sec}^{3}$, which allows us to expand the square root. Hence:

$$
h^{2}=h_{0}^{2}-2 H_{0} t / \uparrow+4 \pi_{x}^{2}\left(1-\cos \left(f_{0} t\right)\right) /\left(\Gamma f_{0}{ }^{2} h_{0} 2\right)
$$

The entrainment rate is given by:

$$
h_{t}=-H_{0} /(r h)+2 \tau_{x^{2}} \sin \left(f_{\circ}\right) /\left(\Gamma f_{\circ} h_{0} 2 h\right), \quad \text { Eq. B.IV. } 6
$$




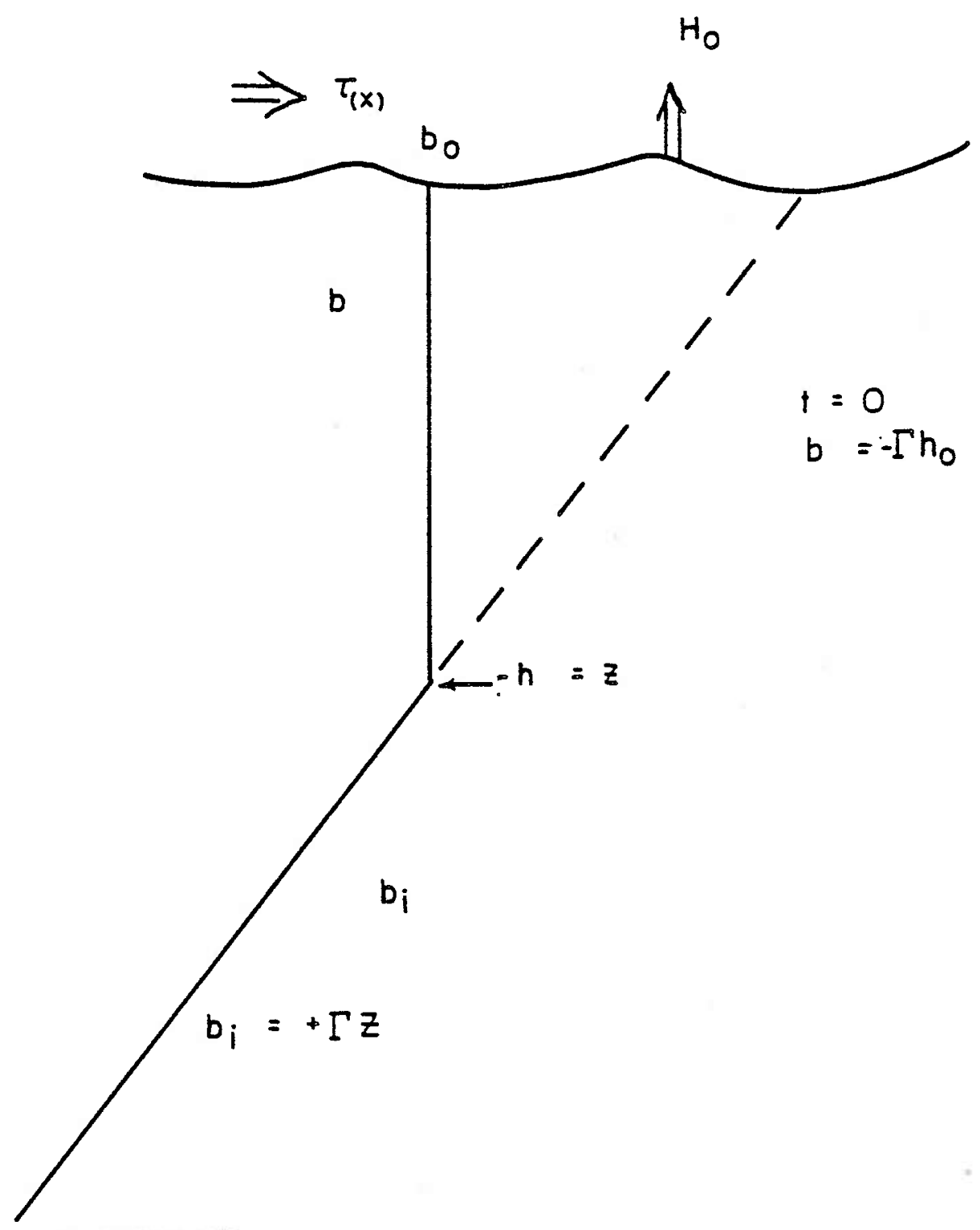

Figure B.IV.3. Schematic for Deep Mixed Layer Entrainment

We will consider the upper ocean to be composed initially of two layers, a well-mixed upper layer, of depth $h_{0}$, and a linearly stratified intermediate layer, such that $-\Gamma h_{0}=b_{0}$. We also assume that the atmosphere is drawing heat out of the mixed layer at a constant rate, and that the wind is being maintained at a constant value. 
therefore, the ratio of entrainment flux to surface flux is:

$$
\begin{aligned}
(\Delta b) h_{t} / H_{0} & =-2 \tau_{x}^{2}\left(1-\cos \left(f_{0} t\right) /\left(f_{0} 2 h^{4} \Gamma\right)+E q \cdot B . I V .7\right. \\
& +4 \tau_{x}^{4} \sin \left(f_{O} t\right)\left(1-\cos \left(f_{0} t\right)\right) /\left(f_{0} 3 r H_{0} h_{0} 2 h^{4}\right) .
\end{aligned}
$$

The orders of magnitude for the terms in Eq. B.IV.7 are:

$$
-2 \tau_{x^{2}}\left(1-\cos \left(f_{\circ} t\right)\right) /\left(f_{o} 2 h^{4} \Gamma\right)=16 / 625 \ll 1,
$$

and:

$$
\begin{gathered}
4 \tau_{x}{ }^{4} \sin \left(f_{\circ} t\right)\left(1-\cos \left(f_{\circ} t\right)\right) /\left(f_{\circ} 3 r^{3} B_{\circ} h_{0} h^{4}\right)= \\
\quad=64 / 1562.5 \ll 1,
\end{gathered}
$$

so the approximate heat equation governing the layer is:

$$
h b t=-\tilde{\beta}(b-b a)+B o s-B o(-h) . \quad \text { Eq. B.IV. } 8
$$


Appendix C.IV Verification of the Annual Mixed Layer Equations-

C.IV.a Choice of $\mathrm{h}_{0}-$

The solution to the summertime equations may be compared to data to specify $h_{0}$. The general solution for surface buoyancy during summer is: $b=A_{o e}-\bar{B}(t-t 1) / h o+C \cos (w t+\bar{L})+D \sin (\omega t+\bar{\omega})$, where:

$$
\begin{array}{ll}
A_{0}=b_{1}-\left(C \cos \left(\omega t_{1}+\pi\right)+D \sin \left(\omega t_{1}+\tau\right)\right), & \\
C=\tilde{\beta} 2 a_{1} /\left(\omega 2 h_{0}{ }^{2}+\overline{2}\right), & \text { Eq. C.IV.1 }
\end{array}
$$

and:

$$
\mathrm{D}=w \mathrm{ho} \tilde{\beta}_{\mathrm{a}} 1 /\left(w^{2} \mathrm{~h}_{0}{ }^{2}+\tilde{i} 2\right),
$$

where $b_{1}$ is the initial sea surface buoyancy at $t 1$, the onset of 'spring'. Eqs. C.IV.I are valid for $t 1<t<t 2$ where $t 2$ marks the transition from heating to cooling, i.e. the onset of 'fall'. Notice that a mixed layer 'relaxation time':

$$
t_{r l}=h_{0} / \tilde{\beta}
$$

emerges from the solution and defines the time necessary for the summer mixed layer to lose memory of the late winter surface buoyancy $b_{1}$.

The onset of fall, $t_{2}$, is defined by:

$$
-\tilde{\beta}(b-b a(t 2))+B f=0 \quad E q \cdot C . I V \cdot 2
$$

where we have used the short hand notation:

$$
B f=B a s(0)-B_{a}(-h) \text {. }
$$

Using Eq. IV.2 in Eq. C.IV.2 returns an implicit equation for $t_{2}$. 


$$
\begin{aligned}
A_{0 e}-\tilde{\beta}\left(t_{2}-t_{1}\right) / h_{0} & +C \cos \left(\omega t_{2}+\tau\right)+D \sin \left(\omega t_{2}+\bar{\sigma}\right)+ \\
& -a_{1} \cos \left(\omega t_{2}+\bar{\sigma}\right)=0 . \quad \text { Eq. C.IV.3 }
\end{aligned}
$$

Assuming that $t_{r 1}$ is short compared to $t_{2}-t_{1}$ :

$$
\exp \left(-\tilde{\beta}\left(t_{2}-t_{1}\right) / h_{0}\right)=0\left(\exp \left(-t_{h} t_{\text {season }} / t_{r}\right)<1,\right.
$$

and to the neglect of the exponential term:

$$
\tan ^{-1}\left(\left(a_{1}-C\right) / D\right)+n \pi=w t 2 . \quad E q \cdot C \cdot I V \cdot 4
$$

Evaluating the argument of the inverse tangent, we see that $t 2$ is independent of the magnitude of the diabatic forcing and depends only on system parameters.

$$
\tan ^{-1}\left(\omega h_{0} / \tilde{\beta}\right)=\omega t 2+n \pi \quad \text { Eq. C.IV.5 }
$$

Note from Fig. A.IV.2.b that the mixed layer maximum SST (necessarily occurring at $t 2$ ) lags the atmospheric maximum temperature by one month. From Eq. IV.2, maximum air-temperature occurs at $\Pi$, therefore the corresponding value of $t_{2}$ is $7 \pi / 6$, which in Eq. C.IV.5, returns a value of:

$$
\omega h_{0} / \tilde{\beta}=.6 \text {. }
$$

With $\tilde{\beta}=10-3 \mathrm{~cm} / \mathrm{sec}$ (Frankignoul, personal communication), and $\omega=$ $2 /\left(10^{7} \mathrm{sec}\right)$, we obtain a value for $h_{0}$ of $30 \mathrm{~m}$. It is this value which we have employed throughout the chapter.

\section{C.IV.b Validation-}

Using the analytical solution to the summertime mixed layer equations, we can estimate maximum sea surface temperature. Eq. C.IV.5 at t2 to the neglect of the exponential terms is:

$$
b_{\max }=(\sqrt{3} / 2) \tilde{\beta} 2 a_{1} /\left(\omega=2 h_{0}^{2}+\tilde{\beta} 2\right)+B f / \tilde{\beta}+a 2 \text { Eq. C.IV. } 6
$$

Using $h_{0}=30 \mathrm{~m}$ and a typical value for $B f$, we have:

$$
b_{\max }=a_{2}+.87 \mathrm{a} 1+.6 . \quad \text { Eq. C.IV. } 7
$$


The '.6' in Eq. C.IV.7 is due to radiative heating. As a test of Eqs. IV.1, we compared the maximum SST predicted by Eq. C.IV.7 with data. As an example, consider the Sargasso Sea air-temperature cycle, depicted in Fig. IV.1. The parameters describing the cycle are $a_{2}=0$ $\mathrm{cm} / \mathrm{sec}^{2}$, and $a_{1=1} \mathrm{~cm} / \mathrm{sec}^{2}$, and predict a maximum SST of 27.3 ${ }^{\circ} \mathrm{C}$. This is about $1{ }^{\circ} \mathrm{C}$ too warm. Several such checks at various locations were carried out, with the general result that predicted temperature is $0\left(.5^{\circ} \mathrm{C}\right)$ too warm, but nowhere grossly incorrect. In view of the very simple equations responsible for the prediction, the agreement was surprising and heartening.

Analogous analytical tests for the wintertime form of the equations were not found; however, in numerical solutions it was noted that relevant sea surface temperature ranges were well predicted, as were the dates of shift from winter to spring.

A second independent check of Eq. IV.I was made by direct comparison with the Thompson numerical model. In Fig. C.IV.1, we have plotted two atmospheric temperature cycles, one a smooth version of the other, which were used as the data sets for the comparison. The unsmoothed version was used to force the Thompson model, and its smoothed counterpart Eqs. IV.1. The comparison of predicted SST from the Thompson model, averaged over one day, and that from Eqs. IV.1 is displayed in Fig. C.IV.2; the agreement is very good. Similar comparisons between model mixed layer depths were equally encouraging. 


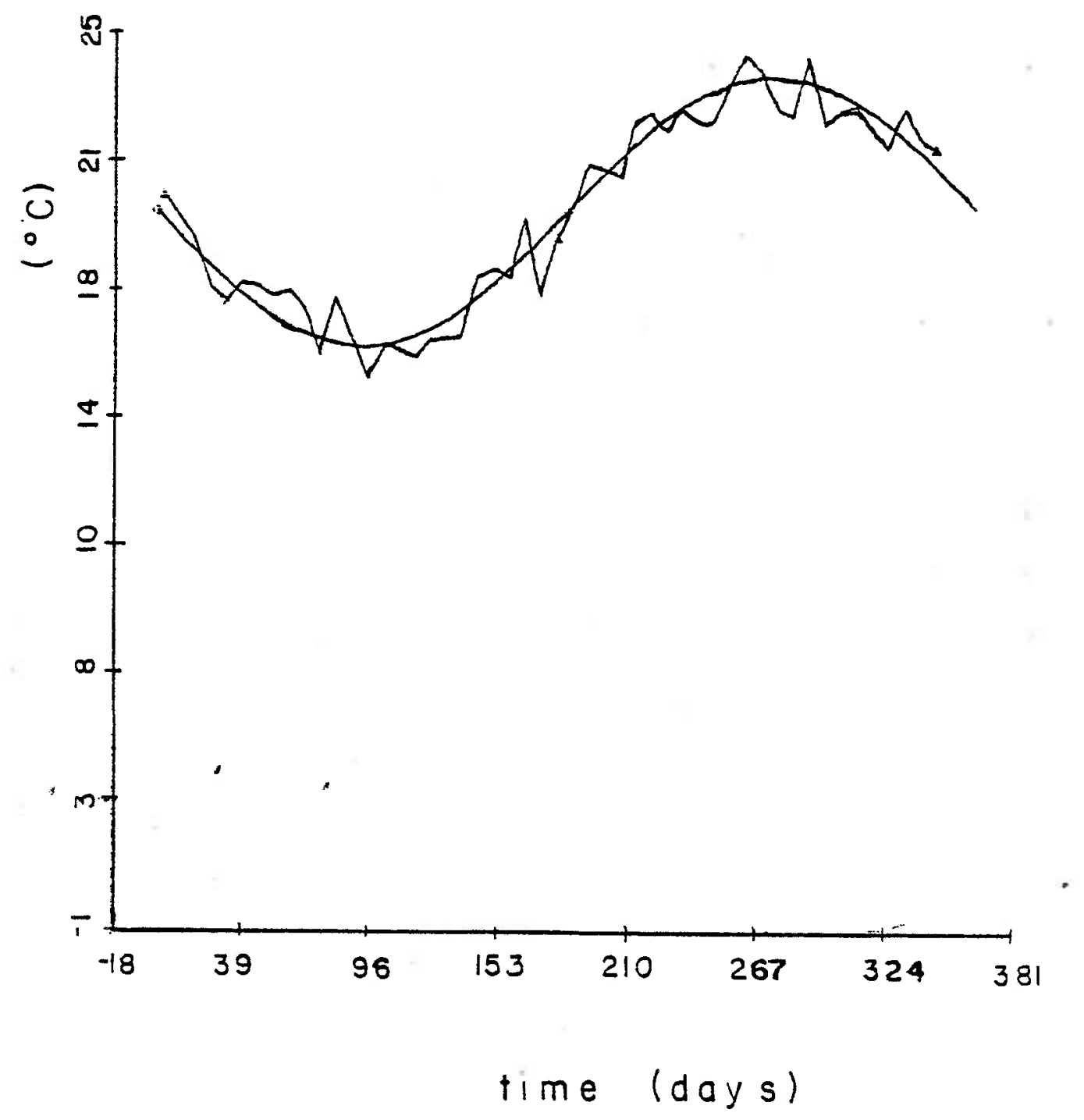

Figure C.IV.1. Comparison of Raw and Smoothed Air-Temperatures

Here we compare the two air-temperature cycles used to compute the responses of Thompson's model and Eqs. IV.1. The 'raw' air-temperature cycle is actually a seven day average of the employed temperature cycle. 


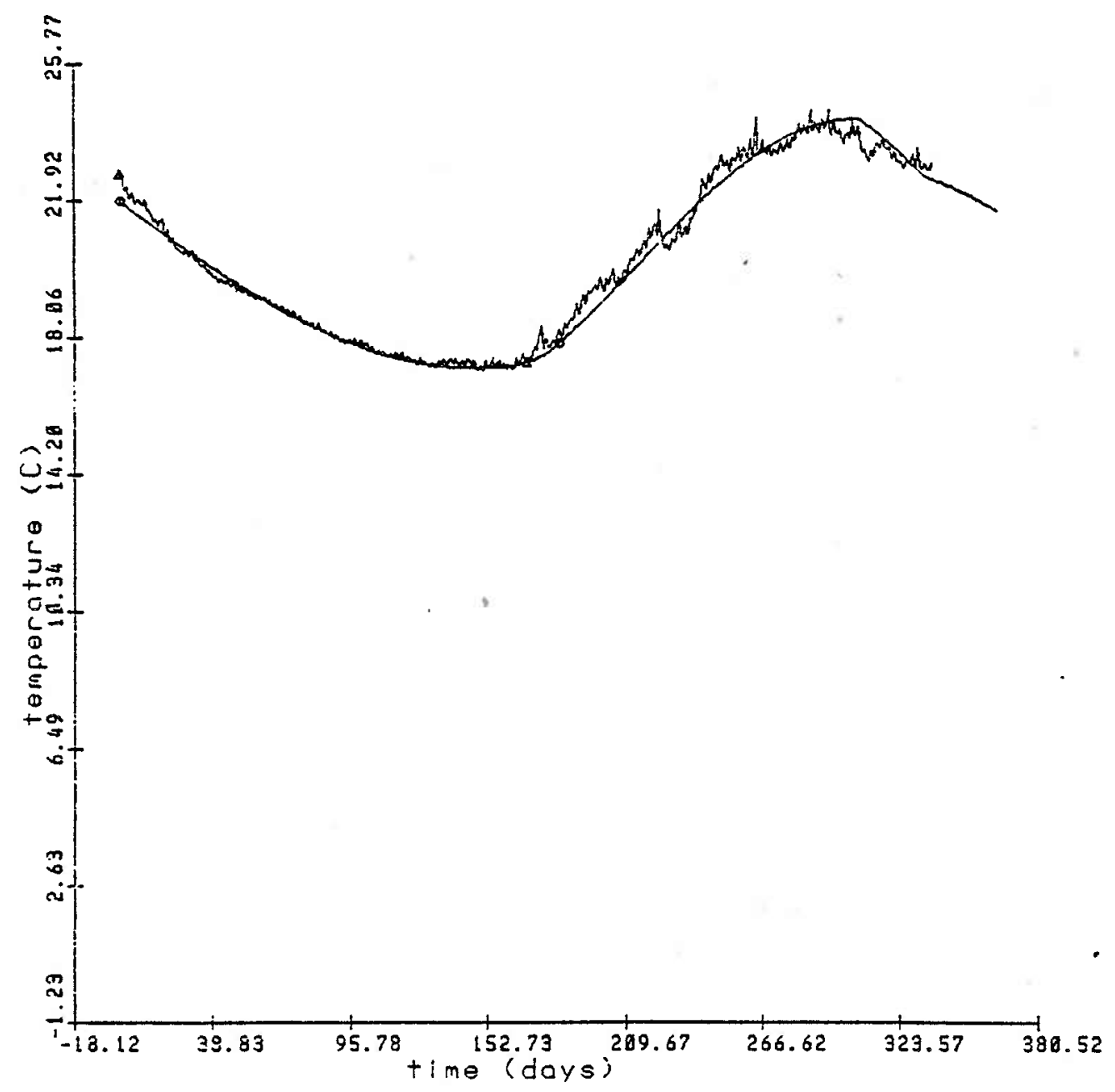

Figure C.IV.2. Comparison of SST from Thompson's Model and Eqs. IV.1

Here we compare SST as computed by Thompson's model, and by Eqs. IV.1, subject to the air-temperature cycles displayed in Fig. C.IV.1. In Eqs. IV.1, ho $=30 \mathrm{~m}$. We have neglected penetrative radiation and have run the comparison for one year. The agreement between the predicted SST's is rather striking. 


\section{CHAPTER V. THE WIND FORCED SPIN DOWN}

OF GULF STREAM RINGS

\section{V.a Introduction-}

The world's oceans on a planetary scale constitute a dispersive medium; so an isolated pressure pattern governed by linear dynamics will rapidly disperse. If the non-linear evolution of the same pressure pattern is computed, the rate of dispersive spread will be markedly decreased. For example, the decay of a Ring caused by linear wave radiation occurs in $O(6$ months) (Flierl, 1977), while wave radiation by a nonlinear Ring effects at most an energy loss of secondary importance. Perhaps the most convincing demonstrations of this were the numerical Ring simulations of McWilliams and Flierl (1979) and Mied and Lindemann (1979). In their experiments, it was found that the dynamic tendency for Ring persistence was so strong that the decay which was occurring was mostly due to non-conservative processes, e.g. friction. In McWlliams and Flierl's equivalent barotropic simulations, most $(79 \%)$ of the subsidence of the thermocline could be accounted for by a non-dispersive, frictional Ring model. In their two layer numerical simulations, dispersive Ring decay was increased by the presence of the barotropic mode, but friction still accounted for a sizeable fraction of the total Ring energy loss. Even a reduction of the viscous coefficient by a factor of 10 was insufficient to remove the overall dependence of Ring decay on friction. In agreement, Mied and Lindemann noted that different viscous coefficients produced markedly 
different time series of Ring amplitude. From these results, we would conclude that frictional processes play a major role in the decay of oceanic Rings.

This is both interesting and somewhat unsettling. First, in the numerical experiments, the viscous coefficients were purposely assigned very small values in order to minimize the influence of non-conservative processes, which, therefore, seemed to be having a disproportionately large effect with respect to Ring amplitude. Second, the dependence of amplitude decay on viscosity is at best bothersome given that viscosity is generally rather crudely modeled, and that little is currently understood about oceanic friction. In the present chapter, we are going to compute the Ring decay induced by wind forcing, using McWilliams and Flierl's model, in an effort to replace the ad-hoc viscosity in that model as the first order means of Ring spin-down, and to decide if oceanic Rings are so affected. One advantage of doing so comes from the fact that the relevant coefficients can be computed in terms of well known quantities.

Observations of Ring Decay-

Real Rings are observed to age/decay, which is characterized by a relaxation of Ring isotherms towards their resting state depths. From direct observations, Parker (1971) found that the average vertical velocity of the $17^{\circ} \mathrm{C}$ isotherm in cold Rings was roughly $50 \mathrm{~cm} /$ day. More recent direct measurements of subsidence put this at $(60-100) \mathrm{cm} /$ day (Vastano, Schmitz, and Hagan, 1980). Cheney and Richardson (1976) 
concluded from energetic considerations that Rings survive for roughly 2 years. Notice that this lifespan agrees with that computed from Parker's subsidence rate applied to an isotherm initially 300 meters from a resting depth. Such deflections of temperature surfaces are common in Rings (see Fig. IV.11).

A fuller understanding of Ring decay would allow us to make many useful statements with regards to property exchange, and therefore the importance of Rings in the general circulation. Previous estimates indicate that Slope Water/Sargasso Sea exchange due to Rings is large. Implicit in these has been the assumption that Rings disintegrate after formation, thus leaving in the host region all of the anomalous parent region material garnered at formation (the Ring Group, 1981). Recently the general perception of Ring death has shifted from disintegration to a view that Rings are removed from the circulation through absorption by the Gulf Stream (Richardson, 1980). This being a sort of reverse of formation, that water present in the Ring trapped zone at coalescence, possibly composed of a substantial fraction of the original water mass, is returned to the parent region. The total Ring-produced exchange between the regions is then closely linked to the modification of the trapped water mass by the host region (Schmitz and Vastano, 1975). As a Ring decays, vertical circulations are set up; from an understanding of the decay, we can estimate where the flow advects into or out of the trapped zone (see Fig. V.1) and therefore infer what parts of the host region water column invade, or are invaded by, the Ring. From such information, we can begin to make educated guesses about what types of Slope Water materials enter the Sargasso and vice versa, and perhaps the quantities involved. 


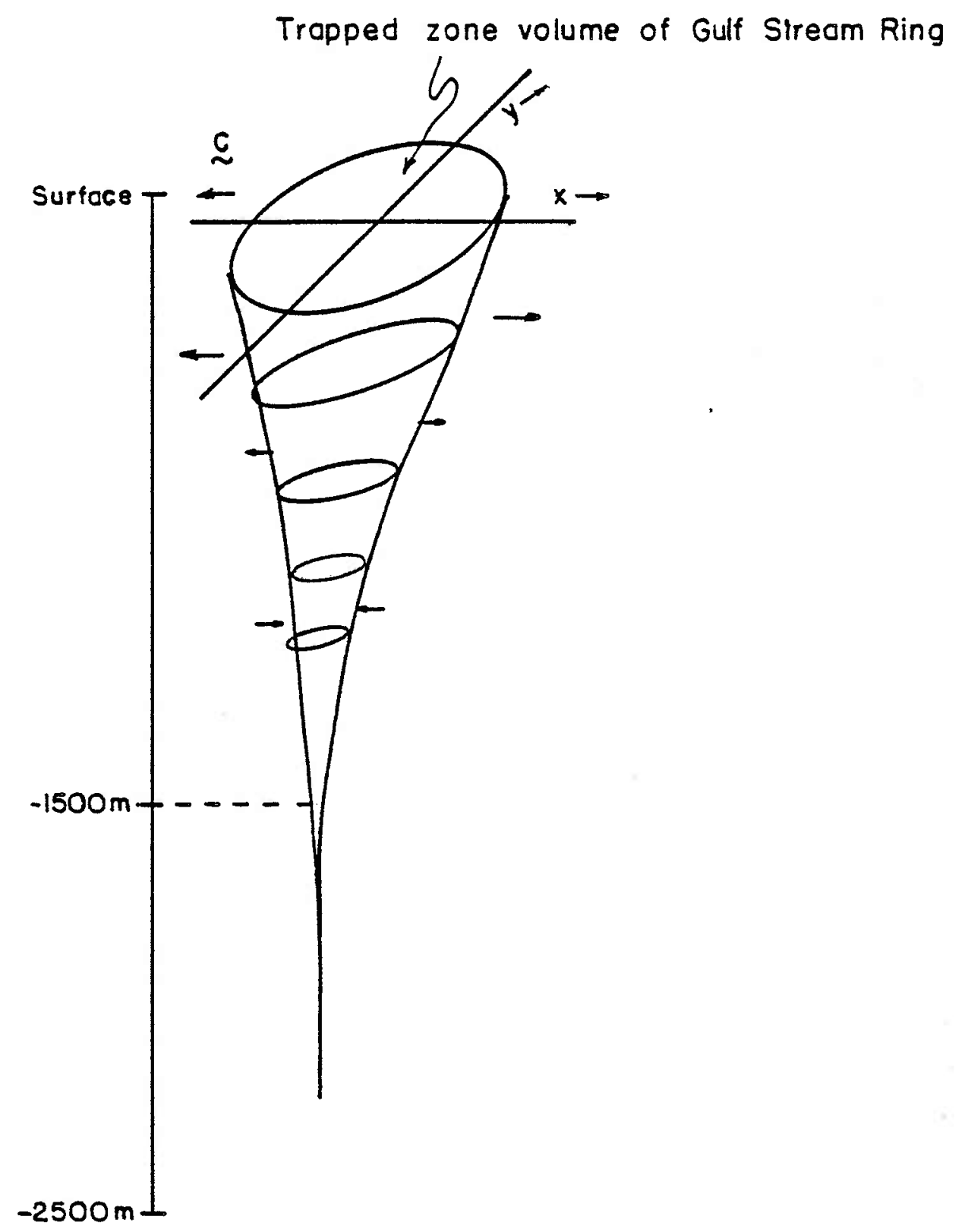

Figure V.1. Schematic of a Gulf Stream Ring Trapped Zone

Here we show the vertical distribution of the trapped zone. The arrows are indicative of inflow/outflow as set up by warm Ring decay. The depths at which mass exchange occurs influences the exchange of biology and chemicals. 
Consider how stress is affected by the presence of surface water velocities. An eastward wind, blowing at a speed of $1 \mathrm{~m} / \mathrm{sec}$ over the surface of water also moving to the east at a speed of $1 \mathrm{~m} / \mathrm{sec}$, can not transfer any momentum to the water. Conversely, the stress by the same wind on water flowing to the west at $1 \mathrm{~m} / \mathrm{sec}$ is greater than that on water which is motionless. Both statements derive from the fact that stress is a nonlinear function of the relative speed of the air and water. Consider now the consequences of an eastward wind blowing across a warm core Ring (see Fig. V.2). At the northern edge of the Ring, the water is being accelerated by the stress and hence is capable of flowing up the pressure gradient, or into the Ring. To the south, the fluid is decelerated and in response flows down the gradient, or away from Ring center. Since the momentum flux into the ocean from the atmosphere is greater where the water is flowing against the wind, the southward mass flux in the surface boundary layer is greater to the south. Mass conservation then requires an upwelling in the center of the Ring, which can either elevate the main thermocline or generate cyclonic relative vorticity. Both decelerate the Ring.

The interaction of the wind with a sheared current has also been considered by Stern $(1965,1966)$ and Niiler (1969). In these papers, the divergence driven by variations in the Ekman transport due to a non-constant local rotation rate, $\left(f+v_{x-u y}\right)$, was computed. Nifler attempted to explain anomalous isothermal structure under the surface layer of the Gulf Stream in terms of the induced upwelling, and Stern considered the forced response of geostrophic eddies. This divergence is proportional to second order derivatives of the velocity and, as 

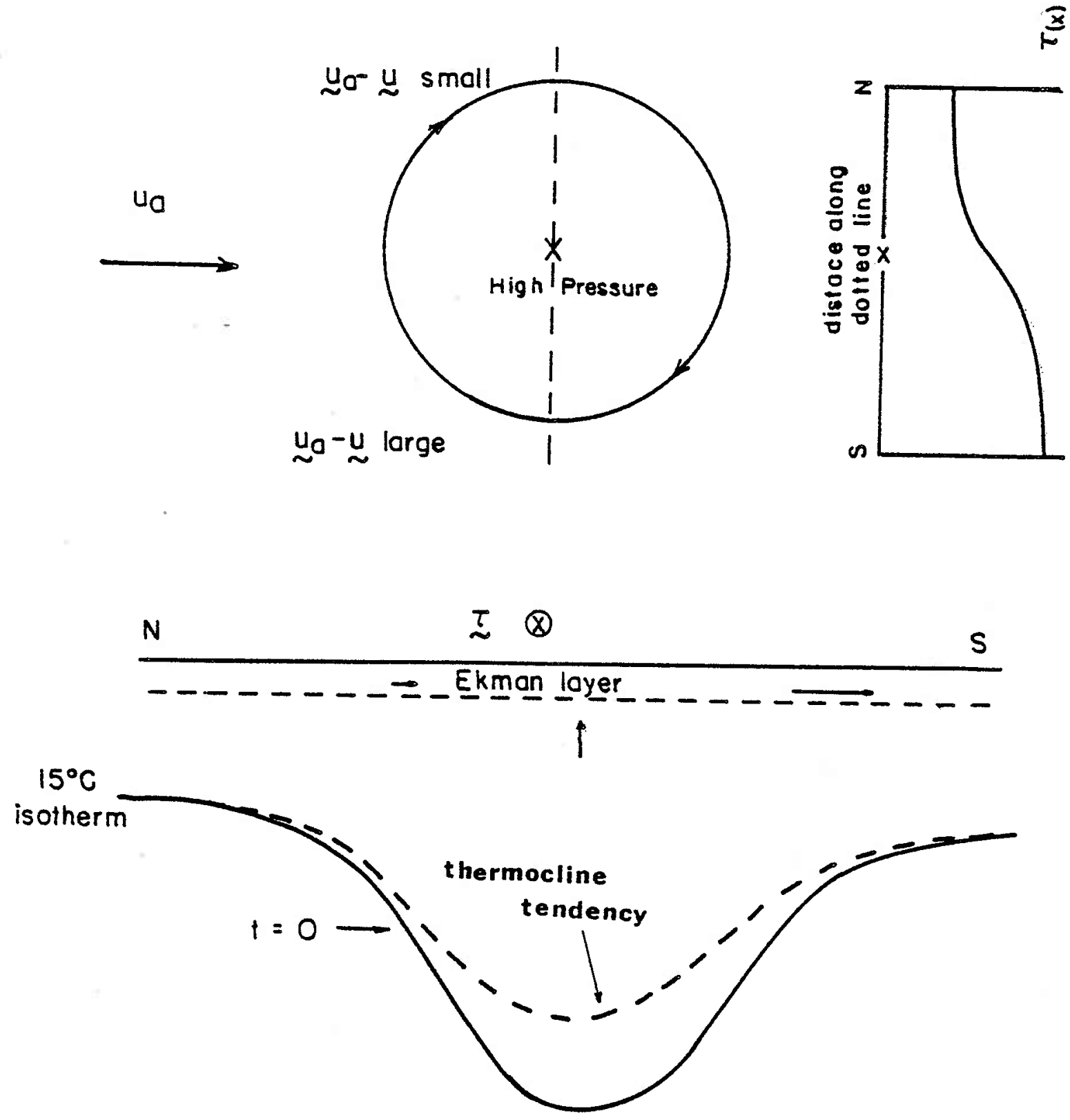

Figure V.2. Spin-down Schematic

In (a), we show the effect of an eastward wind blowing across a cyclonic Ring, a temperature transect of which is shown in (b). Because the speed of the wind relative to the water is greater to the south, the stress is greatest there. In (b) is a schematic diagram of the effect of the pumping on the interior. The solid line is the thermocline, and the dashed line, the tendency of the thermocline due to $\mathrm{w}_{\mathrm{e}}$. 
Stern showed, forces a translation of an eddy. Therefore, since the eddy is moved rather than dissipated, and this divergence operates on smaller scales than that due to variations of the relative air-sea velocity (Stern's $w$ is proportional to $u_{x x}$, and the present $w$ to $u_{x}$ ), the dissipation rates computed in the present chapter will not be affected by Stern's and Niller's mechanism. We shall, however, consider it further in Chapter VI.

\section{V.b Ekman Pumping-}

The formula for wind stress is:

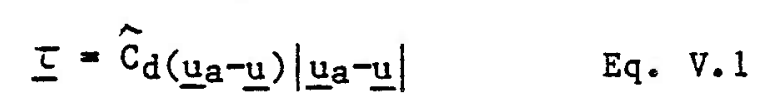

where $\hat{c}_{d}$ is an empirically determined drag coefficient, ua the wind velocity, and $\underline{u}$ the water velocity (see Table V.1). In terms of Fig. V.2, a constant eastward wind blowing across the surface of a Ring:

$$
\underline{I}=\hat{C}_{d(u a-u, u)\left((u a-u)^{2+v 2}\right)^{1 / 2} . \quad \text { Eq. V.2 }}
$$

Assuming $u_{a} \gg(u, v)$, we can expand the square root to obtain:

$$
\begin{aligned}
I & =\hat{C}_{d_{u a}} 2(1-2 u / u a,-v / v a)= \\
& =\underline{\tau}_{0}-2 \hat{C}_{\text {duauji }}-\hat{C}_{\text {duav } j} \quad \text { Eq. V. } 3 .
\end{aligned}
$$

where $\tau_{0}$ is an 'undisturbed' $(\underline{u}=0)$ surface stress. The divergence of the wind driven surface flow is proportional to the curl of Eq. V.3:

$$
w_{e}=\left(\underline{k} \cdot\left(\operatorname{curl}\left(\underline{\tau}_{0}\right)\right)+\hat{C}_{\text {dua }}\left(2 u_{y}-v_{x}\right)\right) / f_{0} \quad \text { Eq. V.4 }
$$

In most oceanographic problems, the purely wind driven divergence, $\operatorname{curI}\left(\underline{\tau}_{0}\right)$, is assumed to overwhelm the second term. Interior evolution is then determined by an externally specified stress. In the present problem, we will assume that the curl of $\tau_{0}$ vanishes, i.e. that the 
Table V.l Symbols and Definitions-

\begin{tabular}{|c|c|c|c|}
\hline Symbol & & Definition & Value \\
\hline 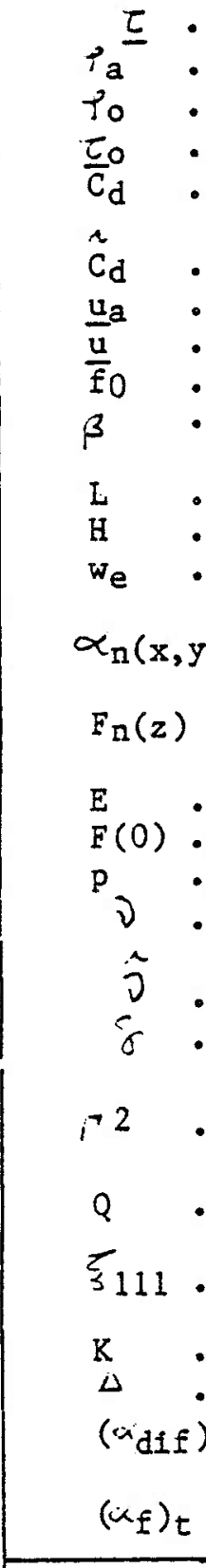 & 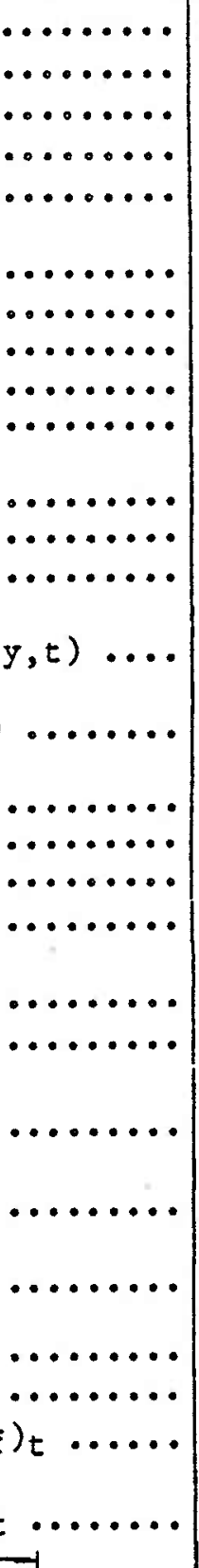 & 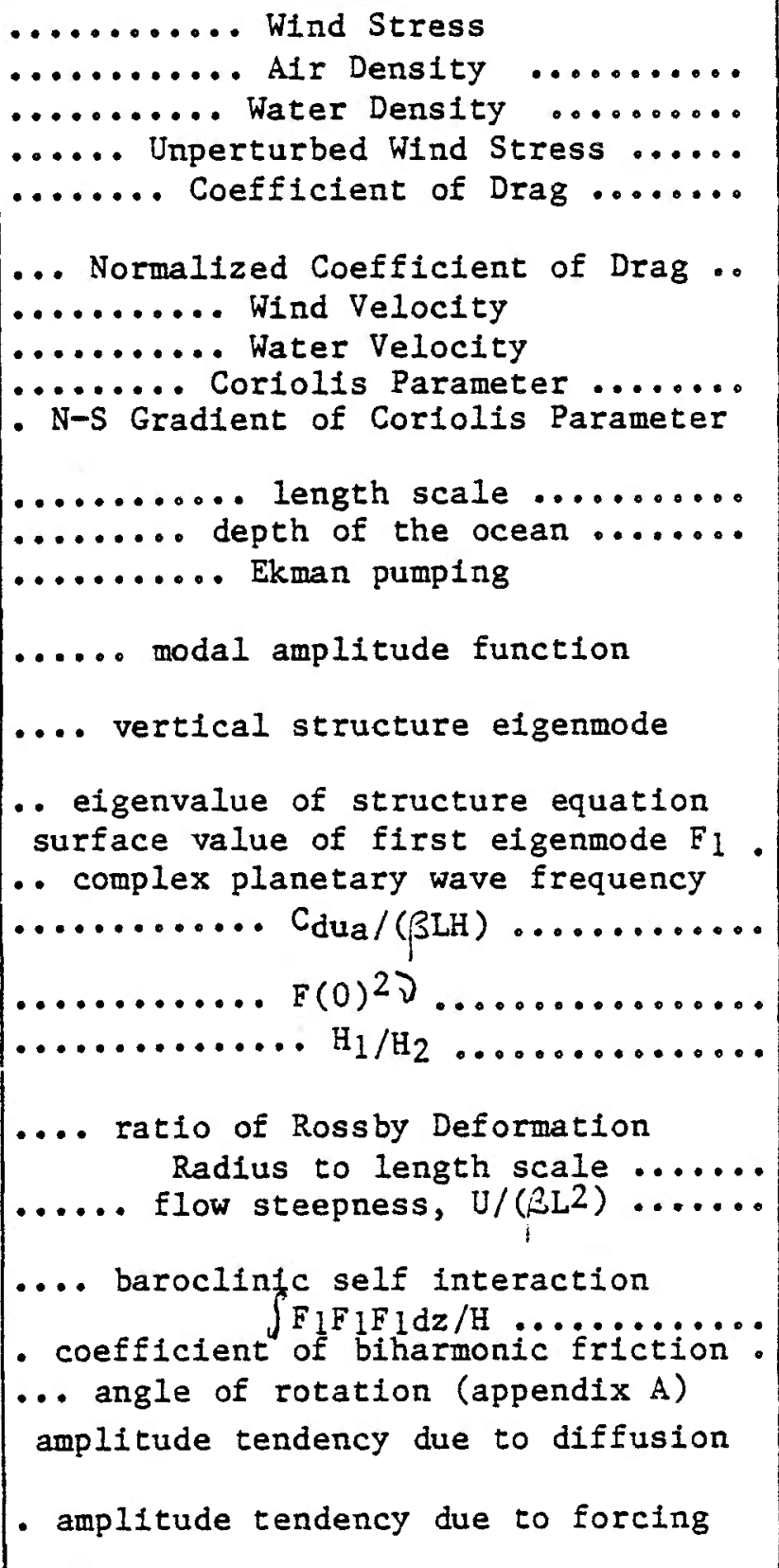 & $\begin{array}{l}10^{-3} \mathrm{gm} / \mathrm{cm}^{3} \\
1 \mathrm{gm} / \mathrm{cm}^{3} \\
\text { constant } \\
10^{-3} \\
10^{-6} \\
10^{-4} \mathrm{sec}^{-1} \\
1.7 \times 10^{-13} \\
\mathrm{~cm}^{-1} \mathrm{sec}^{-1} \\
60 \mathrm{~km} \cdot \\
5 \mathrm{~km} \cdot \\
\\
3 \\
\text { variable } \\
\text { variable } \\
\text { small } \\
2 \\
5.5 \\
5 \times 10-5 \\
\\
5\end{array}$ \\
\hline \multicolumn{4}{|l|}{ Operators } \\
\hline $\begin{array}{l}\because 2 \\
\nabla 6\end{array}$ & & $\begin{array}{l}\ldots \ldots \ldots \ldots \alpha^{2} / \partial x^{2}+2 \alpha 2 / d y^{2} \\
\cdots \ldots \ldots \ldots \ldots\left(v^{2}\right)^{3}\end{array}$ & \\
\hline
\end{tabular}


eastward directed wind is free of shear. Realistically, curl( $\left.\underline{\tau}_{0}\right)$ resides at the scales of the basin, and is therefore nearly invisible to a Ring. Hence:

$$
w_{e}=\hat{C}_{d u_{a}}\left(2 u_{y}-v_{x}\right) / f_{0^{\circ}} \quad \text { Eq. } v_{0} 5
$$

The situation described in Fig. V.2, of a mean wind and coherent surface velocity, is far too simple. The wind fluctuates on time scales ranging from seconds to years, and excites responses in the upper ocean of similar frequencies. We can account for variability in the computation of the mean stress, however the only significant change in Eq. V.5 is the replacement of $u_{a}$ with an rms measure of the wind, lual (see Appendix A.V). Without employing this notation, we shall continue to interpret $u_{a}$ as such.

From Eq. V.5, we can obtain an order of magnitude estimate of the forced vertical velocity.

$$
\begin{gathered}
w_{e} *=0(2 \hat{\operatorname{Cdua} \delta u} /(\text { foб } \mathrm{x}))= \\
=2 \times 10^{4} 10^{-6} 10^{3} 100 / 6 \times 10^{6} \mathrm{~cm} / \mathrm{sec}= \\
=30 \mathrm{~cm} / \text { day }
\end{gathered}
$$

where the $1 *$ ' denotes that we is a dimensional variable. $30 \mathrm{~cm} /$ day is a sizeable surface divergence within a quasi-geostrophic framework.

Wind drift velocities are typically a few centimeters per second, while Ring geostrophic velocities are several tens of centimeters per second; therefore, we can approximate the surface velocities by the geostrophic velocities. In terms of the interior streamfunction $\bar{\psi}$ :

$$
w_{e} *=-\tilde{C}_{d u_{a}}\left(2 \ddot{\psi}_{y y}+\hat{\psi}_{x x}\right) / f_{0}=\partial_{d} \tilde{y}^{2} \psi \quad E q \cdot v \cdot 6
$$


where we have denoted $\left(J^{2} / J x^{2}+2 J 2 / j y^{2}\right)$ by the symbol $\ddot{\nabla}^{2}$. Except for the factor of 2, the form of the pumping looks remarkably like that due to bottom friction. The ' 2 ' reflects that zonal winds are more effective at removing shear in zonal velocity than in meridional velocity. Non-dimensionally, Eq. V. 5 becomes:

$$
w_{e}=-\tilde{C}_{d u a} /(\beta L H)(24 y y+3 x x)=\partial\left(24 y y+i_{x x}\right) \cdot \quad E q \cdot V .7
$$

with a typical value for $D$ being:

$$
\begin{aligned}
\nu & =10^{-6} 10^{3} /\left(6 \times 10^{6} 5 \times 10^{5} 10^{-13}\right)= \\
& =0\left(10^{-2} / 3\right) \ll 1 .
\end{aligned}
$$

V.c Planetary Wave Spin Down-

From the form of wes it appears that wind forcing will act as a viscosity. In order to verify this claim, consider the effect of Eq. V.5 on a planetary wave.

We assume the interior is governed by quasi-geostrophic dynamics, Eq. II. 1, and that the buoyancy frequency, $N(z)$, is constant. Consider a solution to Eq. II. 1 of the form:

$$
\Psi=F(z) e^{i(k x+m y)-p t} \quad E q \cdot V .8
$$

where $p$ is a possibly complex frequency. Note that if $\operatorname{Real}(p)>0$, the wave will be exponentially damped. Substituting Eq. V.7 into Eq. II.I returns an equation for the vertical structure function $F(z)$ :

$$
F_{z z}-E^{2} F=0 \quad E q \cdot V .9
$$

where:

$$
E^{2}=s\left(k^{2}+m 2+i k / p\right) \quad E q \cdot V \cdot 10
$$


The boundary conditions become:

$$
\begin{gathered}
F_{z}=0 \quad \text { at } z=-1 \quad \text { and } \\
F_{z}=\operatorname{DFS}\left(k^{2}+2 m^{2}\right) / p \quad \text { at } z=0 .
\end{gathered}
$$

The solutions of Eq. V.9 which meet the bottom condition are:

$$
F(z)=A \cosh (E(z+1))
$$

where $A$ is an arbitrary constant. An application of the upper boundary condition returns the implicit equation:

$$
E \tanh (E)=S J(k 2+2 m 2) / p \cdot \quad E q \cdot V \cdot 11
$$

After expansion in powers of $P(<<1)$, we obtain the lowest order equation:

$$
E_{0 \tanh }\left(E_{0}\right)=0 \quad \text { Eq. V.12 }
$$

which has solutions:

$$
E_{0, n}=0, \text { in } \quad \text { Eq. V.13 }
$$

for all integer $n>0$, where we have denoted the infinitude of solutions for Eo with the second subscript 'n'. From Eq. V.13, we obtain the solutions:

$$
P_{0, n}=-i k /\left(k^{2}+m^{2}+(n \pi) 2 / S\right) \quad \text { Eq. V.14 }
$$


for the lowest order complex frequency. Notice that due to the weak surface pumping, the po,n are the Rossby wave frequencies, and the lowest order solutions for F from Eq. V.9 are simply the complete set of Rossby wave modes.

The presence of the non-homogeneous surface boundary condition is felt at the next order. From Eq. V.10 we have:

$$
\begin{aligned}
E_{1, n} & =s\left(k^{2}+2 m^{2}\right) /\left(\left(p_{0}, n\right)\left(E_{0}, n\right)\right) \\
n & >0,
\end{aligned}
$$

Eq. V.15

and, according to Eq. V.11:

$$
\begin{aligned}
& p_{1, n}=2\left(E_{0}, n\right)(E 1, n)\left(p_{0}, n\right) 2 /(S i k)= \\
= & (k 2+2 m 2) /(k 2+m 2+(n \leftarrow) 2 / S), \quad E q \cdot V .16
\end{aligned}
$$

again for n strictly positive.

In order to recover $p_{1,0}$, the complex frequency appropriate to the barotropic mode, we return to Eq. V.12. The fact that $\mathrm{p}_{1,0}$ is not determined by the above analysis is an indication that our expansion in powers of $J$ is breaking down. Such an expansion implicitly assumes $E_{O, O}$ is $O(I)$, which contradicts the solution:

$$
E_{0,0}=0
$$

from Eq. V.13. The proper interpretation of the above is that for the barotropic mode, Eo,o $<1$. An asymptotic analysis of Eq. V.12, in the limit of small $E$, shows $E=O(J 1 / 2)$. Note however that the lowest 
order solution for $P_{0}, 0$ from Eq. V.14 does not vanish. Thus, the expansion for $p$ appropriate to the barotropic mode will still require a non-zero $O(1)$ term, which turns out to be the barotropic Rossby wave eigenf requency:

$$
\text { po, } n=-i k /\left(k^{2}+m^{2}\right)
$$

The $0\left(i^{1 / 2}\right)$ barotropic solution is:

$$
E_{1,0}=\left(S\left(k^{2}+2 m^{2}\right) /\left(p_{0}, n\right)\right)^{1 / 2}, \quad E q \cdot V \cdot 17
$$

and the $O(D)$ correction to $p$ :

$$
p_{1, n}=\left(k^{2}+2 m^{2}\right) /\left(k^{2}+m^{2}\right) . \quad \quad E q \cdot V \cdot 18
$$

Notice that for both the baroclinic and barotropic modes, the correction to $p$ enters at $O(J)$; hence, the dimensional spin down time is given by:

$$
\begin{aligned}
t_{c} & =1 /\left(\partial_{\mathrm{p}_{1}, 0}\right)=0\left(\beta \mathrm{LH} / \grave{C}_{\mathrm{dua}}\right)= \\
& =300 / 10-6 \text { secs }=0(3000 \text { days })
\end{aligned}
$$

where we have used our previous estimate for $\widehat{J}$. Clearly, the effect of the surface pumping is to cause the wave to decay. 


\section{V.d Nonlinear Vortex Spindown-}

In this section, we discuss the effects of Eq. V.5 on nonlinear, coherent, quasi-geostrophic systems. Because of the complextty of the fully nonlinear equations, we will be forced to proceed numerically. The inclusion of nonlinearity, however, is essential for realistic Ring modeling, leading to long-lived numerical solutions as well as exact analytical solutions of permanent form (Flierl, Larichev, McWilliams, and Reznick, 1980).

Barotropic Mode Scaling-

The model interior equation we will use is the equivalent barotropic equation, Eq. II.14, which governs the baroclinic evolution of a two-layer system with a thin upper layer. With that configuration, the nonlinear baroclinic/barotropic interactions are weak and may be ignored, however the extent to which the barotropic mode affects baroclinic evolution through the boundary conditions must be investigated on a case by case basis. Presently, we are ignoring bottom friction as well as topographic effects, but have included surface pumping which invokes contributions from both modes:

$$
\begin{aligned}
& w_{e}=\partial\left(\sum_{n} F_{n} \ddot{\partial} 2 \alpha_{n}\right)= \\
& =\supset\left(\left(\alpha_{o x x}+2 \alpha_{o y y}\right) F_{o}+\left(\alpha_{1 x x}+2 \alpha_{1 y y}\right) F_{1}\right) \quad \text {.Eq. V.19 }
\end{aligned}
$$

(refer to Table V.l for definitions). Using the scale analysis which led to the equivalent barotropic equation, the barotropic amplitude $\alpha_{0}$ 
is $O(\delta)$ where $\delta=\left(\mathrm{H}_{1 / \mathrm{H}_{2}}\right)$ in a two layer system and is a function of $\mathrm{N} 2$ for continuous stratification. For shallow main thermoclines $\delta$ will be sma11. Similarly, $\alpha_{1}$ is $0(\delta 1 / 2)$ and $F_{1}(0)$ scales as $\gamma^{-1 / 2}$. The latter comes from the normalization condition:

$$
\int_{-1} F_{n F_{m d z}}=F_{\gamma_{n m}}
$$

combined with the constraint:

$$
\int_{-1}^{0} F_{n d z}=0 \text {. }
$$

Recall $F_{0}(0)=1$; therefore, to $O(\delta)$ the barotropic amplitude in Eq. V.19 is negligible, and the interior equation becomes:

$$
\left(\nabla^{2}-r^{2}\right) \alpha_{t}+Q^{\xi} 111 J\left(\alpha,\left(\nabla 2-r^{2}\right) \alpha\right)+\alpha_{x}=\hat{J} \tilde{\nabla}^{2} \alpha, \quad \text { Eq. V.20 }
$$

where $\hat{J}=F(0)^{2} C_{\text {dua }} /(B L H)$, the equivalent barotropic equation plus a small dissipation. Note $\hat{D}=F(0)^{2} . J$, the factor of $F(0) 2$ owing to the modal decomposition. To insure numerical stability, it is still necessary to include a higher order, enstrophy-consuming dissipation. Following McWilliams and Flierl, we employ:

$$
D=K_{0}^{6} \alpha
$$

with $K$ 'small', i.e. its reduction does not affect the present results.

Parameters-

There are a number of parameters in Eq. V.20 which affect its solutions. Ring evolution as affected by the parameters $K, \Gamma^{2}, Q$, and $\xi_{11}$ has already been the subject of much discussion in the literature. We are currently interested in observing the influence of $\tilde{J}$ and hence 
have adopted a standard set of values for the other parameters:

$$
Q=5.5, \quad r^{2}=2, \xi_{111}=1.8 \text {, and } \mathrm{K}=5 \times 10^{-5}
$$

which, with exception of $K$, are the same as the standard set used by McWilliams and Flierl. They set $\mathrm{K}=5 \times 10^{-4}$ and we have reduced this value by a factor of 10 for reasons which will become apparent. The above values for $\Sigma_{111}$ and $\Gamma^{2}$ were computed using the mean buoyancy profile from MODE (Flierl, 1978). Note, $\Gamma^{2}=2$ implies a Ring of scale $60 \mathrm{~km}$ and $Q=5.5$ a characteristic velocity of $33.7 \mathrm{~cm} / \mathrm{sec}$. Due to the modal decomposition, this scale corresponds to a depth of $0(700 \mathrm{~m})$. Because of the surface intensification of the first baroclinic mode, the appropriate surface velocity scale computed from $33.7 \mathrm{~cm} / \mathrm{sec}$ is roughly $100 \mathrm{~cm} / \mathrm{sec}$ which is an appropriate scale for Ring velocities. $\hat{j}$ includes the wind speed $u_{a}$ in its definition, and therefore will be subject to variations in the present numerical experiments. Finally, $\Gamma^{2}$ was altered from its standard value of 2 , to values of 1 and 4 , corresponding to length scales of $45 \mathrm{~km}$ and $90 \mathrm{~km}$ respectively.

Unforced Results-

McWilliams and Flierl and Mied and Lindemann discussed the unforced dynamic evolution of their model Gulf Stream Rings. One of the major points from those experiments concerned the asymmetry of the field. Although their initial conditions were chosen to be axisymmetric, the Rings quickly developed azimuthal dependence. The largest contributions to the azimuthal field came from the two closest pressure centers, whose relative signs were determined by the initial choice of Ring spin. Anticyclonic Rings developed high pressure centers to their west and low 
pressure centers to their east, and vice versa for cold Rings. In all experiments, both local extrema remained coherent with the Ring throughout the pertod of integration and apparently had an important effect on its path. McWilliams and Flierl argued that Ring propagation was largely set by the dispersion field.

Forced Results-

After repeating McWilliams and Flierl's experiments, partly to verify our numerical code and partly to generate data appropriate to an unforced Ring, we performed a set of forced Ring experiments. In Fig. V.3, we plot $\alpha$ at several different stages of evolution from one of them, a typical forced Ring experiment which will be referred to as expl0v. The remaining experiments will be catalogued in the next section. Note from Eq. II.13:

$$
d=-F \propto(x, y, t) / N^{2}, \quad \text { Eq. II. } 13
$$

that isopycnal displacement, ' $d$ ', is linearly proportional to $\alpha_{0}$ Thus these plots represent the deflection of a density surface from its resting depth. Since ' $d$ ' is of the opposite sign than $\alpha$, the Ring in Fig. V.3 corresponds to a depression in the thermocline.

The initial Ring pattern in explov was chosen to be a Gaussian:

$$
\alpha(t=0)=\exp (-r 2)
$$

in agreement with the initial condition used by McWilliams and Flierl. Subsequent development of $c<$ was very similar to that noted in their experiments. The paths of the central pressure maximum, $x_{c}^{(t)}$, which we shall take to define Ring translation, and the neighboring centers 


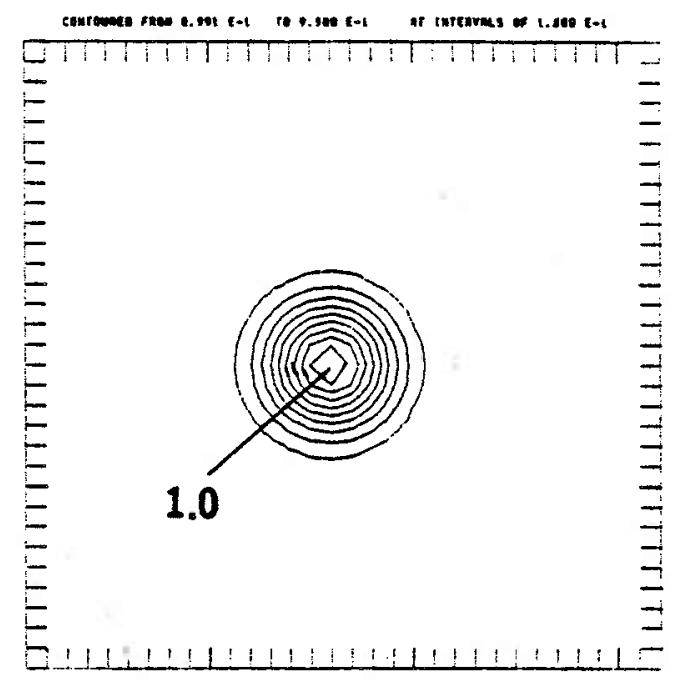

a

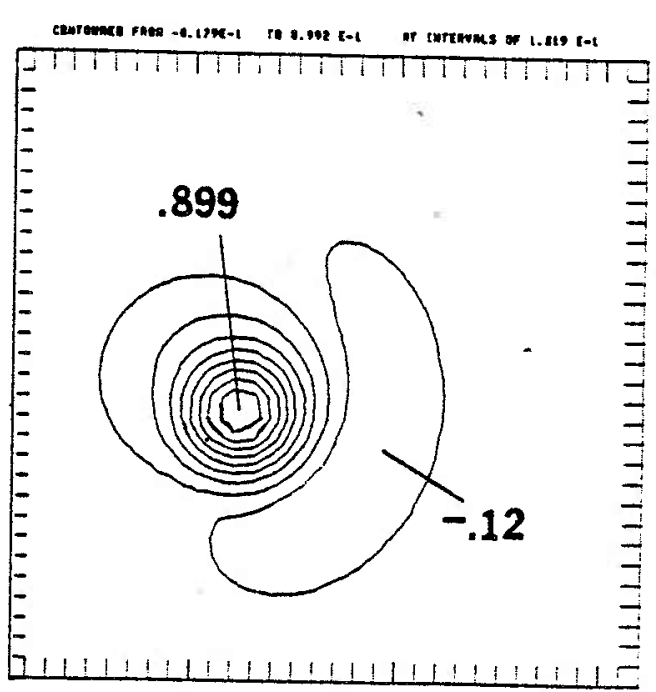

b

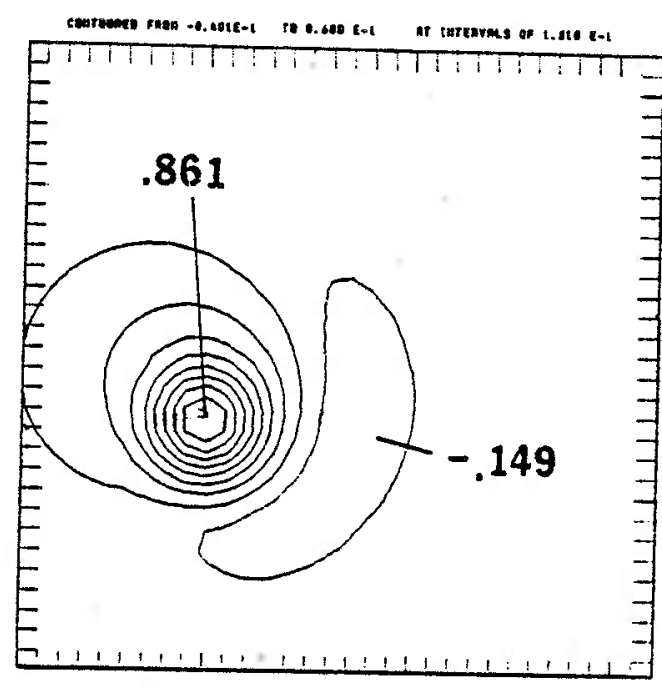

C

\section{Figure V.3. Forced Ring Evolution}

Here we have plotted the horizontal structure function, $\alpha$, as a function of time, from forced experiment explov. In this experiment, $Q=5.5, \xi_{111=1.8,} \mathrm{~K}=5 \times 10^{-5}, \quad \Gamma^{2}=2$, and $u_{\mathrm{a}}=7 \mathrm{~m} / \mathrm{sec}$. The pictures correspond to (a) the initial condition, (b) 50 days, and (c) 75 days. 
matched those of the comparable unforced Ring. This was the case in all experiments where we were able to compare analogous forced and unforced results. We can offer an explanation for this similar to one used by McWilliams and Flierl to clarify unforced Ring motion. The 'center of mass' of the system, $X$, defined by:

$$
\underline{x}=\int_{j}^{i} x \operatorname{xadA}
$$

evolves according to:

$$
\begin{aligned}
& X t=-1 / \Gamma^{2} \\
& Y_{t}=0
\end{aligned}
$$

which are found by operating on Eq. V.20. Note that the evolution of $\underline{X}$ is independent of the forcing. Deviations of Ring motion from the path of $\underline{X}$ are due to the radiation field in the vicinity of the Ring. Since the effect of $w_{e}$ is not to excite any novel structure in the developing field, but rather to cause dissipation, the location of the Ring pressure maximum is determined by wave dispersion and nonlinear interaction just as in the unforced case.

The interesting, novel behavior of the Ring caused by the wind forcing is reflected in the history of the streamfunction amplitudes. In Fig. V.4, we compare the maximum pressure amplitudes as a function of time from explov and a comparable unforced experiment (a la McWilliams and Flierl). Notice that in the wind forced experiment, there is better than a factor of two increase in amplitude decay. After 80 days, the pressure maximum of the forced experiment has lost more than $12 \%$ of its initial value, while that of the free Ring is down by $\simeq 0(5 \%)$. 


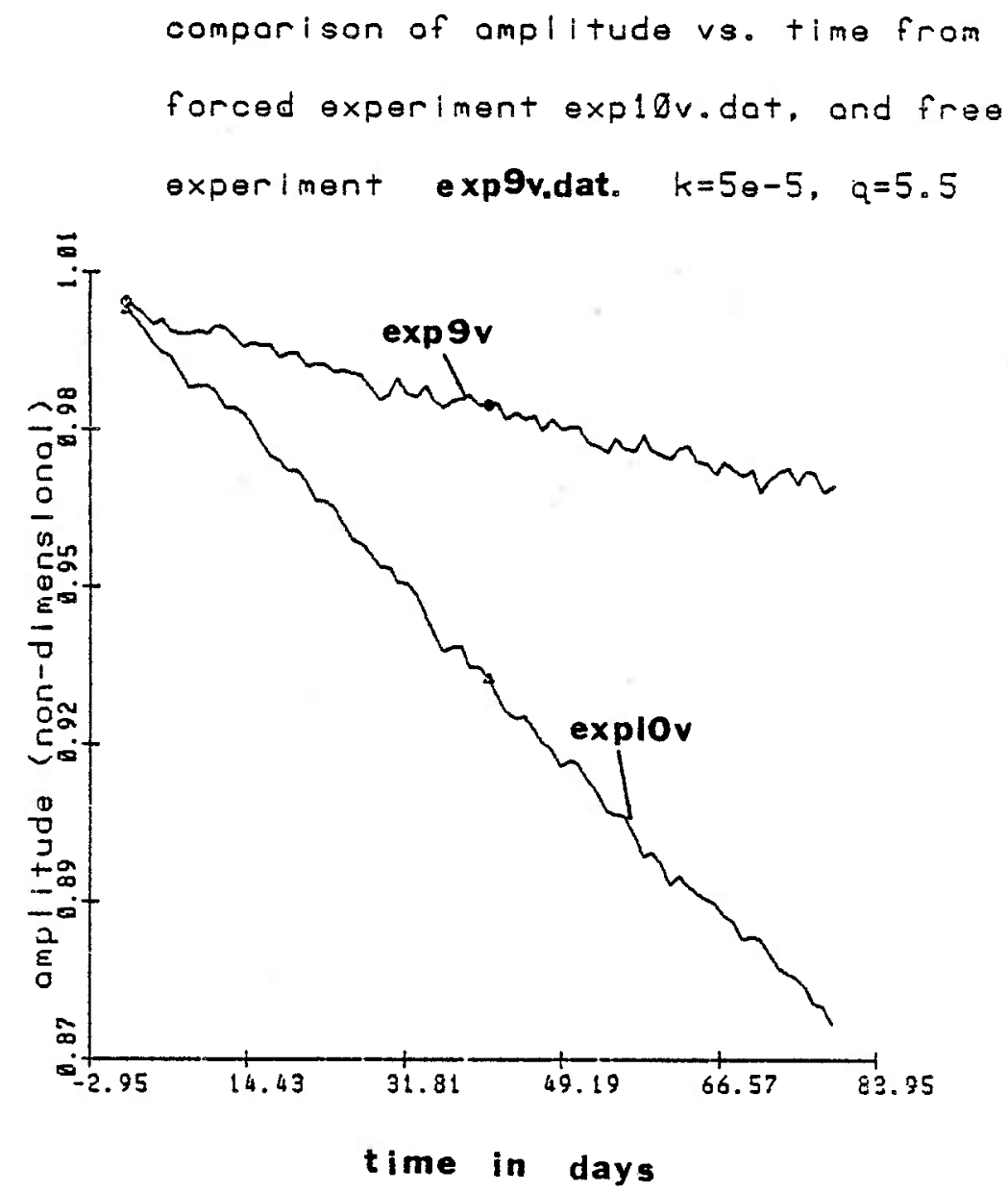

Figure V.4. A Comparison of Forced And Unforced Ring Decay

Here we compare the time histories of $\alpha_{c}$ from forced experiment expl0v and unforced experiment exp9v. The parameters are standard, with $u_{a}=7 \mathrm{~m} / \mathrm{sec}$ in the forced calculation. Note that the $\alpha_{c}$ in $\exp 10 \mathrm{v}$ decays roughly 2.5 times faster than that in exp $9 v$. 
Fig $\quad$. 5

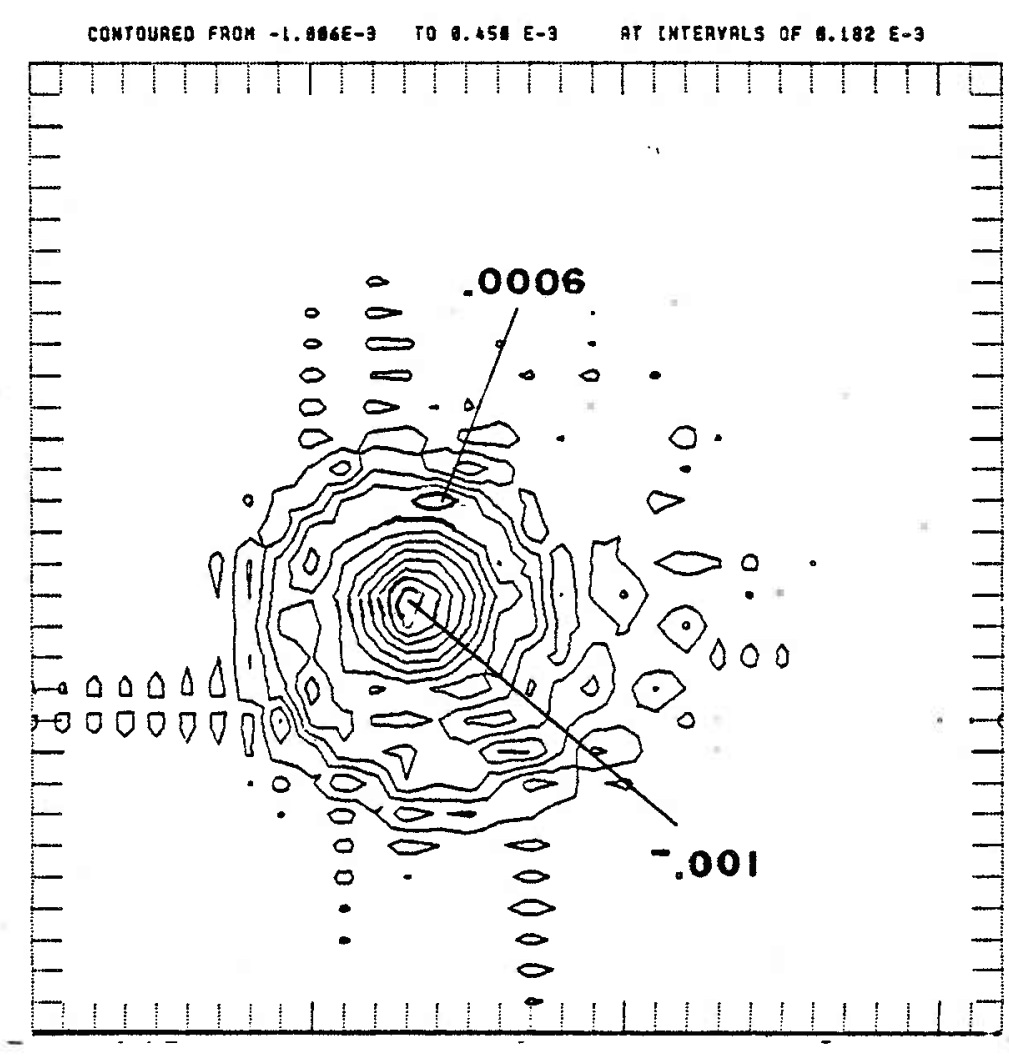

a $\left(x_{\text {dif }}\right)_{t}$

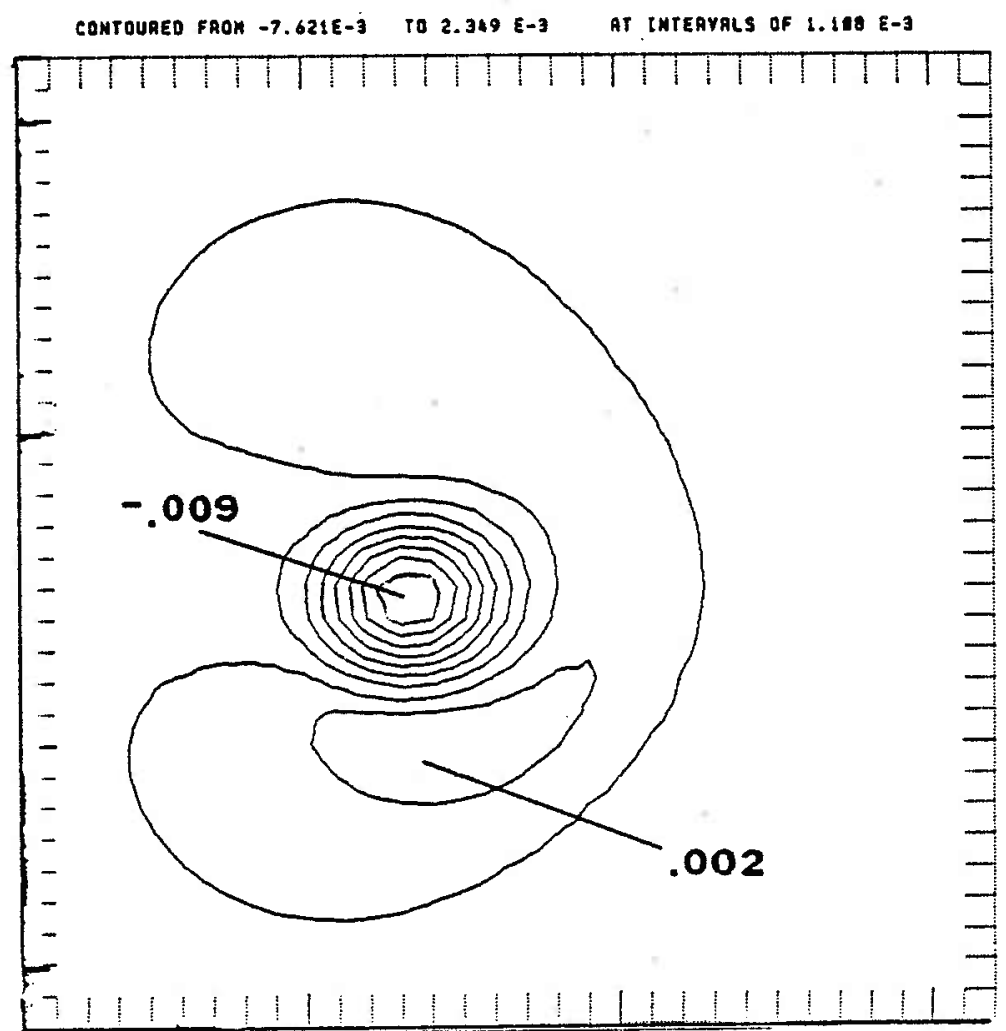

b $\left(\alpha_{f}\right)_{t}$ 
amplitude tendency due ta oll terns. from oxplovedat

TIme In doy $=40$

CONTOURED FROH $-7.688 E-1$ TO $8.399 \mathrm{E}-1$ AT INTERVALS OF $1.787 \mathrm{E}-1$

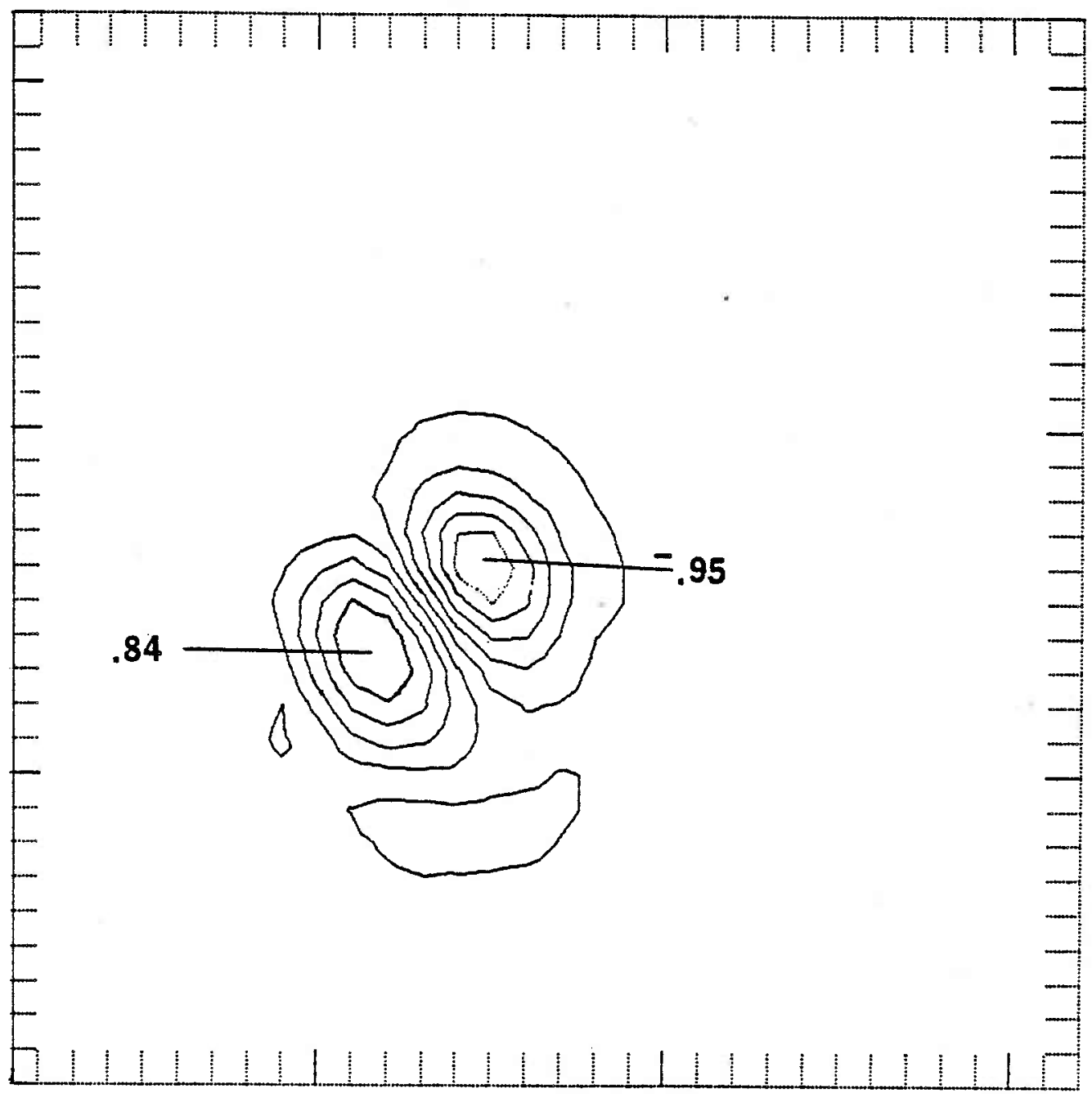

Figure V.5. Maps of $\left(\alpha_{\text {dif }}\right)_{t}$ and $\left(\alpha_{f}\right)_{t}$

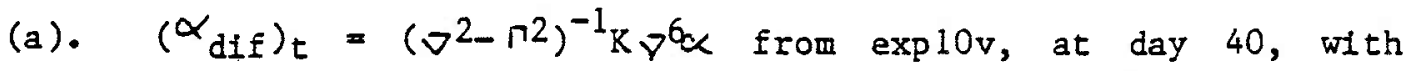
$\mathrm{K}=5 \times 10^{-5}$ and $\Gamma^{2}=2$.

(b). $\left(\alpha_{f}\right)_{t}=\left(\nabla^{2}-\Gamma^{2}\right)^{-1} w_{e}$ from exp10v, at day 40, with $u_{a}=7 \mathrm{~m} / \mathrm{sec}$.

Note that, in the Ring, $\left(\alpha_{\text {dif }}\right)_{t} \ll\left(\alpha_{f}\right)_{t}$.

(c). $\left(\alpha_{a}\right)_{t}$ from expl0v at day 40. 


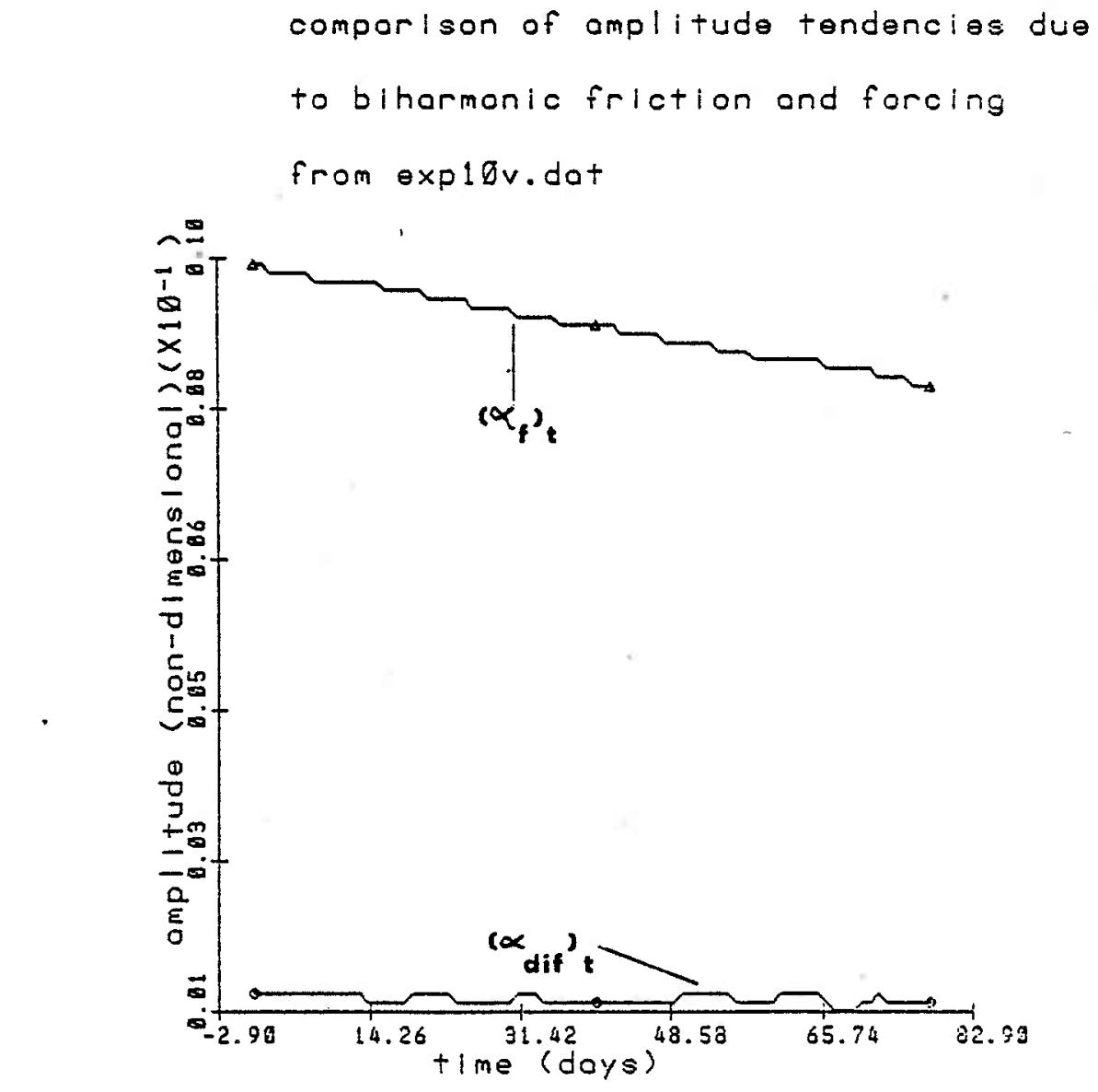

Figure V.6. $\left(\alpha_{f}\right)_{t}$ Versus $\left(\alpha_{\text {dif }}\right)_{t}$

Here we compare the magnitude of the non-conservative forces at Ring center. Plotted are $\left(\alpha_{\text {dif }}\right)_{t}$ and $\left(\alpha_{f}\right)_{t}$ for 80 days from expl0v. Note that $\left(\alpha_{f}\right)_{t} \gg\left(\alpha_{\text {dif }}\right)_{t}$. 
The Relative Importance of Forcing-

From Fig. V.4 it is obvious that the maximum amplitude decay has significantly increased in the forced experiment, and soon we shall quantify subsidence rates for comparison with those observed in the open ocean. However, in light of previous studies, it is necessary to show that the decay we are seeing is primarily the sequent of the forcing, and not of the biharmonic friction.

In order to address the question of the relative importance of friction, we numerically evaluated the decay in amplitude due to friction, defined by:

$$
\left(\alpha_{\text {dif }}\right)_{t}=\left(\nabla^{2}-\Gamma^{2}\right)-1 \mathrm{k} \nabla 6 \alpha ; \quad \text { Eq. V.21 }
$$

(see Fig. V.5.a). Similarly, we computed the amplitude tendency due to Ekman pumping, given by:

$$
\left(\alpha_{f}\right)_{t}=\left(\nabla^{2}-\nabla^{2}\right)^{-1_{w e}} \quad E q \cdot V_{0} 22
$$

(see Fig. V.5.b). Note that for the value of the mean wind speed in this experiment, $7 \mathrm{~m} / \mathrm{sec}$, the magnitude of $\left(\alpha_{f}\right) t$ in the vicinity of Ring center is generally 6 to 8 times greater than $\left(\varkappa_{\text {dif }}\right)_{t}\left(-.009\right.$ for $\left(\alpha_{f}\right)_{t}$ as compared to -.001 for $\left(\alpha_{\text {dif }}\right) t$ ); a relation which at Ring center held throughout expl0v, as Fig. V.6 demonstrates. Away from the maximum in pressure, $\left(\alpha_{f}\right)_{t}$ and $\left(\alpha_{d f f}\right)_{t}$ are somewhat more comparable, although $\left(\alpha_{f}\right) t$ tends to be larger. However, as we shall see, away from Ring center, the influence of both forcing and dissipation is relatively negligible. Consequently, we conclude that for this value of the biharmonic coefficient, $K=5 \mathrm{x} 10-5$, frictionally induced amplitude decay is of secondary importance, and that the dominant non-conservative process is 


$$
\text { Page -168- }
$$

the forcing. It was largely for this reason that we chose the above value of $K$ as standard, rather than $5 \times 10^{-4}$ as did McWilliams and Flierl. Still, the dominance of forcing as a non-conservative influence is not overly sensitive to $\mathrm{K}$; in similar experiments with $\mathrm{K}=5 \times 10^{-4}$, $\left(\alpha_{\text {dif }}\right)_{t}$ was $0(40 \%)$ of $\left(\alpha_{f}\right) t$

Finally, we compare $\left(\alpha_{f}\right)_{t}$ to the change in $\alpha$ caused by all processes, which includes advection and dispersion as well as friction. The amplitude trend due to all effects is defined by:

$$
\left(\alpha_{a}\right)_{t}=\left(v-r^{2}\right)-1\left(-J\left(\alpha,\left(\nabla^{2}-r^{2}\right) \alpha\right)-\alpha_{x}+K J^{6} \alpha+\hat{j} \tilde{\bar{v}} 2_{\alpha}\right) \quad \text { Eq. V.23 }
$$

and a map of it is included in FIg. V.5.C. First we should notice that for the most part, $\left(\alpha_{a}\right) t$ is much greater in magnitude than either $\left(\alpha_{f}\right)_{t}$ or ( $\left.\varkappa_{d i f}\right) t$. Clearly, away from Ring center, the non-conservative terms of Eq. V.23 are of negligible importance. Were we to further dissect Eq. V.23, we would find that the largest contributions to that equation are due to advection and dispersion, between which there is even a fair amount of internal cancellation. Near Ring center however, $\left(\alpha_{a}\right)$ t goes through a zero crossing. Therefore, at Ring center, a sizeable fraction of the total amplitude change can be attributed to non-conservative effects, or in this case forcing. By checking the magnitude of $\left(c_{f}\right) t$ against $\left(X_{a}\right)_{t}$ at $\underline{x}_{c}$, it was found that most $(0(50-60 \%))$ of the decay in amplitude at Ring center is due to the forcing. In this regard, we agree with McWilliams and Flierl who also attributed the subsidence of Ring isopycnals to non-conservative processes. 
V.e Subsidence Rates-

It is clear from Figs. V. 3 that the local pressure maximum is associated with what we identify hydrographically as 'Ring'. Therefore, it is the evolution of the amplitude at this point which we will compare to the field estimates of Ring decay. Using Eq. II.13, we can compute a time series of ' $d$ ' at Ring center, and hence the isopycnal subsidence associated with the numerical Ring. In order to facilitate comparison with the relevant Ring observations, the values of the derivative of the vertical structure function, $F(z) z$, and of the local buoyancy frequency, $\mathrm{N} 2$, were computed from the MODE data at a depth corresponding to the $17^{\circ} \mathrm{C}$ isotherm (Flierl, personal communication). A 'd' of 257 m was computed for the initial Ring thermocline in explov, which subsequently subsided at a rate of $.4 \mathrm{~m} /$ day.

Parameter Variations-

A series of experiments (expOv to explOv) were carried out to determine the sensitivity of the results to parameter changes. A variety of $\bar{j}$ and $k$ values were used, and $\Gamma^{2}$ was assigned values 1 $(\exp 7 v a), 2(\exp 0 v-\exp 6 v, \exp 8 v-\exp 10 v)$, and $4(\exp 7 v b)$. We interpreted changes in $\hat{D}$ as changes in ua, and to span the range of oceanic conditions, used $u_{a}=0,7$, and $14 \mathrm{~m} / \mathrm{sec}$. 0 was used in the unforced experiment. $\quad 7 \mathrm{~m} / \mathrm{sec}$ characterizes relatively quiet conditions and 14 $\mathrm{m} / \mathrm{sec}$ wintertime conditions. The mean wind was assumed to blow from west to east, except for exp4v, where the vector was oriented north to south. The values of $\mathrm{K}$ ranged from 0 to $5 \times 10-4$. In all experiments the 
Table V.2 Subsidence Rates

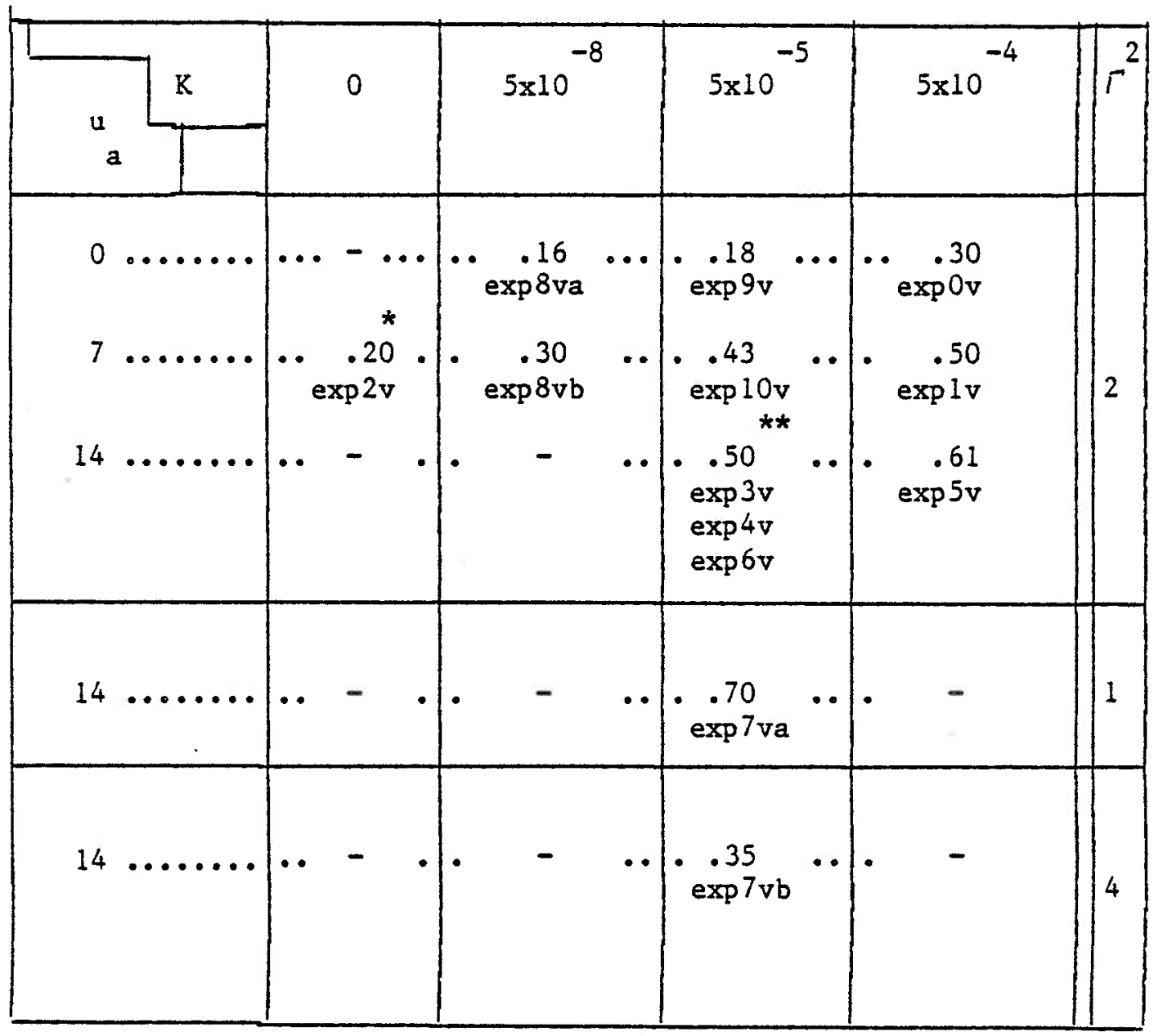

Across the top of the chart, we have listed the biharmonic coefficients, and down the side, the values of the rms wind speed ua. We group the experiments according to their values of $\Gamma^{2}$. A11 K and $r^{2}$ are nondimensional, ua are in $\mathrm{m} / \mathrm{sec}$, and subsidence rates in $\mathrm{m} / \mathrm{day}$. Note that the subsidence rates agree with observed rates of $.6 \mathrm{~m} /$ day.

\footnotetext{
In $\exp 2 \mathrm{v}, \mathrm{K}=0$ and after 60 days, the computation began to show signs of significant energy at the grid point scale. Shortly thereafter, it was judged that the calculation was becoming inaccurate and the computation was stopped. $K=5 \times 10-8$ improved the performance of the model, although the potential vorticity fields contained small scale structure. In these calculations, $\delta x=\delta y=.333$ or $20 \mathrm{~km}$.

**

Exp3v employed a time step of 14 minutes, as compared to 28 minutes for the rest. Note that the reduction made no change in the subsidence rate. In exp4v, the mean wind was blowing from the south to the north, in contrast to the west to east winds of the others.
} 
shape of the evolving pressure field was very similar to that portrayed in Fig. V.3, with the field moving to the southwest and developing a weak azimuthal asymmetry. The motions of the neighboring extrema in pressure, and the Ring, matched well between all comparable forced and free experiments. The only sizeable dependence of Ring propagation on any parameter was on $\Gamma^{2}$, where it was noticed that smaller (larger) $\Gamma^{2}$ resulted in a faster (slower) Ring. This agrees with the center of mass calculations of the previous section. Subsidence rates from the various experiments along with the relevant parameters are listed in Table V.2. Note that the larger decay rates are assoclated with larger wind speed, as expected, and that there is a dependence of decay rate on $\Gamma^{2}$. The smaller $\digamma^{-2}$, the more quickly the Ring decays, indicating that the bigger the Ring with respect to the deformation radius, the more difficult it is for surface forcing to decelerate it. Note that in all cases, the computed vertical velocity appropriate to the $17^{\circ} \mathrm{C}$ isotherm is $10^{\prime} \mathrm{s}$ of $\mathrm{cm} /$ day, which compares favorably with Parker's observed rate of 60 $\mathrm{cm} /$ day. The agreement is encouraging; it would appear that forced vortex spindown is an important component of the evolution of a Gulf Stream Ring.

\section{V.f The Spin Down Mechanism-}

Because of the agreement between observed and model spindown rates, it is worthwhile to investigate the specified technique by which the Ring is decelerated. For this purpose, we shall investigate exp $6 \mathrm{v}$, which employed $a \mathrm{~K}$ of $5 \times 10^{-5}$ and an $\mathrm{rms}$ wind speed of $14 \mathrm{~m} / \mathrm{sec}$. Comparisons of $\left(\alpha_{\text {dif }}\right) t$ and $\left(\alpha_{f}\right)_{t}$ (see previous section) show that, in 

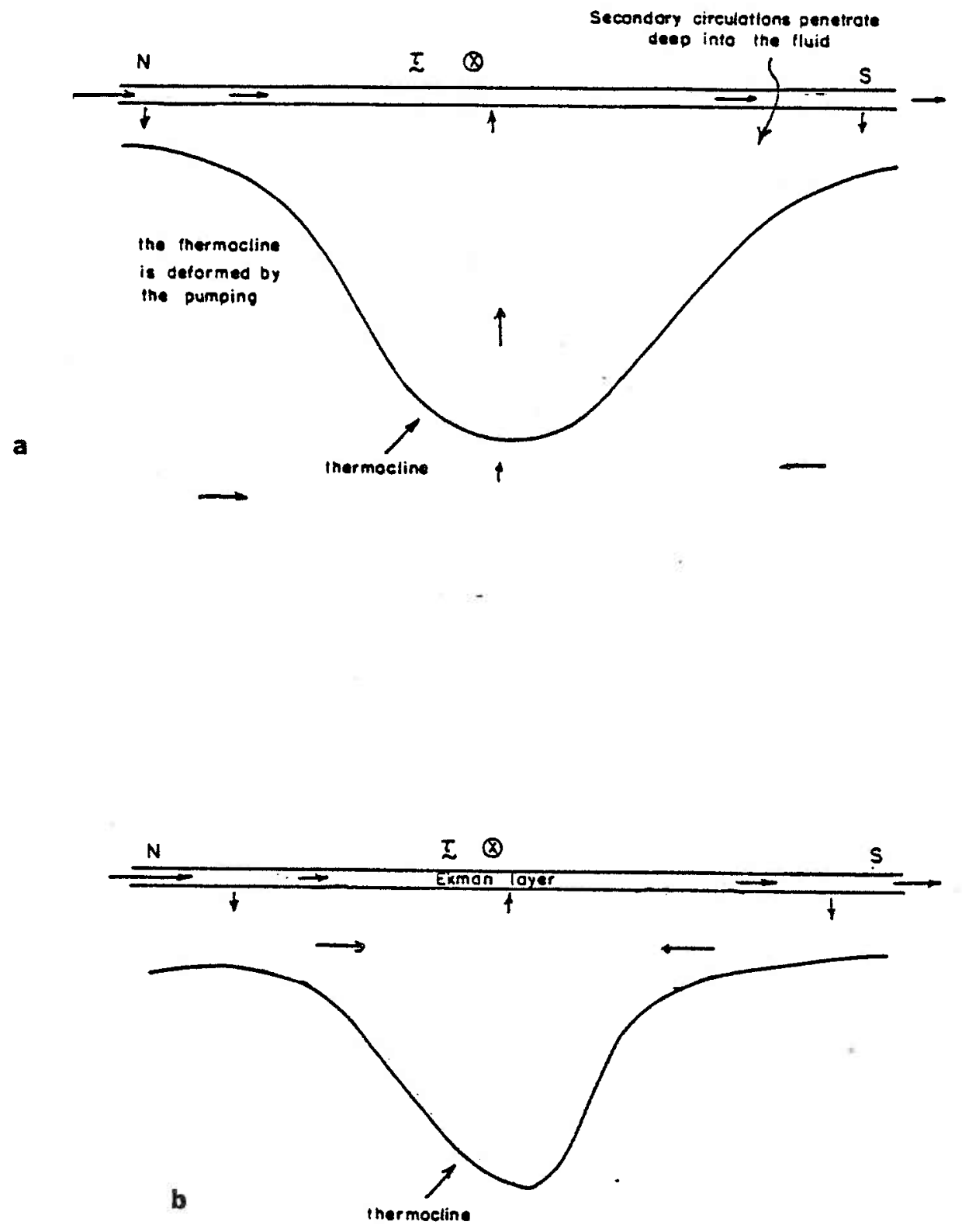

Figure V.7. Schematic of Possible Secondary Circulations

(a). The thermocline gives way to the surface pumping, and the secondary circulations to close in the deep fluid.

(b). The thermocline is rigid, which forces the circulations to close in the upper layers. 
exp6v, biharmonic viscosity rarely accounts for as much as $10 \%$ of the decay in amplitude. The results of this spindown analysis are typical of all the experiments.

A forced surface divergence affects the mass balance of the interior, resulting in the production of relative vorticity and vortex tube stretching. Consider a warm core Ring, in which case, at Ring center, the surface layers will remove mass from the deeper layers. If the fluid columns above the thermocline are able to resist the stretching imposed on them by the upwelling, the thermocline will be bodily lifted (Fig. V.7). Hence the depression of the thermocline will be smoothed out and the Ring will decelerate. If it is the thermocline which retains its rigidity, the upper columns will be stretched, resulting in the production of cyclonic vorticity which acts to nullify the extant anticyclonic Ring flow. The domination of one spin down technique over the other determines the basic structure of the weak vertical circulations within the Ring. A rigid thermocline forces the mass budget to close within the upper layers, which for a warm Ring entails a shallow, inward-directed circulation. If the thermocline is deformed, lower layer fluid is involved with the radially directed flows. Again for a warm Ring, the lifting of the thermocline creates a mass deficit in the deep layer which will be filled in from the sides.

From the numerical solutions, we can decide which means of deceleration is occuring. The production of potential vorticity ( $q$ ) due 
$-1.673 E-2$ 8.695 E-2 AT IMTERYALS OF 1.151 E-2

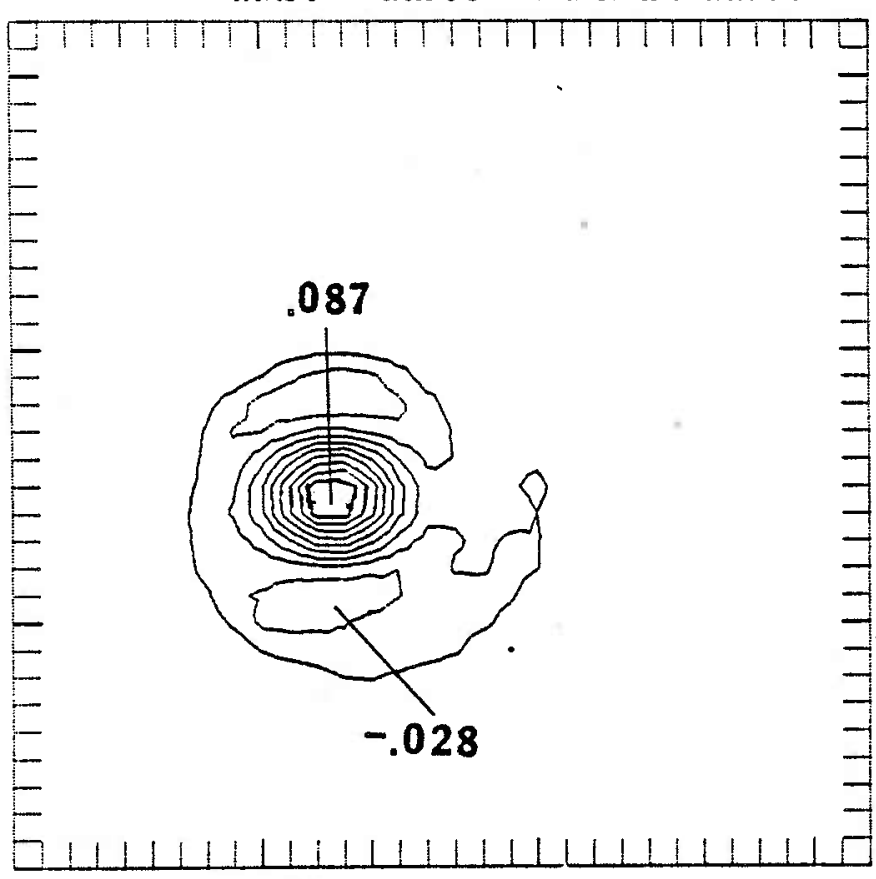

a.

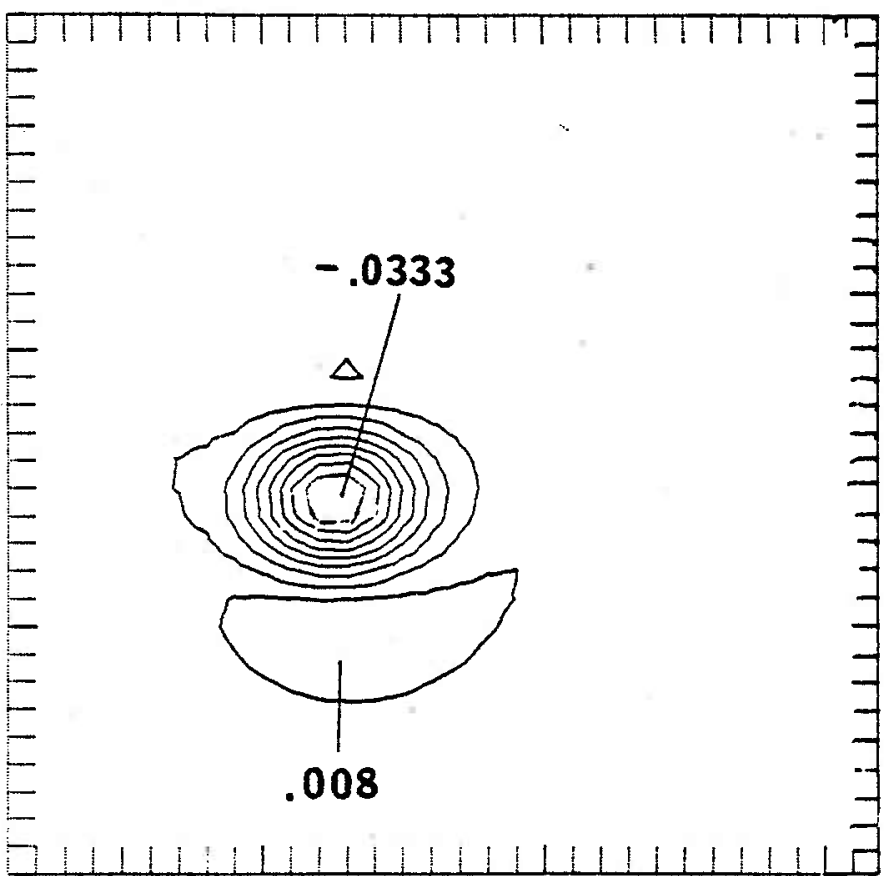

b

Figure V.8. Forced Potential Vorticity Production

(a). The generation of relative vorticity due to forcing, from exp $6 v$ at day 40 , and

(b). the generation of vortex tube stretching by the forcing, also from exp6v at day 40 .

Note that relative vorticity is generated at a rate $3-5$ times greater than stretching, indicating that for this experiment $\left(\Gamma^{2}=2\right)$, the thermocline tends to appear rigid. 
to the Ekman divergence is given by:

$$
\left(\nabla^{2}-\Gamma^{2}\right) \alpha_{t}=q_{t}=w_{e}=j \tilde{j} 2 \alpha \quad \text { Eq. V.24 }
$$

where the terms on the left hand side represent productions of relative vorticity and vortex tube stretching. The tendency of the amplitude, $r_{t}$, caused by the divergence, is given by Eq. V.22, which in combination with the above formula allows us to solve for $\nabla^{2} \alpha_{t}$ :

$$
\nabla^{2 \alpha_{t}}=w_{e}+r^{2 u_{t}} \quad \quad E q \cdot v .25
$$

Plots of both $\nabla^{2 x_{t}}$ and $\Gamma 2 \varkappa_{t}$ are provided in Fig. V.8, in which we see that for a warm core Ring, the forcing creates positive relative vorticity at Ring center and negative relative vorticity at two points located at the northern and southern Ring extremities. Recall that $u_{a}$ is from west to east. Note that the sign of the forced local rotation of the fluid is counter to that of the Ring in all three regions, and therefore acts to slow the mean flow. Similarly, the plots of $\nabla^{2 \alpha t}$ show a tendency to uplift the thermocline (or depreciate the pressure maximum). A comparison of the magnitudes in each of the plots indicates that relative vorticity production is stronger, running 3-5 times the size of vortex stretching. Similar comparisons were made in experiments with values of $\Gamma^{2}$ ranging from 1 to 4 (Ring length scales from 45 $\mathrm{km}$ to $90 \mathrm{~km}$ ), all of which suggested that the primary deceleration mechanism for Rings is relative vorticity production. Equipartition between these terms requires a larger structure, $O(200 \mathrm{~km})$, and since the present scales are typical of Rings, this spindown mechanism should 
hold for most Rings. Commensurate with this is a secondary circulation which for a warm Ring tends to be inward in the upper layers and close through the downwelling regions outside the Ring (see Fig. V.7.b).

\section{V.g Summary-}

In this chapter, we computed the wind-forced spin down of a Gulf Stream Ring in order to decide if such a mechanism is an important aspect of its evolution. The present work was motivated by experiments of McWilliams and Flierl and Mied and Lindemann, where it was shown that thermocline relaxation was strongly influenced by non-conservative phenomena. The mechanism by which the wind effects a decay derives from the nonlinear dependence of stress on the relative velocity between the air and the surface water. For a Ring, greater deceleration occurs where the water flows against the wind than acceleration where the water flows with it; hence the result is a net loss of Ring energy. It was found that the gradients of the stress could be related to the geostrophic streamfunction by a differential operator and that the concomitant divergence closely resembled that of a bottom drag or a linear frictional law. First, a sample analytical problem was completed to verify the tendency for the pumping to effect the decay of interior motions. Next, a series of numerical calculations were performed in which Rings were subjected to wind forcing and their decay was computed. Subsidence rates of isopycnal surfaces from the numerical model agreed well with field estimates, suggesting that wind forcing might well play an important role in the life history of a Gulf Stream Ring. 
A further investigation of the model results demonstrated that on these scales, the thermocline acts as though it were more rigid than deformable, and thus the mass divergence in the surface boundary was balanced in the upper layer. Consistent with this vertical circulation, the primary mechanism of vortex spin down was shown to be the production of relative vorticity. Implications of the secondary circulations with respect to the advection of oceanographic tracers were mentioned.

Wind forced decay is a physically motivated non-conservative process, with the added desirable feature that the appropriate coefficient, $\hat{J}$, does not depend on an unknown eddy diffusivity. $\hat{J}$ does depend on a parameter, $C d$, a constant characterizing air-sea momentum transfer, but it is generally felt that the value of this quantity is well-known (Francis, 1951). Given reasonable values for rms wind speed, we have demonstrated that the consequences of the induced Ekman divergence are significant and in the present calculations overwhelmed the effects of biharmonic dissipation. While still necessary to insure numerical stability in the face of an active enstrophy cascade, we were able to conclude that higher order viscosity was having a negligible effect on decay. Apparently, we have lessened the influence of biharmonic friction, for which we have no physical justification, and in its place installed a process for which we do. We have also apparenty retained the features of biharmonic viscosity which are desirable from a numerical point of view. 


\section{APPENDIX A.V WIND STRESS IN THE PRESENCE OF SURFACE FLOWS}

The high frequency variability of the wind is common knowledge. From the formula for stress, we conclude that the momentum transfer to the ocean must therefore be highly irregular. The bulk formula, Eq. V.1, requires as an input a wind speed averaged in such a way as to remove turbulent fluctuations. Still, such winds will be subject to change on intervals of days and weeks. Mesoscale flows are of a comparatively low frequency, with temporal variability on scales of several days, and are sensitive to an appropriately averaged wind stress. In the present appendix, we will derive the large scale mean wind stress, taking into account the high frequency variability of the wind and the presence of non-zero surface velocities.

We denote the average of a variable by an overbar. We shall not define the averaging process further than to say it is 'appropriate' to the mesoscale, providing structure at the Rossby Deformation scale with several day variability. Consider the mean wind stress to be a function of the averaged surface velocities:

$$
\underline{I}=C_{d\left(\underline{u_{a}-\underline{u}}\right)\left|\underline{u_{a}-\underline{u}}\right|}=\underline{S}(\underline{u}) . \quad \text { Eq. A.V.1 }
$$

We expect that the alterations of the mean stress caused by $\underline{\underline{u}}$ will be small compared to the unperturbed $(\underline{u}=0)$ stress: 


$$
\begin{aligned}
& \underline{s}(\underline{u})=\underline{s}(0)+\delta \underline{s}(\underline{u}) \quad \text { where } \\
& |\delta \underline{s}(\underline{u})| /|\underline{s}(0)|<1,
\end{aligned}
$$

in which case we can expand $\underline{S}(\underline{u})$ about $\underline{S}(0)$ to obtain an approximate form for $\delta \underline{s}(\underline{u})$.

$$
\begin{gathered}
\delta \underline{s}(\underline{u})=\left(\delta s_{x}, \delta S_{y}\right)=\quad \text { Eq. A.v.2 } \\
=\left[\frac{\partial}{\partial u} s_{x}(0) u+\frac{\partial}{\partial v} s_{x}(0) v, \frac{\partial}{\partial u} s_{y}(0) u+\frac{\partial}{\partial v} s_{y}(0) v\right]
\end{gathered}
$$

where all of the partial derivatives of $\underline{S}$ have been evaluated at $\underline{u}=0$. In terms of the above formula, the Ekman divergence becomes:

$$
\begin{array}{r}
w_{e}=\frac{1}{f_{0}}\left[\frac{\partial}{\partial x} \overline{\bar{\tau}_{(y)}}-\frac{\partial}{\partial y} \bar{\tau}(x)\right]= \\
=\frac{1}{f_{0}}\left[\frac{\partial}{\partial u} s_{y} \frac{\partial}{\partial x} u+\frac{\partial}{\partial v} s_{y} \frac{\partial}{\partial x} v-\frac{\partial}{\partial u} s_{x} \frac{\partial}{\partial y} u-\frac{\partial}{\partial v} s_{x} \frac{\partial}{\partial y} v\right] .
\end{array}
$$

where we have implicitly assumed $\operatorname{curl}(\underline{\underline{S}}(0))=0$. The derivatives of $\underline{\mathrm{S}}$ with respect to $u$ and $v$ can be evaluated using Eq. A.V.1:

$$
\begin{aligned}
& \frac{\partial}{\partial u} s_{y}=\frac{\partial}{\partial v} s_{x}=-\hat{c}_{d} \frac{\left(\overline{u_{a} v_{a}}\right)}{\left|\underline{u}_{a}\right|} \quad \text { a. } \\
& \frac{\partial}{\partial u} s_{x}=-\hat{C}_{d} \frac{\frac{\left(2 u_{a}^{2}+v_{a}^{2}\right)}{\left|u_{a}\right|}}{\mid} \quad \text { b. Eq.A.V.4 }
\end{aligned}
$$




$$
\frac{\partial}{\delta v} s_{y}=-\hat{C}_{d} \frac{\overline{\left(u_{a}^{2}+2 v_{a}^{2}\right)}}{\left|u_{a}\right|}
$$

Plugging Eqs. A.V.4 into Eq. A.V.3 returns:

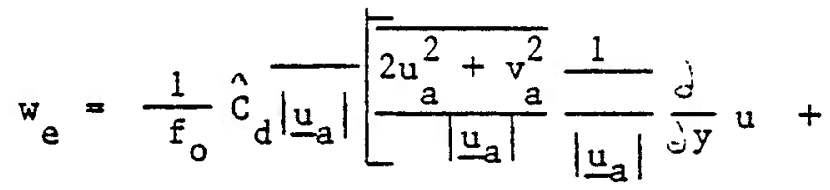

$$
\begin{aligned}
& \text { Eq. A.V. } 5 \\
& -\frac{u_{a}^{2}+2 v_{a}^{2}}{\left|u_{a}\right|} \frac{1}{\left|u_{a}\right|} \frac{\bar{v}}{\delta x} v-\frac{2 u_{a} v_{a}}{\left|u_{a}\right|} \frac{1}{\left|u_{a}\right|} \frac{j}{j x} u \\
& =\frac{1}{f_{0}} \hat{c_{d}}\left|u_{a}\right|\left(a \frac{\partial}{\partial y} u-b \frac{\partial}{d x} v-c \frac{\partial}{\partial x} u\right)
\end{aligned}
$$

which with the exception of the last term closely resembles Eq. V.5 in form. For the limiting case appropriate to Fig. V.2, i.e. for winds characterized by a mean velocity $\left(u_{a}, 0\right)$ and comparatively weak fluctuations, $\left.\left(\left(\mathrm{ua}^{\prime}\right) 2,(\mathrm{va})^{\prime}\right) 2\right)<(\mathrm{ua}) 2$, Eq. A.V.4.b is proportional to:

$$
\frac{1}{\left|\underline{u}_{a}\right|} \frac{\overline{2 u_{a}^{2}+v_{a}^{2}}}{\left|\underline{u}_{a}\right|}=\left(2+\frac{\left(0\left(\frac{\left.v_{a}^{2}\right)^{1 / 2}}{\left|u_{a}\right|}\right)\right.}{\left|\underline{u}_{a}\right|}\right.
$$


and Eq. A.V.4.C to:

$$
\frac{\overline{u_{a}^{2}+2 v_{a}^{2}}}{\left|\underline{u}_{a}\right|}=\left(1+0\left(\overline{\frac{\left(v_{a}^{\prime}\right.}{\left|u_{a}\right|}}\right)^{1 / 2}\right) \overline{\left|\underline{u}_{a}\right|}
$$

while Eq. A.V.4.a is simply:

$$
O\left(\left(\overline{\left.\left(u_{a^{\prime}}\right)^{2}\right)^{1 / 2}} \overline{\left|u_{a}\right|} \overline{\left|u_{a}\right|}\right.\right.
$$

To first order in this special case, the computed Ekman divergence is in agreement with Eq. V.5.

The coefficient in the last term of Eq. A.V.5, ' $\mathrm{C}$ ', is related to the tilt of the mean stress away from a purely zonal stress and it is always possible to orient our axes such that it will vanish. If, for example, ' $c$ ' is non-zero with respect to one frame of reference, a rotation through an angle $\Delta$ defined by:

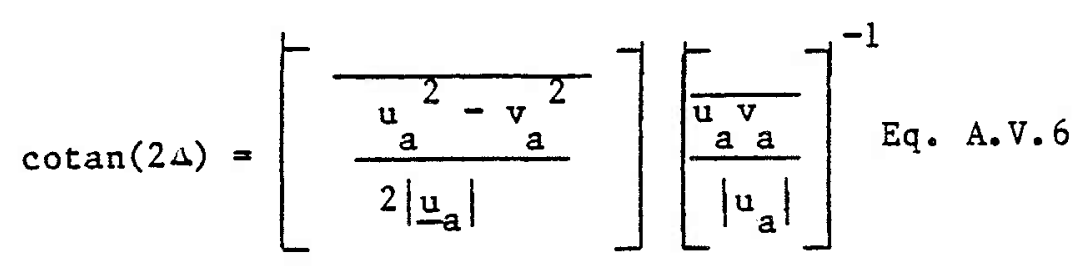

will put us in a frame such that the analogous statistic in that frame disappears. The definition of vector stress is independent of reference frame; therefore, we conclude that the final term, 'c', of Eq. A.V.5 adds no fundamentally new physics to the production of Ekman pumping. Without loss of generality, we can take the orientation of the mean stress to be purely zonal. (In fact, in exp $4 \mathrm{v}$, the mean stress was 
oriented directly to the north, with the result that the subsequent Ring. evolution was unaltered from that reported in this chapter.)

Continuing with Eq. A.V.4.b and $c$, we assume that the velocities are drawn from a parent population governed by a normal distribution function:

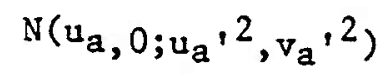

with mean vector velocity $\left(u_{a}, 0\right)$ and standard deviations $\overline{\left.\left(u_{a}\right)^{2}\right)^{1 / 2}}$ and $\overline{\left(v_{a}{ }^{\prime}\right)^{1 / 2}}$. The rms values of north-south and east-west wind speed can be computed from spectra. Under such conditions, Eq. A.V.4.b may be written as:

$$
\widehat{-c_{d} \frac{2 u_{a}^{2}+v_{a}^{2}}{\left|u_{a}\right|}=-\hat{c}_{d} \iint \frac{2 u_{a}^{2}+v_{a}^{2}}{u_{a} v_{a}}\left(u_{a}^{2}+v_{a}^{2}\right)} N\left(u_{a}, 0 ; u_{a}^{\prime 2}, v_{a}^{\prime 2}\right) d u_{a} d v_{a}
$$

$$
\text { Eq. A.V. } 7
$$

In Table A.V.1, we have listed a series of values obtained for the coefficients ' $a$ ' and ' $b$ ' of Eq. A.V.5 by integrations like those in Eq. A.V.7. Notice that the value of the coefficients do not change dramatically from the standard values $a=2$ and $b=1$ which were used in Eq. V.5. 
Table A.V.1 Values of ' $a$ ' and ' $b$ '

\begin{tabular}{|l|l|l|l|l|l|l|l|}
\hline$u_{a}$ & $v_{a}$ & $\frac{\left(u_{a}^{2}\right)^{1 / 2}}{\left(v_{a}^{\prime 2}\right)^{1 / 2}}$ & $c o h$ & $a$ & $b$ & $\left|u_{a}\right|$ \\
\hline 7. & 0. & 5. & 5. & 0 & 1.81 & 1.18 & 8.01 \\
7. & 0. & 1. & 1. & 0. & 1.98 & 1.01 & 7.03 \\
0. & 7. & 5. & 5. & 0. & 1.18 & 1.81 & 8.01 \\
7. & 0. & 10. & 10. & 0. & 1.63 & 1.36 & 10.90 \\
7. & 0. & 5. & 5. & .2 & 1.80 & 1.19 & 8.34 \\
\hline
\end{tabular}

In the above, $u_{a}$ is the mean 20 nal wind speed, $\frac{v_{a}}{\left(u_{a}\right)^{2}}$ the mean meridional wind speed, $\left(\overline{\left(u_{a}\right)^{2}}\right)^{1 / 2}$, and $\left(v_{a}\right)^{1} / 2$ the
r.m.s. fluctuations about the means, $a$ and $b$ the desired coefficients, and $\left|u_{a}\right|$ the rms wind speed as computed from the means and fluctuations. Note that we have included a category labelled 'coh', which stands for coherence. In all integrations except the last, the coherence was set to zero, which implies that meridional winds are independent from the zonal winds. In the last case, the north south-winds are slightly correlated with the east-west winds. All velocities are in $\mathrm{m} / \mathrm{sec}$; coherence, $\mathrm{a}$, and $\mathrm{b}$ are non-dimensional. 
CHAPTER VI. SOUTHWARD RING PROPAGATION AS A CONSEQUENCE OF SURFACE TEMPERATURE ANOMALIES

VI. a. Introduction

Cold air blowing across warm water is heated from below and therefore predisposed towards turbulent convection. If warm air flows over cold water, vertical motions are gravitationally opposed, and the flow is less turbulent. All else being equal, a more efficient, and hence greater, transfer of momentum from the wind to the water occurs in the turbulent flow. The empirical formula relating windstress to air velocity is:

$$
\underline{I}=P_{a C_{\text {du }}}\left|\underline{u}_{a}\right| P_{0}
$$

where $\underline{E}$ is the vector stress, $u_{a}$ the wind velocity, $P_{a}$ the density of the air, $P_{0}$ the surface water density, and $C_{d}$ the so called bulk coefficient of drag. By the above arguments, the stress is inversely proportional to the static stability of the air column; hence $C_{d}$ will increase (decrease) in magnitude for cold (warm) air over warm (cold) water. Although this reasoning is rather vague, bulk measurements of wind stress (Deardorff, 1968) corroborate the basic 1dea. In Table VI.1, we have listed measured values of $C_{d}$ as a function of air-sea temperature difference; note the $30 \%$ variations for the relatively small range of temperatures.

The effects of sea surface temperature on stress were included by Bunker (1976) in his calculation of basin scale mean wind stress. As a 
Table VI.I

The Drag Coefficient as a Function of Air-Sea Temperature Difference

(From Bunker (1976))

Drag Coefficient $\times 10^{-3}$

Air minus Sea Temperature in ${ }^{\circ} \mathrm{C}$

\begin{tabular}{|c|c|c|c|c|c|c|l|}
\hline $\begin{array}{c}\text { Wind } \\
\text { Speed } \\
(\mathrm{m} / \mathrm{sec})\end{array}$ & $>5$. & $\begin{array}{r}4.9 \\
\text { to } \\
1.0\end{array}$ & $\begin{array}{r}0.9 \\
\text { to } \\
0.2\end{array}$ & $\begin{array}{c}0.1 \\
\text { to } \\
-0.2\end{array}$ & $\begin{array}{c}-0.3 \\
\text { to } \\
-1.0\end{array}$ & $\begin{array}{c}-1.1 \\
\text { to } \\
-4.9\end{array}$ & $<-5$. \\
\hline & 0.06 & 0.60 & 0.98 & 1.20 & 1.32 & 1.56 & 1.80 \\
$.01-5$. & 0.77 & 1.30 & 1.43 & 1.54 & 1.60 & 1.78 & 1.86 \\
$5 .-10$. & 1.47 & 1.72 & 1.80 & 1.87 & 1.90 & 2.0 & 2.10 \\
$10 .-15$. & 1.95 & 2.04 & 2.10 & 2.16 & 2.22 & 2.25 & 2.32 \\
$150-20$. & 2.26 & 2.30 & 2.35 & 2.40 & 2.42 & 2.44 & 2.48 \\
$20 .-25$. & 2.52 & 2.54 & 2.57 & 2.60 & 2.62 & 2.63 & 2.64 \\
$25 .-30$. & 2.50 & & & & & & \\
\hline
\end{tabular}


result, the Sverdrup transport lines contained a thin, jet-like feature (Leetma and Bunker, 1978) near the point where the Gulf stream exits the coast, which led Reininger, Behringer, and Stommel (1979, hereafter RBS) to postulate that the narrowness of the deep ocean Gulf Stream is maintained in this way. Inherent in this idea is a subtle link between the forcing field, which drives the general circulation, and the circulation, which transports warm water. More recently, Huynh and Veronis (1981) have used a temperature sensitive coefficient of drag in a general circulation model to demonstrate its influence on poleward heat transport.

Rings, especially warm core Rings, are characterized by several ${ }^{\circ} \mathrm{C}$ contrasts in sea surface temperature (SST). Therefore, a constant wind blowing across the surface of a Ring will develop gradients due to the varying stability of the air-sea interface, and the surface flow will become divergent. This will produce a forced adjustment of the fluid, the computation of which will be the focus of the present chapter.

Consider the nature of the forced Ring problem. After Ring genesis, SST propagation and configuration are governed in large by advection. The Ring-SST system's evolution is therefore nonlinear, with a 'feedback' reminiscent of that studied by RBS. The temperature field produces an Ekman pumping which affects the interior flow; the interior flow evolves, which in turn alters the SST pattern and the concomitant vertical circulation. There is, however, a fundamental difference between the present problem and that studied by RBS. In their paper, RBS computed steady state circulations, while the present problem is an initial value problem. 
In any case, to the extent that the Ring remains a coherent structure, a certain component of its evolution will be the result of this forcing. In the next few pages, it will be suggested that the temperature dependence of the coefficient of drag results in Ring southward migration.

VI.b Scale Estimates-

The Coefficient of Drag-

In RBS, the dependence of the coefficient of drag on air-sea temperature difference was taken to be linear:

$$
C_{d}=C_{d_{0}}(1+j(T-T a)) \quad E q \cdot V I . I
$$

with $j$ being the coupling coefficient, $C_{d_{0}}$ a reference drag coeficient, $T$ sea surface temperature, and $T a$ air temperature. When necessary, we will employ Eq. VI.I with $j=.1 /{ }^{\circ} \mathrm{C}$ (RBS used .125 and $.250 /{ }^{\circ} \mathrm{C}$ ) as our model for the coefficient of drag. The functional relationship of drag and temperature in Table VI.1 is somewhat more complicated than Eq. VI.1, which is a crude model, hence, we should be wary of pressing quantitative interpretations beyond reasonable limits. St111, Eq.VI.1 captures the spirit of the drag-temperature functional dependence, and since many of the arguments presented in the text require only that stress increase (decrease) for a buoyantly unstable (stable) air-sea interface, qualitative inferences are justified.

Using $j=.1$, we notice that for $|\mathrm{T}-\mathrm{Ta}|=0\left(1^{\circ} \mathrm{C}\right)$, the entries of Table VI. 1 are reproduced well. For $5^{\circ} \mathrm{C}$ differences, the errors are more 
Table VI. 2

Scales and Parameters

\begin{tabular}{|c|c|c|}
\hline Notation & Meaning & Size \\
\hline 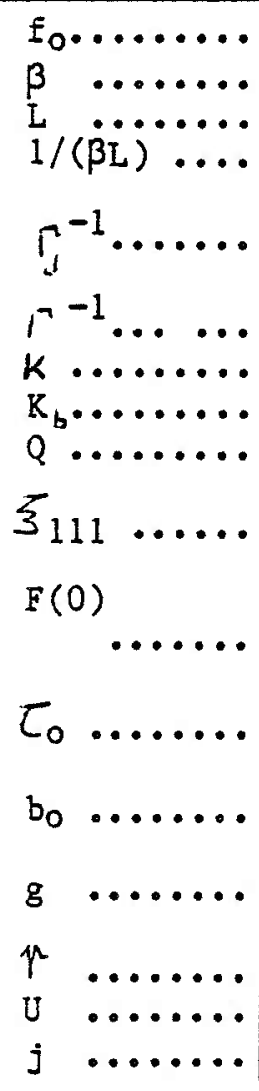 & 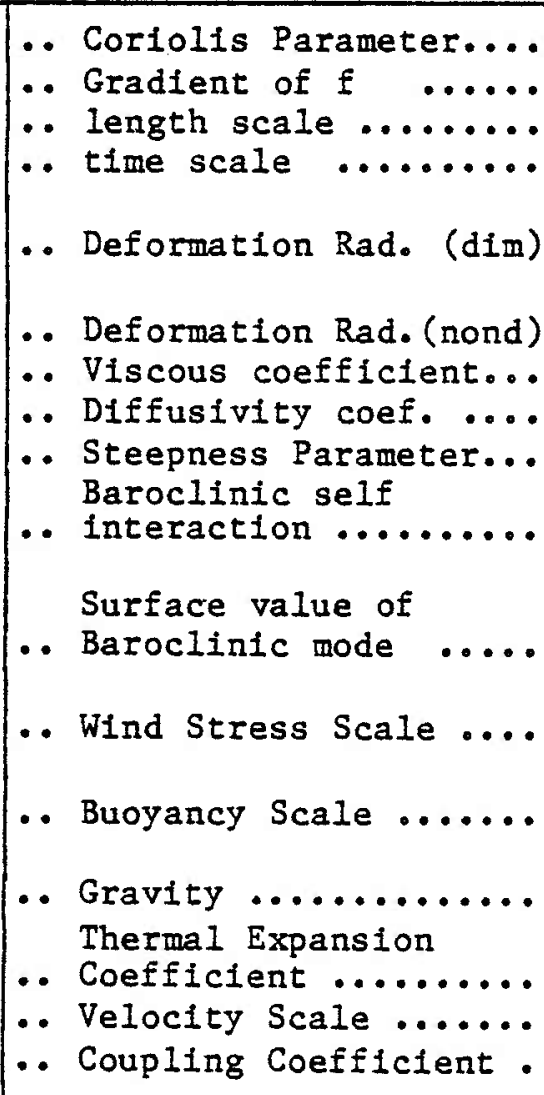 & $\begin{array}{l}1 \times 10^{-4} \\
1.7 \times 10^{-13} \\
60 \mathrm{~km} \\
11.7 \text { days } \\
42.22 \mathrm{~km} . \\
2 . \\
5 . \times 10^{-4} \\
.04 \\
4.76 \\
2.1 \\
3.0 \\
1 \mathrm{dyne} / \mathrm{cm}^{2} \\
1 \mathrm{~cm} / \mathrm{sec}^{2} \\
103 \mathrm{~cm} / \mathrm{sec}^{2} \\
2 \times 10^{-4}{ }^{\circ} \mathrm{C}^{-1} \\
29.1 \mathrm{~cm} / \mathrm{sec}^{\circ} \\
11 /{ }^{\circ} \mathrm{C}\end{array}$ \\
\hline
\end{tabular}


difficult to estimate and are strong functions of air speed, but run about $10-20 \%$.

The Ekman Divergence-

We can estimate the size of the forced vertical velocity using the information in Table VI.1. We know that:

$$
w_{e}=\underline{k} \cdot \operatorname{curl}(\underline{\tau}) /\left(f_{0}\right) \quad E q \cdot V I .2
$$

where $f_{0}$ is the Coriolis parameter, $\underline{k}$ a vertically directed unit vector, and $\subseteq$ the vector wind stress. Hence:

$$
w_{e}=1 / f_{o}(\Gamma \tau /(\delta y))=\left|\underline{u}_{a}\right|_{\underline{u}}\left(\delta \hat{C}_{d}\right) /\left(f_{o} \delta y\right) \text {. }
$$

For $\hat{C}_{\mathrm{do}}=10^{-6}, \mathrm{u}_{\mathrm{a}}=10 \mathrm{~m} / \mathrm{sec}$, a surface temperature contrast of $O\left(3^{\circ} \mathrm{C}\right)$, a length scale of $60 \mathrm{~km}$, and an $f_{0}$ of $10^{-4} / \mathrm{sec}$, we obtain:

$$
w_{e}=8 \times 10 e^{-4} \mathrm{~cm} / \mathrm{sec} \text {, }
$$

which is clearly 'big enough' to be of dynamical importance. Recall that this $w$ is the same order of magnitude as the vertical velocities in the previous chapter.

The Pumping and it's Effects-

Consider a zonal wind blowing across the surface temperature pattern of a warm core Ring, (Fig VI.1.a) and suppose the unperturbed stress based on the wind:

$$
\underline{\tau}_{0}=\hat{\mathrm{C}}_{\text {dou }}\left|\underline{u_{a}}\right| \text {, }
$$

is constant. The central region of the vortex is warm relative to the surrounding waters and is therefore a site of enhanced stress. If we were to measure stress on a transect commencing south of Ring center and 

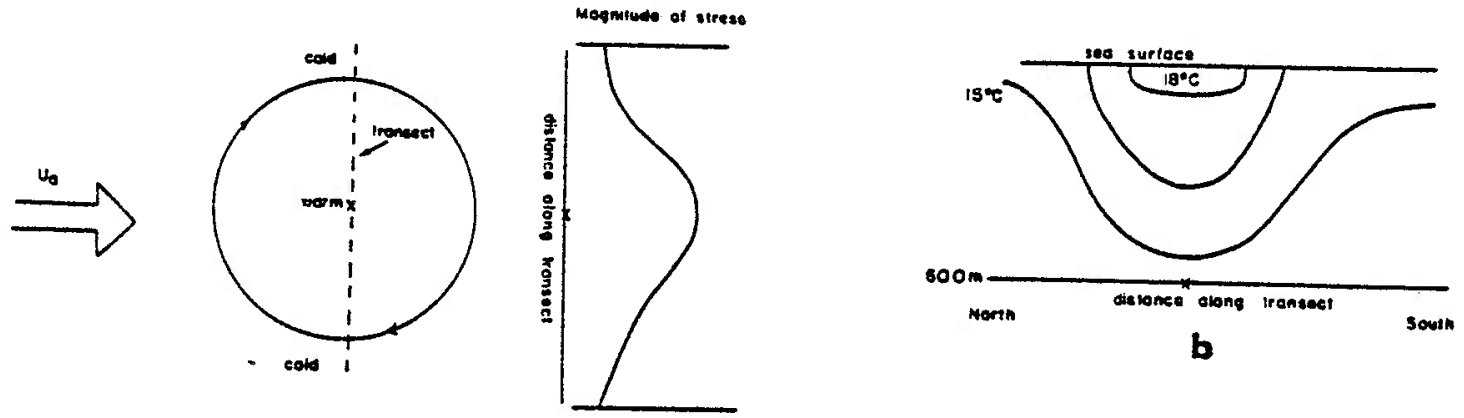

a

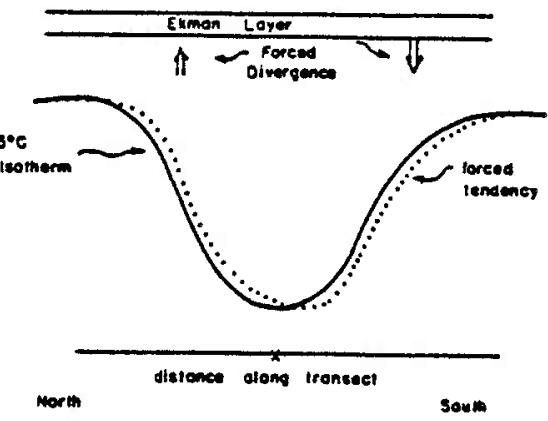

c

Figure Captions - Chapter VI.

Figure VI.1. Schematic of Ring Response to Wind Forcing

(a). An eastward directed wind crossing the top of a warm Ring stresses the center of the Ring more than the flanks due to the destabilizing effect of the warm waters.

(b). In this transect of a warm core Ring, notice the bowl-shaped isotherms with the deepest penetrations at Ring center.

(c). A schematic of the effect of the divergence produced by the stress in (a) on the isotherms in (b) is plotted. The direction of $w_{e}$ is upwards to the north, and downwards to the south, producing $a$ tendency in the thermocline for a shift to the south (the docted line). 
running due north, we would see $|\tau|$ first increase and then decrease. Therefore, the accompanying vertical divergence has both upwelling and downwelling centers, the former to the north of Ring center and the latter to the south. The temperature-depth structure of a warm Ring appears as in Fig VI.1.b. Consider a thought problem in which the thermocline is flaccid, so that the pumping simply lifts and lowers the otherwise Inextensible upper layer fluid columns. In the upwelling zone to the north of Ring center, the thermocline moves upwards and is hence flattened relative to the thermocline further north. South of Ring center, the local divergence from the mixed layer tends to deepen the thermocline. From Fig. VI.1.c, we see that both trends when applied to the warm Ring of Fig. VI.1.b compel the 'bowl' of isotherms to move to the south, suggesting that some fraction of meridional Ring migration is a forced phenomenon.

Stern (196.5) has shown that a uniform wind stress when interacting with a geostrophic eddy produces an Ekman pumping given by:

$$
w=-v \cdot(\underline{k x} \underline{\tau}) /(f+\zeta)
$$

where $\zeta$ is the geostrophic vorticity. The gradients in Ekman transport are caused by variations in the local rotation rate, $(f+\leq)$, and produce both up and downwelling centers. The resulting $w$ field is not unlike that depicted in Fig. VI.l and, as Stern pointed out, a west to east wind causes a warm eddy disturbance to translate southward. Note that the corrections to the undisturbed stress in the above formula are $O($ Ro). From Table VI.1, one notes the perturbations of the undisturbed stress caused by the temperature are of the scale of $|\underline{\tau}|=(.3$ to $.5 \times(7)$. Therefore, we neglect Stern's mechanism, as this scaling 
suggests it is smaller in effect than the present mechanism. It is interesting to note, however, that Stern's analysis of the effect of the divergence agrees with the present ideas.

\section{VI.C Governing Equations-}

The mixed layer buoyancy field is governed by:

$$
b_{t}+u b_{x}+v b_{y}=\left(\left(b_{1}-b\right) e+B_{0}\right) / h+k 2 b,
$$

where $b$ is mixed layer buoyancy, $b_{i}$ intermediate layer buoyancy, Bo surface buoyancy flux, $h$ mixed layer depth, $\mathrm{K}$ a coefficient of lateral diffusivity, and $u$ and $v$ horizontal velocities. It is interesting to compare horizontal heat advection to the entrainment flux of heat. In a Ring, the horizontal velocities are roughly $100 \mathrm{~cm} / \mathrm{sec}$ and have a scale of order $60 \mathrm{~km}$. Warm Ring mixed layers have been observed to deepen at rates of up to $3 \mathrm{~m} /$ day (Saunders, 1971), and according to Pollard, Rhines, and Thompson (1973), the density step scales as $\left(\tau^{2 /(}(2 \mathrm{~h} 2)\right)$. Hence, the comparison of these two terms ylelds a ratio:

$$
\mathrm{f} 2 \mathrm{~h}^{4} \mathrm{vby} /(\tau 2 \mathrm{ht})=10-81012102 /(6 \times 1063 \times 10-3)=5 \times 105 \gg 1,
$$

indicating that entrainment heat flux is weak and may be neglected. Similar comparisons allow us to simplify this equation further. Typical wind-driven velocities are roughly $1 \mathrm{~cm} / \mathrm{sec}$ and, compared to the geostrophic Ring velocities of $100 \mathrm{~cm} / \mathrm{sec}$, are negliglble. Surface heat fluxes, represented by $B_{0} / h$, are greater than entrainment heat flux:

$$
\text { Bohf } 2 /\left(h c^{2 h t}\right)=104 / 3 \gg 1,
$$

and while still apparently small compared to advective effects, represent the strongest non-conservative heat flux to the surface 
layer. Thus, at lowest order, the surface buoyancy equation becomes:

$$
b_{t}+J(\ddot{\psi}, b)=B_{0} / h+K \nabla^{2 b}, \quad E q \cdot V I \cdot 3
$$

where $\mathrm{J}$ is the Jacobian operator, and $₹$ the geostrophic streamfunction. As for the final term, there are few good estimates of $K$ appropriate to the mixed layer, therefore in the calculations to follow, we shall attempt to minimize its unfluence by using the smallest possible values. $\mathrm{K}^{\prime} \mathrm{s}$ presence is necessary however to insure numerical stability.

In the present experiments, Bo has been neglected as an influence on the surface buoyancy pattern. Ring SST cycles have been studied in Chapter IV, where it was shown that the strongest temperature contrasts occur in the winter, and that Ring mixed layers produce the variations in heat release necessary to affect the stability of the overlying air, and construct variations in stress. In the summer, SST contrasts weaken; therefore, stress should become more uniform. Specifying non-zero surface buoyancy contrasts and allowing them to evolve according to Eq. VI. 3 with $B_{0}=0$ more resembles and is meant as a model of wintertime Ring. SST. The intial shape assigned to the buoyancy field will be taken from the tracer distributions computed in Chapter III. Ekman divergences based on these SST fields should produce evolution suggestive of wintertime Rings. This approximation gains much in terms of reduced computing needs. By neglecting surface buoyancy flux, it is evident from Eq. VI.3 that we no longer have to compute mixed layer depth, ostensibly reducing the number of dependent variables from three to two. In fact, the reduction is better as the equation 
governing mixed layer depth requires the knowledge of at least one more variable (usually interior buoyancy).

The divergence of the upper layer mass transport provides the dynamic link of the surface and the interior. The Ekman divergence, Eq. VI.2, becomes:

$$
w_{e}=j\left(\tau_{(y)} b_{x}-\tau_{(x)} b_{y}\right) /\left(f_{o g} \uparrow\right), \quad \text { Eq. VI.4 }
$$

where we have used a IInear equation of state of the form:

$$
P=P_{0}\left(1-P\left(T-T_{0}\right)\right) \quad \text { Eq. VI.5 }
$$

to convert temperature to buoyancy and assumed the air temperature constant. In Eq. VI.4:

$$
\begin{aligned}
& \tau_{(x)}=\hat{C}_{d_{0}\left|\underline{u}_{a}\right| u_{a},} \\
& \tau(y)=\hat{C}_{d_{0}}\left|\underline{u}_{a}\right| v_{a},
\end{aligned}
$$

and $g$ is gravity. In the present experiments, $\tau_{(y)}=0$.

The interior will be governed by the equivalent barotropic equation:

$\left(\nabla 2-r^{2}\right) \alpha_{t}+\left(f_{0}\right)-1 \xi_{111 J}\left(\alpha,\left(\nabla^{2}-r^{2}\right) \alpha\right)+\alpha_{x}=f_{0}{ }^{2} F(0) w_{e} / H \quad$ Eq.II. 14

where $\Gamma^{2}$ is the elgenvalue corresponding to the first elgenmode, $F(0)$ the surface value of the baroclinic eigenmode, $H$ the depth of the fluid, and $\Sigma_{111}$ the baroclinic self interaction coefficient. Note that the expressions for the surface velocities of Eq. VI.3 are:

$$
u=-F(0)\left(\alpha_{y}\right) / f_{0},
$$

and:

$$
v=F(0)\left(\alpha_{x}\right) / f_{0} \text {. }
$$

The nonlinearity of the problem, i.e. the connection between the transport of the surface waters and the forcing of the interior, is 
apparent from Eqs. VI.3, 4, and 7. As such, we have no way of linearizing the equations and retaining the correct physical processes, and for this reason, no analytical solutions to this set have yet been found. It is, however, somewhat enlightening to consider the linear evolution of an initially motionless ocean, possessing a surface temperature field, which is subjected to the onset of an eastward wind. Under these assumptions, the mixed layer will translate southward at the Ekman velocity, advecting with it the surface temperature structure, and therefore the horizontal divergence:

$$
w_{e}=w_{e}\left(x, y-\tau(x) t /\left(f_{o h o}\right)\right) .
$$

To the interior, the pumping resembles a translating wind stress pattern. The related Rossby wave excitation problem is well known (Flierl, 1978), from which we expect the forcing to resonate with those planetary waves whose phase speed matches the Ekman drift. The interesting point of this problem is that an otherwise constant wind can interact with a surface temperature distribution to produce interior motions.

VI. d. Numerical Results-

The non-dimensional set of equations which describe the Ring evolution are:

$$
(\nabla 2-172) u_{t}+Q \xi_{111} J(\alpha,(-2--2) \alpha)+\alpha_{x}=-b_{y}+k v \sigma_{\alpha},
$$

and:

Eq. VI. 6

$$
\mathrm{bt}_{t}+\mathrm{QF}(\mathrm{O}) \mathrm{J}(\mathrm{c}, \mathrm{b}, \mathrm{b})=\mathrm{Kb} \nabla 2 \mathrm{~b},
$$

where $Q=U /(\beta L 2)$, a measure of the nonlinearity of the flow, $X=-F(0) j T_{o b o} /(g H t<L U)$, and we have employed a biharmonic friction with 
coefficient $K$. Notice that for a west to east wind, $X$ is negative. Typical values of all the parameters and scales are listed in Table VI.2. The values of $F(0), \xi_{111}$, and $\Gamma 2$ computed by Flierl (1978) from the MODE buoyancy profile were used and the remainder of the scales were chosen to agree with those of McWilliams and Flierl. Here $\mathrm{K}=5 \times 10-4$; there is currently no justification for this choice, other than it is 'small'. The results of the computations to be discussed in this chapter were largely unaffected by a reduction of $K$ to $5 \times 10^{-5}$.

In Fig. VI.2, we compare the results of two experiments which will be referred to as expOb and explb. ExpOb is a control experiment in which we have turned off the Ekman pumping, i.e. set $j=0$, and allowed the Ring to evolve freely. In explb, the value of the coupling constant $j$ was set to . 1 . The other numerical parameters are listed in the figure caption. The initial conditions for both experiments, contained In the panels a and $d$, were chosen as Gaussians of e-folding scales 60 $\mathrm{km}$, for the baroclinic amplitude, and $90 \mathrm{~km}$, for the surface buoyancy field.

Clearly, the shapes of the sea surface temperature field and the interior pressure field compare favorably between the two experiments. By day 60 , however, we see evidences that the pressure field in explb is travelling at a different rate than that in expob. Both of the above observations are reinforced by the graphs of Fig. VI.3 and 4 in which the histories of the maxima in pressure and the path of the Ring are compared. The numerically generated positions of the local pressure maxima, which are graphed in Fig. VI.4, are listed in Table VI.3. From 

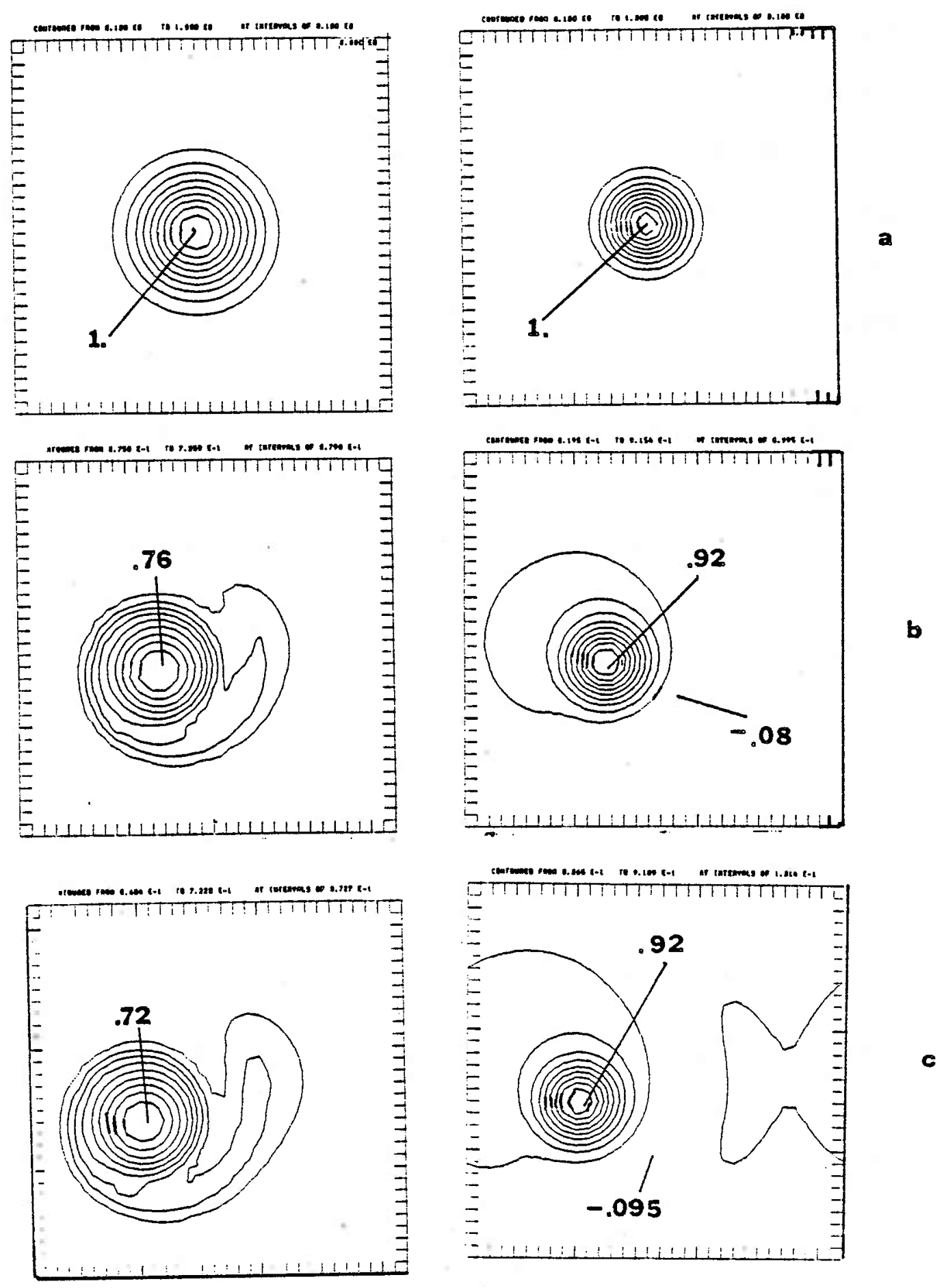

Fig. VI. 2

continued next page 

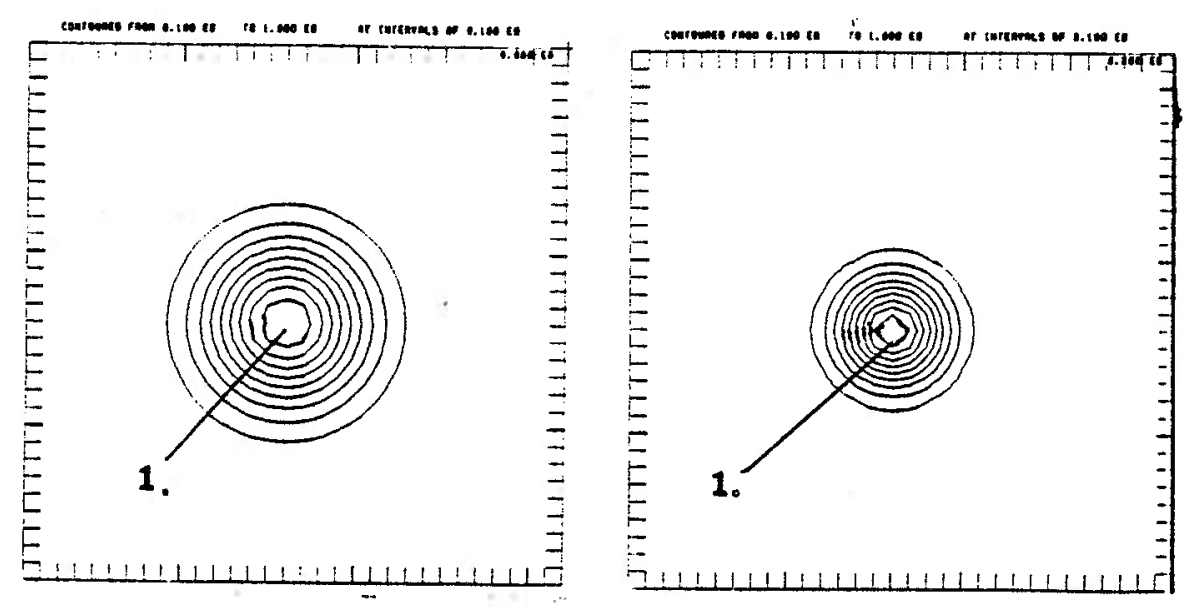

d
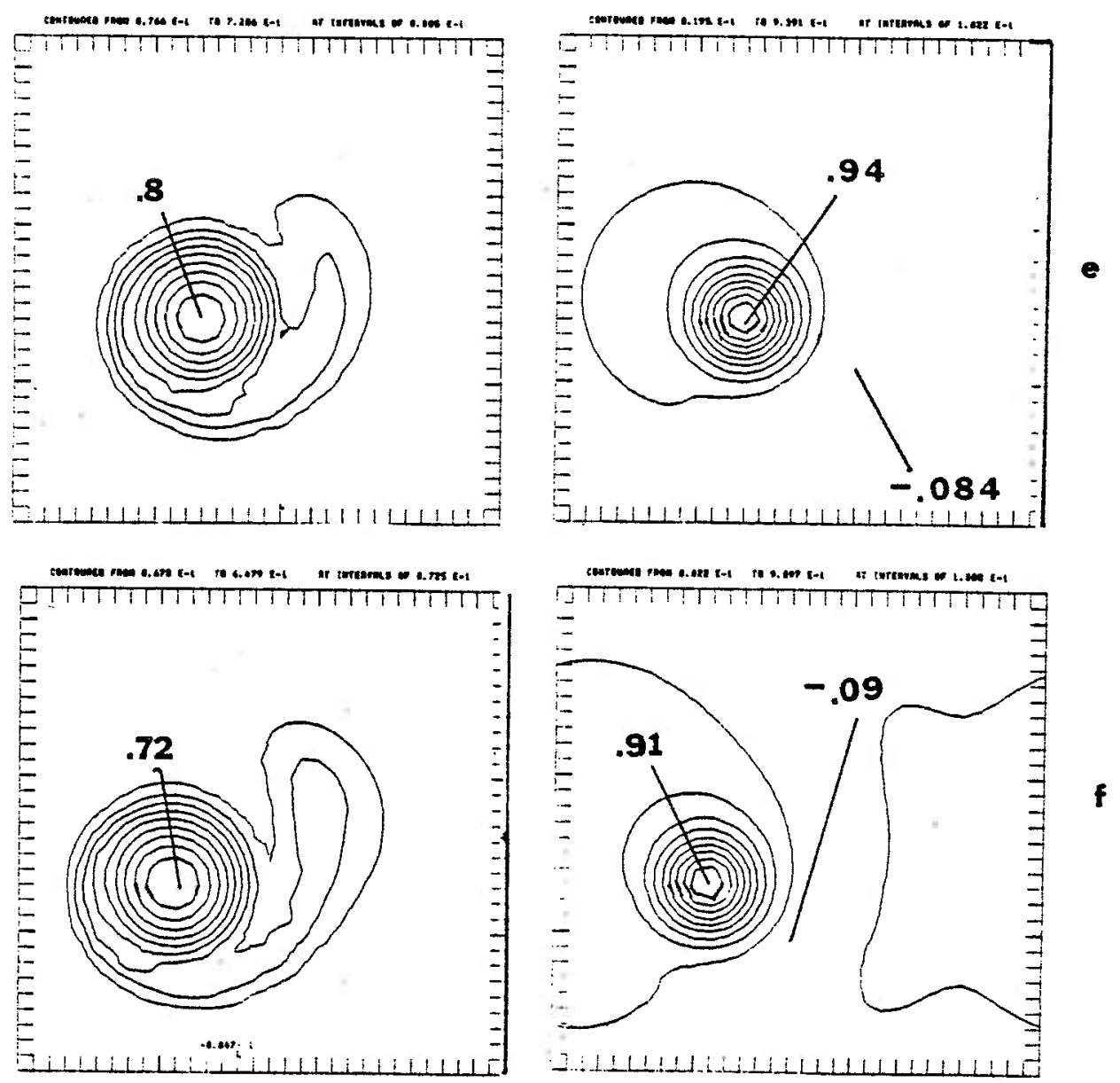

Fig VI.2. Forced Ring Evolution

Plotted are the baroclinic horizontal amplitude functions and the sea surface temperatures from expob, after (a) 0 days, (b) 40 days, and

(c) 60 days. In this set, $Q=4.76, \xi_{111}=2.1, K=5 \times 10^{-4}, \Gamma 2=2$, and the coupling coefficient, $j$, was set to 0 . In the second set, the plots are from explb, with all parameters the same except for $j$, which is set to.. Again, the plots are for (d) 0 days, (e) 40 days, and (f) 60 


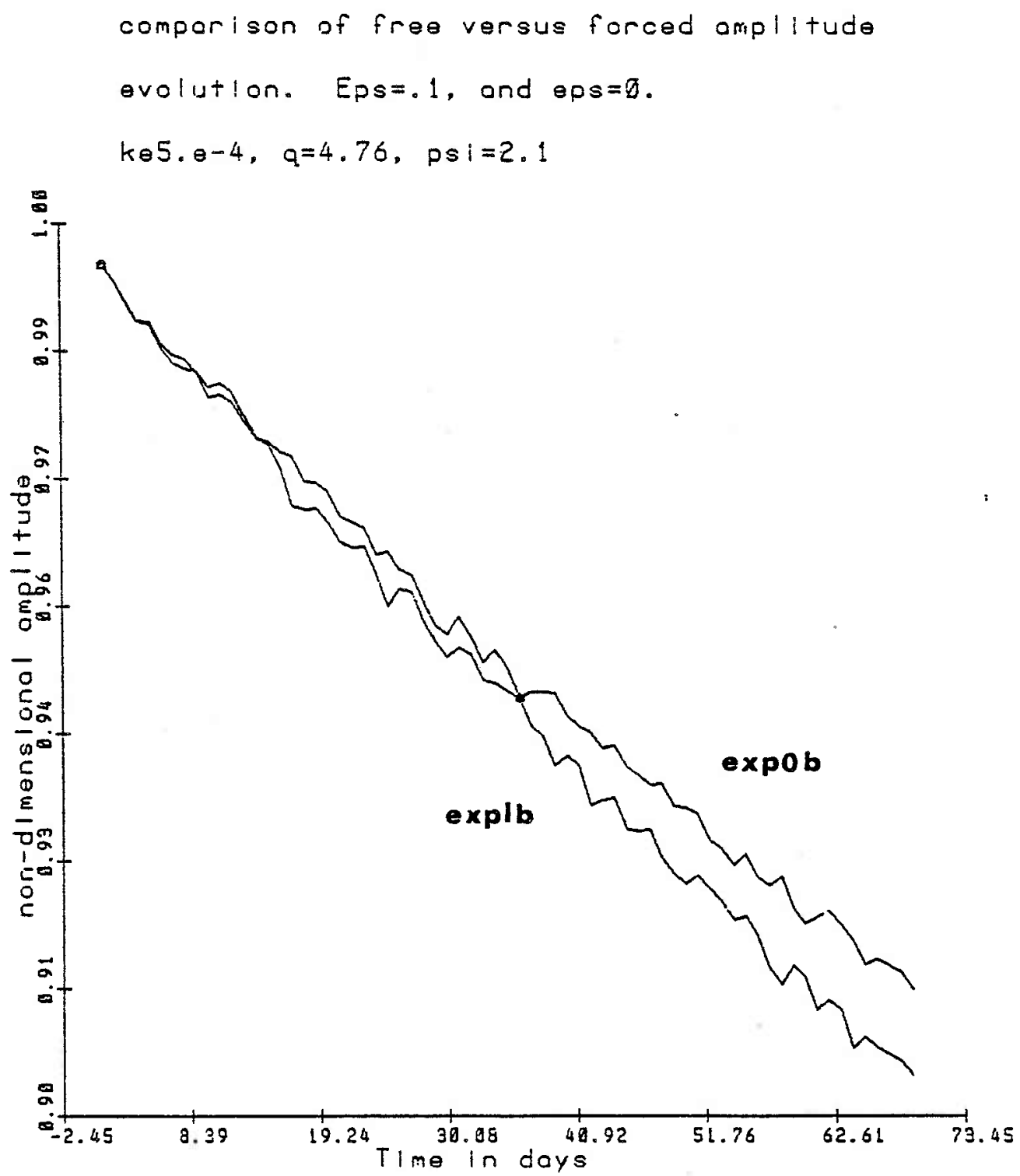

Figure VI.3. A Comparison of Ring Amplitudes

Here we compare time histories of the maximum pressure amplitudes from expOb and explb. Note that the amplitude evolution is largely unaffected by the forcing; after 70 days, the difference in the thermocline depression is $0(5 \mathrm{~m})$. The wiggles in the plot are due to the uncertainty involved in the location of the maximum. 


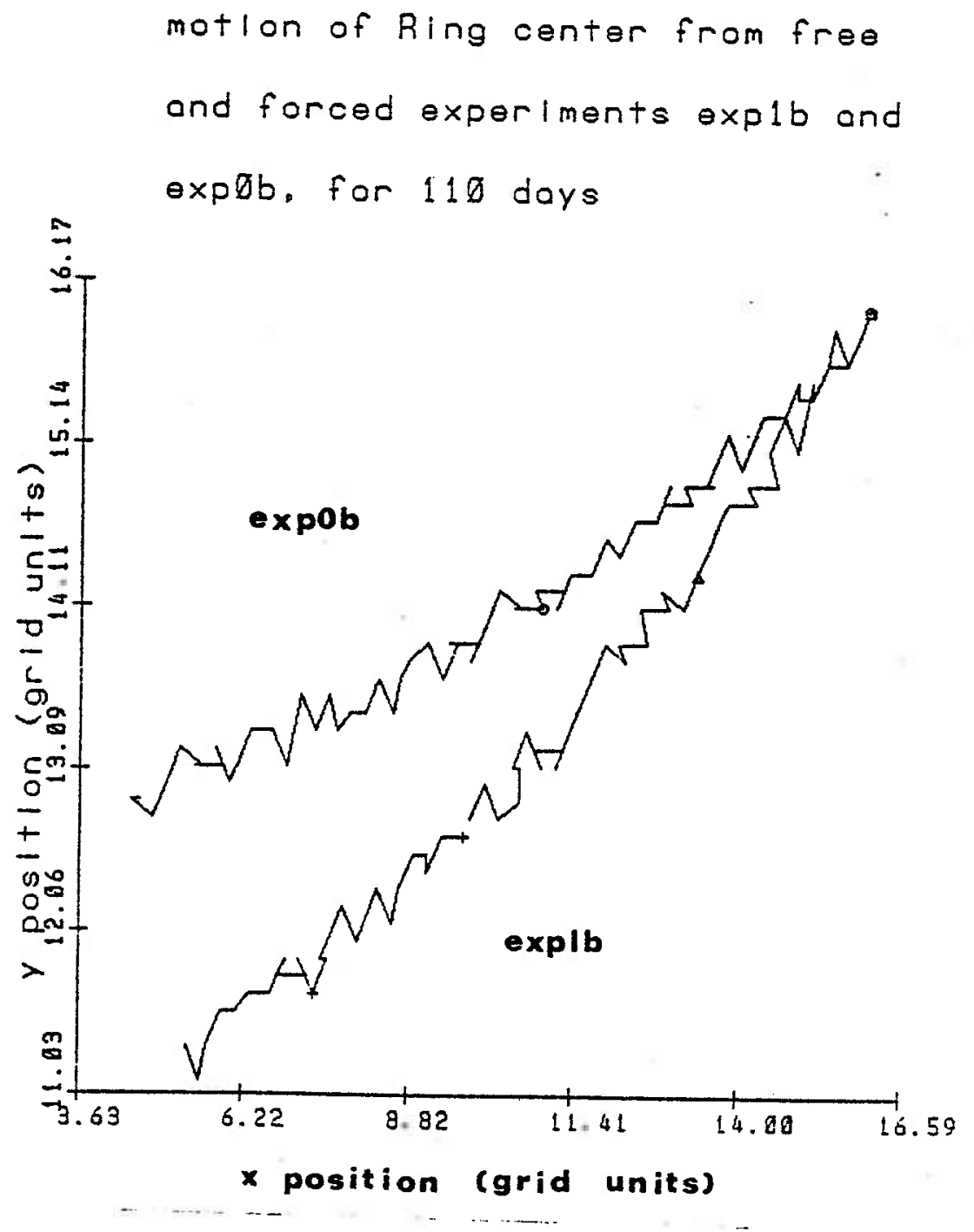

Figure VI.4. Forced and Free Ring Trajectories

Trajectorles of Ring center are plotted from expOb and explb. Ring center was defined as the location of the pressure maximum. Notice the decreased westward drift and increased southward drift in the forced experiment. All locations are to an accuracy of $4 \mathrm{~km}$. 
Table VI. 3

Locations of the Local Maximum of Pressure versus Time

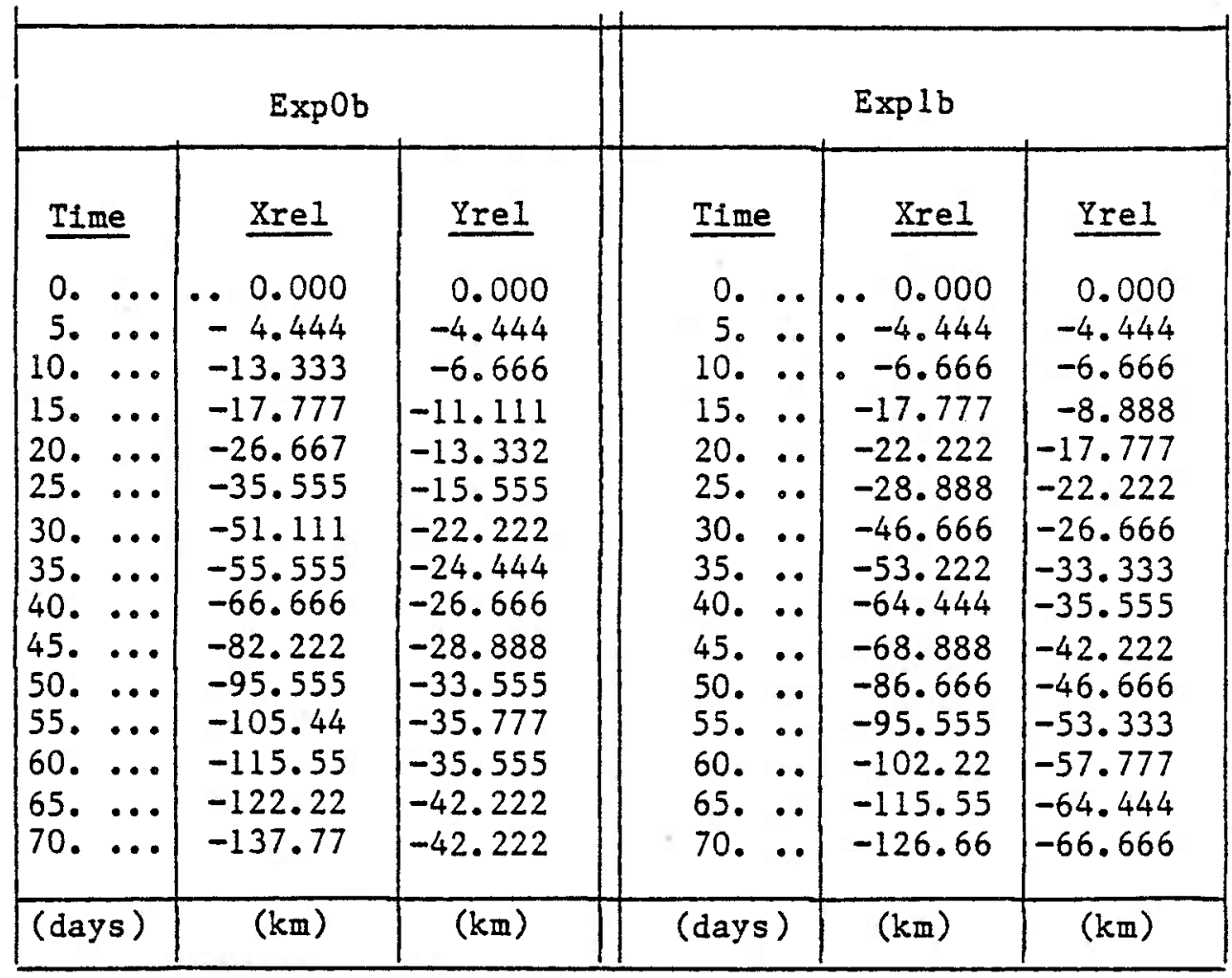

All distances measured relative to initial center. 
the first graph, we see that the amplitude decay is nearly identical in the experiments; therefore, we conclude that, unlike the results of the previous chapter, the induced pumping is not seriously affecting the rate at which the Ring is losing energy. From Table VI.3, we calculate an average propagation velocity for the maximum in pressure of ( -2 $\mathrm{km} /$ day, $-1 \mathrm{~km} /$ day) in explb, and $(-2.4 \mathrm{~km} /$ day, $-.5 \mathrm{~km} / \mathrm{day})$ in expob. The forced Ring of explb is moving to the south at nearly twice the speed of the $\mathrm{Ring}$ in expOb, but to the west at a lesser rate.

Finally, note from the plots in Fig. VI.2 that the pressure fields develop azimuthally dependent structures, which consist mostly of a high pressure center west of each Ring and a low pressure center to the east. The generation of those structures has been commented on by McWilliams and Flierl and is due to dispersion from the initial, isolated high. Both centers remain coherent with the Ring throughout the experiments.

Comparing the locations of the high and low pressure centers from each experiment, we notice they are somewhat different. Relative to those in expOb, the local centers in explb are rotated in a counterclockwise sense about the Ring, a difference which becomes more pronounced for later times. However, the amplitudes of each of the centers differs by only a few percent. For example, the low pressure of exp0b at day 40 is -.08 , compared to that of explb which is -.084 . 
Table VI. 4 Experiments and Parameter Settings

\begin{tabular}{|c|c|c|c|c|c|c|}
\hline Experiment & comment & $\begin{array}{c}F \\
n-d\end{array}$ & $\underset{n-d}{k}$ & $\begin{array}{l}l_{b} \\
k m\end{array}$ & $1^{j} /{ }^{\circ} \mathrm{C}$ & $\begin{array}{l}\operatorname{mpr} \\
\mathrm{km} / \text { day }\end{array}$ \\
\hline $\exp 0 b^{*}$ & warm & 3 & .04 & 90 & 0 & -.5 \\
\hline $\exp 1 b$ & warm & 3 & .04 & 90 & .1 & -1 \\
\hline $\exp 10 b$ & $\operatorname{cold}$ & 3 & .04 & 90 & .1 & .3 \\
\hline $\exp 11 b$ & warm & 1 & .04 & 60 & .1 & -.7 \\
\hline $\exp 12 b$ & warm & 3 & .4 & 90 & .1 & -1 \\
\hline
\end{tabular}

In all experiments, the time step was .02 of a day, and the spatial increment was $20 \mathrm{~km}$. All began with an initial condition in pressure of a Gaussian with length scale $60 \mathrm{~km}$. The temperature initial condition was a Gaussian of scale $I_{b}$. The coupling coefficient was $j$, and $F$ measured the surface intensification of the Ring. $\mathrm{K}$ is the lateral surface temperature coefficient of diffusivity. 'mpr' stands for meridional propagation rate.

\footnotetext{
*

Note that we can obtain the motion of a freely evolving cold Ring from this experiment. The governing equation is invariant to the transformation $(\alpha, y)(-\alpha,-y)$, so warm Ring simulations become cold Ring simulations with a reverse of north and south and the sign of the pressure. Therefore, freely evolving cold Rings move north at a rate of $.5 \mathrm{~km} /$ day.
} 
Parameter Studies-

Three other experiments were conducted in order to test a range of Ring types (see Table VI.4).

ExplOb was designed as a cold core Ring experiment, with a central Low pressure covered by a cold pool. SST contrasts across cold Rings are generally weak (Chapter IV, and Vastano, Schmitz, and Hagan, 1980), and in explob, we have reduced the size of the temperature contrast to $2.5{ }^{\circ} \mathrm{C}$ (in the others, $\delta \mathrm{T}$ was $5{ }^{\circ} \mathrm{C}$ ). The nonlinear interactions of freely evolving cold Rings with their dispersion field move them northward, (McWllliams and Flierl, 1979), and by the inclusion of the forcing, this tendency has been reduced. The forced Ring of expl0b moves northward at a rate of $.3 \mathrm{~km} /$ day, as compared to the meridional motion of the free Ring of $.5 \mathrm{~km} /$ day.

In expllb, we have reduced the area covered by the warm surface pool by choosing an initial Gaussian of $60 \mathrm{~km}$ length scale. In addition, the size of the surface trapped zone has been reduced from that in explb by setting $F(0)$ to 1 . The results indicate a dependence of the propagation on these parameters, as southward Ring motion in $\operatorname{expllb}$ as been reduced from $-1 . \mathrm{km} /$ day, to $-.7 \mathrm{~km} / \mathrm{day}$.

Finally, in expl2b, we set the value of the lateral coefficient of heat diffusivity to .4 , and noticed no significant change in propagation or Ring evolution from that in explb. 
In all experiments, the pressure and SST fields evolved in such a way that they visually resembled those in Fig. VI.2, with the only major change being a reduction in the size of the SST pattern when the size of the trapped zone was reduced. Therefore, Figs. VI.2 are representative of the pressure and SST configurations and extrema for all tests. It would be interesting to expand the set of experiments by more fully exploring the possible combinations of trapped zone size and initial buoyancy structure.

VI.e. Discussion-

Both warm and cold Rings are observed to propagate southwestward, with a meridional component of up to $-1.5 \mathrm{~km} /$ day (Richardson, Cheney, and Worthington, 1978). Why they move south is currently unknown, although understanding the trajectory of a Ring is important to evaluating their influence on the surrounding waters. Mean state advection has been suggested as a mechanism for southward Ring motion because cold Ring motion agrees with the sense of the general circulation (the Ring Group, 1981). Whether this is true for warm Rings is less clear. From the work of Meid and Lindemann and McWilliams and Flierl, we know model Rings can self-propel, with cold Rings moving north and warm south. Topography is also a possibly important steering mechanism, probably playing a stronger role for warm Rings than cold. According to the results here, we apparently have another mechanism for producing southward motion and we will now investigate how it is produced, and its magnitude. 
Intregral Constraints-

From integral constraints, McWilliams and Flierl were able to demonstrate that the departures of their freely evolving Ring from a strictly radial configuration were responsible for much of its propagation. Similar arguments prove enlightening in the present case. The center of mass (COM) vector of $\alpha, \underline{X}=(X, Y)$ is defined as:

$$
\underline{X}=\iint \underline{x} \times d A /\left(\iint \alpha \mathrm{dA}\right) . \quad \text { Eq. VI.7 }
$$

Predictive equations for both components of $\underline{X}$ may be obtained from Eq. VI.6 with the assumptions that both $\alpha$ and $b$ vanish at infinity:

$$
x_{t}=-1 / \Gamma^{2}
$$

and:

$$
Y_{t}=\alpha\left(\int b d A\right) /\left(\left(\int \alpha d A\right) \Gamma^{2}\right),
$$

where we have used we from Eq. VI.4. Note that the zonal center of mass, $X$, moves at the long Rossby wave phase speed, a result which applies in the absence of pumping. The $y$ center of mass experiences a net meridional drift which is, quite evidently, a forced response. Contrast this with unforced evolution, where $Y_{t}=0$.

Note the sign of $Y_{t}$ depends on the relative sign of the average buoyancy with respect to the average baroclinic amplitude. Since $R$ is negative, a warm pool overlying a solitary high pressure center forces $Y$ to the south. The same southward tendency holds for a cold pool over low pressure. Both of these SST-baroclinic pressure configurations describe Rings, the former warm core and the latter cold core. 
Note from Table VI.3 that the propagation speed of the pressure maximum in explb is not that prescribed by Eq. VI.8, and the discrepancy can be shown to be related to the amount of baroclinic 'mass' which has been radiated by the Ring. The difference between $X$ and Ring center

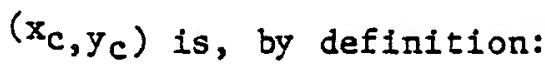

$$
x-x_{c}=\iint\left(x-x_{c}\right) \alpha d A /\left(\int \alpha d A\right) \text {, }
$$

and:

Eq. VI.9

$$
Y-y_{c}=\|\left(y-y_{c}\right) \alpha d A /\left(\int \alpha \cdot d A\right) \text {. }
$$

Clearly, if $\alpha$ were radially symmetric, the above expressions would reduce to zero. The two radially asymmetric features in $\alpha$ of greatest magnitude are the leading high and the trailing low; hence, their presence is having a profound influence on Ring propagation. Again following McWilliams and Flierl, we define a 'departure field', $\alpha^{\prime}$, of the baroclinic streamfunction amplitude from a purely radial configuration:

$$
\alpha^{\prime}=\alpha-\alpha(r)=\alpha-\alpha_{c} e^{-\left[\left(x-x_{c}\right)^{2}+\left(y-y_{c}\right)^{2}\right]},
$$

where, in the second equality, we have chosen a Gaussian based on local maximum pressure, $\alpha_{c}$, for the radial function. In terms of $\alpha^{\prime}$, Eqs. VI. 9 become:

and:

$$
x-x_{c}=-\|\left(\left(x-x_{c}\right) \alpha x^{\prime} d A / \iiint d A\right)
$$

$$
Y-y_{c}=-\iint\left(y-y_{c}\right) \alpha^{\prime} d A /\left(\iint \alpha \cdot d A\right),
$$

Eq. VI. 10

which demonstrates the importance of the departure field. Leading highs and/or trailing lows cause the Ring center to lag behind the zonal COM. Similarly, northerly highs in conjunction with southerly lows produce a net southward Ring displacement relative to $Y$. Note that Eq. VI.12 is independent of the dynamics, and hence the dispersion field has the 


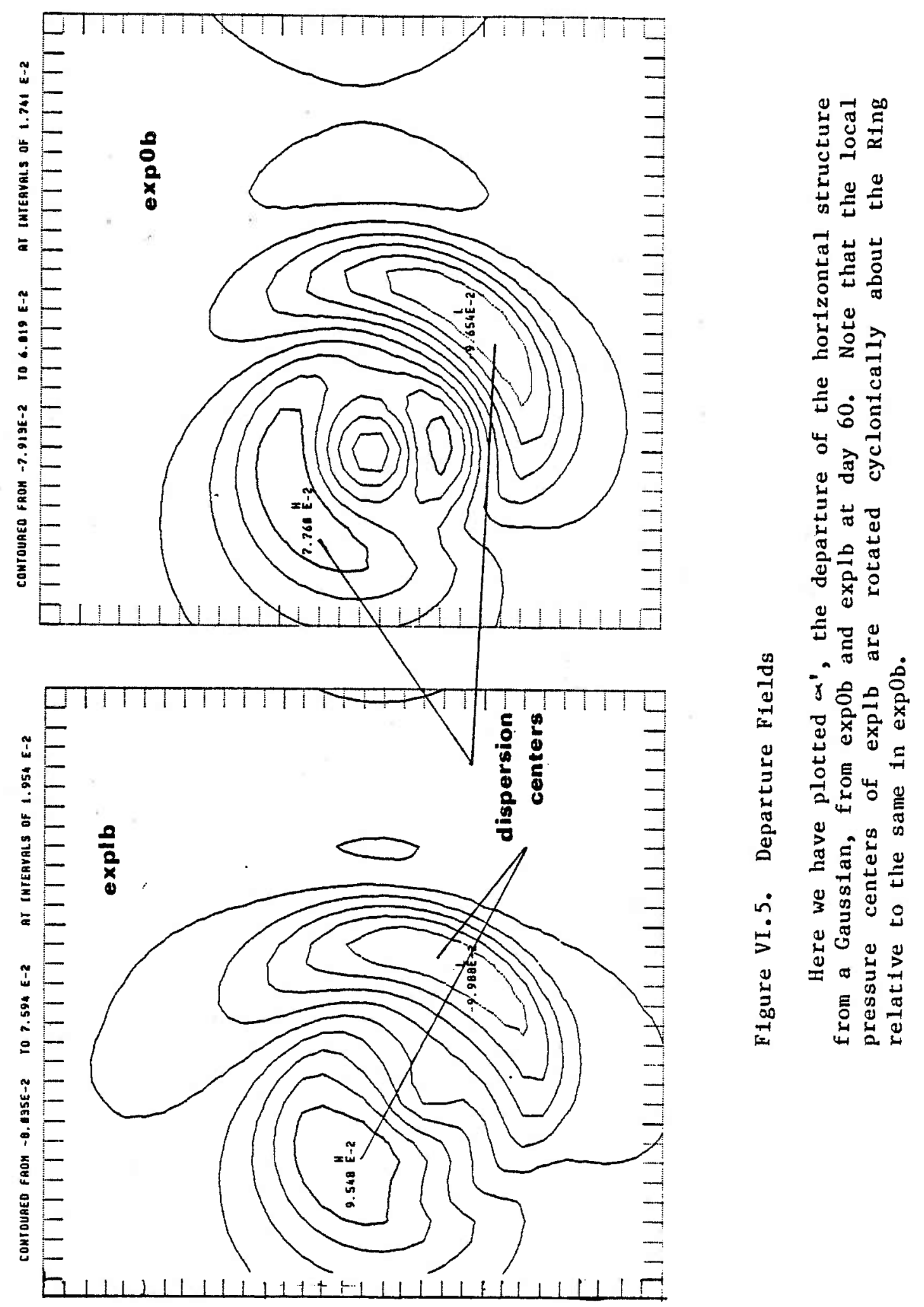


above effect on the Ring regardless of the existence of forcing. The integral constraints in Eq. VI.10 convey only the kinematic tendencies of the departure field. The clockwise rotation of a high pressure center tends to push eastern fluid to the south and southern fluid to the west, and vice-versa for a low. The above integrals measure the effect that the departure field is imparting to the Ring.

In Fig. VI.5, we plot departure fields for both expOb and explb. The important distinction therein concerns the relative positions of the local extrema. As noted in section $c$, the low and the high in Explb are rotated cyclonically about Ring center relative to the same in expOb.

Zonal Propagation-

For the duration of the experiments, the high pressure of $\alpha$ is to the west and the low pressure to the east of Ring center. Therefore, we expect both Rings to lag behind zonal COM, although the free Ring of expOb eventually moves west faster than its counterpart in explb (see Table VI.3). The acceleration of the free Ring was first noticed by McWilliams and Flierl who pointed out that the zonal propagation speed of the free Ring appeared to be asymptoting to the phase speed of a long Rossby wave. This was interpreted to be a result of the slow anticyclonic motion of the dispersion centers about the Ring, which stopped just short of lining them up meridionally with Ring center. Clearly, as the centers move about the Ring, the zonal weight assigned to either the leading high or the trailing low diminishes, causing the Ring to accelerate. In explb, the tendency for the rotation of the centers is 
decreased, so that the local maxima of each center remains nearly stationary, and are unable, through their circulations, to augment Ring zonal propagation. Thus the westward pattern speed of the forced Ring is less than that of the free.

Meridional Propagation-

By an area average of the amplitude and buoyancy equations, we see:

$$
\left(\int(b d A)_{t}=0\right.
$$

and:

$$
\left(\iint \alpha d\right)_{t}=0
$$

so that the forced meridional COM propagation in Eq. VI.10 can be computed from the initial conditions. For explb, this becomes:

$$
Y t=98 / 8 \text {, }
$$

or dimensionally:

$$
Y t=-.6 \mathrm{~km} / \mathrm{day},
$$

which should be compared with the computed Ring center propagation of $-1.0 \mathrm{~km} /$ day. Thus, we conclude that roughly one-half of the meridional migration of the numerical Ring is due to the forcing. The difference between the motion of the Ring center and that of the COM is $-.4 \mathrm{~km} / \mathrm{day}$, and is due to the dispersion field. Note that the magnitude of this drift is roughly the same as that in expob, in agreement with the observation that the strength of the dispersion field in both experiments is comparable. Given the crudeness with which we have modeled the temperature dependence of the coefficient of drag, we can not say much more than the forced southward migration of the Ring is on the order of $.5 \mathrm{~km} /$ day; however, this is the same order as the 
meridional component of the general circulation and the self-induced meridional propagation due to Ring-dispersion field nonlinear interaction. The important point is that the present mechanism is large enough in its effects to account for a significant fraction of meridional Ring motion.

VI.f Potential Vorticity Budgets-

The novel Ring propagation in explb is a direct result of the forcing, which must therefore affect the Ring's potential vorticity. Consider the potential vorticity (q) budget of a particle, outside of the trapped zone, about to interact with a Ring.

In the following examination of $q$, we will use expllb. In this experiment, $j=.1$ and, with the exception of $F(0)$, all other parameters matched those in explb. In expllb, $F(0)$ was set to 1 , rather than 3 . This has the effect of decreasing the trapped zone size, owing to the reduced surface velocites, which are computed according to:

$$
\begin{aligned}
& u=-F(0) \alpha_{x}, \text { and } \\
& v=F(0) \alpha_{y} \cdot
\end{aligned}
$$

The initial conditions for $\alpha$ in explib were the same as in explb, a Gaussian of length scale $60 \mathrm{~km}$. The SST condition was also chosen as a Gaussian of scale $60 \mathrm{~km}$, which is a reduction from the $90 \mathrm{~km}$ Gaussian used in explb. As a result, the forced component of meridional Ring migration was reduced from $-.6 \mathrm{~km} /$ day to roughly $-.3 \mathrm{~km} / \mathrm{day}$, and the total Ring migration from -1 . $\mathrm{km} /$ day to $-.7 \mathrm{~km} / \mathrm{day}$. This still constitutes a sizeable increase in southward Ring migration over the 
vortico! volocity dus to toreing, from explib.dot. $F(\theta)=1$.

Ting in doys $=39+1$

CONTOURED FROH -3.43LE-2 TO 4.476 E-2 AT INTEAYRLS OF 0.879 E-2

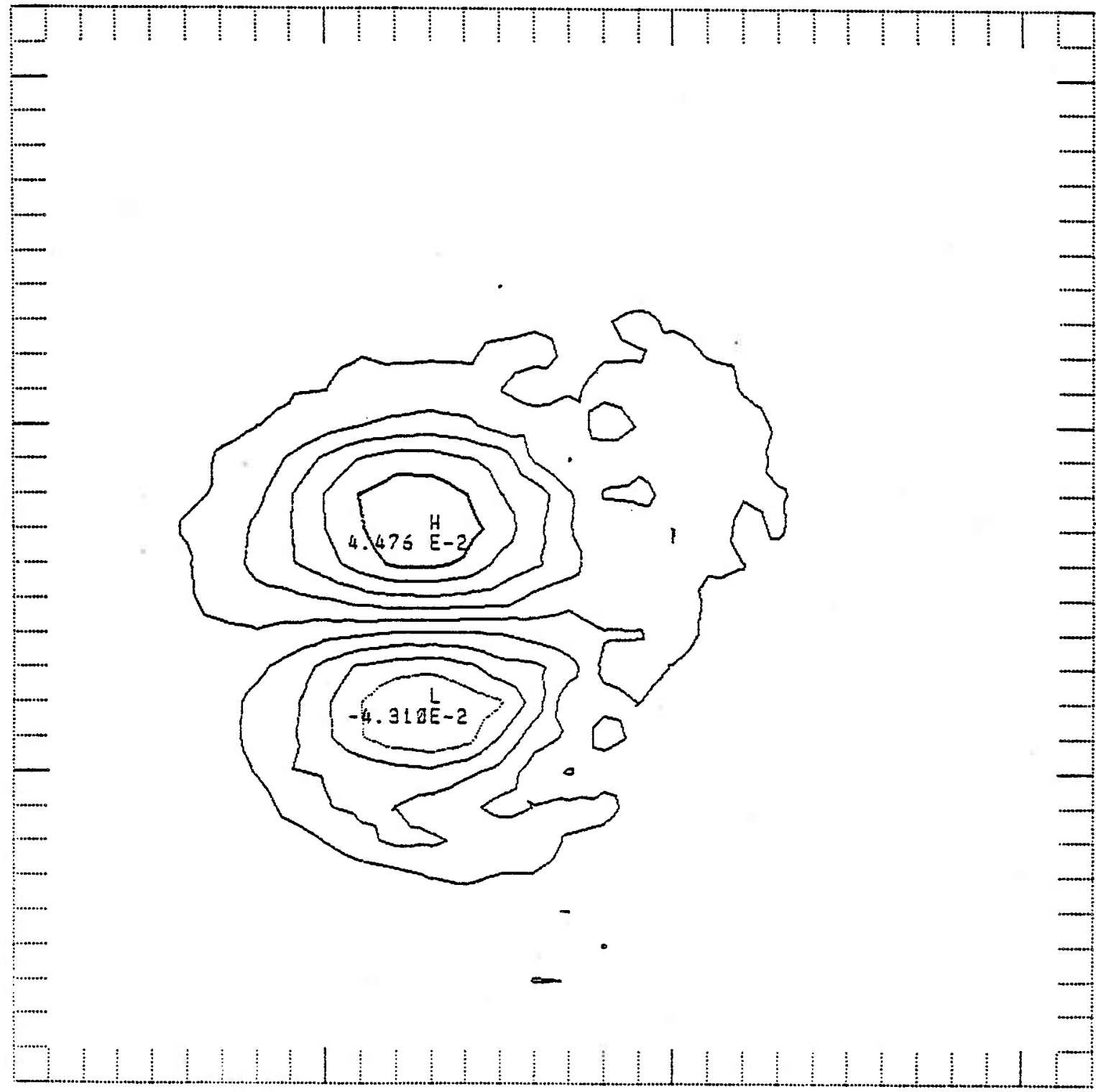

Figure VI.6. Forced Divergence

Here we have plotted a graph of the Ekman divergence produced by the surface temperature field at day 40 from expllb. In this experiment, $\Gamma^{2}=2, K=5 \times 10^{-4}, \xi_{111}=2.1, Q=4.76, F(0)=1$, and $K b=.04$. Note the regions of upwelling to the north and downwelling to the south. 
unforced speed of $-.5 \mathrm{~km} /$ day, and indicates that the results of this chapter are not overly sensitive to parameter variations.

In F1g. VI.6, we plot a map of the Ekman divergence computed according to Eq. VI.4. Although the pumping intensifies behind the Ring, in the 'tail' emanating from the trapped zone, the largest centers of vertical velocity lie over the Ring thermocline expression. Note that the northern half of the Ring experiences upwelling and the southern half downwelling.

Ekman divergence can produce potential vorticity through vortex tube stretching or relative vorticity generation:

$$
q_{t}=\left[\nabla^{2}-r^{2}\right] \alpha_{t}
$$

From we, we can compute the forced trend in amplitude production, $\alpha_{t}$, by solving the elliptic equation:

$$
[\nabla 2-r 2] \psi_{t}=-\dot{x} b y \text {. }
$$

The generation of vortex tube stretching is given by:

$$
-i^{2}<t
$$

which may then be combined with we to compute the forced production of relative vorticity:

$$
\nu^{2} \alpha_{t}=-\jmath b_{b y}+\Gamma^{2} \alpha_{t}
$$

Plots of these fields are contained in Fig. VI.7. Note that the production of $q$ is equally divided between these two components. The principle reason for this comes from the near match of the Rossby Deformation Radius, $\Gamma^{-1}$, and the scale of the pumping (cf Fig. VI.6). 

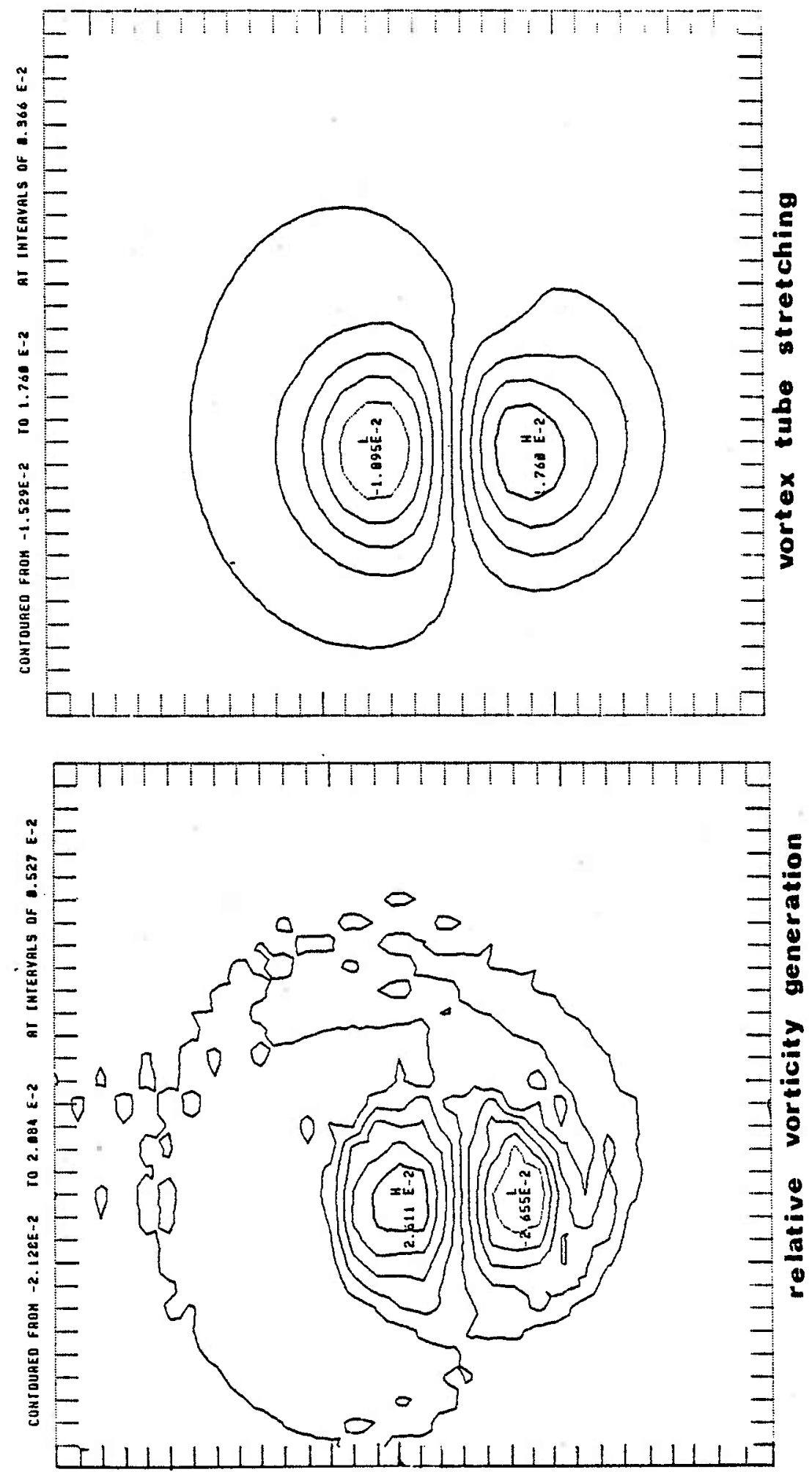

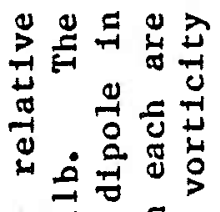

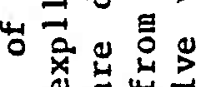
ร ब

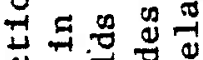
可

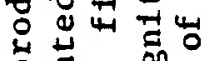

है

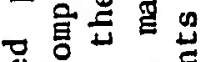
نู

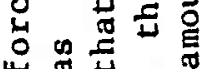
क व

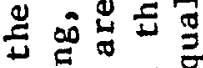
岁艺墕 过

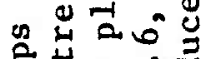
震落步宫 उ 过 出园武焉 엄 $x$ 앤 a.

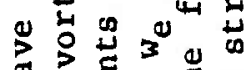
胥 $>3$

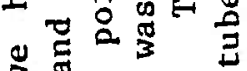

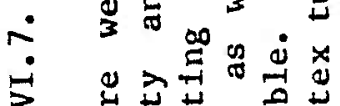

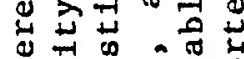

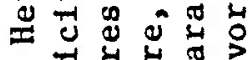
吉岕引苔 苍志导导 
If the Ring were not forced, 1.e. $w_{e}=0$, parcels moving north around the Ring, in order to conserve potentlal vorticlty, would develop negative relative vorticity to cancel the increase of $q$ due to beta. In terms of the pressure field, this is equivalent to the production of a high, which is how the local maximum to the northwest of the freely evolving Ring is maintained. Now consider the alteration of the high pressure center caused by the forcing. As a fluid column moves from the southwest of the forced Ring towards the north, it initially receives mass from the boundary layer. The column responds by thickening vertically and expanding horizontally. The Coriolis force in conjunction with the diverging radial velocities of the column produces negative relative vorticity, and the vertical thickening presses the thermocline. Both of these forced responses augment the production of a high. To the north of Ring center, the columns lose mass to the boundary layer, part of which is supplied by a horizontal convergence in the interior and the rest by vortex tube shrinkage. The thermocline responds to the shrinkage by uplifting and the fluid responds to the convergence by positive relative vorticity production; both of which counter the production of a high. In total, the location of the high to the west of the Ring is shifted to the south of its unforced position.

Similar arguments apply to the trailing low. The effect of upwelling on a parcel is to increase its potential vorticity, while that of downwelling is to decrease it. Therefore, for particles moving south, the forcing tends Initially to compensate for the loss of $q$ due to beta. Once south of Ring center however, the forcing changes sign and supplements the loss of $q$. For a southbound column, the development 
of the low is first enhanced and then suppressed. To the observer in a stationary frame, the location of the minimum is shifted to the north.

Note that the above forced trends in pressure, highs shifting south and lows north, are precisely the evolution of the interior which results in the southward shift of the center of mass (cf. Eq. VI.10). Similar arguments describe the potential vorticity budgets and the associated drifts of the Ring trapped zone.

Again consider a particle in a clockwise transit, but within the trapped region. In the northern half of the Ring, as the particle is moving west, the mass flux is directed up into the boundary layer (Fig. VI.6). As a result, a column of fluid is both stretched, creating negative relative vorticity, and lifted, which upwells the deep thermocline. In the south, the mass flux into the interior locally depresses the thermocline and creates negative relative vorticity. With each succeeding pass of a column, the thermocline is flattened to the north and deepened to the south; therefore the pressure pattern shifts to the south.

VI.g. Summary-

In this chapter, we have demonstrated that the temperature dependence of the coefficient of drag in the formula for wind stress results in a forced propagation of Gulf Stream Rings. The large temperature contrasts associated with Rings produce regions of enhanced and suppressed wind stress and creates both convergent and divergent 
flow. For warm and cold core Rings, the response of the Ring is to move to the south.

From Chapter IV, we anticipate that the strength of the forced motion of cold core Rings will be less than that of warm core, owing largely to the reduced SST contrasts. Nonetheless, the forced propagation of a cold core Ring will be in opposition to that caused by the departure field. (Recall that the freely evolving warm core Ring simulations presented here may be immediately extrapolated to cold core Rings by substituting low pressure for high and north for south (cf. Eq. VI. 6). This is not so if we include the forcing term, $\left.\gamma b_{y}.\right)$ In one experiment using a cold core Ring and a weak SST contrast, the resulting northward motion of the Ring was reduced by $\approx 50 \%$, but not reversed. There are several mechanisms which potentially affect Ring migration, such as mean advection and self-propagation, and in this chapter we suggest that forced migration is as large as either. Warm Rings are possibly moved southward by all three. For cold rings, it is interesting to speculate that their southward drift is a resultant of all three. Cold ring northward self-propagation is about the same size as southward mean advection and the remaining forced tendency could account for observed motion.

The ability of Rings to transport fluid is essential to their systematic, forced, meridional drift. The forcing moves the Ring south, which in turn shifts the trapped zone. The movement of the trapped zone transports the temperature field which is responsible for the creation 
of the pumping. The system, composed of the interior and the surface trapped zone, interacts with the wind to self-propel.

We have not included all possible large scale mixed layer processes in the present problem. Most importantly, we have neglected surface heat exchange, both in order to pose a problem that we had the resources to solve, and because it seemed as if we had little to learn from its inclusion. The resulting computations correspond to fall or winter Gulf Stream Rings. It seems from Chapter IV that the inclusion of air-sea exchange would simply modulate the intensity of the vertical flow. During the summer, we would expect the pumping to be weakened by the degradation of the SST anomaly; with the reemergence of the anomaly in the fall, southward motion should increase. From this standpoint, we really have nothing new to learn by including the surface buoyancy flux in the problem, and for this reason it was neglected. Still it is possible that the seasonality of the forcing could produce some novel results and it would be interesting to include a more active mixed layer in the calculation as a test of its importance.

The equations which were solved in this chapter assume that the mixed layer remains relatively shallow, in which case the alteration of the surface pressure field from that of the interior is negligible. Recall from Chapter II that the pressure field in the mixed layer begins to assume an independent character from that of the interior if either the temperature difference across the Ring becomes large, or if the layer becomes deep. During various periods of the year, the surface 
layers of warm core Rings meet both of these criteria, therefore we should endeavor to relax the thin layer assumption. However, the problem we obtain almost necessitates a move away froin quasi-geostrophic theory. Perhaps the most blatant ageostrophic effect comes from those isotherms which are deep in Ring center, but which rise to the base of the mixed layer at Ring flank. Second, the strong annual diabatic influence produces large changes in upper and intermediate layer buoyancy. Under such circumstances, buoyancy is no longer solely governed by vertical advection of a mean, time-independent density structure, i.e.:

$$
b_{t} \neq-\mathrm{wN}^{2} \text {. }
$$

Nor does this problem easily lend itself to numerical solution. To compute the response of a mixed layer requires fine vertical resolution; when investigating two horizontal dimensions as well, the computational requirements will be immense. Still, this is an important problem; we should see vertical circulations develop as the fluid adjusts to the density structure. If this is so, we need to understand how such a circulation affects Ring evolution and to assess its importance.

It is clear that there are other processes in need of investigation, and it is the hope of the author to continue working on all of the mentioned areas. It is equally clear, however, that the SST field of a Ring will affect the stress pattern in a manner similiar to that modeled here. The interesting result of this chapter is the self-propellant nature of the Ring-SST system caused by the temperature dependent coefficient of drag. Further experimentation will prove enlightening with respect to relative importance of this mechanism. 
CHAPTER VII. SUMMARY

In the present thesis, we have investigated several problems dealing with the evolution of both warm and cold core Rings. The importance of Rings to oceanic property distributions and the large scale circulation is currently unknown, although many estlmates suggest their effects are large. The primary motivation for the present work comes from a desire to better quantify their influence and, this being a difficult problem, we have made some small progress in this direction.

We have attempted to make some definitive statements with. regards to the processes which cause Rings to behave as they do. This thesis demonstrates that Ring-atmosphere interactions are an important component of Ring behavior, affecting their sea surface temperature field and their dynamic evolution. For example, we have demonstrated that spindown induced by frictional loss to the wind is strong enough to account for something between $30-100 \%$ of observed Ring spindown. Other mechanisms of decay which have been proposed include lateral dissipation, bottom drag, and wave drag, and with the exception of the latter, (Huang, personal communication) none have been demonstrated to have as large an effect as wind forcing. In addition, wind forcing can be computed in terms of known quantities. We have also shown that Ring SST cycles may be accounted for in terms of air-sea heat exchange, which differs from the pervading feeling that Ring dynamics are responsible for those cycles. Finally, we have shown that a significant fraction of the observed southward motion of Rings can be ascribed to the 
interaction of wind with Ring SST. The size of the induced propagation compares with that due to other suggested mechanisms and observed pattern propagation rates.

We have tested each of the processes individually, yet they affect different aspects of Ring evolution (propagation, decays, shallow temperature field) which are probably independent at first order. We can however combine the principal results to get an more global Ring picture. The study of particle trajectories in Rings strongly supports the aptness of the one-dimensional mixed layer models for the Ring trapped zone. The SST results from Chapter IV also indicate that the surface buoyancy equation used in Chapter VI, in which air-sea heat flux was neglected, is a reasonable model of wintertime Ring SST, and that warm Rings should be more affected by the propagation mechanism than cold. The calculations of Chapter III indicated how the SST configuration ought to be set up, and the information about the Lagrangian field of a Ring proved useful in the final dynamical understanding of the effects of the atmospheric forcing.

On the basis of the calculations, we can make some predictions with regards to oceanic observations. We have suggested that both warm and cold Rings are spun down by the wind. In Chapter IV, we performed a simple calculation which suggested that warm Rings are weakened significantly through heat loss to the atmosphere. Conversely, mixed layer response in cold Rings is confined to the near surface, so this energy loss mechanism is not in operation for them. We then can predict 
that warm Rings should decay faster than cold Rings, owing to the combination of mechanical and thermal energy loss. With respect to the questions about how significant Ring effects are on their surroundings, we can make a few qualitative statements. According to the Lagrangian analysis of Chapter III, the trapped zone of a Gulf Stream Ring corresponds to a pool of anomalous potential vorticity, $q$, and for particles to exit, their $q$ must change. The methods by which this can occur are limited to non-conservative processes, which unfortunately we are presently unable to model in a satisfactory fashion. Using crude biharmonic parametrizations, which yield roughly the observed decay rates, $q$ alteration is only strong enough to allow for a limited particle exchange at the trapped zone boundary. If we investigate Rings which decay by wind forcing, the results are similar to those in the biharmonic experiments. According to advection-diffusion experiments, fluxes in and out of the Ring are largely controlled by the size of the diffusion coeficients. In summary, tracer transport, from Ring core to exterior, is apparently controlled by the non-conservative and diffusive process about which we know little. It is generally felt, however that these processes are weak, especially in comparison to the estimates of the potential effect of Ring advection fields (Flierl and Dewar, 1981), so that Rings have probably been overestimated in their importance to Slope Water-Sargasso exchange (the Ring Group, 1981). Because the quantities depend on the coefficlents, it is difficult to say with confidence what their values are; however, in the wind-forced experiments of Chapter $v$, the vertical velocities in the upwelling region of a warm $R$ ing are consistent with a loss of 10-20\% of the 
trapped zone volume over a period of a year. Thus, published estimates which assume total Ring loss of material might be off by a factor of 5-10. While of a lesser magnitude, this still leaves Rings as a non-negligible material source for the Slope Water-Sargasso region. Also note that the faster decay of warm Rings is consistent with a greater trapped zone-to-Slope Water flux, so perhaps Ring flux is more important to the Slope.

With respect to Ring effects on exterior fluid, it has been postulated that Rings warp existing gradients, thus enhancing flux. For Rings travelling west, the advection-diffusion experiments indicate that the length of time during which individual particles interact with any Ring is short enough that north-south fluxes are only slightly enhanced. The accumulated effects of several Ring passages can be significant however (Flierl and Dewar, 1981). East-west fluxes, affected by the larger zonal displacements of particles by Rings have not really been tested here. Also, recall that we have demonstrated that north-south excursions of particles can be sizeable during Ring passage, and thus the presence of fronts, such as occur in the Slope Water, can enlarge meridional property flux.

\section{Suggestions-}

The formation of deep wintertime mixed layers most likely is a strong influence for warm core Rings, and in this thesis, we have not considered its dynamical implications. What we have learned is that the layers are formed by local air-sea exchange and anomalous deep buoyancy 
structure. We calculated that a warm Ring will be strongly spun down during wintertime (loss of 20 dynamic centimeters), so a careful modeling of the effects of buoyancy forcing on Rings is important to the estimates of Ring-induced flux to the Slope. Because of the dense grid required to accurately computed mixed layer response, the computation necessary to perform a combined mixed layer-quasigeostrophic interior calculation, with two horizontal dimensions, is very large, and will be costly as well as difficult to analyze. Therefore, it is the author's plan to commence on the above problem using a quasi-two dimensional (horizontal-vertical) model. Such a model neglects beta, but will probably prove informative in terms of Ring response.

From the Slope water XBT data, we note the development of temperature inversions in the upper 50 meters, from which we infer a sizeable vertical salt gradient. Second, we note that heat is getting in and out of the deep $(0(100 \mathrm{~m}))$ levels and that the temperature never gets well-mixed to these depths. Therefore, whatever is causing the mixing is different in different parts of the column. Hydrographic data from cold core Rings frequently shows similar, compensating, temperature-salt structure at the base of the mixed layer. It is not currently known how to model these mixed layer salt and temperature traces, or parameterize their effect on mixed layer density, but with respect to predicting sea surface temperature, it is important to understand how the incoming heat is distributed with depth. The data suggests that it is not a linear distribution, and that the presence of 
salt is having some influence. Whether double diffusive instabilities are responsible or can maintain the variations in turbulence is not clear. 


\section{REFERENCES}

Adamec, E., Elsberry, R., Garwood, R., and Haney, R.; 1981, An Imbedded Mixed Layer-Ocean Circulation Model, Dynamics of Atmospheres and Oceans, 6, 69-96.

Behringer, D.; Regier, L.; and Stommel, H.; 1979, Thermal Feedback on Wind Stress as a Contributing Cause of the Gulf Stream, Journal of Marine Research 37, 4, 699-709.

Bell, T. H.; 1978, Radiation Damping of Inertial Oscillations in the Upper Ocean, Journal of Fluid Mechanics, 88, 289-308.

Bunker, A. F.; 1976, Computations of Surface Energy Flux and Annual Air-Sea Interaction Cycles of the North Atlantic Ocean, Monthly Weather Review, 104, 1122-1140.

Cheney, R. and Richardson, P.; 1976, Observed Decay of a Cyclonic Gulf Stream Ring, Deep Sea Research, 23, 143-156.

Cheney, R. and Rfchardson, P.; 1977, Trajectory of a Satellite Tracked Buoy in a Kuroshio Cold Ring, Polymode News, 21 .

Colton, J. B.; and Stoddard, R. R.; 1972, Average Monthly Sea Water Temperatures: Nova Scotia to Long Island, 1940-1959, Serial Atlas of the Marine Environment, American Geographical Society.

Csanady, G. T.; 1973, Turbulent Diffusion in the Environment, D. ReideI Publishing Co., Vol.3, Geophysics and Astrophysics Monographs, Boston, 248 pp.

Deardorff, J. W.; 1968, Dependence of Air-Sea Transfer Coefficients on Bulk Stability, Journal of Geophysical Research, c3, 2549-2557.

Finlayson, B. A.; 1972, The Method of Weighted Residuals, Academic Press, New York N.Y.

Flierl, G.; 1977, The Application of Linear Quasi-Geostrophic Dynamics to Gulf Stream Rings, Journal of Physical Oceanography, 7 , 365-379.

Flierl, G.; 1978, Models of Vertical Structure and the Calibration of Two Layer Models, Dynamics of Atmospheres and Oceans, 2, 341-381.

Flierl, G.; 1979, Baroclinic Solitary Waves with Radial Symmetry, Dynamics of Atmospheres and Oceans, 3, 15-38.

Flierl, G. R.; 1981, Particle Motions in Large Amplitude Wave Fields, Geophysical and Astrophysical Fluid Dynamics, 18, 39-14.

Flierl, G. R.; 1982, A Model of the Structure and Motion of a Warm Core Ring, in preparation. 
Flierl, G. R. ; and Dewar, W. K.; 1981, Motion and Dispersal of Dumped Material By Large Amplitude Eddies, Wastes in the Ocean, Vol. 5, Wiley Interscience, Eds. D. Kester, P. Park, B. Ketchum, I. Duesdall.

Flierl, G. R.; Larichev, V. D.; McWilliams, J. C.; and Reznik, G. M.; 1980, The Dynamics of Baroclinic and Barotrophic Solitary Eddies, Dynamics of Atmospheres and Oceans, 5, 1-14.

Fuglister, F. C.; 1947, Average Monthly Sea Surface Temperatures of the Western North Atlantic, Papers in Physical Oceanography and Meteorology, 10, 1-25.

Fuglister, F. C.; 1972, Cyclonic Rings Formed by the Gulf Stream 1965-1966, Studies in Physical Oceanography, Vo1. 1, Gordon and Breach, 137-167.

Fuglister, F. and Worthington, R.; 1951, Some Results of a Multiple Ship Survey of the Gulf Stream, Tellus, $3,1-14$.

Gargett, A.; Sanford, T.; and Osborn, T.; 1979, Surface Mixing Layers in the Sargasso Sea, Journal of Physical Oceanography, 9, 1090-1111.

Gill A. E.; and Turner, J. S.; 1976, A Comparison of Seasonal Thermocline Models with Observation, Deep Sea Research, 23, $391-401$.

Gottlieb, D.; and Orszag S.; 1977, Numerical Analysis of Spectral Methods, Theory and Applications, SIAM, Boston.

Huynh, Q.; and Veronis, G.; 1981, The Effect of Temperature Dependent Coefficients on Poleward Heat Flux by Oceanic Gyres, Dynamics of Atmospheres and Oceans, 6, 49-66.

Ikeda, M.; 1982, Meanders and Detached Eddies of a Quasi-Geostrophic Model, Journal of Physical Oceanography, 4, 526-540.

Kantha, L. H.; 1977, Note on the Role of Internal Waves in the Thermocline Erosion, Modelling and Prediction of the Upper Layers of the Ocean, E. B. Kraus, Ed., Pergamon Press, 325 pp.

Klein, P.; and Coant1c, M.; 1981, A Numerical Study of Turbulent Processes in the Marine Upper Layers, Journal of Physical Oceanography, $11,6,849-863$.

Krauss, W.; 1981, The Erosion of a Thermocline, Journal of Physical Oceanography, $11,4,415-433$.

Leetma, A.; and Bunker, A.; 1978, Updated Charts of Mean Annual Wind Stress, Convergences of the Ekman Layer, and Sverdrup Transports in the North Atlantic, Journal of Marine Research, 36, 311-322.

Lai, David Y.; and Richardson, P. L.; 1977, The Distribution and Movement of Gulf Stream Rings, Journal of Physical Oceanography, 7 ,

Liu, Hsien-Ta; and Thompson R. O. R. Y.; 1976, Conversion of Eulerian to Lagrangian Statistics in One Dimension, 10, 109-128. 
McWilliams, J. C.; and Flierl, G. R.; 1979, On the Evolution of Isolated Nonlinear Vortices, Journal of Physical Oceanography, 9, $6,1155-1182$.

Mellor, G. L.; and Durbin, P. A.; 1975, The Structure and Dynamics of the Ocean Surface Mixed Layer, Journal of Physical Oceanography, 5, $718-728$

Mied, R.; and Lindemann, G.; 1979, The Propagation and Evolution of Cyclonic Gulf Stream Rings, Journal of Physical Oceanography, 9 , $1183-1206$.

Muller, P.; 1980, The Dynamics of Synoptic Scale Motions in the Ocean, Habilitationschrift Fachbereich Geowissenschaften Universitat Hamburg, 105pp., unpublished manuscript.

Needler G. T.; and Heath, R. A.; 1975, Diffusion Coefficients Calculated From the Mediterranean Salt Anomaly in the North Atlantic Ocean, Journal of Physical Oceanography, 5, 173-182.

Niller, P.; and Kraus, E. B.; 1977, One-Dimensional Models of the Upper Ocean, Modelling and Prediction of the Upper Layers of the Ocean, E. B. Kraus, Ed., Pergamon Press, 325 pp.

Nof, D.; 1982, On the $\beta$-Induced Movement of Isolated Baroclinic Eddies, Journal of Physical Oceanography, 12, 1662-1672.

01son, D.B.; 1980, The Physical Oceanography of Two Rings Observed by the Cyclonic Ring Experiment. Part II: Dynamics, Journal of Physical Oceanography, 10, 514-528.

Parker C. E.; 1971, Gulf Stream Rings in the Sargasso Sea, Deep Sea Research 18, 98 I-993.

Pedlosky, J.; 1979, Geophysical Fluid Dynamics, Springer-Verlag, 629 pp.

Pollard, R. T.; 1970, On the Generation by Winds of Inertial Waves in the Ocean, Deep Sea Research, 17, 795-812.

Pollard, R. T.; Rhines, P.B.; and Thompson, R.; 1973, The Deepening of the Wind Mixed Layer, Geophysical Fluid Dynamics, 3, 381-404.

Price, J; 1981, Upper Ocean Response to a Hurricane, Journal of Physical Oceanography, 11, 4, 415-433.

Price, J.; Mooers, C. N. K.; and Van Leer, J. L.; 1978, Observations and Simulation of Storm Induced Mixed Layer Deepening, Journal of Physical Oceanography, 8, 582-599.

Richardson, P. L.; 1980, Gulf Stream Ring Trajectories, Journal of Physical Oceanography, 10, 1, 90-104.

Richardson, P. L.; Cheney, R. E.; and Worthington, L. V.; 1978, A Census of Gulf Stream Rings, Spring 1975, Journal of Geophysical Research, 83, C12. 
Richardson, P. L.; Maillard, C.; and Sanford, T. B.; 1979, The Physical Structure and Life History of Cyclonic Gulf Stream Ring Allen, Journal of Geophysical Research, 84, C12.

Ring Group, the; 1981, Gulf Stream Cold Core Rings: Their Physics, Chemistry, and Biology, Science, 212, 4499.

Roache, P. J.; 1976, Computational Fluid Dynamics, Hermosa Publishers, Albuquerque, 446 PP.

Schmitz, J.; and Vastano, A.; 1975, Entrainment and Diffusion in a Gulf Stream Cyclonic Ring, Journal of Physical Oceanography, 5, 93-97.

Simpson J.J.; and Dickey, T. D.; 1981, Alternative Parameterizations of Downward Irradiance and Their Dynamical Significance, Journal of Physical Oceanography, $11,6,876-882$.

Stevenson, J. W.; 1979, On the Effect of Dissipation on Seasonal Thermocline Models, Journal of Physical Oceanography, 9, 1, 578-64.

Stevenson, J. W.; 1980, Response of the Surface Mixed Layer to QuasiGeostrophic Oceanic Motions, Harvard Ph.D. Thesis, 223 pp.

Thompson, R. O. R. Y.; 1973, Generation of Stochastic Processes with Given Spectrum, Utilitas Mathematica, 3, 127-137.

Thompson, R. O. R. Y.; 1976, Climatological Numerical Models of the Surface Mixed Layers of the Ocean, Journal of Physical Oceanography, 6, 496-502.

Thompson, R. O. R. Y.; 1982, A Potential-Flow Model of Turbulence Caused by Breaking Surface Waves, Journal of Geophysical Research, 87, C3, 1935-1938.

Vastano, A.; Schmitz, J.; and Hagan, D.; 198.0, The Physical Oceanography of Two Rings Observed by the Cyclonic Ring Experiment, Part I: Physical Structures, Journal of Physical Oceanography, 10, 4, 493-513.

Weibe, P. and Boyd, S.; 1978, Limits of 'Nematoscelis Megalops' in the Northwestern Atlantic in Relation to Gulf Stream Cold-Core Rings, Part I. Horizontal and Vertical Distributions, Journal of Marine Research, 36, 119.

Young, W. R.; 1981, The Vertical Structure of the Wind Driven Circulation, M.I.T.-W.H.O.I. Ph.D. Thesis, W.H.O.I. Document Number 81-89, 216pp. 


\section{ACXNOWLEDGEMENTS}

It has been my good fortune, during the past few years, to have labored on my thesis under the thoughtful tutelage of Dr. Glenn R. Flierl. And now $i t$ is my pleasure, and unique privilege, to unequivocally recommend him to up-and-coming doctoral students as an advisor of impeccable academic probity, and aptitude, and possessing the vision essential to the steerage of a thesis. I am honored to count him and his wife, Norma Kroll, amongst my friends. In addition, I have enjoyed the attentions of a most stimulating and thorough committee, composed of Drs. Dale Haidvogel, Philip Richardson, and Carl Wunsch, all of whom gave an early version of this manuscript a careful reading and offered an abundance of muchly appreciated criticisms. Special thanks go to Dr. Haidvogel, from whom I have learned computational methods, and to $\mathrm{Dr}$. Terry Joyce for chairing my thesis defense.

I would certainly be remiss were I not to mention the many valuable comments I have recieved from and discussions $I$ have had with Drs. William Schmitz, Paola Rizzoli, and Mark Cane, and I gratefully acknowledge Drs. Harry Bryden, Ed Harrison, and Mel Briscoe for their sage advice at critical points during my graduate student tenure.

This thesis has benefited from two summers' worth of my unofficial participation in the Geophysical Fluid Dynamics summer school, at which times I met and worked with Drs. Rory Thompson and Don Olson.

The Joint Program in Oceanography is endowed with as fine a collection of people as I suspect I shall ever meet. Two fellow students, Stephen Meacham and Rui X. Huang, have had more to do with the content of this thesis than I think elther of them realize. I have also profited handsomely from discussions with and the friendship of Karen Beggs, M. Benno Blumenthal, Al Campbell, Affonso Mascarenhas, Randy Patton, and Sophie Wacogne. I have been inspired by my companions and contemporaries Drs. William R. Young, Lawrence Pratt, and Lynne Talley, and have been so lucky as to share the final throes of completion with Dr. Teresa Chereskin.

Several of the figures in this thesis have been expertly drafted by Isabelle Kole, who patiently deciphered many a cryptic set of instructions written in my nearly illegible scrawl. A number of the sections in the final draft have been cast into shape by the efforts of Virginia I. Mills.

The Jolnt Program is most ably administrated by A. Lawrence Pierson and Mary Athanis, and the Department of Meteorology and Physical Oceanography by Jane $\mathrm{McNabb}$ and the aforementioned Miss Mills, all of whose efforts, I must say, have made my transit through MIT both pleasant and remarkably free of non-academic challenge.

Finally I thank my wife, Melinda, and my parents, Dr. and Mrs. William D. Dewar, who have patiently listened to, supported, and endured William K. Dewar, the graduate student, for five years, one month, and 24 days, a job which has been, shall we say, not without its trying moments.

The present research has been conducted under NOAA contract \# NA80AA-D-0057 and NSF contract \# OCE-8240455. The computational costs have been generously borne by Dr. Erik. Mollo-Christenson and ONR grant \# ONR $-c$ N00014-79-C-0838, and the maintenance and coherent operation of an inveterately disagreeable computer must be credited to the able and dogged persistence of Ken Morey. 